\title{
A tale of two groups: arithmetic groups and mapping class groups
}

\author{
Lizhen $\mathrm{Ji}^{*}$ \\ Department of Mathematics \\ University of Michigan \\ Ann Arbor, MI 48109 \\ (December 8, 2010)
}

\begin{abstract}
Summary
In this chapter, we discuss similarities, differences and interaction between two natural and important classes of groups: arithmetic subgroups $\Gamma$ of Lie groups $G$ and mapping class groups $\operatorname{Mod}_{g, n}$ of surfaces of genus $g$ with $n$ punctures. We also mention similar properties and problems for related groups such as outer automorphism groups $\operatorname{Out}\left(F_{n}\right)$, Coxeter groups and hyperbolic groups. Since groups are often effectively studied by suitable spaces on which they act, we also discuss related properties of actions of arithmetic groups on symmetric spaces and actions of mapping class groups on Teichmüller spaces, hoping to get across the point that it is the existence of actions on good spaces that makes the groups interesting and special, and it is also the presence of large group actions that also makes the spaces interesting. Interaction between locally symmetric spaces and moduli spaces of Riemann surfaces through the example of the Jacobian map will also be discussed in the last part of this chapter. Since reduction theory, i.e., finding good fundamental domains for proper actions of discrete groups, is crucial to transformation group theory, i.e., to understand the algebraic structures of groups, properties of group actions and geometry, topology and compactifications of the quotient spaces, we discuss many different approaches to reduction theory of arithmetic groups acting on symmetric spaces. These results for arithmetic groups motivate some results on fundamental domains for the action of mapping class groups on Teichmüller spaces. For example, the Minkowski reduction theory of quadratic forms is generalized to the action of $\operatorname{Mod}_{g}=\operatorname{Mod}_{g, 0}$ on the Teichmüller space $\mathcal{T}_{g}$ to construct an intrinsic fundamental domain consisting of finitely many cells,
\end{abstract}

\footnotetext{
*Partially Supported by NSF grant DMS 0905283
} 
solving a weaker version of a folklore conjecture in the theory of Teichmüller spaces.

On several aspects, more results are known for arithmetic groups, and we hope that discussion of results for arithmetic groups will suggest corresponding results for mapping class groups and hence increase interactions between the two classes of groups. In fact, in writing this survey and following the philosophy of this chapter, we noticed the natural procedure in $\S 5.12$ of constructing the Deligne-Mumford compactification of the moduli space of Riemann surfaces from the Bers compactification of the Teichmüller space by applying the general procedure of Satake compactifications of locally symmetric spaces, i.e., how to pass from a compactification of a symmetric space to a compactification of a locally symmetric space by making use of the reduction theory for arithmetic groups.

The layout of this chapter is as follows. In $\S 1$, the introduction, we discuss some general questions about discrete groups, group actions and transformation group theories. In $\S 2$, we summarize results on arithmetic subgroups $\Gamma$ of semisimple Lie groups $G$ and mapping class $\operatorname{groups} \operatorname{Mod}_{g, n}$ of surfaces of genus $g$ with $n$ punctures, and their actions on symmetric spaces of noncompact type and Teichmüller spaces respectively. ${ }^{1}$ For comparison and for the sake of completeness, we also discuss corresponding properties of three related classes of groups: outer automorphism groups of free groups, Coxeter groups and hyperbolic groups. In $\S 3$, we describe several sources where discrete groups and discrete transformation groups arise. In $\S 4$ and $\S 5$ we give definitions and details of some of the properties listed earlier in $\S 2$ for arithmetic groups and mapping class groups. In the last section, $\S 6$, we deal with the coarse Schottky problem, a large scale geometric generalization of the classical Schottky problem of characterizing the Jacobian varieties among abelian varieties, i.e., the image of the Jacobian map from the moduli space $\mathcal{M}_{g}$ of compact Riemann surfaces of genus $g$ to the Siegel modular variety $\mathcal{A}_{g}$, an important Hermitian locally symmetric space, which is equal to the moduli space of principally polarized abelian varieties of dimension $g$.

For the detailed organization of this chapter, see the table of contents starting on the next page.

2000 Mathematics Subject Classification: 53C35, 30F60, 22E40, 20G15, 57M99.

Keywords: Arithmetic groups, mapping class groups, symmetric spaces, Teichmüller spaces, Lie groups, transformation groups, proper actions, classifying spaces, locally symmetric spaces, moduli spaces, Riemann surfaces, reduction theories, fundamental domains, compactifications, boundaries, universal spaces, duality groups, curve complexes, Tits buildings, hyperbolic groups, Coxeter groups, outer automorphism groups, Schottky problems, pants decompositions.

\footnotetext{
${ }^{1}$ The lists are certainly not complete and only results known to us are listed.
} 


\section{Contents}

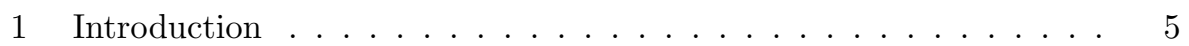

1.1 Summary ....................... 5

1.2 General questions on discrete groups and discrete transformation groups . . . . . . . . . . . . . . 6

2 Summaries of properties of groups and spaces in this chapter . . . 13

2.1 Properties of arithmetic groups $\Gamma \ldots \ldots \ldots \ldots$

2.2 Properties of actions of arithmetic groups $\Gamma$ on symmetric spaces

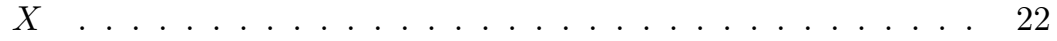

2.3 Properties of mapping class groups $\operatorname{Mod}_{g, n} \ldots \ldots \ldots \ldots 27$

2.4 Properties of actions of mapping class $\operatorname{groups} \operatorname{Mod}_{g, n}$ on Teichmüller spaces . . . . . . . . . . . . . . . 31

2.5 Properties of outer automorphism groups $\operatorname{Out}\left(F_{n}\right) \ldots \ldots$

2.6 Properties of the outer space $X_{n}$ and the action of $\operatorname{Out}\left(F_{n}\right)$ on

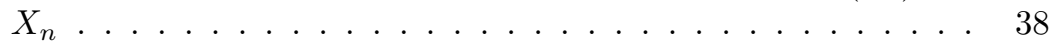

2.7 Properties of Coxeter groups . . . . . . . . . . . . . 40

2.8 Properties of hyperbolic groups . . . . . . . . . . . . 41

3 How discrete groups and proper transformation groups arise . . . . 44

3.1 Finitely generated groups, Cayley graphs and Rips complexes . 44

3.2 Rational numbers and $p$-adic norms . . . . . . . . . . . 46

3.3 Discrete subgroups of topological groups . . . . . . . . . . 46

3.4 Fundamental groups and universal covering spaces . . . . . . 47

3.5 Moduli spaces and Mapping class groups . . . . . . . . . . . 47

3.6 Outer automorphism groups . . . . . . . . . . . . . . . 48

3.7 Combinatorial group theory . . . . . . . . . . . . . . . . 49

3.8 Symmetries of spaces and structures on these spaces . . . . . 49

4 Arithmetic groups . . . . . . . . . . . . . . . . . 50

4.1 Definitions and examples . . . . . . . . . . . . 50

4.2 Generalizations of arithmetic groups: non-arithmetic lattices . 55

4.3 Generalizations of arithmetic groups: $S$-arithmetic subgroups . 56

4.4 Generalizations of arithmetic groups: Non-lattice discrete subgroups and Patterson-Sullivan theory . . . . . . . . . . 58

4.5 Symmetric spaces and actions of arithmetic groups . . . . . . 59

4.6 Fundamental domains and generalizations . . . . . . . . . 62

4.7 Fundamental domains for Fuchsian groups and applications to compactification . . . . . . . . . . . 66

4.8 Minkowski reduction theory for $\operatorname{SL}(n, \mathbb{Z}) \ldots \ldots \ldots \ldots$

4.9 Reduction theory for general arithmetic groups . . . . . . . 74

4.10 Precise reduction theory for arithmetic groups . . . . . . . . . 77

4.11 Combinatorial properties of arithmetic groups: finite presentation and bounded generation . . . . . . . . . . 83

4.12 Subgroups and overgroups . . . . . . . . . . . . . . . 84 
4.13 Borel density theorem . . . . . . . . . . . . . . . 86

4.14 The Tits alternative and exponential growth . . . . . . . . 87

4.15 Ends of groups and locally symmetric spaces . . . . . . . . 88

4.16 Compactifications and boundaries of symmetric spaces . . . . 89

4.17 Baily-Borel compactification of locally symmetric spaces . . . . 93

4.18 Borel-Serre compactification of locally symmetric spaces and cohomological properties of arithmetic groups . . . . . . 9 95

4.19 The universal spaces $E \Gamma$ and $\underline{E} \Gamma$ via the Borel-Serre partial compactification . . . . . . . . . . . . 97

$5 \quad$ Mapping class groups $\operatorname{Mod}_{g, n} \ldots \ldots \ldots \ldots \ldots$

5.1 Definitions and examples . . . . . . . . . . . . . . . . . . . 99

5.2 Teichmüller spaces . . . . . . . . . . . . . . . . . . . . 100

5.3 Properties of Teichmüller spaces _ . . . . . . . . . . . . . 103

5.4 Metrics on Teichmüller spaces . . . . . . . . . . . . . . . . . 103

5.5 Compacfications and boundaries of Teichmüller spaces . . . . . 104

5.6 Curve complexes and boundaries of partial compactifications . 106

5.7 Universal spaces for proper actions . . . . . . . . . . . . . 108

5.8 Cohomological properties of $\operatorname{Mod}_{g, n} \ldots \ldots \ldots$

5.9 Pants decompositions and Bers constant . . . . . . . . . . 110

5.10 Fundamental domains and rough fundamental domains . . . . . 111

5.11 Generalized Minkowski reduction and fundamental domains . . 113

5.12 Compactifications of moduli spaces and a conjecture of Bers . . 118

5.13 Geometric analysis on moduli spaces . . . . . . . . . . . . . 124

$6 \quad$ Interactions between locally symmetric spaces and moduli spaces of Riemann surfaces . . . . . . . . . . . . . . . . 125

6.1 The Jacobian map and the Schottky problem . . . . . . . . 126

6.2 The coarse Schottky problem . . . . . . . . . . . . . 128 


\section{Introduction}

\subsection{Summary}

In this chapter, we discuss similarities, differences and interaction between two natural and important classes of groups: arithmetic subgroups $\Gamma$ of Lie groups $G$ and mapping class groups $\operatorname{Mod}_{g, n}$ of surfaces of genus $g$ with $n$ punctures. We also mention similar properties and problems for related groups such as outer automorphism groups $\operatorname{Out}\left(F_{n}\right)$, Coxeter groups and hyperbolic groups. Since groups are often effectively studied by suitable spaces on which they act, we also discuss related properties of actions of arithmetic groups on symmetric spaces and actions of mapping class groups on Teichmüller spaces, hoping to get across the point that it is the existence of actions on good spaces that makes the groups interesting and special, and it is also the presence of large group actions that also makes the spaces interesting. Interaction between locally symmetric spaces and moduli spaces of Riemann surfaces through the example of the Jacobian map will also be discussed in the last part of this chapter. Since reduction theory, i.e., finding good fundamental domains for proper actions of discrete groups, is crucial to transformation group theory, i.e., to understand the algebraic structures of groups, properties of group actions and geometry, topology and compactifications of the quotient spaces, we discuss many different approaches to reduction theory of arithmetic groups acting on symmetric spaces. These results for arithmetic groups motivate some results on fundamental domains for the action of mapping class groups on Teichmüller spaces. For example, the Minkowski reduction theory of quadratic forms is generalized to the action of $\operatorname{Mod}_{g}=\operatorname{Mod}_{g, 0}$ on the Teichmüller space $\mathcal{T}_{g}$ to construct an intrinsic fundamental domain consisting of finitely many cells, solving a weaker version of a folklore conjecture in the theory of Teichmüller spaces.

On several aspects, more results are known for arithmetic groups, and we hope that discussion of results for arithmetic groups will suggest corresponding results for mapping class groups and hence increase interactions between the two classes of groups. In fact, in writing this survey and following the philosophy of this chapter, we noticed the natural procedure in $\S 5.12$ of constructing the Deligne-Mumford compactification of the moduli space of Riemann surfaces from the Bers compactification of the Teichmüller space by applying the general procedure of Satake compactifications of locally symmetric spaces, i.e., how to pass from a compactification of a symmetric space to a compactification of a locally symmetric space by making use of the reduction theory for arithmetic groups.

The layout of this chapter is as follows. In the rest of this introduction, we discuss some general questions about discrete groups, group actions and transformation group theories. In $\S 2$, we summarize results on arithmetic 
subgroups $\Gamma$ of semisimple Lie groups $G$ and mapping class groups $\operatorname{Mod}_{g, n}$ of surfaces of genus $g$ with $n$ punctures, and their actions on symmetric spaces of non-compact type and Teichmüller spaces respectively. ${ }^{2}$ For comparison and for the sake of completeness, we also discuss corresponding properties of three related classes of groups: outer automorphism groups of free groups, Coxeter groups and hyperbolic groups. In $\S 3$, we describe several sources where discrete groups and discrete transformation groups arise. In $\S 4$ and $\S 5$ we give definitions and details of some of the properties listed earlier in $\S 2$ for arithmetic groups and mapping class groups. In the last section, $\S 6$, we deal with the coarse Schottky problem, a large scale geometric generalization of the classical Schottky problem of characterizing the Jacobian varieties among abelian varieties, i.e., the image of the Jacobian map from the moduli space $\mathcal{M}_{g}$ of compact Riemann surfaces of genus $g$ to the Siegel modular variety $\mathcal{A}_{g}$, an important Hermitian locally symmetric space, which is equal to the moduli space of principally polarized abelian varieties of dimension $g$.

For the detailed organization of this chapter, see the table of contents that proceeds this introduction.

\subsection{General questions on discrete groups and discrete transformation groups}

Groups are fundamental objects and they describe symmetry in mathematics and sciences. Basically, there are two kinds of groups: (1) discrete groups, i.e., groups with the discrete topology, (2) non-discrete (or continuous) groups, in particular, Lie groups. These two classes of groups are closely related in many ways and embeddings of discrete groups into non-discrete groups give rise to interesting transformation groups, as seen in various results about the fundamental pair of groups $\mathbb{Z} \subset \mathbb{R}$. Of course, any group can be given the discrete topology and hence considered as a discrete group. On the other hand, as far as discrete groups are concerned, it is probably most natural and fruitful to study groups that occur naturally as discrete subgroups of topological groups or as discrete transformation groups, i.e., discrete groups acting properly discontinuously on topological spaces that have some reasonable properties, for example, being locally compact.

In this expository chapter, we discuss two important classes of infinite discrete groups: (1) arithmetic subgroups of linear algebraic groups such as $\mathrm{SL}(n, \mathbb{Z})$ and $\mathrm{GL}(n, \mathbb{Z})$ and discrete subgroups $\Gamma$ of Lie groups $G ;(2)$ mapping class groups $\operatorname{Mod}_{g, n}$ of compact orientable surfaces $S_{g, n}$ of genus $g$ with $n$ points removed (i.e., with $n$ punctures).

We will discuss similarities and differences between these two classes of groups and their properties. There have been several excellent surveys on

\footnotetext{
${ }^{2}$ The lists are certainly not complete and only results known to us are listed.
} 
properties of mapping class groups from different perspectives and comparison with arithmetic groups, for example, [124] [169] [188] [190] [317] [54] [164] [321]. We hope that the current survey is complementary to the existing ones.

We will also discuss similar properties of some related groups. For example, the family of outer automorphism groups $\operatorname{Out}\left(F_{n}\right)$ of free groups $F_{n}$ is closely related to the two classes of groups mentioned above, and we will also discuss briefly their properties. There are also excellent surveys on these topics such as [73] [344] [412]. Other closely related families of groups discussed briefly in this chapter include Coxeter groups and hyperbolic groups.

Since groups first arose as symmetries or transformation groups of number fields and differential equations, and since many properties of groups can be understood by studying their actions on suitable spaces, we will emphasize the point of view of geometric transformation groups. We will also study two classes of spaces naturally associated with the above two classes of groups: (1) symmetric spaces of semisimiple Lie groups and more general homogeneous spaces; (2) Teichmüller spaces of marked Riemann surfaces. Furthermore, we will study actions of these groups on such spaces and their quotients, which are locally symmetric spaces, and moduli spaces of Riemann surfaces (or algebraic curves) respectively. Besides being important for understanding properties of the groups, these spaces are also interesting in themselves. In some sense, the groups are studied in order to understand the spaces on which the groups act and also to understand the quotients of the actions. The groups themselves have sometimes played a secondary role in some applications. For example, Teichmüller spaces and actions of mapping class groups on them were originally studied in order to understand the moduli spaces of Riemann surfaces. It is often the case that a group action contains more information than the quotient, as seen, for example, in the context of equivariant cohomology theory.

Given a discrete group $\Gamma$, the following problems are natural:

(1) Finite generation of $\Gamma$ and some variants, for example, bounded generation. In general, it is much more difficult to find explicit generators than to prove existence.

(2) Finite presentation of $\Gamma$ and derivation or understanding other properties from the presentation.

(3) Internal structures such as finite subgroups, subgroups of finite index, normal subgroups of $\Gamma$, and overgroups of finite index (i.e., groups that contain $\Gamma$ as subgroups of finite index). Existence of torsion-free subgroups of finite index is important and allows one to define virtual properties of $\Gamma$.

(4) Combinatorial properties of $\Gamma$ such as the word problem, the conjugacy problem, and the isomorphism problem for classes of groups that contain $\Gamma$. 
(5) Other finiteness properties of $\Gamma$ such as $F P_{\infty}, F P, F L$ in homological algebra and the existence of CW-models of classifying (or universal) spaces $E \Gamma$ for proper and fixed point free actions of $\Gamma$ and $\underline{E} \Gamma$ for proper actions of $\Gamma$, which satisfy various finiteness conditions, for example, existence of only finitely many cells in all dimensions modulo $\Gamma$, of finitely many cells in each dimension modulo $\Gamma$, or $E \Gamma$ and $\underline{E} \Gamma$ being of finite dimension.

(6) Cohomological groups and cohomological invariants of $\Gamma$ such as cohomological dimension and the Euler characteristic, and properties such as the Poincaré duality and generalized Poincaré duality properties.

(7) Other algebraic invariants of $\Gamma$ and its associated group ring $\mathbb{Z} \Gamma$ such as $K$-groups and $L$-groups of the group ring $\mathbb{Z} \Gamma$ in the algebraic and geometric topology.

(8) Large scale properties of $\Gamma$ endowed with word metrics (or equivalently its Cayley graphs with each edge of length 1) such as growth rate, quasirigidity properties, asymptotic dimension, the rationality of the growth series, and large scale properties of infinite subgroups of $\Gamma$ such as bounds on the distortion function.

(9) External properties: existence of linear representations and their properties such as Property $\mathrm{T}$ for $\Gamma$-actions on Hilbert spaces, and existence of actions on topological spaces and manifolds (i.e., non-linear representations) and their properties such as Property FA of Serre for actions on trees.

(10) Realizations of $\Gamma$ as subgroups of linear groups, discrete subgroups of Lie groups and other topological groups.

(11) Ends, compactifications and boundaries of $\Gamma$ and related $\Gamma$-spaces when the group $\Gamma$ is infinite.

As pointed out earlier, a notion closely related to the one of discrete group is that of topological transformation group. Many of the above properties of groups $\Gamma$ can be studied and understood by using actions of $\Gamma$ on suitable topological spaces. On the other hand, finding the right space is often not easy, and general groups probably do not act on spaces with desirable properties since such actions usually impose some conditions on the groups. The groups studied in this chapter do act on good spaces in the sense that the spaces have rich structures that can be described and understood, and hence they are special and interesting from this point of view.

For discrete subgroups $\Gamma$ of Lie groups $G$, it is relatively easy to find spaces on which these groups $\Gamma$ act. For example, there are natural classes of homogeneous spaces associated with the Lie groups $G$ on which the discrete groups $\Gamma$ act. But for other discrete groups such as the mapping class groups of surfaces $\operatorname{Mod}_{g, n}$ and the outer automorphism groups of free groups $\operatorname{Out}\left(F_{n}\right)$, the situation is more complicated and the construction of analogous spaces is less 
direct. For the mapping class groups of general manifolds and for automorphism groups of general discrete groups, the construction of analogous spaces are not known and might not be possible. This makes $\operatorname{Mod}_{g, n}$ and $\operatorname{Out}\left(F_{n}\right)$ really special.

We also note that the idea of transformation groups was motivated and occurred before the concept of group was introduced. For example, translations and rotations in the plane $\mathbb{R}^{2}$, in the space $\mathbb{R}^{3}$ and their compositions were known in the ancient times, though not in the language of group theory. When the concept of group was formally introduced, it was also introduced for transformation groups on roots of algebraic equations.

Let $X$ be a topological space. Assume that a group $\Gamma$ acts on it by homeomorphisms. Then the pair $(\Gamma, X)$ is called a topological transformation group. We always assume that the action is proper, i.e., for any compact subset $K \subset X$, the subset $\{\gamma \in \Gamma \mid \gamma K \cap K \neq \emptyset\}$ is compact. When $\Gamma$ is given the discrete topology, this is equivalent to the fact that $\Gamma$ acts properly discontinuously on $X$, i.e., for any compact subset $K \subset X$, the subset $\{\gamma \in \Gamma \mid \gamma K \cap K \neq \emptyset\}$ is finite. In the literature and also in the following, if a discrete group acts properly discontinuously on a topological space, we often say that the group acts properly on the space. ${ }^{3}$

In the following, we assume that $\Gamma$ is a discrete group and that $\Gamma$ acts properly on $X$. Such a $\Gamma$-space $X$ is called a proper $\Gamma$-space, and the pair $(\Gamma, X)$ is called a proper transformation group, or a discrete transformation group.

For any proper $\Gamma$-space, the following questions are natural:

(1) The structure of each orbit of $\Gamma$ and its relation with the ambient space $X$. This is closely related to structures of finite subgroups of $\Gamma$. Suppose that $X$ is a metric space and $\Gamma$ acts isometrically. Then another natural question is whether $\Gamma$ endowed with the word metric is quasi-isometric to the $\Gamma$-orbits endowed with the induced subspace metric from $X$.

(2) The nature of fixed point sets $X^{F}$ in $X$ of finite subgroups $F$ of $\Gamma$. For example, is the fixed points set $X^{F}$ nonempty? If $X$ is contractible, is the fixed points set $X^{F}$ contractible?

(3) The structure of the quotient $\Gamma \backslash X$. For example, when is $\Gamma \backslash X$ compact? Suppose that $\Gamma \backslash X$ is noncompact. What is its end structure? How to

\footnotetext{
${ }^{3}$ Non-proper actions of infinite discrete groups occur naturally and also play an important role in understanding structures of the groups. For example, the action of $\mathrm{SL}(2, \mathbb{Z})$ on $\mathbf{H}^{2}$ extends continuously to the boundary $\mathbf{H}^{2}(\infty)=S^{1}$, and the extended action on the boundary $S^{1}$ is not proper. This is a special case of the action of an arithmetic subgroup $\Gamma \subset G$ on the Furstenberg boundaries of a semisimple Lie group $G$. This action on the Furstenberg boundaries has played a crucial role in understanding rigidity properties of arithmetic groups. Another type of problems related to understanding quotients of nonproper actions, or rather the non-proper actions themselves, occurs in non-commutative geometry.
} 
compactify it? Suppose that $X$ has a measure which is invariant under the left action of $\Gamma$. When does $\Gamma \backslash X$ have finite measure? Suppose that $X$ is a geodesic space. What is structure of geodesics of $\Gamma \backslash X$ that go to infinity? ${ }^{4}$ What are properties of the geodesic flow of $\Gamma \backslash X$ ? For example, is it ergodic? Another natural problem concerns existence and distribution of closed geodesics of $\Gamma \backslash X$, which correspond to periodic orbits of the geodesic flow.

(4) Suppose that $X$ is a Riemannian manifold. What are the spectral properties of the Laplace operator of $\Gamma \backslash X$ ? For example, one can ask about the existence of continuous spectrum and discrete spectrum (or $L^{2}$-eigenvalues) of $\Gamma \backslash X$, the existence of generalized eigenfunctions for the continuous spectrum, the existence of discrete spectrum inside the continuous spectrum, the asymptotic behavior of the counting function of the discrete spectrum, and the connection between the spectral theory and the geometry of $\Gamma \backslash X$, for example, the lengths of closed geodesics.

(5) Let $X$ be a Riemannian manifold and $\Gamma$ be an infinite discrete group. What are $L^{2}$-invariants of $X$ with respect to the action of $\Gamma$, and what are the relations with the corresponding invariants of $\Gamma \backslash X ?^{5}$

(6) Fundamental domains for the $\Gamma$-action on $X$ and rough fundamental domains for $\Gamma$ satisfying various finiteness conditions. How to construct them and how to understand their shapes at infinity if $\Gamma \backslash X$ is noncompact? How are these fundamental domains related to the structure at infinity of $X$ and $\Gamma \backslash X$ ?

(7) Assume that $\Gamma$ is infinite and hence $X$ is noncompact. What kind of compactifications does $X$ have? How does $\Gamma$ act on the boundaries of these compactifications? What are the properties of limit sets of orbits of $\Gamma$ on the boundaries and what are their relations to other properties of $\Gamma$ or to the quotient $\Gamma \backslash X$ ?

(8) Relations between structures of $X$ and $\Gamma \backslash X$ such as compactifications and function theory if $X$ is a Riemannian manifold. For example, how can compactifications of $X$ be used to compactify noncompact quotients $\Gamma \backslash X$ ? How are eigenfunctions and spectra of $X$ and $\Gamma \backslash X$ related?

In studying these problems, constructing good fundamental domains is a crucial step. The best example to explain this fact is the action of Fuchsian groups on the Poincaré upper half plane. Because of this consideration, in this chapter, we will discuss various aspects of fundamental domains and rough fundamental domains for actions of arithmetic groups on symmetric spaces in

\footnotetext{
${ }^{4} \mathrm{~A}$ striking application of understanding structures going to infinity is the McShane identity in [301, Corollary 5, Theorems 4 \& 2].

${ }^{5}$ The basic point of view is that instead of taking $\Gamma$-invariant functions or differential forms, one considers $L^{2}(X)$ as a representation of $\Gamma$ and takes the von Neumann dimension. See [270] for details.
} 
some detail. This is an important part of the foundational reduction theory of arithmetic groups. These results motivate results on fundamental domains for the action of $\operatorname{Mod}_{g, n}$ on the Teichmüller space $\mathcal{T}_{g, n}$.

If $X$ is a metric space and if $\Gamma$ acts cocompactly and isometrically on $X$, then $\Gamma$ with any word metric associated to a finite generating set is quasiisometric to $X$, and boundaries of $X$ can often naturally be thought of as boundaries of $\Gamma$. On the other hand, in many instances, the quotient of $\Gamma \backslash X$ is not compact, and boundaries of $X$ can also be considered as boundaries of $\Gamma$, and they have played a fundamental role in the study of $\Gamma$. This is the case for non-uniform arithmetic groups discussed below, especially in their rigidity properties.

As mentioned before, for general groups $\Gamma$, it is not easy to find suitable $\Gamma$ spaces. On the other hand, there are some general constructions of such spaces. A particularly important class of $\Gamma$-spaces is the class of universal spaces for proper actions of $\Gamma$. These spaces are unique up to homotopy equivalence and are usually denoted by $\underline{E} \Gamma$. They are the terminal spaces in the category of proper $\Gamma$-spaces.

An $\underline{E} \Gamma$-space is characterized by the following conditions: (a) $\Gamma$ acts properly on $\underline{E} \Gamma$. (b) For any finite subgroup $F \subset \Gamma$, the set of fixed points $(\underline{E} \Gamma)^{F}$ is nonempty and contractible. In particular, $\underline{E} \Gamma$ is contractible.

If $\Gamma$ is torsion-free, then $\underline{E} \Gamma$ is the universal space $E \Gamma$ for proper and fixed point free actions of $\Gamma$, which is also the universal covering space of a classifying space $B \Gamma$ of $\Gamma$, where $B \Gamma$ is characterized uniquely up to homotopy equivalence by the conditions: $\pi_{1}(B \Gamma)=\Gamma$, and $\pi_{i}(B \Gamma)=\{1\}$ for $i \geq 2$.

It is known that if $\Gamma$ is virtually torsion-free, i.e., if there exists a finite index torsion-free subgroup of $\Gamma$, then for any model $X$ of $\underline{E} \Gamma$, the virtual cohomological dimension vcd $\Gamma$ satisfies the upper bound:

$$
\operatorname{vcd} \Gamma \leq \operatorname{dim} X \text {. }
$$

It was proved by Serre [384] that if $\Gamma$ is virtually torsion-free and vcd $\Gamma$ is finite, then there exists a finite dimensional model of $\underline{E} \Gamma$. Some natural questions concerning $\underline{E} \Gamma$ are the following:

(1) Assume that $\Gamma$ is virtually torsion-free. Does there exist a model $X$ of $\underline{E} \Gamma$ such that $\operatorname{dim} X=\operatorname{vcd} \Gamma$ ?

(2) Assume that $\Gamma$ is virtually torsion-free and vcd $\Gamma$ is finite. Is there a model $X$ of $\underline{E} \Gamma$ such that $\Gamma \backslash X$ is compact? Furthermore, can $X$ satisfy the condition $\operatorname{dim} X=\operatorname{vcd} \Gamma$ ?

(3) Assume that $\Gamma$ is virtually torsion-free and $\operatorname{vcd} \Gamma$ is finite. Given a natural model $X$ of $\underline{E} \Gamma$ such that $\Gamma \backslash X$ is noncompact, is it possible to find a $\Gamma$-equivariant compactification $\bar{X}$ of $X$ such that the inclusion $X \hookrightarrow \bar{X}$ is a homotopy equivalence? Is it possible to find a $\Gamma$-stable subspace with a compact quotient (or rather a finite CW complex) under 
$\Gamma$ such that it is an equivariant deformation retraction of $X$ ? If $\operatorname{dim} X>$ vcd $\Gamma$, is there a $\Gamma$-equivariant deformation retract $S$ of $X$ such that $\operatorname{dim} S=\operatorname{vcd} \Gamma$ and $\Gamma \backslash S$ is compact? (Such deformation retracts $S$ are often called spines of $X$. More generally, any cocompact deformation retract is also called a spine of $X$.)

(4) Assume that $\Gamma$ is torsion-free and satisfies the Poincaré duality. When does $\Gamma$ admit a model of $B \Gamma$ given by a compact manifold without boundary? If such a manifold model of $B \Gamma$ exists, is it unique up to homeomorphism?

(5) For some important arithmetic groups such as $\operatorname{SL}(n, \mathbb{Z})$, find explicit models of $E \Gamma$ and $\underline{E} \Gamma$ given by $\mathrm{CW}$-complexes so that we can compute cohomology and homology groups of $\Gamma$.

Questions (2) and (3) are closely related to the problem of existence of fundamental domains for $\Gamma$ that are unions of simplices, or $\Gamma$-equivariant cell decompositions of $X$. In fact, once $X$ has a $\Gamma$-equivariant simplicial complex structure, then it is easier to construct deformation retracts and spines. The second part of question (4) is called the Borel conjecture for $\Gamma$, which asserts that two closed aspherical manifolds with the same fundamental group $\Gamma$ are homeomorphic to each other. (Note that if $M$ is an aspherical manifold, i.e., $\pi_{i}(M)=\{1\}$ for $i \geq 2$, then it is a model of $B \Gamma$ for $\Gamma=\pi_{1}(M)$.) See [132] [136] for precise statements and references.

Though arithmetic subgroups of semisimple Lie groups and mapping class groups are virtually torsion-free, some other natural groups, for example $S$ arithmetic subgroups of algebraic groups of positive rank over function fields such as $\operatorname{SL}\left(n, F_{p}[t]\right)$, where $F_{p}$ is a finite field and $t$ is a variable, are not virtually torsion-free. Then their cohomological dimensions are equal to infinity. For such groups, finding good models of the universal spaces for proper actions is still important.

In this chapter, we will discuss results addressing the above questions for arithmetic groups and mapping class groups. Many results are also known for related groups such as the outer automorphism group $\operatorname{Out}\left(F_{n}\right)$ of the free group $F_{n}$, Coxeter groups and hyperbolic groups.

Besides these common questions and properties shared by these different classes of groups and spaces, we will also discuss the Jacobian (or period) map between the moduli space $\mathcal{M}_{g}$ of compact Riemann surfaces of genus $g$ and the Siegel modular variety $\mathcal{A}_{g}$, which is an important arithmetic locally symmetric space and is also the moduli space of principally polarized abelian varieties of dimension $g$, to show that there are interactions between these different spaces.

We hope that the results presented in this chapter will justify the rather unusual title of this chapter: as in the famous book "A tale of two cities" by Charles Dickens, what happened in London, Paris and on ways between them made the whole story interesting and exciting. Arithmetic groups and mapping 
class groups are interesting in their own, but analogies and relations between them have motivated many new problems and results for each of these groups. The Jacobian map between $\mathcal{M}_{g}$ and $\mathcal{A}_{g}$ has also played an important role in understanding $\mathcal{M}_{g}$. Therefore, various results and perspectives on arithmetic groups, mapping class groups and their associated spaces are all different parts of one tale!

Acknowledgments.

I would like to thank A. Papadopoulos for the invitation to contribute to this volume of Handbook of Teichmüller theory, which motivated me to write this chapter with an unusual title, for reading this chapter very carefully several times and making many helpful suggestions, and for pointing out the references [82] [415]. I would also like to thank Lixin Liu for pointing out the conjecture of Bers [42, Conjecture IV], S. Prassidis for valuable information about Coexter groups, R. Spatzier for the references [240] and [329], and Weixu $\mathrm{Su}$ for pointing out the presence of some half Dehn twists in the stabilizer of ordered pants decompositions. A part of the writing of this chapter was carried out during my visit to MSC, Tshinghua University, 2010 and I would like thank them for providing a very good working environment. Finally I would like to thank two referees for reading carefully a preliminary version of this chapter and making many helpful suggestions.

\section{Summaries of properties of groups and spaces in this chapter}

To emphasize similarities and differences between arithmetic groups and mapping class groups on the one hand, and between symmetric spaces and Teichmüller spaces on the other hand, we make four lists of properties for them in parallel. The paper of Harer [169] is a valuable reference on comparing the properties of arithmetic groups and mapping class groups, mainly concentrating on cohomological properties. The surveys [188] and [124] are also extensive and cover many different topics. Besides studying arithmetic groups and mapping class groups, the surveys [75] [344] also compare the similarities between these groups and the outer automorphism groups of free groups. We try to include some other properties and hope to provide a different perspective. On the other hand, it is clear from the table of contents that the current survey is not comprehensive, and many results on arithmetic groups and mapping class groups are not mentioned. For more results about mapping class groups, see also the books [124] [334] [335]. For more references on arithmetic groups, see [198] [39] [411]. 
After these four lists, for comparison, we also list some similar properties of $\operatorname{Out}\left(F_{n}\right)$ and two other important classes of groups: Coxeter groups and hyperbolic groups. We will be less exhaustive about properties of these latter three classes of groups.

We state properties to be discussed in this chapter in the following order:

(1) arithmetic groups (§2.1),

(2) symmetric spaces and locally symmetric spaces ( $\$ 2.2)$,

(3) mapping class groups $\operatorname{Mod}_{g, n}(\S 2.3)$,

(4) Teichmüller spaces and moduli spaces $(\S 2.4)$,

(5) outer automorphism groups $\operatorname{Out}\left(F_{n}\right)(\S 2.5)$,

(6) outer spaces $X_{n}(\S 2.6)$;

(7) Coxeter groups $(\S 2.7)$,

(8) hyperbolic groups $(\S 2.8)$.

\subsection{Properties of arithmetic groups $\Gamma$}

The following notation will be used in this chapter. Let $\mathbf{G} \subset \mathrm{GL}(n, \mathbb{C})$ be a linear semisimple algebraic group defined over $\mathbb{Q}$, and $G=\mathbf{G}(\mathbb{R})$ the real locus of $\mathbf{G}$, a real Lie group with finitely many connected components. ${ }^{6}$

Let $K \subset G$ be a maximal compact subgroup. Then the homogeneous space $X=G / K$ with an invariant metric is a symmetric space of noncompact type, in particular it is a simply connected and nonpositively curved complete Riemannian manifold. Let $\Gamma \subset \mathbf{G}(\mathbb{Q})$ be an arithmetic subgroup. An important example of an arithmetic subgroup is $\operatorname{SL}(n, \mathbb{Z})$. (See $\S 4$ below for a precise definition of arithmetic groups.) Then $\Gamma$ acts properly on $X$ and the quotient $\Gamma \backslash X$ is called an arithmetic locally symmetric space.

The rank of $X$ is defined to be the maximal dimension of flat geodesic subspaces of $X$, which is equal to the $\mathbb{R}$-rank of $\mathbf{G}$, i.e., the maximal dimension of $\mathbb{R}$-split tori of $\mathbf{G}$, and it plays a very important role in the study of $X$ and of lattice subgroups of $G$ acting on $X$. The $\mathbb{Q}$-rank of $\mathbf{G}$ is equal to the maximal dimension of $\mathbb{Q}$-split tori in $\mathbf{G}$ and plays an important role in understanding the geometry of $\Gamma \backslash X$. For example, $\Gamma \backslash X$ is compact if and only if the $\mathbb{Q}$-rank of $\mathbf{G}$ is equal to 0 . Because of this, we also call it the $\mathbb{Q}$-rank of $\Gamma \backslash X$, or

\footnotetext{
${ }^{6}$ We assume that $\mathbf{G}$ is a semisimple linear algebraic group for simplicity. Most of the results stated here work for reductive algebraic groups as well, or with suitable modification. For various applications and induction involving parabolic subgroups, it is important to consider reductive but non-semisimple algebraic groups. For example, $\operatorname{GL}(n, \mathbb{C})$ is a reductive, non-semisimple algebraic group.
} 
rather the $\mathbb{Q}$-rank of $\Gamma$ for convenience, though the terminology may not be so standard. It follows from the definition that the $\mathbb{Q}$-rank of $\Gamma \backslash X$ is less than or equal to the rank of $X$.

For the sake of the discussion below, we can keep the example $\mathbf{G}=$ $\operatorname{SL}(n, \mathbb{C}) \subset \mathrm{GL}(n, \mathbb{C}), n \geq 2$, in mind. Then the $\mathbb{Q}$-rank and $\mathbb{R}$-rank of $\mathbf{G}$ are both equal to $n-1$. For the arithmetic subgroup $\Gamma=\operatorname{SL}(n, \mathbb{Z})$, the quotient $\Gamma \backslash X$ is noncompact. This is consistent with the fact that the $\mathbb{Q}$-rank of $\mathrm{SL}(n, \mathbb{Z})$ is positive.

In the rest of this subsection, we list some properties of arithmetic groups $\Gamma$ roughly according to the following categories:

(a) combinatorial properties,

(b) group theoretical properties,

(c) cohomological properties,

(d) large scale (or asymptotic) properties,

(e) ridigity properties,

(f) classifying spaces and properties of actions.

More details on some of these properties will be provided later.

(a) Combinatorial properties.

(1) $\Gamma$ is finitely generated.

(2) In many cases, for example if $\Gamma=\operatorname{SL}(n, \mathbb{Z}), n \geq 3$, or if $\Gamma$ is equal to the integral points of Chevalley groups ${ }^{7}$ of rank at least $2, \Gamma$ is also boundedly generated. The bounded generation of arithmetic subgroups is closely related to a positive solution of the congruence subgroup problem (see $\S 4.11$ and $[354, \S 6][265$, Theorem D] [349] for the definition and for more details.) But if the $\mathbb{R}$-rank of $\mathbf{G}$ is equal to $1, \Gamma$ is not boundedly generated.

(3) $\Gamma$ is finitely presented. (See $§ 4.11$.

(4) The word problem is solvable for $\Gamma$, and the conjugacy problem of $\Gamma$ is also solvable, but the solvability of the isomorphism problem for arithmetic groups is not known in general. See [159] [160] [161] [4] for related results and references. Some related results on Dehn functions, isoperimetric functions are also known. See [71] for definitions and [197, §17.9] for other references.

(b) Group theoretical properties.

\footnotetext{
${ }^{7}$ Roughly speaking, a Chevalley group is a semisimiple linear algebraic group defined over $\mathbb{Z}$ in the sense that its Lie algebra has a basis, a Chevalley basis, whose structure constants are integers. Once such a Chevalley basis is constructed, the Chevalley group can be defined as the identity component of automorphism group of the Lie algebra and its $\mathbb{Z}$-structure is determined by the $\mathbb{Z}$-structure of the Lie algebra.
} 
(5) $\Gamma$ is residually finite. (See $\S 4.12$.

(6) $\Gamma$ admits torsion-free subgroups of finite index. (See $\S 4.12$.

(7) $\Gamma$ has only finitely many conjugacy classes of finite subgroups. (See $\S 4.12$.

(8) $\Gamma$ is contained in only finitely many other arithmetic subgroups of $G$. (See $\S 4.12$.

(9) If $\mathbf{G}=\mathrm{SL}(n)$ and $\mathbf{G}(\mathbb{Z})=\mathrm{SL}(n, \mathbb{Z})$ and $n \geq 3$, then every arithmetic subgroup $\Gamma$ of $\mathrm{SL}(n, \mathbb{Z})$ is a congruence subgroup, i.e., contains a principal congruence subgroup. This property fails for arithmetic subgroups of $\operatorname{SL}(2, \mathbb{Z})$. In general, for a linear algebraic group $\mathbf{G} \subset G L(n, \mathbb{C})$ defined over $\mathbb{Q}$, the original congruence subgroup problem asks if every arithmetic subgroup $\Gamma \subset \mathbf{G}(\mathbb{Z})$ contains a congruence subgroup. This is equivalent to the condition that the congruence subgroup kernel associated with $\mathbf{G}(\mathbb{Z})$ (or with $\mathbf{G}(\mathbb{Q})$ ) is trivial. More generally, if the congruence subgroup kernel of $\mathbf{G}(\mathbb{Z})$ is finite, we say that the congruence subgroup problem has a positive solution for $\mathbf{G}(\mathbb{Z})$, though not every arithmetic subgroup of $\mathbf{G}(\mathbb{Z})$ contains a congruence subgroup. It is known that if $\mathbf{G}$ is simply connected and absolutely almost simple, if the $\mathbb{R}$-rank of $\mathbf{G}$ (or the rank of the symmetric space $X=G / K$ ) is at least 2, and if the $\mathbb{Q}$-rank of $\mathbf{G}$ is positive, then the congruence subgroup kernel is finite. If the $\mathbb{R}$-rank of $\mathbf{G}$ is equal to 1 , then the congruence subgroup kernel of $\mathbf{G}(\mathbb{Z})$ is infinite in general. The congruence subgroup problem, or the congruence subgroup kernel, is usually formulated and studied for the more general class of $S$-arithmetic subgroups of linear algebraic groups defined over number fields. (See [361] [362, p. 303-304] and $[197, \S 4.4]$ for the most general statements, complete results and more references.)

(10) The Tits alternative holds for $\Gamma$ : every subgroup of $\Gamma$ is either virtually solvable or contains a subgroup isomorphic to the free group $F_{2}$ on two generators. (See §4.14.) For related results on maximal subgroups of infinite index, see the paper [280].

(11) $\Gamma$ is irreducible, i.e., it is not a product of two infinite groups up to finite index, if and only if $\mathbf{G}$ is almost simple over $\mathbb{Q}$. (See [195, Remark 2.5] and [20].)

(12) Assume that $\Gamma$ is irreducible and the $\mathbb{R}$-rank of $G$ (or the rank of $X$ ) is at least 2, then every normal subgroup of $\Gamma$ is either finite or of finite index (Margulis normal subgroup theorem). (See [278] and §4.12.) If the $\mathbb{R}$ rank of $G$ is equal to one, there are in general infinite normal subgroups of infinite index. (See [110].) 
(13) The rank of $\Gamma$ as an abstract group is equal to the real rank of the Lie group $G$, or equivalently the rank of the symmetric space $X=G / K$. (See [22] and references therein.)

(c) Cohomological properties.

(14) The virtual cohomological dimension of $\Gamma$ is finite and is equal to $\operatorname{dim} X-$ $r_{\mathbb{Q}}$, where $r_{\mathbb{Q}}$ is the $\mathbb{Q}$-rank of $\Gamma$ (or $\mathbf{G}$ ). (See $\S 4.18$.)

(15) The cohomology groups $H^{i}(\Gamma, \mathbb{Z})$ and the homology groups $H_{i}(\Gamma, \mathbb{Z})$ are finitely generated in every degree. Cohomology and homology of arithmetic groups have been extensively studied and there is a huge literature on them. (See the most recent survey [379] and the references there.) (See also $§ 4.19$.)

(16) The cohomology ring $H^{*}(\Gamma, \mathbb{Z})$ is finitely generated, which is an analogue of Evens-Venkov theorem for finite groups. (See [357] [213].)

(17) The Euler characteristic of $\Gamma$ can be computed and often expressed in terms of special values of the Riemann zeta function and more general $L$-functions, and Bernoulli numbers in some cases. (See [168] [384, §3.7] [385, §3.1], [79, pp. 253-256], [352].) Basically, this follows from the Gauss-Bonnet formula for the locally symmetric space $\Gamma \backslash X$ and the formula for the volume of $\Gamma \backslash X$ since the Euler-Poincaré measure in the Gauss-Bonnet formula is also invariant under $G$ and hence is proportional to the Haar measure.

(18) $\Gamma$ is a virtual duality group of dimension $\operatorname{dim} X-r_{\mathbb{Q}}$, where $r_{\mathbb{Q}}$ is the $\mathbb{Q}$-rank of $\Gamma$. The dualizing module of $\Gamma$ is the only nonzero reduced homology group of the $\mathbb{Q}$-spherical Tits building $\Delta_{\mathbb{Q}}(\mathbf{G})$, which is an infinite simplicial complex of dimension $r_{\mathbb{Q}}-1$, whose simplices are parametrized by proper $\mathbb{Q}$-parabolic subgroups of $\mathbf{G}$. $\Gamma$ is a virtual Poincaré duality group if and only if $r_{\mathbb{Q}}=0$ is equal to 0 , i.e., if and only if the quotient $\Gamma \backslash X$ is compact. (See $\S 4.19$.)

(19) The cohomology of families of classical arithmetic groups such as $\operatorname{SL}(n, \mathbb{Z})$ stabilizes as $n \rightarrow \infty$. This has important implications for $K$-groups of $\mathbb{Z}$ and rings of integers of number fields. (See [59] and also [197] for references.)

(d) Large scale properties.

(20) If $G$ has no compact factor, then $\Gamma$ is Zariski dense in $G$ (Borel density theorem). (See $\S 4.13$.)

(21) $\Gamma$ grows exponentially, i.e., the number of elements in a ball with respect to any word metric of $\Gamma$ grows exponentially with the radius. (See $\S 4.14$.) 
(22) The asymptotic dimension of $\Gamma$ is finite. Recall that for any metric space $(M, d)$, its asymptotic dimension is the smallest integer $n$ such that for every $R>0$, there exists a covering of $M$ by uniformly bounded sets $\left\{U_{\alpha}\right\}$ such that every geodesic ball of radius $R$ meets at most $n+1$ sets from $\left\{U_{\alpha}\right\}$. This notion was introduced by Gromov. (See [200] for references.)

(23) If $\Gamma$ is cocompact, then the tangent cone (or asymptotic cone) of $\Gamma$ with respect to any word metric is the same as the cone at infinity of the symmetric space $X=G / K$, which is an affine $\mathbb{R}$-building. If $\Gamma$ is irreducible and the rank of $G$ (or $X=G / K$ ) is at least 2, then its tangent cone is not determined yet but is homeomorphic by a Lipschitz map to a subset of an affine $\mathbb{R}$-building, by a result of [267]. (See also [344].) See also [31] [113] for other quasi-isometry invariants of $\Gamma$.

(24) If $\Gamma$ is cocompact, then $\Gamma$ clearly has one end. Otherwise, the number of ends of $\Gamma$ depends on the real rank of $G$. When $G$ has real rank at least $2, \Gamma$ has one end. This follows from the Property $\mathrm{T}$ of $\Gamma$ and a characterization of groups with infinitely many ends by Stallings [394], which implies that a group having Property FA has one end. (See [197, p.154] for more detail.)

\section{(e) Rigidity properties.}

(25) Assume that $\Gamma$ is irreducible and $G$ has trivial center and is not isomorphic to $\operatorname{PSL}(2, \mathbb{R})$. Then for any arithmetic subgroup $\Gamma_{1}$ of a semisimple Lie group $G_{1}$ with trivial center, any isomorphism $\varphi: \Gamma \rightarrow \Gamma_{1}$ extends to an isomorphism $\varphi: G \rightarrow G_{1}$ (Mostow strong rigidity). (See [322].) Furthermore, $\Gamma$ also satisfies the Margulis superrigidity. There is also a lot of work on rigidity for nonlinear actions in the Zimmer program. There are also other rigidity results for measure equivalence and orbit equivalence, and for lattices in more general locally compact groups. (See [278] [432] [315] and the references in [197].) For rigidity results on von Neumann algebras related to arithmetic groups, see [350] and references therein.

(26) When $\Gamma$ is irreducible and $X$ is of rank at least $2, \Gamma$ is quasi-isometry rigid in the sense that any group quasi-isometric to $\Gamma$ is isomorphic to $\Gamma$ up to finite index. (See [122] and the references there.)

(27) The Borel conjecture and the integral Novikov conjectures in $L$-theory (i.e., surgery theory), $K$-theory, and $C^{*}$-algebra theory hold for $\Gamma$. The original Borel conjecture states that if two closed aspherical manifolds are homotopy equivalent, then they are homeomorphic. The Borel conjecture is equivalent to the condition that certain assembly maps are isomorphisms. The isomorphism of an assembly map in each theory is 
sometimes called the Borel conjecture in that theory as well. (See [133] [200] and references therein.)

(28) If $G$ has trivial center and no nontrivial compact factor, then $\Gamma$ is $C^{*}$ simple, i.e., the reduced $C^{*}$-algebra $C_{r}^{*}(\Gamma)$ of $\Gamma$ is simple. (See [34].)

(29) $\Gamma$ is Hopfian, i.e., every surjective homomorphism $\varphi: \Gamma \rightarrow \Gamma$ is an isomorphism, by the general result of Malcev for linear groups. (See $[417, \S 4.4])$.

(30) $\Gamma$ is co-Hopfian, i.e., every injective homomorphism $\varphi: \Gamma \rightarrow \Gamma$ is an isomorphism (See [351].)

(31) If the rank of $G$ is equal to 1 , then $\Gamma$ has Property RD and the BaumConnes conjecture in the theory of $C^{*}$-algebras holds for $\Gamma$ (see [96]) (note that the Baum-Connes conjecture is an analogue of the Borel conjecture and also of the Farrell-Jones conjecture in geometric topology; it asserts that an assembly map for topological $K$-groups in $C^{*}$-algebras is an isomorphism), and $\Gamma$ is also weakly amenable (see [99]). On the other hand, if $G$ is a simple Lie group of rank greater than or equal to 2 and $\Gamma$ is non-uniform, then $\Gamma$ does not have Property RD (see [219] [267]).

(f) Classifying spaces and properties of actions.

(32) There exist $\Gamma$-cofinite universal spaces $\underline{E} \Gamma$ for proper actions of $\Gamma$. (See $\S 4.19$.) If the associated symmetric space $X=G / K$ is linear in the sense that it is a homothety section of a self-adjoint homogeneous cone, then $\Gamma$ admits $\Gamma$-cofinite universal spaces $\underline{E} \Gamma$ of dimension equal to the virtual cohomological dimension of $\Gamma$, which is realized by a spine of $X$, i.e., a $\Gamma$-equivariant deformation retract of $X$. (See [14] and §4.19.)

(33) $\Gamma$ satisfies Property $\mathrm{T}$ when $\Gamma$ is irreducible and $G$ has real rank at least 2. Recall that a group $\Gamma$ satisfies Property $T$ if the trivial representation is isolated in the unitary dual of $\Gamma$, or, equivalently, if whenever $\Gamma$ acts on a Hilbert space unitarily with an almost fixed point, then it has a fixed point. (See [35] for detailed discussions and applications. See also the papers [119] [116].)

(34) $\Gamma$ satisfies Property FA of Serre and hence does not split when $\Gamma$ is irreducible and when $G$ has real rank at least 2. Recall that a group $\Gamma$ has Property FA if every action on a tree has at least one fixed point. If $\Gamma$ acts on a tree but does not have Property FA, then it splits, i.e., admits an amalgam (free product with amalgamation). If $\Gamma$ has Property $\mathrm{T}$, then it has Property FA. (See [6] [279] [416].)

Remark 2.1. In the above discussion, we have assumed that $\Gamma$ is an arithmetic subgroup of a semisimple Lie group $G$. A natural generalization of the class of 
arithmetic subgroups is the class of lattice subgroups of Lie groups, where a discrete subgroup $\Gamma$ of a Lie group $G$ is called a lattice subgroup (or a lattice) if the volume of $\Gamma \backslash G$ is finite with respect to an invariant measure. Then most of the above properties for arithmetic subgroups also hold for lattice subgroups of Lie groups with finitely many connected components.

Arithmetic subgroups of semisimple Lie groups are lattice subgroups. Conversely, by the famous theorem of Margulis on arithmeticity of lattices [278], if $\Gamma$ is an irreducible lattice of a semisimple Lie group $G$ and if the rank of $G$ ( or $X=G / K$ ) is at least 2, then $\Gamma$ is an arithmetic subgroup of $G$. For more details, see $\S 4.2$.

Remark 2.2. Another important and natural generalization of arithmetic subgroups consists of the class of $S$-arithmetic subgroups. Many of the above properties hold for them as well. See $\S 4.3$ for definitions and details.

Though the above list is long, it is sketchy and it is only a list of properties of arithmetic groups known to the author. It is almost surely incomplete. Besides the references for the above properties that were already given, we mention below some books on arithmetic subgroups, discrete subgroups of Lie groups, and related locally symmetric spaces in the order they were first published. We hope that such a list will also give a historical perspective on subjects related to arithmetic subgroups and discrete subgroups.

(1) R. Fricke, F. Klein, Vorlesungen über die Theorie der automorphen Funktionen. Band 1: Die gruppentheoretischen Grundlagen, 1897 [140].

(2) J. Lehner, Discontinuous groups and automorphic functions, 1964 [255].

(3) J. Wolf, Spaces of constant curvature, 1967 [422].

(4) A. Borel, Introduction aux groupes arithmétiques, 1969 [57].

(5) I. M. Gel'fand, M. Graev, I. Pyatetskii-Shapiro, Representation theory and automorphic functions, 1969 [146].

(6) G. Shimura, Introduction to the arithmetic theory of automorphic functions, 1971 [387].

(7) M. Raghunathan, Discrete subgroups of Lie groups, 1972 [360].

(8) G. Mostow, Strong rigidity of locally symmetric spaces, 1973 [322].

(9) W. Magnus, Noneuclidean tesselations and their groups, 1974 [282].

(10) A. Ash, D. Mumford, M. Rapoport, Y. Tai, Smooth compactification of locally symmetric varieties, 1975 [15].

(11) H. Brown, R. Bülow, J. Neubüser, H. Wondratschek, H. Zassenhaus, Crystallographic groups of four-dimensional space, 1978 [78].

(12) J. Humphreys, Arithmetic groups, 1980 [184]. 
(13) M. Vignéras, Arithmétique des algèbres de quaternions, 1980 [409].

(14) A. Beardon, The geometry of discrete groups, 1983 [29].

(15) R. Zimmer, Ergodic theory and semisimple groups, 1984 [432].

(16) L. Charlap, Bieberbach groups and flat manifolds, Universitext. SpringerVerlag, 1986 [94].

(17) S. Krushkal, B. Apanasov, N. Gusevskii, Kleinian groups and uniformization in examples and problems, 1986 [249].

(18) V. Nikulin, I. Shafarevich, Geometries and groups, 1987 [331].

(19) B. Maskit, Kleinian groups, 1988 [283].

(20) G. van der Geer, Hilbert modular surfaces, 1988 [408].

(21) G. Margulis, Discrete subgroups of semisimple Lie groups, 1991 [278].

(22) B. Apanasov, Discrete groups in space and uniformization problems, 1991 [10].

(23) R. Benedetti, C. Petronio, Lectures on hyperbolic geometry, 1992 [37].

(24) S. Katok, Fuchsian groups, 1992 [225].

(25) B. Iversen, Hyperbolic geometry, 1992 [194].

(26) É. Vinberg, O. Shvartsman, Discrete groups of motions of spaces of constant curvature, in Geometry, II, pp. 139-248, 1993 [410].

(27) A. Lubotzky, Discrete groups, expanding graphs and invariant measures, 1994 [266].

(28) V. Platonov, A. Rapinchuk, Algebraic groups and number theory, 1994 [348].

(29) J. Ratcliffe, Foundations of hyperbolic manifolds, 1994 [363].

(30) W. Thurston, Three-dimensional geometry and topology. Vol. 1, 1997 [406].

(31) J. Elstrodt, F. Grunewald, J. Mennicke, Groups acting on hyperbolic space. Harmonic analysis and number theory, 1998 [118].

(32) K. Matsuzaki, M. Taniguchi, Hyperbolic manifolds and Kleinian groups, 1998 [294].

(33) W. Goldman, Complex hyperbolic geometry, 1999 [148].

(34) E. Vinberg, V. Gorbatsevich, O. Shvartsman, Discrete subgroups of Lie groups, in Lie groups and Lie algebras, II, pp. 1-123, 2000 [411].

(35) B. Apanasov, Conformal geometry of discrete groups and manifolds, 2000 [11].

(36) M. Kapovich, Hyperbolic manifolds and discrete groups, 2001 [223].

(37) K. Ohshika, Discrete groups, 2002 [332]. 
(38) W. Fenchel, J. Nielsen, Discontinuous groups of isometries in the hyperbolic plane, 2003 [135].

(39) C. Maclachlan, A. Reid, The arithmetic of hyperbolic 3-manifolds. 2003 [274].

(40) B. Sury, The congruence subgroup problem: an elementary approach aimed at applications, 2003 [398].

(41) L. Keen, N. Lakic, Hyperbolic geometry from a local viewpoint, 2007 [231].

(42) A. Marden, Outer circles. An introduction to hyperbolic 3-manifolds, $2007[277]$.

(43) F. Bonahon, Low-dimensional geometry. From Euclidean surfaces to hyperbolic knots, 2009 [56].

The book [197] contains a lot of references for other topics related to arithmetic groups.

We will define and discuss a portion of these properties in $\S 4$ below, showing how actions on symmetric spaces, reduction theories for arithmetic groups, and compactifications of symmetric and locally symmetric spaces are used to prove some of the above properties, hence emphasizing the importance of interaction between groups and spaces.

\subsection{Properties of actions of arithmetic groups $\Gamma$ on symmetric spaces $X$}

Let $\Gamma \subset G$ be an arithmetic subgroup as above, and $X=G / K$ be a symmetric space of noncompact type with an invariant metric.

(a) Orbits and fixed point sets.

(1) $\Gamma$ acts properly and isometrically on $X$, and $\Gamma \backslash X$ is an orbifold with finitely many singular loci. The orbifold $\Gamma \backslash X$ admits finite smooth covers. This follows from the existence of torsion-free finite index subgroups of $\Gamma$.

(2) For any finite subgroup $F \subset \Gamma$, the fixed point set $X^{F}$ is nonempty and contractible. Hence $X$ is a model of an $\underline{E} \Gamma$-space.

(3) When $\Gamma$ is cocompact, $\Gamma$ with a word metric is clearly quasi-isometric to any $\Gamma$-orbit in $X$ with the induced subspace metric. When $\Gamma$ is irreducible and the rank of $X$ is at least 2, $\Gamma$ with a word metric is quasi-isometric to any $\Gamma$-orbit in $X$ with the induced subspace metric. (See [267].)

(4) Distributions and growths of $\Gamma$-orbits in $X$ can be described. (See [150] and references therein.) 
(b) Boundaries of compactifications of symmetric spaces and $\Gamma$-actions.

(5) As a simply connected nonpositively curved Riemannian manifold, $X$ admits a natural geodesic compactification $X \cup X(\infty)$ by adding the set of equivalence classes of directed geodesics $X(\infty)$ at infinity, where two directed unit speed geodesics $\gamma(t), \gamma^{\prime}(t)$ are equivalent if the distance between them near $+\infty$ is bounded, i.e., $\lim \sup _{t \rightarrow+\infty} d\left(\gamma(t), \gamma^{\prime}(t)\right)<$ $+\infty$. The $\Gamma$-action on $X$ extends continuously to $X \cup X(\infty)$, and the stabilizers of the boundary points in $G$ are exactly the proper parabolic subgroups of $G$. $X$ also admits several other compactifications such as the Satake compactifications on which the $\Gamma$-action extends continuously, and the stabilizers of the boundary points in $G$ are smaller than the parabolic subgroups of $G$ in general. (See $\S 4.16$.)

(6) Compactifications of $X$ such as the geodesic and Satake comapactifications contain distinguished boundary subsets, called the Furstenberg boundaries, that are stable under $\Gamma$. Note that the $\Gamma$-action on the Furstenberg boundaries is not proper. The $\Gamma$-action on the maximal Furstenberg boundary is amenable with respect to the Haar measure and also with respect to any $\Gamma$-quasi-invariant measure. (See [432] [329, Theorem 3.1].) The Furstenberg boundaries and $\Gamma$-actions on them have played a foundational role in the rigidity of arithmetic subgroups and more general lattices of $G$. (See [432] [278].)

(c) Volumes of the quotient space and fundamental sets.

(7) With respect to the invariant Riemannian metric on $X, \Gamma \backslash X$ has finite volume. (See §4.9.)

(8) $\Gamma \backslash X$ is compact if and only if the $\mathbb{Q}$-rank of $\Gamma \backslash X$, i.e., of $\mathbf{G}$, is equal to 0 , or equivariantly, if $\Gamma$ does not contain any nontrivial unipotent element. (See $\S 4.9)$.

(9) If $\Gamma \backslash X$ is compact, then its simplicial volume is positive. (See [251] and [83] for the definition and the precise statement of the result). On the other hand, if the $\mathbb{Q}$-rank of $\Gamma \backslash X$ is at least 3, then the simplicial volume of $\Gamma \backslash X$ is zero. (See [263, Theorem 1.1].) When the $\mathbb{Q}$-rank of $\mathbf{G}$ is equal to 1 or 2 , it is not known whether the simplicial volume of $\Gamma \backslash X$ is positive or not. For some $\mathbb{Q}$-rank 1 locally symmetric spaces including the Hilbert modular varieties, it is known that the simplicial volume is positive [262], and for the Hilbert modular surfaces, the simplicial volume can be computed explicitly using the result in [84].

(10) In the special case where $\Gamma=\operatorname{SL}(n, \mathbb{Z})$ and $X=\mathrm{SL}(n, \mathbb{R}) / \mathrm{SO}(n)$, there is a Mahler compactness criterion for subsets of $\Gamma \backslash X$. This is a founda- 
tional result in the geometry of numbers and in the reduction theory of arithmetic groups. (See [92].)

(11) Fundamental sets (or rough fundamental domains) of the $\Gamma$-action on $X$ can be described in terms of Siegel sets associated with representatives of $\Gamma$-conjugacy classes of $\mathbb{Q}$-parabolic subgroups of $\mathbf{G}$. In some special cases, fundamental domains can be described explicitly, for example, in the theory of Minkowski reduction for $\operatorname{GL}(n, \mathbb{Z})$. (See $\S 4.8$ and $\S 4.9$.)

(12) The volume of $\Gamma \backslash X$ can be computed and can be expressed in terms of special values of the Riemann zeta function or more generally $L$ functions. (See [352].)

(13) The Gauss-Bonnet formula holds for $\Gamma \backslash X$ and can be used to compute the Euler characteristic of arithmetic groups. (See [168] [385, §3].)

(d) Rigidity properties.

(14) Suppose that $\Gamma$ is irreducible and $X$ is not isometric to the Poincare upper half plane. For any locally symmetric space $\Gamma_{1} \backslash X_{1}$ of finite volume, if $\Gamma \backslash X$ and $\Gamma_{1} \backslash X_{1}$ are homotopy equivalent, then they are isometric up to a suitable scaling of the metrics (Mostow strong rigidity). (See [322].)

(15) There are many generalizations of Mostow strong rigidity. In [23], one locally symmetric space is replaced by a nonpositively curved Riemannian manifold without changing the conclusion that the two spaces must be isometric up to scaling. In [254], one locally symmetric space is further replaced by a geodesically complete CAT(0)-space. ${ }^{8}$ In [43], for a compact locally symmetric space of negative sectional curvature, its invariant metric is characterized by the property that it has minimal entropy among all negatively curved metrics of the same volume on it. For other generalizations of Mostow strong rigidity, see the references in [198] and [205].

(16) In [89] and [21], rank rigidity says that any finite volume irreducible nonpositively curved Riemannian manifold of rank at least 2 is a locally symmetric space.

(17) There is also a characterization of irreducible higher rank locally symmetric spaces of finite volume among all irreducible nonpositively curved manifolds of finite volume in terms of bounded cohomology, i.e., vanishing of the vector space of quasi-homomorphisms of the fundamental group. (See [52] [88].)

(e) Compactifications of locally symmetric spaces and ends.

\footnotetext{
${ }^{8} \mathrm{~A} \mathrm{CAT}(0)$-space is a geodesic space such that every triangle in it is thinner than a corresponding triangle of the same side lengths in $\mathbb{R}^{2}$.
} 
(18) Suppose that $\Gamma \backslash X$ is noncompact. Then it admits a Borel-Serre compactification $\overline{\Gamma \backslash X}^{B S}$ such that the inclusion $\Gamma \backslash X \hookrightarrow \overline{\Gamma \backslash X}^{B S}$ is a homotopy equivalence. $\overline{\Gamma \backslash X}^{B S}$ is the quotient of a partial Borel-Serre compactification $\bar{X}^{B S}$ which is a real analytical manifold with corners, and $\Gamma$ acts real-analytically and properly on it. If $\Gamma$ is torsion-free, then $\overline{\Gamma \backslash X}^{B S}$ is a real analytic manifold with corners. (See [62] and [61].) If $\Gamma$ contains torsion elements, then $\overline{\Gamma \backslash X}^{B S}$ is a real analytic compact orbifold.

(19) The locally symmetric space $\Gamma \backslash X$ has one end when the $\mathbb{Q}$-rank of $\Gamma \backslash X$, i.e., of $\mathbf{G}$, is at least 2. When the $\mathbb{Q}$-rank of $\Gamma \backslash X$ is equal to 1 , there are only finitely many ends, and the ends are in one-to-one correspondence with the $\Gamma$-conjugacy classes of $\mathbb{Q}$-parabolic subgroups of G. (See $\S 4.9$.) By passing to subgroups of large finite index, we can get locally symmetric spaces of $\mathbb{Q}$-rank 1 with as many ends as desired.

(20) The partial Borel-Serre compactification $\bar{X}^{B S}$ is a cofinite model of $\underline{E} \Gamma$. (See [206].)

(21) $\Gamma \backslash X$ also admits other compactifications such as the reductive BorelSerre compactification $\bar{X}^{R B S}$, and the Baily-Borel compactification $\overline{\Gamma \backslash X}^{B B}$ when $\Gamma \backslash X$ is Hermitian. (See $\S 4.17$ and [61] for references.)

(22) $\Gamma \backslash X$ admits deformation retracts that are compact submanifolds with corners, i.e., the thick part where the injectivity radius is bounded uniformly from below. This gives a realization of the partial Borel-Serre compactification of $X$ by a subspace of $X$, i.e., the inverse image in $X$ of the thick part of $\Gamma \backslash X$. (See [371].)

(23) If the $\mathbb{Q}$-rank $r_{\mathbb{Q}}$ of $\Gamma \backslash X$ is equal to 1 or if $X$ is a linear symmetric space, then $\Gamma \backslash X$ admits deformation retracts that are of dimension $\operatorname{dim} X-r_{\mathbb{Q}}$, i.e., the virtual cohomological dimension of $\Gamma$. (See $\S 4.19$ for more details and references.) On the other hand, $X$ is a contractible complete Riemannian manifold of smallest dimension on which $\Gamma$ can act by isometries, or is a contractible manifold on which $\Gamma$ acts properly. (See [50].)

(24) When $\Gamma \backslash X$ is a Hermitian arithmetic symmetric space, the Baily-Borel compactification $\overline{\Gamma \backslash X}^{B B}$ is a normal projective variety defined over specific number fields. Let $D$ be the unit disc of $\mathbb{C}$. The Borel extension theorem says that every holomorphic map $D-\{0\} \rightarrow \Gamma \backslash X$ extends to a holomorphic map $D \rightarrow \overline{\Gamma \backslash X}^{B B}$. (See [60].)

(f) Large scale geometry. 
(25) The asymptotic cone (or the tangent space at infinity) of $\Gamma \backslash X$ is a cone over a finite simplicial complex, which is the quotient $\Gamma \backslash \Delta_{\mathbb{Q}}(\mathbf{G})$ of the Tits building $\Delta_{\mathbb{Q}}(\mathbf{G})$. (See [177] [215] [256].)

(26) The rays or EDM (eventually distance minimizing) geodesics of $\Gamma \backslash X$ can be classified and suitable equivalence classes of such geodesics can be identified with boundary points of various compactifications. (See [215].)

(27) The logarithm law for geodesics holds for $\Gamma \backslash X$. This says roughly that from any fixed basepoint, for almost all unit speed geodesics $c(t)$ starting at this point, $\lim \sup _{t \rightarrow+\infty} d\left(c(t), x_{0}\right) / \log t$ exists and is a positive constant depending only on $X$ (See [239] [396].)

(g) Spectral theory of $\Gamma \backslash X$.

(28) When $\Gamma \backslash X$ is noncompact, the Laplace operator associated with the invariant metric has a nonempty continuous spectrum that consists of a union of half lines $[a,+\infty)$, and their generalized eigenfunctions are given by Eisenstein series. This is the famous theory of Eisenstein series that has played a fundamental role in the celebrated Langlands program. The square integrable eigenfunctions are Maass forms and their existence is a subtle problem. (See [252].)

(29) There is a Selberg (or Arthur-Selberg) trace formula relating the spectral data of $\Gamma \backslash X$ to the geometric data of $\Gamma \backslash X$. When $\Gamma \backslash X$ is a hyperbolic surface, the trace formula relates the spectra (both the continuous and the discrete) of the Laplace operator to the lengths of closed geodesics. For general locally symmetric spaces, the geometric side is in terms of orbital integrals. The original motivation of the Selberg trace formula is to prove existence of Maass forms, and the Arthur-Selberg trace formula has been used to prove the Langlands functioriality principle. (See [383] [12].)

(30) A generalized Poisson relation connects sojourn times of scattering geodesics and the singularities of the Fourier transform of the spectral measure. (See [218].) This relation was motivated by the Poisson relation for compact Riemann manifolds in [115].

(h) $L^{2}$-cohomology and intersection cohomology.

(31) When $\Gamma \backslash X$ is a Hermitian locally symmetric space, the Baily-Borel compactification $\overline{\Gamma \backslash X}^{B B}$ is usually a singular projective variety. The intersection cohomology of $\overline{\Gamma \backslash X}^{B B}$ with the middle perversity can be canonically identified with the $L^{2}$-cohomology of $\Gamma \backslash X$, which is known as the 
Zucker conjecture and was proved independently in [264] [373]. See the survey [217] in this volume.

(32) For $p \gg 1$, the $L^{p}$-cohomology group of $\Gamma \backslash X$ can be canonically identified with the cohomology group of the reductive Borel-Serre compactification $\overline{\Gamma \backslash X}^{R B S}$. (See [433].)

We will not discuss in detail most of the above properties, except several properties related to arithmetic groups in $\S 4$.

\subsection{Properties of mapping class groups $\operatorname{Mod}_{g, n}$}

Let $S_{g}$ be a compact oriented surface of genus $g$, and $S_{g, n}$ be a noncompact surface obtained from $S_{g}$ by removing $n$ points. For simplicity, $S_{g, 0}$ is defined to be $S_{g}$. Let $\operatorname{Mod}_{g, n}=\operatorname{Diff}^{+}\left(S_{g, n}\right) / \operatorname{Diff}^{0}\left(S_{g, n}\right)$ be the mapping class group of $S_{g, n}$, where $\operatorname{Diff}^{+}\left(S_{g, n}\right)$ is the group of orientation preserving diffeomorphisms of $S_{g, n}$, and $\operatorname{Diff}^{0}\left(S_{g, n}\right)$ is the identity component of $\operatorname{Diff}^{+}\left(S_{g, n}\right)$, which is also a normal subgroup. $\operatorname{Mod}_{g, n}$ only depends on the pair $g, n$. Let $\mathrm{Homeo}^{+}\left(S_{g, n}\right)$ be the group of orientation preserving homeomorphisms of $S_{g, n}$, and $\operatorname{Homeo}^{0}\left(S_{g, n}\right)$ the identity component. Then the quotient group Homeo $^{+}\left(S_{g, n}\right) /$ Homeo $^{0}\left(S_{g, n}\right)$ is isomorphic to $\operatorname{Mod}_{g, n}$. (See [313].)

Assume that $2 g-2+n>0$. Then $S_{g, n}$ admits complete hyperbolic metrics of finite area. Let $\mathcal{T}_{g, n}$ be the Teichmüller space of marked complete hyperbolic metrics of finite area (or equivalently complex structures) on $S_{g, n}$. Then $\operatorname{Mod}_{g, n}$ acts on $\mathcal{T}_{g, n}$ by changing the markings of the hyperbolic metrics, and the quotient $\operatorname{Mod}_{g, n} \backslash \mathcal{T}_{g, n}$ is the moduli space $\mathcal{M}_{g, n}$ of complete hyperbolic metrics of finite area on $S_{g, n}$, or equivalently the moduli space of compact Riemann surfaces of genus $g$ with $n$ punctures, i.e., the moduli space in algebraic geometry of projective curves over $\mathbb{C}$ with $n$ marked points.

In this subsection, we list some properties of mapping class groups $\operatorname{Mod}_{g, n}$. In the next subsection, we list properties of the action of $\operatorname{Mod}_{g, n}$ on $\mathcal{T}_{g, n}$ and its quotient $\operatorname{Mod}_{g, n} \backslash \mathcal{T}_{g, n} \cong \mathcal{M}_{g, n}$.

(a) Nonisomorphism with arithmetic groups.

(1) $\operatorname{Mod}_{g, n}$ is not isomorphic to any arithmetic subgroup $\Gamma$ of a semisimple Lie group $G$ (see [188]) or more generally to any lattice subgroup of a Lie group with finitely many connected components (see [195] for references.) Stronger rigidity results on chatacterization of locally compact second countable topological groups that contain $\operatorname{Mod}_{g, n}$ as a lattice was obtained in [238, Corollary 1.5].

(2) If $\Gamma$ is an irreducible lattice of a semisimple Lie group of rank at least 2, then any homomorphism $\rho: \Gamma \rightarrow \operatorname{Mod}_{g, n}$ has a finite image. (See [167] 
for references.) On the other hand, the symmetric statement that any homomorphism $\phi: \operatorname{Mod}_{g, n} \rightarrow \Gamma$ has finite image is not true. ${ }^{9}$

\section{(b) Combinatorial properties.}

(3) $\operatorname{Mod}_{g, n}$ is finitely generated. In fact, it is generated by finitely many suitable Dehn twists. (See [188] [127] [54] [67].) But $\operatorname{Mod}_{g, n}$ is not boundedly generated. (See [126].)

(4) $\operatorname{Mod}_{g, n}$ is finitely presented. (See [188] [127].)

(5) $\operatorname{Mod}_{g, n}$ is automatic and hence the word problem is solvable. The conjugacy problem for $\operatorname{Mod}_{g, n}$ is also solvable (See [320] [179] [123].)

(c) Group theoretical properties.

(6) $\operatorname{Mod}_{g, n}$ admits torsion-free subgroups of finite index. (See [188].)

(7) $\operatorname{Mod}_{g, n}$ is residually finite. (See [188].)

(8) $\operatorname{Mod}_{g, n}$ has only finitely many conjugacy classes of finite subgroups. (See [216].)

(9) Every finite subgroup of $\operatorname{Mod}_{g, n}$ can be realized as a subgroup of the automorphism group of a Riemann surface (the Nielsen realization problem). (See [233] [424].)

(10) $\operatorname{Mod}_{g, n}$ satisfies a strong version of the Tits alternative: every subgroup of $\operatorname{Mod}_{g, n}$ is either virtually abelian or contains a subgroup isomorphic to the free group $F_{2}$ on two generators. (See [295] [55] [190] and references.) For more results on subgroups of $\operatorname{Mod}_{g, n}$ and similarities with results for finitely generated linear groups, see [190] and also [232] [340] [321].

(11) $\operatorname{Mod}_{g, n}$ is irreducible, i.e., it is not isomorphic to a product of two infinite groups up to finite index. (See [188].)

(12) The rank of $\operatorname{Mod}_{g, n}$ as an abstract group is equal to 1 [188], but its geometric rank is equal to $3 g-3+n$ [33].

(13) $\operatorname{Mod}_{g, n}$ contains infinite normal subgroups that are of infinite index, i.e., the analogue of the Margulis normal subgroup theorem does not hold. In the case of $\operatorname{Mod}_{g}$, there is a surjective homomorphism $\operatorname{Mod}_{g} \rightarrow \operatorname{Sp}(2 g, \mathbb{Z})$ with an infinite kernel, the Torelli group, which is an infinite normal subgroup. (See [123] for more discussion.)

\footnotetext{
${ }^{9}$ For example, there is a natural surjective homomorphism $\operatorname{Mod}_{g} \rightarrow \operatorname{Sp}(2 g, \mathbb{Z})$. For $n>0$, there is also a surjective homomorphism $\operatorname{Mod}_{g, n} \rightarrow \operatorname{Mod}_{g, n-1}$. (See [169, p. 144].) Given the super-rigidity of higher rank arithmetic irreducible subgroups, it may be natural to conjecture that the first example mentioned is essentially the only case of a homomorphism of $\operatorname{Mod}_{g, n}$ into a semisimple algebraic group with a Zariski dense image.
} 
(14) The congruence subgroup problem for $\operatorname{Mod}_{g, n}$ is solved for some cases when $g \leq 2$ and is still open for higher values of $g$. (See [191] for a precise definition of the congruence subgroup problem and applications, and [364] and references for known results on that problem.)

(d) Cohomological properties.

(15) The virtual cohomological dimension of $\operatorname{Mod}_{g, n}$ is finite and can be written down explicitly. For example, the virtual cohomological dimension of $\operatorname{Mod}_{g}$ is equal to $4 g-5$. (See [169].)

(16) The cohomology and homology groups of $\operatorname{Mod}_{g, n}$ are finitely generated in every degree. (See [169] [216].) A lot of work has been done about these groups. See [169] for a good summary and [124] for some motivation and applications.

(17) The cohomology ring $H^{*}\left(\operatorname{Mod}_{g, n}, \mathbb{Z}\right)$ is finitely generated, which is an analogue of the Evens-Venkov theorem for finite groups. (See [357] [213].)

(18) The Euler characteristic of $\operatorname{Mod}_{g, n}$ can be computed in terms of Bernoulli numbers, or special values of the Riemann zeta function. (See $[169, \S 8]$ [171].)

(19) $\operatorname{Mod}_{g, n}$ is a virtual duality group, but not a virtual Poincaré duality group. Its dualizing module is the only nonzero reduced homology group of the curve complex $\mathcal{C}\left(S_{g, n}\right)$ of $S_{g, n}$, which is an infinite simplicial complex with vertices corresponding to homotopy classes of essential simple closed curves of $S_{g, n}$ and is an analogue of the Tits building of a linear semisimple algebraic group. (See [169] [188] [192].)

(20) The cohomology groups of families of $\operatorname{Mod}_{g, n}$ stabilize as $g, n \rightarrow+\infty$. (See [169, Theorem 6.1].) The stable rational cohomology ring of $\operatorname{Mod}_{g, n}$ is a polynomial ring as conjectured by Mumford. (See [276] [275].)

(21) There exist cofinite universal spaces for proper actions of $\operatorname{Mod}_{g, n}$. (See [216].)

(e) Large scale properties.

(22) $\operatorname{Mod}_{g, n}$ grows exponentially. The fact that $\operatorname{Mod}_{g, n}$ is not virtually abelian and the Tits alternative imply that it grows at least exponentially. By general results [106, p. 181, Remark 53 (iii)], it grows exponentially.

(23) The asymptotic dimension of $\operatorname{Mod}_{g, n}$ is finite. (See [47].)

(24) The maximal topological dimension of locally compact subsets of any asymptotic cone of $\operatorname{Mod}_{g, n}$ and the geometric rank of $\operatorname{Mod}_{g, n}$ are determined in [33]. Every point of any asymptotic cone of $\operatorname{Mod}_{g, n}$ is a global cut-point and the cone is tree-graded. 
(f) Rigidity properties.

(25) $\operatorname{Mod}_{g, n}$ is quasi-isometry rigid in the sense that any group quasi-isometric to $\operatorname{Mod}_{g, n}$ is isomorphic to $\operatorname{Mod}_{g, n}$ up to finite index. (See [166] [32].) It also satisfies the measure equivalence rigidity. (See [236] [237] for detailed statements.)

(26) The analogue of Mostow strong rigidity holds: For any two subgroups of finite index, $\Gamma_{i} \subset \operatorname{Mod}_{g_{i}, n_{i}}, i=1,2$, any isomorphism $\varphi: \Gamma_{1} \rightarrow \Gamma_{2}$ extends to an isomorphism $\varphi: \operatorname{Mod}_{g_{1}, n_{1}} \rightarrow \operatorname{Mod}_{g_{2}, n_{2}}$. (See [195] for references.) This gives a solution to a conjecture in $[191, \S 14]$. There are also analogues of superrigidity results. (See [124] [188] and references there.)

(27) $\operatorname{Mod}_{g, n}$ satisfies the Hopfian and co-Hopfian properties. (See [193].)

(28) $\operatorname{Mod}_{g, n}$ does not have Property T. (See [9] for the general case and [399] for the genus 2 case.)

(29) $\operatorname{Mod}_{g, n}$ satisfies Serre's Property FA and hence does not split. (Recall that a group has Serre's Property FA if every action of this group on a tree has a fixed point.) (see [103].)

(30) $\operatorname{Mod}_{g, n}$ and its subgroups of finite index have one end, using the fact that a group having Property FA does not split and hence has one end, which follows from a characterization of groups with infinitely many ends by Stallings [394].

(31) The integral Novikov conjecture in both $K$ - and $L$-theories, i.e., on the injectivity of the assembly map, holds for $\operatorname{Mod}_{g, n}$. This follows from a general result stating that finite asymptotic dimension implies the validity of integral Novikov conjectures in $K$-, $L$-theories, which also implies the stable Borel conjecture. (See [200] [201] for explanations and the definition of the stable Borel conjecture.)

(32) $\operatorname{Mod}_{g, n}$ is $C^{*}$-simple, i.e., the reduced $C^{*}$-algebra $C_{r}^{*}\left(\operatorname{Mod}_{g, n}\right)$ is simple. (See [72].)

Since $\operatorname{Mod}_{g, n}$ and its finite index subgroups are not virtual Poincaré duality groups and hence cannot be realized as fundamental groups of closed aspherical manifolds, the usual Borel conjecture stating that two closed aspherical manifolds with the same fundamental group are homeomorphic automatically holds for them. (See [136] for an explanation of the Borel conjecture.)

In the above list, we have tried to follow the order of corresponding properties of arithmetic groups. Since the emphasis here is more on global properties of the group $\operatorname{Mod}_{g, n}$, there are many results on properties of individual elements of $\operatorname{Mod}_{g, n}$ that we have not listed here. See [188] [403] [3] and the references there.

Some of the properties related to structures of Teichmüller spaces will be explained in $\S 5$, but they will be even less detailed than for arithmetic groups. 


\subsection{Properties of actions of mapping class groups $\operatorname{Mod}_{g, n}$ on Teichmüller spaces}

Let $\mathcal{T}_{g, n}$ be the Teichmüller space of surfaces of genus $g$ with $n$ punctures, and $\operatorname{Mod}_{g, n}$ the associated mapping class group defined as above.

(a) Orbits and quotients by the mapping class groups.

(1) The Teichmüller space $\mathcal{T}_{g, n}$ is a contractible complex manifold of dimension $3 g-3+n$, and $\operatorname{Mod}_{g, n}$ acts holomorphically and properly on $\mathcal{T}_{g, n}$. (See [327], [187].)

(2) The quotient $\operatorname{Mod}_{g, n} \backslash \mathcal{T}_{g, n}$ is the moduli space $\mathcal{M}_{g, n}$ of hyperbolic metrics on $S_{g, n}$, or equivalently the moduli space of projective curves over $\mathbb{C}$ with $n$ marked points. $\mathcal{M}_{g, n}$ is an orbifold and admits finite smooth covers. The first statement follows from the fact that $\operatorname{Mod}_{g, n}$ acts transitively on the markings of complex structures on $S_{g, n}$, and the second from the existence of torsion-free finite index subgroups of $\operatorname{Mod}_{g, n}$.

(3) For any finite subgroup $F \subset \operatorname{Mod}_{g, n}$, the fixed point set $\mathcal{T}_{g, n}^{F}$ is nonempty and contractible. Hence, $\mathcal{T}_{g, n}$ is a model of $\underline{E} \operatorname{Mod}_{g, n}$.

(4) $\operatorname{Mod}_{g, n}$ is not quasi-isometric to any of its orbits in $\mathcal{T}_{g, n}$ with respect to the Teichmüller metric [126, Theorem 2.1].

(5) Distributions of $\operatorname{Mod}_{g, n}$-orbits and their asymptotic behavior can be described. (See [120] and references therein.)

(b) Volumes of quotients.

(6) $\mathcal{T}_{g, n}$ admits several different metrics that are invariant under $\operatorname{Mod}_{g, n}$ : the incomplete Weil-Petersson metric, and the complete Teichmüller metric, the Bergman metric, the Kobayashi metric, the Carathéodory metric, the Kähler-Einstein metric, the McMullen metric, the Ricci metric, the perturbed Ricci metric (or Liu-Sun-Yau metric). All these complete metrics are quasi-isometric to each other. (See [260] [425]-[427] [299] and references.) There is also the Thurston (or Lipschitz) asymmetric metric. (See [338] for an exposition and references.) The introduction [336] gives brief definitions of most of these metrics.

(7) There are many different properties of metrics on $\mathcal{T}_{g, n}$. The Weil-Petersson is an incomplete Kähler metric which is also geodesically convex in the sense that every two distinct points are connected by a unique geodesic. For a comprehensive study of the Weil-Petersson metric of Teichmüller space, see [427]. The Teichmüller metric is a Finsler metric and is not a CAT(0)-space or Gromov hyperbolic space but it has some properties that resemble a $\operatorname{CAT}(0)$-space, for example, every two points can be connected by a unique Teichmüller geodesic. See [306] [114] [359] [16] [292] 
and the surveys [290] [338] for references. For the Thurston Lipschitz metric, see the survey [338] and [404]. For the McMullen metric, the bottom of the spectrum of the Teichmüller space is positive [299]. For the Ricci and other related metrics, see [260] [261]. See also [336] for an overview of these metrics.

(8) With respect to any of the above metrics, the volume of $\mathcal{M}_{g, n}$ is finite. This follows from the asymptotics (or rather quasi-isometry class) of the metrics near the boundary of $\mathcal{M}_{g, n}$. In fact, the complete metrics mentioned above are quasi-isometric to a Poincaré type metric near the boundary divisors of the Deligne-Mumford $\overline{\mathcal{M}}_{g, n}{ }^{D M}$. (See [260].) The asymptotic behavior of the Weil-Petersson metric is also known. (See [425] [426].)

(9) For any finite index torsion-free subgroup $\Gamma$ of $\operatorname{Mod}_{g, n}$, the simplicial volume of $\Gamma \backslash \mathcal{T}_{g, n}$ is zero when $g \geq 2$, or $g=1$ and $n \geq 3$, and $g=0$ and $n \geq 6$. (See [209] [210].)

(c) Symmetries and compactifications of Teichmüller spaces.

(10) The isometry group of $\mathcal{T}_{g, n}$ with respect to any of the metrics in (6) is discrete and contains $\operatorname{Mod}_{g, n}$ as a subgroup of finite index when $3 g-3+n \geq 2$. More generally, for any complete, finite covolume $\operatorname{Mod}_{g, n^{-}}$ invariant Finsler (or Riemannian) metric on $\mathcal{T}_{g, n}$, when $3 g-3+n \geq 2$, its isometry group contains $\operatorname{Mod}_{g, n}$ as a subgroup of finite index. (See [131, Theorem 1.2].) Except for a few exceptions, the isometry group of the Teichmüller metric and the Weil-Petersson metric of $\mathcal{T}_{g, n}$ is equal to $\operatorname{Mod}_{g, n}^{*}$, which is the degree-two extension of $\operatorname{Mod}_{g, n}$ obtained by including the diffeomorphisms that do not necessarily preserve the orientation of the surface $S_{g, n}$. This is related to the fact that the automorphism group of the curve complex $\mathcal{C}\left(S_{g, n}\right)$ is equal to $\operatorname{Mod}_{g, n}^{*}$ except for a low genus few cases. (See [188] [271] [425] and the references there.)

(11) $\mathcal{T}_{g, n}$ admits several compactifications: (a) the Thurston compactification, (b) the Bers compactification, (c) the Teichmüller ray compactification [234], (d) the harmonic map compactification, (e) the Weil-Petersson visual compactification with respect to the Weil-Petersson metric, (f) the compactification via extremal lengths of essential simple closed curves [144], (g) the horofunction (or Gromov) compactification with respect to the asymmetric Thurston metric [415], (h) the real spectrum compactification of $\mathcal{T}_{g}$ as a semi-algebraic set [82], and there are other compactifications, for example, the compactification in [316] associated with $\Lambda$-trees. (a) The Thurston compactification is defined intrinsically by lengths of simple closed geodesics of the marked hyperbolic Riemann surfaces and 
the $\operatorname{Mod}_{g, n}$-action on $\mathcal{T}_{g, n}$ extends continuously to the Thurston compactification (see [403], [405]). (b) The Bers compactification is the closure of $\mathcal{T}_{g, n}$ under the Bers embedding which realizes $\mathcal{T}_{g, n}$ as a bounded domain in $\mathbb{C}^{3 g-3+n}$ (see [235]). It depends on the choice of a basepoint and the action of $\operatorname{Mod}_{g, n}$ on $\mathcal{T}_{g, n}$ does not extend continuously to this compactification. (c) The Teichmüller ray compactification is obtained by adding a point to each ray in the Teichmüller metric from a fixed basepoint in $\mathcal{T}_{g, n}$. It depends on the basepoint and the action of $\operatorname{Mod}_{g, n}$ on $\mathcal{T}_{g, n}$ does not extend continuously to this compactification either (see [234]). It is important to point out that the boundary of the Teichmüller ray compactification is not equal to the visual sphere (i.e., the space of equivalence classes of Teichmüller rays, where two rays are said to be equlvalent if they stay at a bounded distance), since the latter is nonHausdorff [296]. (d) There is also a compactification of $\mathcal{T}_{g, n}$ by harmonic maps, which is equivariantly homeomorphic to the Thurston compactifcation (see [423] for the definition and the proof of the homeomorphism). (e) The Weil-Petersson visual compactification of $\mathcal{T}_{g, n}$ by adding the visual sphere, i.e., the set of geodesics from a fixed base-point [76] depends on the base-point, and the $\operatorname{Mod}_{g, n}$-action on $\mathcal{T}_{g, n}$ does not extend continuously to the boundary when $3 g-3+n \geq 2$. This visual compactification strictly contains the completion of the Weil-Petersson metric of $\mathcal{T}_{g, n}$, which turns out to be the augmented Teichmüller space [1], a partial compactification of $\mathcal{T}_{g, n}$. (See [291] and [425].) (f) The boundary of the compactification by extremal lengths in [144] (see also [312]) is contained in the boundary of the Thurston compactification. (g) The horofunction (or Gromov) compactification with respect to the Thurston asymmetric metric is equivariantly homeomorphic to the Thurston compactification [415]. (h) The real spectrum compactification is only defined for $\mathcal{T}_{g}$ and dominates the Thurston compactification and has the property that the action of $\operatorname{Mod}_{g}$ on $\mathcal{T}_{g}$ extends continuously to the compactification, and every element in $\operatorname{Mod}_{g}$ has at least one fixed point in the compactification $[82]$.

(12) The action of $\operatorname{Mod}_{g, n}$ on the compact metrizable Hausdorff space of complete geodesic laminations for $S_{g, n}$ is topologically amenable and hence the Novikov conjecture in surgery theory holds for $\operatorname{Mod}_{g, n}$. (See [165] and also [236]).

(d) Fundamental sets.

(13) The quotient $\operatorname{Mod}_{g, n} \backslash \mathcal{T}_{g, n}$ is noncompact if its dimension is positive. The reason is that simple closed curves on hyperbolic surfaces can be pinched and the resulting surfaces have smaller genus and more punctures. 
(14) There is a Mumford compactness criterion for subsets of $\mathcal{M}_{g, n}$, which is an analogue of the Mahler compactness criterion for subsets of the locally symmetric space $\mathrm{SL}(n, \mathbb{Z}) \backslash \mathrm{GL}^{+}(n, \mathbb{R}) / \mathrm{SO}(n)$. (See [324].)

(15) Fundamental sets (or rough fundamental domains) of the $\operatorname{Mod}_{g, n}$-action on $\mathcal{T}_{g, n}$ can be described by Bers sets associated with representatives of $\operatorname{Mod}_{g, n}$-orbits of pants decompositions of $S_{g, n}$. The Minkowski reduction theory for $\operatorname{SL}(n, \mathbb{Z})$ acting on the space of positive definite quadratic forms can be generalized to give an intrinsic fundamental domain for $\operatorname{Mod}_{g, n}$ that is a union of finitely many cells ( $§ 5.11$.)

(16) $\mathcal{T}_{g, n}$ has one end. For any finite index subgroup $\Gamma$ of $\operatorname{Mod}_{g, n}, \Gamma \backslash \mathcal{T}_{g, n}$ has also one end if $3 g-3+n>0$. The former is clear since $\mathcal{T}_{g, n}$ is diffeomorphic to $\mathbb{R}^{6 g-6+2 n}$, and the latter follows from the fact that the curve complex $\mathcal{C}\left(S_{g, n}\right)$ is connected. (See $\S 5.6$.)

(17) When $n>0$, the existence of a $\operatorname{Mod}_{g, n}$-equivariant intrinsic simplicial decomposition of $\mathcal{T}_{g, n}$ and of a $\operatorname{Mod}_{g, n}$-equivariant spine of the right dimension, i.e., equal to the virtual cohomological dimension of $\operatorname{Mod}_{g, n}$, is known [169]. On the other hand, when $n=0$, the existence of a $\operatorname{Mod}_{g^{-}}$ equivariant intrinsic cell decomposition of $\mathcal{T}_{g}$ and a spine of $\mathcal{T}_{g}$ of the right dimension is not known in general. When $g=2$, it is known [367]. A $\operatorname{Mod}_{g}$-equivariant, cocompact deformation retract of $\mathcal{T}_{g}$ is known [216], and a $\operatorname{Mod}_{g}$-equivariant, cocompact deformation retract of $\mathcal{T}_{g}$ of positive codimension is also known [203].

(18) $\mathcal{T}_{g}$ admits a $\operatorname{Mod}_{g}$-equivariant deformation retract, which is a cocompact submanifold with corners of $\mathcal{T}_{g}$ and gives a cofinite model of the universal space $\underline{E} \operatorname{Mod}_{g}$ for proper actions of $\operatorname{Mod}_{g}$. (See [216].)

\section{(e) Large scale geometry.}

(19) The asymptotic cone of $\operatorname{Mod}_{g, n} \backslash \mathcal{T}_{g, n}$ with respect to the Teichmüller metric exists and is equal to the metric cone over a finite simplicial complex, which is the quotient of the curve complex $\mathcal{C}\left(S_{g, n}\right)$ by $\operatorname{Mod}_{g, n}$. (See [257] [129].)

(20) The eventually distance minimizing (EDM) geodesics of $\operatorname{Mod}_{g, n} \backslash \mathcal{T}_{g, n}$ in the Teichmüller metric can be classified and the boundary of the DeligneMumford compactification of $\operatorname{Mod}_{g, n} \backslash \mathcal{T}_{g, n}$ can be described in terms of equivalence classes of these geodesics. (See [128].)

(21) The logarithmic law for geodesics holds for $\operatorname{Mod}_{g, n} \backslash \mathcal{T}_{g, n}$ in the Teichmüller metric. It corresponds to the logarithmic law for noncompact finite volume hyperbolic manifolds. (See [289].)

(f) Compactifications of the quotient. 
(22) $\operatorname{Mod}_{g, n} \backslash \mathcal{T}_{g, n}$ admits a Borel-Serre type compactification $\overline{\operatorname{Mod}_{g, n} \backslash \mathcal{T}_{g, n}} B S$ such that the inclusion $\operatorname{Mod}_{g, n} \backslash \mathcal{T}_{g, n} \hookrightarrow{\overline{\operatorname{Mod}_{g, n} \backslash \mathcal{T}_{g, n}}}^{B S}$ is a homotopy equivalence. It can be taken as the quotient of a Borel-Serre type partial compactification $\overline{\mathcal{T}}_{g, n} B S$ on which $\Gamma$ acts properly. The boundary of $\overline{\mathcal{T}}_{g, n} B S$ is homotopy equivalent to the curve complex $\mathcal{C}\left(S_{g, n}\right)$. (See [188] [210] and §5.6).

(23) The curve complex $\mathcal{C}\left(S_{g, n}\right)$ is an infinite simplicial complex. It has infinite diameter and is a hyperbolic space in the sense of Gromov. (See [130] [188] [307].)

(24) The Borel extension theorem for holomorphic maps from the punctured disk to $\mathcal{M}_{g, n}, f: D-\{0\} \rightarrow \mathcal{M}_{g, n}$, holds for the Deligne-Mumford compactification ${\overline{\mathcal{M}_{g, n}}}^{D M}$, i.e., $f$ extends to a holomorphic map $\hat{f}: D \rightarrow$ $\overline{\mathcal{M}}_{g, n}{ }^{D M}$. (See [186].) If the map $f$ is algebraic, then the extension of $f$ after passing to a finite covering of $D-\{0\}$ is the stable reduction of curves in algebraic geometry. (See [109].)

\section{(g) Cohomological properties.}

(25) The $L^{2}$-cohomology group of $\mathcal{M}_{g, n}=\operatorname{Mod}_{g, n} \backslash \mathcal{T}_{g, n}$ with respect to any of the canonical complete metrics in (6) is isomorphic to the cohomology group of the Deligne-Mumford compactification of $\mathcal{M}_{g, n}$. In fact, for all $p<+\infty$, the $L^{p}$-cohomology group of $\mathcal{M}_{g, n}$ with respect to any of the above canonical complete metrics is also isomorphic to the cohomology group of the Deligne-Mumford compactification of $\mathcal{M}_{g, n}$. (They all define the same $L^{p}$-cohomology groups since they are quasi-isometric.)(See [372] [217].) With respect to the incomplete Weil-Petersson metric, when $p \geq 4 / 3$, the $L^{p}$-cohomology group of $\mathcal{M}_{g, n}$ is also isomorphic to the cohomology group of the Deligne-Mumford compactification of $\mathcal{M}_{g, n}$, but for $p<\frac{4}{3}$, it is isomorphic to the cohomology group of $\mathcal{M}_{g, n}$.

(26) The Gauss-Bonnet formula holds for $\mathcal{M}_{g, n}$ in all the canonical metrics defined in (6) above. (See [214].)

(27) The stable rational cohomology ring of $\mathcal{M}_{g, n}$ is a polynomial ring generated by the Miller-Morita-Mumford $\kappa_{i}$-classes of dimension $2 i$ as conjectured by Mumford. (See [276] [275].)

There are many results on dynamics and properties of elements of $\operatorname{Mod}_{g, n}$ acting on Teichmüller spaces and their boundaries which are not mentioned here. See the papers [188] [32] [166] and the books [334] [335] [124] [403] [3] and the extensive references there for problems and results related to Teichmüller spaces and mapping class groups. 


\subsection{Properties of outer automorphism groups $\operatorname{Out}\left(F_{n}\right)$}

Besides the above two classes of groups, there is a closely related class of groups, $\operatorname{Out}\left(F_{n}\right)=\operatorname{Aut}\left(F_{n}\right) / \operatorname{Inn}\left(F_{n}\right)$, where $F_{n}$ is the free group on $n$ generators, $n \geq 2$. A lot of recent work on $\operatorname{Out}\left(F_{n}\right)$ has been motivated by results on arithmetic groups and $\operatorname{Mod}_{g, n}$. When $n=2$, it is known that $\operatorname{Out}\left(F_{n}\right) \cong \mathrm{GL}(2, \mathbb{Z})$, and $\operatorname{Mod}_{g}=\mathrm{SL}(2, \mathbb{Z})$. This explains the common roots of these three important classes of groups.

We will also mention some properties of other two classes of groups: Coxeter groups and hyperbolic groups. Since these three classes of groups are not our main objects of study, the lists of their properties will not be as exhaustive as the previous two, due to the limit of knowledge of the author.

The following is a partial list of properties of $\operatorname{Out}\left(F_{n}\right)$.

(a) Nonisomorphism with arithmetic groups and mapping class groups.

(1) When $n \geq 3, \operatorname{Out}\left(F_{n}\right)$ is not isomorphic to any arithmetic subgroup of a linear algebraic group (more generally of a lattice subgroup of a Lie group with finitely many connected components) or to a mapping class

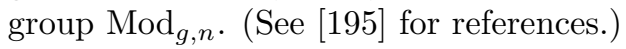

(2) When $n \geq 4$, $\operatorname{Out}\left(F_{n}\right)$ is not linear. (See [137].)

(b) Combinatorial properties.

(3) $\operatorname{Out}\left(F_{n}\right)$ is finitely generated, and explicit generators are known. (See [412].)

(4) $\operatorname{Out}\left(F_{n}\right)$ is not boundedly generated. (See [126, Theorem 3.5].)

(5) $\operatorname{Out}\left(F_{n}\right)$ is finitely presented, and explicit relations are known. (See [413].)

(6) $\operatorname{Out}\left(F_{n}\right)$ is not an automatic group but the word problem for it is solvable. (See [73].)

(c) Group theoretical properties.

(7) $\operatorname{Out}\left(F_{n}\right)$ has only finitely many conjugacy classes of finite subgroups. (See $[412]$.

(8) $\operatorname{Out}\left(F_{n}\right)$ admits torsion-free subgroups of finite index. (See [272, p. 2527].)

(9) $\operatorname{Out}\left(F_{n}\right)$ is residually finite. (See [27] [26].)

(10) A strong version of the Tits alternative holds for $\operatorname{Out}\left(F_{n}\right)$ : every subgroup of $\operatorname{Out}\left(F_{n}\right)$ is either virtually abelian or contains a free subgroup isomorphic to $F_{2}$. (See [51].) 
(11) $\operatorname{Out}\left(F_{n}\right)$ is irreducible, i.e., it is not a product of two infinite groups up to finite index. (See [195] for references).

(d) Cohomological properties.

(12) The virtual cohomological dimension of $\operatorname{Out}\left(F_{n}\right)$ is equal to $2 n-3$. (See [102].)

(13) The cohomology and homology groups of $\operatorname{Out}\left(F_{n}\right)$ are finitely generated in every degree. (See [414].)

(14) The cohomology ring $H^{*}\left(\operatorname{Out}\left(F_{n}\right), \mathbb{Z}\right)$ is finitely generated, which is an analogue of the Evens-Venkov theorem for finite groups. (See [357] [213].)

(15) The Euler characteristic of $\operatorname{Out}\left(F_{n}\right)$ is known for small $n$. On the other hand, there is no simple formula for general $n$. (See $[412, \S 2.2 .6][245$, $\S 7.2])$

(16) The cohomology group of the family $\operatorname{Out}\left(F_{n}\right)$ stabilizes as $n \rightarrow+\infty$ : $H_{i}\left(\operatorname{Out}\left(F_{n}\right)\right)$ is independent of $n$ when $n \geq 2 i+4$. (See [175], and [143] for stability of $H_{i}\left(\operatorname{Aut}\left(F_{n}\right)\right)$.)

(17) $\operatorname{Out}\left(F_{n}\right)$ is a virtual duality group, but not a virtual Poincaré duality group. (See [49] [196].) The dualizing module of $\operatorname{Out}\left(F_{n}\right)$ is not known yet as in the cases of arithmetic groups and mapping class groups. There are several candidates for the analogue of the curve complex and spherical Tits buildings for $\operatorname{Out}\left(F_{n}\right)$. These simplicial complexes have the homotopy type of a bouquet of spheres, i.e., the analogue of the Solomon-Tits theorem for Tits buildings holds [176], but the problem whether their homology group can realize the dualizing module of $\operatorname{Out}\left(F_{n}\right)$ is not clear. (See also [221] [204]).

(18) There exist cofinite universal spaces of proper actions of $\operatorname{Out}\left(F_{n}\right)$ of dimension equal to the virtual cohomological dimension of $\operatorname{Out}\left(F_{n}\right)$, which is equal to $2 n-3$. (See [102] for the contractibility of outer space and its equivariant deformation retraction to its spine, and [420] [248] for the contractibility of fixed point sets of finite subgroups of $\operatorname{Out}\left(F_{n}\right)$.)

(d) Rigidity properties.

(19) For any two finite index subgroups $\Gamma_{i} \subset \operatorname{Out}\left(F_{n_{i}}\right), i=1,2$, every isomorphism $\varphi: \Gamma_{1} \rightarrow \Gamma_{2}$ extends to an isomorphism $\varphi: \operatorname{Out}\left(F_{n_{1}}\right) \rightarrow$ $\operatorname{Out}\left(F_{n_{2}}\right)$. (See [125] and also [195] for references).

(20) $\operatorname{Out}\left(F_{n}\right)$ has Property FA of Serre when $n \geq 3$. (See [103].)

(21) $\operatorname{Out}\left(F_{n}\right)$ and its finite index subgroups are co-Hopfian. (See [74] [125].) 
(22) $\operatorname{Out}\left(F_{n}\right)$ is $C^{*}$-simple, i.e., the reduced $C^{*}$-algebra $C_{r}^{*}\left(\operatorname{Out}\left(F_{n}\right)\right)$ is simple. (See [72].)

(e) Large scale properties.

(23) $\operatorname{Out}\left(F_{n}\right)$ has exponential growth. Since $\operatorname{Out}\left(F_{n}\right)$ is not virtually abelian, the Tits alternative implies that it contains non-abelian free groups and hence it grows at least exponentially. By the general results [106, p. 181, Remark 53 (iii)], it grows exponentially.

(24) Out $\left(F_{n}\right)$ and its subgroups of finite index have one end when $n \geq 3$. See [413, Theorem 3.9]. This also follows from the fact that $\operatorname{Out}\left(F_{n}\right)$ has Property FA as for arithmetic groups and $\operatorname{Mod}_{g, n}$.

There are many results on the dynamics of elements of $\operatorname{Out}\left(F_{n}\right)$ and their actions on the outer spaces and their boundaries which are not listed here. See the survey articles [45] [412] [414] and the paper [220].

Comparing with the lists of properties for arithmetic groups and mapping class groups $\operatorname{Mod}_{g, n}$, it is clear that the following conjectures are reasonable:

(1) The rank of $\operatorname{Out}\left(F_{n}\right)$ as an abstract group is equal to 1.

(2) The asymptotic dimension of $\operatorname{Out}\left(F_{n}\right)$ is finite and hence the integral Novikov conjectures in various theories hold for $\operatorname{Out}\left(F_{n}\right)$.

Since $\operatorname{Out}\left(F_{n}\right)$ and its finite index subgroups are not virtual Poincaré duality groups and hence cannot be realized as fundamental groups of closed aspherical manifolds, the Borel conjecture stating that two closed aspherical manifolds with isomorphic fundamental groups are homeomorphic is automatically satisfied by them.

\subsection{Properties of the outer space $X_{n}$ and the action of Out $\left(\boldsymbol{F}_{n}\right)$ on $\boldsymbol{X}_{n}$}

The analogue of symmetric spaces and Teichmüller spaces for Out $\left(F_{n}\right)$ is the outer space $X_{n}$ of marked metric graphs with fundamental group isomorphic to $F_{n}$, which was introduced in [102]. It is an infinite simplicial complex with some vertices and simplicial faces missing, and $\operatorname{Out}\left(F_{n}\right)$ acts on it simplicially by changing markings of the metric graphs.

Though $\operatorname{Out}\left(F_{n}\right)$ had been studied extensively in combinatorial group theory earlier, the introduction of the outer space $X_{n}$ and the action of $\operatorname{Out}\left(F_{n}\right)$ on it has changed the perspective on $\operatorname{Out}\left(F_{n}\right)$. This is an instance which shows the importance of transformation group theory in understanding properties of groups.

The following is a partial list of properties of the outer space $X_{n}$. 
(a) Orbits of action

(1) $X_{n}$ is a contractible infinite simplicial complex of dimension $3 n-4$. When $n=2$, the underlying space of $X_{n}$ can be identified with the upper-half plane $\mathbf{H}^{2}$. (See [102].)

(2) $\operatorname{Out}\left(F_{n}\right)$ acts simplicially and properly on $X_{n}$.

(3) There are only finitely many $\operatorname{Out}\left(F_{n}\right)$-orbits of simplices in $X_{n}$. (See [102].)

(b) Classifying spaces.

(4) For every finite subgroup of $\operatorname{Out}\left(F_{n}\right)$, its fixed point set in $X_{n}$ is nonempty and contractible. Hence $X_{n}$ is a model of the universal space $\underline{E} \operatorname{Out}\left(F_{n}\right)$ for proper actions of $\operatorname{Out}\left(F_{n}\right)$. (See [420] [248].)

(5) $X_{n}$ admits an $\operatorname{Out}\left(F_{n}\right)$-equivariant deformation retraction onto its spine, which is of dimension $2 n-3$, equal to the virtual cohomological dimension of $\operatorname{Out}\left(F_{n}\right)$, and gives a cofinite model of the universal space $\underline{E} \operatorname{Out}\left(F_{n}\right)$ for proper actions of $\operatorname{Out}\left(F_{n}\right)$ of the smallest possible dimension.

(c) Compactifications.

(6) $X_{n}$ admits a compactification on which the $\operatorname{Out}\left(F_{n}\right)$-action on $X_{n}$ extends continuously. This is an analogue of the Thurston compactification of the Teichmüller space $\mathcal{T}_{g, n}$. (See [101] [102, p. 93] [412] [220].)

(7) $X_{n}$ admits an analogue of the Borel-Serre partial compactification on which $\operatorname{Out}\left(F_{n}\right)$ acts properly and which is $(2 n-5)$-connected at infinity. This was used to prove that $\operatorname{Out}\left(F_{n}\right)$ is a virtual duality group. (See [49].) In [204], there is also a realization of this partial compactification of $X_{n}$ by a truncated subspace $X_{n}(\varepsilon)$ as in the case of the realization of the partial Borel-Serre compactification of a symmetric space by its thick part.

Since the outer space $X_{n}$ is not a manifold, many differential geometric and functional theoretical results for symmetric spaces and Teichmüller spaces have no analogues for $X_{n}$. So far, there is no natural complete metric on $X_{n}$ yet.

There are many other results about $\operatorname{Out}\left(F_{n}\right)$ and outer spaces that we have not mentioned here. See the survey articles [412] [414] [45] and the paper [220]. 


\subsection{Properties of Coxeter groups}

Coxeter groups form a large class of groups that often provide interesting examples or counter-examples for various facts. They are also test groups for important properties. Though other groups discussed in this chapter are also special and serve a similar purpose, many properties of Coxeter groups can be determined explicitly from their generators and relations, i.e., their presentations. In this subsection, we list some of the properties of Coxeter groups related to those discussed earlier for arithmetic groups and $\operatorname{Mod}_{g, n}$.

Briefly, a Coxeter matrix is a symmetric matrix $\left(m_{i j}\right), i, j=1, \cdots, n$, with entries in $\mathbb{N} \cup\{\infty\}$ satisfying the conditions: $m_{i i}=1$, and $m_{i j} \geq 2$ if $i \neq j$. The associated Coxeter group is the group defined by the presentation

$$
\left\langle r_{1}, \cdots, r_{n} \mid\left(r_{i} r_{j}\right)^{m_{i j}}=1, \quad i, j=1, \cdots, n\right\rangle .
$$

In this presentation, when $m_{i j}=\infty$, no relation of the form $\left(r_{i} r_{j}\right)^{m_{i j}}$ is imposed. See [105] for precise definitions and detailed discussions. See also [339] for discussions on related braid groups and Artin groups.

Let $W$ be a Coxeter group. Then it satisfies the following properties.

(a) Combinatorial properties.

(1) $W$ is finitely generated, by definition.

(2) $W$ is finitely presented, by definition.

(3) The word problem is solvable for $W$. (See [105, Theorem 3.4.2] [71, Theorem 1.4, p. 441] [105, p. 5, and Theorem 12.3.3].)

(4) The conjugacy problem is solvable for $W$. (See [246] [18].)

(b) Group theoretical properties.

(5) $W$ is virtually torsion-free. (See [105, Corollary D.1.4].)

(6) $W$ is residually finite. (See [105, Proposition 14.1.11].)

(7) A strong version of the Tits alternative holds for $W$ : any subgroup of $W$ either contains a subgroup isomorphic to the free group $F_{2}$ on two generators or is virtually abelian. (See [105, Proposition 17.2.1].)

(8) $W$ is a CAT(0)-group, i.e., it acts properly, isometrically and co-compactly on a $\mathrm{CAT}(0)$-space. This procedure provides many CAT(0)-groups. (See [105, Chap. 12].)

(c) Cohomological properties.

(9) The cohomology and homology groups of $W$ are finitely generated in every degree. 
(10) The cohomology ring $H^{*}(W, \mathbb{Z})$ is finitely generated, which is an analogue of Evens-Venkov theorem for finite groups. (See [357] [213].)

(11) The Euler characteristic of $W$ can be computed explicitly in terms of the presentation. (See [105, Chapter 16].)

(12) Whether $W$ is a virtual Poincaré duality group or not can be determined explicitly. (See [105, Theorem 10.9.2].)

(13) There exist cofinite spaces $\underline{E} W$ for proper actions of $W$. (See $[105, \mathrm{p}$. 5].)

(d) Large scale properties.

(14) $W$ has either polynomial growth or exponential growth. (See [105, Proposition 17.2.1].)

(15) The growth series of $W$ is a rational function. (See $[105, \S 17.1]$.

(e) Rigidity properties.

(16) For torsion free subgroups of $W$, the Borel conjecture stating that assembly maps are isomorphisms holds, since they can be realized as discrete subgroups of $\mathrm{GL}(n, \mathbb{R})$ for some $n$. (See [133].) A more refined version of the Borel conjecture is the relative Borel conjecture for groups containing torsion elements, and the relative Borel conjecture holds for the whole group $W$. (See [355] [323].)

(17) Infinite Coxeter groups do not have Property T. (See [66].)

(18) Every Coxeter group acts amenably on a compact space. (See [111].)

The natural spaces associated with $W$ are $\mathrm{CW}$ complexes and buildings [105]. They give rise to models of the universal spaces $\underline{E} W$ of proper actions of $W$.

\subsection{Properties of hyperbolic groups}

Another important class of groups consists of hyperbolic groups, which were introduced by Gromov [155] to characterize combinatorially phenomena (or properties) of negative curvature, i.e., fundamental groups of compact negatively curved Riemannian manifolds.

Hyperbolic groups are generic groups among all finitely generated groups in some sense and enjoy many good properties. Arithmetic subgroups of real Lie groups of rank at least 2 belong to the opposite ends of the spectrum of finitely generated groups. Another important related class of groups is the 
class of $C A T(0)$-groups. See [71]. See [68] [69] for a discussion on various important classes of groups and relations between them.

Assume that $\Gamma$ is a finitely generated hyperbolic group.

(a) Combinatorial properties.

(1) $\Gamma$ is finitely generated by assumption.

(2) Any non-elementary hyperbolic group, i.e., not containing a cyclic subgroup of finite index, is not boundedly generated. (See [126, Proposition 3.6].)

(3) $\Gamma$ is finitely presented.

(4) The word problem is solvable for $\Gamma$.

(5) The conjugacy problem is solvable for $\Gamma$. (See [71] for details and references.)

(6) The isomorphism problem is solvable for torsion-free hyperbolic groups. (See [382].)

(b) Group theoretical properties.

(7) A strong version of the Tits alternative holds for $\Gamma$ : every subgroup of a hyperbolic group is either virtually cyclic or contains a subgroup isomorphic to $F_{2}$. (See [71].)

(8) $\Gamma$ admits only finitely many conjugacy classes of finite subgroups.

(9) The cohomology and homology groups $H^{i}(\Gamma, \mathbb{Z}), H_{i}(\Gamma, \mathbb{Z})$ are finitely generated in every degree. Furthermore, $\Gamma$ is of type $F P_{\infty}$.

(10) If $\Gamma$ contains a torsion free subgroup of finite index, then the cohomology ring $H^{*}(\Gamma, \mathbb{Z})$ is finitely generated, which is an analogue of Evens-Venkov theorem for finite groups. (See [357] [213].)

(11) The geometric rank of $\Gamma$ is equal to 1 . (See [77] for the definition of the geometric rank.) The algebraic rank of $\Gamma$ as an abstract group is also equal to 1 . (See [22] for the definition of the algebraic rank).

(c) Large scale properties.

(12) The group $\Gamma$ with a word metric, or, equivalently, its associated Cayley graph, admits a compactification by adding a boundary consisting of equivalence classes of geodesics, called the Gromov boundary. The $\Gamma$-action on $\Gamma$ by multiplication extends continuously to the Gromov compactification. See [222] for an extensive summary on structures of the boundary and actions of $\Gamma$ on this boundary. 
(13) The Martin boundary of $\Gamma$, which describes the cone of positive harmonic functions, is known to be equal to the Gromov boundary. The asymptotic behavior of random walks on $\Gamma$ is known. (See [8] [421].)

(14) Except for the trivial case of finite and virtually cyclic groups, $\Gamma$ has exponential growth. (See [13].)

(15) The growth series of $\Gamma$ is a rational function. (See [72, Theorem 3.1, p. 459].)

(16) The asymptotic dimension of $\Gamma$ is finite. (See [368].)

(d) Cohomological properties.

(17) If $\Gamma$ is virtual torsion-free, then the virtual cohomological dimension of $\Gamma$ is finite, and it is equal to the dimension of the Gromov boundary of $\Gamma$.

(18) There exist cofinite models of universal spaces $\underline{E} \Gamma$ for proper actions of Г. (See [302].)

(19) If $\Gamma$ is torsion-free, then $\Gamma$ is a Poincaré duality group of dimension $n$ if and only if its Gromov boundary has the integral Cech cohomology of $S^{n-1}$, and $\Gamma$ is a duality group of dimension $n$ if its Gromov boundary has the integral Cech cohomology of a bouquet of spheres of dimension $n-1$. (See [53].)

(e) Rigidity properties.

(20) The Borel conjecture and the Farrell-Jones conjecture hold for $\Gamma$. (See [25] [24].)

(21) If $\Gamma$ is torsion-free, then $\Gamma$ is Hopfian, i.e., every epimorphism $\varphi: \Gamma \rightarrow \Gamma$ is an isomorphism. (See [381] [85].)

(22) $\Gamma$ satisfies the Kadison-Kaplansky conjecture. (See [356].)

(23) $\Gamma$ satisfies the Baum-Connes conjecture. (See [304].)

(24) $\Gamma$ is weakly amenable. (See [333] and also [99].)

There are detailed lists of properties of hyperbolic groups and closely related CAT(0) groups in [269].

The natural spaces associated with hyperbolic groups $\Gamma$, which are analogues of symmetric spaces for arithmetic groups and Teichmüller spaces for mapping class groups, are the Rips complexes (or Vietoris-Rips complexes). The Rips complexes have played an important role in studying hyperbolic groups.

In the above discussion, we have emphasized similarities between the five classes of groups. On the other hand, there is an important difference between 
them: the first two classes of groups (i.e., arithmetic groups and mapping class groups $\operatorname{Mod}_{g, n}$ ) act properly on manifolds naturally associated with them while the latter three classes of groups do not have natural proper actions on manifolds.

The orbit spaces in the previous two cases are some of the most important manifolds (or rather orbifolds) in mathematics: arithmetic locally symmetric spaces and moduli spaces of Riemann surfaces (or algebraic curves), and we can study analysis, topology and geometry of these spaces. The interaction between geometry, topology and analysis on these spaces makes them very special and interesting. We hope that the above lists of properties have conveyed some similarities, differences and interaction between these spaces as well.

\section{How discrete groups and proper transformation groups arise}

In this section, we discuss briefly several sources from which discrete groups arise, either as discrete subgroups of topological groups or as discrete transformation groups (i.e., groups acting properly discontinuously on topological spaces).

In some sense, the notion of discrete transformation group is more important than that of discrete group. It is the existence of interesting actions which makes the groups interesting. Group actions also make the spaces interesting. Of course, group actions can also be studied for their own sake.

\subsection{Finitely generated groups, Cayley graphs and Rips complexes}

Probably the most direct way to get discrete groups is to start with a group $\Gamma$ and endow it with the discrete topology. In general, this does not lead to an interesting discrete group since there are no natural topological spaces with reasonable properties ${ }^{10}$ on which such a group acts properly. As emphasized at the beginning of this chapter, group actions are needed to understand the groups and also to make the groups interesting.

But there are exceptions, and these exceptions often give rise to interesting examples.

The first important general case is when $\Gamma$ is a finitely generated group. Let $S \subset \Gamma$ be a finite set of generators that is symmetric in the sense that $\gamma \in S$ if and only if $\gamma^{-1} \in S$. We assume that $S$ does not contain the

\footnotetext{
${ }^{10}$ Some natural properties we expect from these spaces include the fact that they are CW-complexes, locally compact topological spaces, or manifolds.
} 
identity element. Associated with $S$, there is a word metric $d_{S}$ on $\Gamma$ defined by $d_{S}(x, y)=\left|x^{-1} y\right|_{S}$, where $x, y \in \Gamma$, and $\left|x^{-1} y\right|_{S}$ is the word length of the shortest expression of $x^{-1} y$ in terms of the generating set $S$. Clearly left multiplication of $\Gamma$ on $\Gamma$ leaves this metric $d_{S}$ invariant, and $\Gamma$ acts isometrically and properly on $\left(\Gamma, d_{S}\right)$.

On the other hand, $\left(\Gamma, d_{S}\right)$ is a totally disconnected topological space. A closely related connected space is the Cayley graph $\mathcal{G}(\Gamma, S)$. The vertices of $\mathcal{G}(\Gamma, S)$ are the elements of $\Gamma$, and two elements $x, y \in \Gamma$ are connected by an edge if and only if $x^{-1} y \in S$. Then $\mathcal{G}(\Gamma, S)$ is an 1-dimensional $\Gamma$-CW complex. Assume that each edge is given length 1 . Then $\mathcal{G}(\Gamma, S)$ becomes a locally compact geodesic length space, and the natural inclusion $\Gamma \hookrightarrow \mathcal{G}(\Gamma, S)$ gives an isometric embedding of $\left(\Gamma, d_{S}\right)$ into $\mathcal{G}(\Gamma, S)$. The left multiplication of $\Gamma$ on $\Gamma$ extends to an isometric and proper action on $\mathcal{G}(\Gamma, S)$.

If $\Gamma$ is a free nonabelian group $F_{n}$ and $S$ is a minimal symmetric set of generators, then $\mathcal{G}(\Gamma, S)$ is a tree and hence contractible. Otherwise, $\mathcal{G}(\Gamma, S)$ is in general non-contractible.

There is a fattened version of the Cayley graph, called the Rips complex, a finite dimensional $\Gamma-\mathrm{CW}$ complex, ${ }^{11}$ which gives rise to cofinite models of $\underline{E} \Gamma$. (See [71, pp. 468-470].) It is constructed as follows. For any positive integer $d$, define a simplicial complex $\mathcal{R}_{d}(\Gamma, S)$ whose $k$-simplexes consist of $(k+1)$-tuples $\left(\gamma_{0}, \gamma_{1}, \cdots, \gamma_{k}\right)$ of pairwise distinct elements of $\Gamma$ such that for all $0 \leq i \leq j \leq k, d_{S}\left(\gamma_{i}, \gamma_{j}\right) \leq d$. The 1 -skeleton of $\mathcal{R}_{1}(\Gamma, S)$ is equal to the Cayley graph $\mathcal{G}(\Gamma, S)$. It is clear that the action of $\Gamma$ on $\mathcal{G}(\Gamma, S)$ extends to an action on $\mathcal{R}_{d}(\Gamma, S)$ with a compact quotient.

It is easy to see that when $\Gamma$ is finitely presented and $d \gg 1, \mathcal{R}_{d}(\Gamma, S)$ is simply connected. In some cases, for example, when $\Gamma$ is a hyperbolic group in the sense of Gromov, it was proved in [155] (see [332, Proposition 2.68]) that $\mathcal{R}_{d}(\Gamma, S)$ is contractible for $d \gg 1$. It was proved in [302] that for any finite subgroup $F \subset \Gamma$, the fixed point set $\left(R_{d}(\Gamma, S)\right)^{F}$ is nonempty and contractible. Therefore, $\mathcal{R}_{d}(\Gamma, S)$ is a cofinite model of the universal space $\underline{E} \Gamma$ for proper actions of $\Gamma$.

From the point of view of large scale geometry, there is no difference between finitely generated discrete groups and their Cayley graphs. For a systematic study of large scale geometry (or asymptotic geometry) of infinite groups, see [156].

\footnotetext{
${ }^{11}$ The Rips complex is also called the Vietoris-Rips complex, or the Vietoris complex. It was first introduced by Vietoris in 1927. After Rips applied the same complex to the study of hyperbolic groups in the sense of Gromov, it was called the Rips complex and popularized by Gromov in 1987.
} 


\subsection{Rational numbers and $p$-adic norms}

Let $\mathbb{Q}$ be the field of rational numbers. Under the standard embedding $\mathbb{Q} \hookrightarrow \mathbb{R}$, the subspace topology on $\mathbb{Q}$ induced from the usual topology of $\mathbb{R}$ is not discrete. With respect to addition, $\mathbb{Q}$ is not a discrete topological group.

On the other hand, there is a natural topological group $\mathcal{A}$ which is locally compact and contains $\mathbb{Q}$ as a discrete subgroup.

Briefly, for every prime number $p$, there is a $p$-adic metric $d_{p}$ on $\mathbb{Q}$. The completion of $\mathbb{Q}$ with respect to $d_{p}$ is called the field of $p$-adic numbers and denoted by $\mathbb{Q}_{p}$. Let

$$
\mathcal{A}=\mathbb{R} \times \prod_{p} \mathbb{Q}_{p} .
$$

As a ring, $\mathcal{A}$ is called the ring of adeles. Then under the diagonal embedding, $\mathbb{Q} \hookrightarrow \mathcal{A}, \mathbb{Q}$ becomes a discrete subgroup.

Similarly, the multiplicative group of nonzero rational numbers $\mathbb{Q}^{*}$ can be embedded into a locally compact, totally disconnected group $\mathcal{I}$, called the ring of ideles, as a discrete subgroup. Both embeddings are important in number theory. See [146].

The rings $\mathcal{A}$ of adeles and $\mathcal{I}$ of ideles are also related to groups that we are discussing here. For example, given a linear semisimple algebraic group $\mathbf{G}$ defined over $\mathbb{Q}$, we can define $\mathbf{G}(\mathcal{A})$, a locally compact group. Then $\mathbf{G}(\mathbb{Q}) \subset$ $\mathbf{G}(\mathcal{A})$ is a discrete subgroup. For a compact open subgroup $C$ of $\mathbf{G}(\mathcal{A})$, the quotient $\mathbf{G}(\mathbb{Q}) \backslash \mathbf{G}(\mathcal{A}) / C$ is a finite union of locally symmetric spaces $\Gamma \backslash X=$ $\Gamma \backslash \mathbf{G}(\mathbb{R}) / K$ discussed in this chapter.

\subsection{Discrete subgroups of topological groups}

A natural way to produce discrete groups is to take subgroups $\Gamma$ of topological groups $G$ such that the induced subspace topology on $\Gamma$ is discrete. As already said, such subgroups are called discrete subgroups.

An important example is $\mathbb{Z}^{n} \subset \mathbb{R}^{n}$. Another important and related example is $\mathrm{SL}(n, \mathbb{Z}) \subset \mathrm{SL}(n, \mathbb{R})$. These are examples of arithmetic subgroups of linear algebraic groups.

The action of $\Gamma$ on $G$ is proper. More generally, for any compact subgroup $K \subset G$, the natural left action of $\Gamma$ on the homogeneous space $G / K$ is also proper. If $G / K$ admits a left $G$-invariant metric or distance function, then the action is also isometric. A particularly important example is when $G$ is a real Lie group and $G / K$ admits a left $G$-invariant Riemannian metric. Then $\Gamma \backslash G$ and $\Gamma \backslash G / K$ provide many important examples of manifolds and orbifolds. 


\subsection{Fundamental groups and universal covering spaces}

Another important source of proper transformation groups comes from fundamental groups of topological spaces. Let $M$ be a connected topological manifold or more generally a connected and locally path connected topological space. Assume that its fundamental group $\pi_{1}(M)$ is nontrivial. Let $\widetilde{M}$ be the universal covering space of $M$. Then the group $\Gamma=\pi_{1}(M)$ acts properly on $\widetilde{M}$, and the quotient $\Gamma \backslash \widetilde{M}$ is equal to $M$.

If $M$ is a finite connected graph, then $\pi_{1}(M)$ is a free group. If $M$ is a surface of negative Euler characteristic, then $\pi_{1}(M)$ is an infinite surface group.

If $M$ is a complex algebraic variety, then $\pi_{1}(M)$ provides a large natural class of groups. Though algebraic varieties can be constructed easily, properties of their fundamental groups are not easy to describe. It is not easy either to decide whether a group can be realized as such a fundamental group. See the book [7] and the paper [224] for details and references.

Instead of smooth manifolds, we can also consider orbifolds and their fundamental groups in the category of orbifolds.

For algebraic varieties (or rather schemes), we can also take their algebraic (or étale) fundamental groups. See [303].

The monodromy group of some differential equations with regular singularities also gives rise to interesting discrete subgroups [181] [182] [429] [430] [108].

Some standard operations on topological spaces such as direct products, smashed products, connected sums also produce direct products of groups, free products of groups, and amalgamated products of groups.

\subsection{Moduli spaces and Mapping class groups}

Let $S$ be a topological space. A natural topological space associated with $S$ is the space of self-homeomorphisms of $S$, $\operatorname{Homeo}(S) .{ }^{12}$ It is a topological group and is often not discrete. Its identity component $\operatorname{Homeo}^{0}(S)$ is a normal subgroup, and the quotient $\operatorname{Homeo}(S) / \operatorname{Homeo}^{0}(S)$ is called the mapping class group of $S$ and denoted by $\operatorname{Mod}(S)$. Since each element of $\operatorname{Mod}(S)$ represents a connected component, it seems natural to give $\operatorname{Mod}(S)$ the discrete topology.

In general, it is not easy to find a good space on which $\operatorname{Mod}(S)$ acts properly. But some special cases provide important examples.

(a) Let $S=S^{1} \times \cdots \times S^{1}$, a torus of dimension $n$. Consider the moduli space of all marked flat Riemannian metrics on $S$ with total volume 1, where a marking is a choice of a basis of $\pi_{1}(S)$. Any such marked flat manifold

\footnotetext{
${ }^{12}$ Other spaces that can be derived from $S$ are products and quotients of $S$ and various combinations, for example, the symmetric product.
} 
corresponds to a marked lattice $\Lambda$ of $\mathbb{R}^{n}$ of covolume 1 , where a marking on a lattice $\Lambda$ is a choice of a basis. It can easily be seen that this moduli space can be identified with the symmetric space $\operatorname{SL}(n, \mathbb{R}) / \operatorname{SO}(n)$. Then $\operatorname{Mod}(S)$ corresponds to $\operatorname{SL}(n, \mathbb{Z})$ and acts properly on this moduli space, and the quotient is the moduli space of flat Riemannian metrics on $S$ with total volume 1 .

(b) Let $S_{g}$ be a compact orientable surface of genus $g$. Assume that $g \geq 2$. Let $\mathcal{T}_{g}$ be the moduli space of marked hyperbolic metrics on $\Sigma$, where a marking is also a choice of a basis of $\pi_{1}\left(S_{g}\right)$. Then $\operatorname{Mod}_{g}=\operatorname{Mod}\left(S_{g}\right)$ acts properly on $\mathcal{T}_{g}$ and the quotient is the moduli space $\mathcal{M}_{g}$ of hyperbolic metrics on $S_{g}$.

\subsection{Outer automorphism groups}

In group theory, a natural question is this: starting from a group $\Gamma$, how to produce new groups from it?

There are several natural groups associated with $\Gamma$ besides taking products. The first group is the group of automorphisms of $\Gamma$, Aut $(\Gamma)$. Similarly, we can consider the group of inner automorphisms $\operatorname{Inn}(\Gamma)$ and the group of outer automorphisms $\operatorname{Out}(\Gamma)=\operatorname{Aut}(\Gamma) / \operatorname{Inn}(\Gamma)$.

For a countable group $\Gamma, \operatorname{Aut}(\Gamma)$ and $\operatorname{Out}(\Gamma)$ are countable groups and hence can be reasonably considered as discrete groups.

Let $S_{g}$ be a compact orientable surface of genus $g \geq 2$, and $\Gamma=\pi_{1}\left(S_{g}\right)$. Then by the Dehn-Nielsen theorem, Out $(\Gamma)=\operatorname{Mod}_{g}$, the mapping class group. (See [188] [127].)

Let $F_{n}$ be the free group on $n$ generators. Then $\operatorname{Out}\left(F_{n}\right)$ was mentioned before, and the group $\operatorname{Aut}\left(F_{n}\right)$ is also important. The automorphism groups of right-angled Artin groups are closely related to the groups discussed in this chapter. See [95] and references therein.

If $\Gamma=\mathbb{Z}^{n}$, then $\operatorname{Out}\left(\mathbb{Z}^{n}\right)=\operatorname{Aut}\left(\mathbb{Z}^{n}\right)=\operatorname{GL}(n, \mathbb{Z})$. This point of view provides one link between the classes of groups discussed in this chapter. ${ }^{13}$

On the other hand, it is not obvious how to find a space on which $\operatorname{Out}(\Gamma)$ acts properly besides its Cayley graphs.

There are some special cases. One particularly interesting case is when $\Gamma=F_{n}$. Then $\operatorname{Out}\left(F_{n}\right)$ acts properly on the outer space of marked metric graphs whose fundamental group is isomorphic to $F_{n}$. Another important case is that of $\operatorname{Out}\left(\pi_{1}\left(S_{g}\right)\right)$ acting properly on the Teichmüller space $\mathcal{T}_{g}$, the space of marked hyperbolic metrics on the surface $S_{g}$.

\footnotetext{
${ }^{13}$ Unlike the Dehn-Nielsen theorem for compact surfaces, it we take a connected graph whose fundamental group is equal to $F_{n}$, for example, the rose $R_{n}$ with $\mathrm{n}$ petals, and apply the construction of mapping class groups in the previous subsection, we will not get $\operatorname{Mod}\left(R_{n}\right) \cong \operatorname{Out}\left(F_{n}\right)$. It seems natural to consider the following generalization. Let $\operatorname{Homtp}\left(R_{n}\right)$ be the group of all homotopy self-equivalences of $R_{n}$, and the $\operatorname{Homtp}^{0}\left(R_{n}\right)$ its identify component. Then $\operatorname{Homtp}\left(R_{n}\right) / \operatorname{Homtp}^{0}\left(R_{n}\right)$ is a group and should be isomorphic to $\operatorname{Out}\left(F_{n}\right)$.
} 


\subsection{Combinatorial group theory}

Another natural way to construct a discrete group is to specify generators and relations between them.

Any group $\Gamma$ defined by finitely many generators and finitely many relations is countable and hence giving it the discrete topology is natural.

On the other hand, given a finitely presented group, it cannot be decided whether the group is finite or not, nor can it be decided whether it is trivial or not.

Besides Cayley graphs and Rips complexes, it is not easy to construct spaces on which finitely generated groups act.

For most arithmetic groups, it is difficult to find explicit generators and relations. This brings up a natural question: how to effectively describe a group.

Probably the most important class of groups constructed by generators and relations is the class of Coxeter groups. It is probably a miracle that many properties can be deduced from the generators and relations of these groups. Furthermore, there are natural spaces with desirable properties on which Coxeter groups act. See [105] for details. See also [100].

There are other important groups whose properties are understood due to their actions on suitable spaces, for example the Thompson group in [81] and the important right angled Artin groups constructed in [46]. See also the paper [80] and the book [147] for a more systematic study on how topological methods, in particular, actions on CW-complexes, are used to study groups.

\subsection{Symmetries of spaces and structures on these spaces}

Since symmetries in various contexts are described by groups, one reasonable way to construct groups is to consider symmetry group of spaces. The above discussion about $\operatorname{Aut}(\Gamma)$ and $\operatorname{Out}(\Gamma)$ fits well this idea.

Different groups arise when different conditions are imposed, i.e., when different kind of symmetries are considered.

Considering the vector space $\mathbb{R}^{n}$ and all linear transformations on $\mathbb{R}^{n}$, we get the general linear group $\mathrm{GL}(n, \mathbb{R})$. If we consider only those linear transformations that preserve the lattice $\mathbb{Z}^{n}$, then we get the discrete subgroup $\operatorname{GL}(n, \mathbb{Z})$.

Clearly $\mathrm{GL}(n, \mathbb{R})$ acts on $\mathbb{R}^{n}$. On the other hand, this action is not proper. To obtain a natural space on which $\operatorname{GL}(n, \mathbb{R})$ acts properly, we note that linear transformations in $\mathrm{GL}(n, \mathbb{R})$ map $\mathbb{Z}^{n}$ to other lattices of $\mathbb{R}^{n}$, and for any lattice $\Lambda$ in $\mathbb{R}^{n}$, its stabilizer in $\operatorname{GL}(n, \mathbb{R})$ is infinite (in fact, it is an arithmetic subgroup with respect to a suitable $\mathbb{Q}$-structure on the algebraic group $\mathrm{GL}(n, \mathbb{C}))$ and permutes bases of $\Lambda$. Therefore, the space of lattices 
with distinguished bases is a natural space on which $\mathrm{GL}(n, \mathbb{R})$ acts properly and transitively, and $\mathrm{GL}(n, \mathbb{Z})$ also acts properly on this space.

If we consider the group of all isometries of $\mathbb{R}^{n}$, which is generated by translations and rotations, then it acts properly on $\mathbb{R}^{n}$. The subgroup stabilizing the lattice $\mathbb{Z}^{n}$ is a discrete subgroup, and it acts properly discontinuously on $\mathbb{R}^{n}$.

More generally, given a metric space $(X, d)$, its group of isometries $I(X)$ is a topological group, and $I(X)$ acts properly on $X$.

There are two special cases. The first case is when $I(X)$ acts transitively on $X$, and $X$ is a homogeneous space. Symmetric spaces considered above are special examples of homogeneous Riemannian manifolds.

The second case is that of a generic metric space $X$, where $I(X)$ is a trivial or at most a discrete group. For example, suppose that $M$ is a manifold and $\Gamma$ is a discrete group acting properly discontinuously on it by diffeomorphisms. Assume that it acts without fixed points. Take a generic metric on the quotient manifold $\Gamma \backslash M$ and lift it up to $M$. Clearly, this metric on $M$ is invariant under $\Gamma$ and its isometry group is in general equal to $\Gamma$.

It is not easy to find explicit and natural examples of metric spaces for which $I(X)$ is an infinite discrete group. In this sense, it is an interesting fact that the isometry group of the Teichmüller space $\mathcal{T}_{g}$ with respect to the Teichmüller metric or Weil-Petersson metric is equal to the mapping class group $\operatorname{Mod}_{g}$ when $g \geq 3$.

\section{Arithmetic groups}

In this section, we give a formal definition of arithmetic groups or rather arithmetic subgroups, explain concepts related to the properties of arithmetic groups introduced in $\S 2.1$, and indicate briefly how their actions on symmetric spaces can be used to prove some of these properties.

\subsection{Definitions and examples}

The most basic example of an arithmetic group is the subgroup $\mathbb{Z}$ of integers in $\mathbb{R}$. However, $\mathbb{R}$ is not a semisimple Lie group. Groups we study in this chapter are natural generalizations of the arithmetic subgroup $\operatorname{SL}(2, \mathbb{Z}) \subset \mathrm{SL}(2, \mathbb{R})$.

Recall that a subgroup $\mathbf{G}$ of $\mathrm{GL}(n, \mathbb{C})$ is called a linear algebraic group if it is an algebraic variety and if its group operations, i.e., the multiplication $\mathbf{G} \times \mathbf{G} \rightarrow \mathbf{G},(x, y) \mapsto x y$, and the inverse $\mathbf{G} \rightarrow \mathbf{G}, x \mapsto x^{-1}$, are morphisms between algebraic varieties. If the variety $\mathbf{G}$ and the group operations are defined over $\mathbb{Q}$, then $\mathbf{G}$ is said to be defined over $\mathbb{Q}$, and $\mathbf{G}$ is also called a 
$\mathbb{Q}$-linear algebraic group. The notion of being defined over $\mathbb{R}$ can be defined similarly. We note that linear algebraic groups are always defined over $\mathbb{C}$.

Important examples of linear algebraic groups include $\mathrm{SL}(n, \mathbb{C})$, the orthogonal group $\mathbf{O}(Q)$ associated with a quadratic form $Q$ in $n$ variables,

$$
\mathbf{O}(Q)=\left\{g \in \mathrm{GL}(n, \mathbb{C}) \mid Q(g x, g x)=Q(x, x), x \in \mathbb{C}^{n}\right\},
$$

and the symplectic group $\operatorname{Sp}(2 n, \mathbb{C})$ associated with a skew-symmetric bilinear form.

The usual special linear group $\operatorname{SL}(n, \mathbb{C})$ is defined over $\mathbb{Q}$. If the quadratic form $Q$ is defined over $\mathbb{Q}$, then $\mathbf{O}(Q)$ is defined over $\mathbb{Q}$. The same thing is true for the symplectic group.

These examples indicate that algebraic groups often arise from linear transformations that preserve a certain algebraic structure, i.e., the symmetry group of the algebraic structure. This also supports the basic point of transformation group theory in this chapter. If the algebraic structure is defined over $\mathbb{Q}$, then the algebraic group that preserves it is defined over $\mathbb{Q}$.

Given a $\mathbb{Q}$-linear algebraic group $\mathbf{G}$, its $\mathbb{Q}$-locus $\mathbf{G}(\mathbb{Q})$ is well-defined. Since it is also defined over $\mathbb{R}$, its real locus $\mathbf{G}(\mathbb{R})$ is a real Lie group with finitely many connected components and is denoted by $G$, i.e., $G=\mathbf{G}(\mathbb{R})$. For every embedding $\mathbf{G} \subset \mathrm{GL}(n, \mathbb{C})$ defined over $\mathbb{Q}$, we can define $\mathbf{G}(\mathbb{Z})=\mathbf{G}(\mathbb{Q}) \cap$ $\mathrm{GL}(n, \mathbb{Z})$. We emphasize that $\mathbf{G}(\mathbb{Z})$ depends on the embedding of $\mathbf{G}$.

Let $K \subset G$ be a maximal compact subgroup. Then the homogeneous space $X=G / K$ is diffeomorphic to $\mathbb{R}^{n}$, where $n=\operatorname{dim} X$. If $\mathbf{G}$ is a reductive algebraic group, for example, $\mathbf{G}=\operatorname{GL}(n, \mathbb{C})$, then $G=\mathrm{GL}(n, \mathbb{R})$, and $X=$ $\mathrm{GL}(n, \mathbb{R}) / \mathrm{O}(n)$ with any invariant Riemannian metric is a symmetric space of nonpositive curvature. If $\mathbf{G}$ is a semisimple algebraic group, then $X$ is a symmetric space of noncompact type.

Clearly any discrete subgroup $\Gamma \subset G$ acts properly on $G$. However, for some applications, it is more convenient to consider the proper action of $\Gamma$ on $X$. For example, it is known that $X$ is a model of the universal space $\underline{E} \Gamma$-space for proper actions of $\Gamma$ whether $G$ is semisimple or not, but $G$ is not simply connected and hence not contractible and cannot serve as a universal space for $\Gamma$. See $[269]$ for detail.

Definition 4.1. A subgroup $\Gamma \subset G=\mathbf{G}(\mathbb{R})$ is called an arithmetic subgroup if it is contained in $\mathbf{G}(\mathbb{Q})$ and commensurable with $\mathbf{G}(\mathbb{Z})$, i.e., the intersection $\Gamma \cap \mathbf{G}(\mathbb{Z})$ is of finite index in both $\Gamma$ and $\mathbf{G}(\mathbb{Z})$.

Natural examples of arithmetic subgroups include $\mathbf{G}(\mathbb{Z})$ and its subgroups of finite index.

We note that given any linear algebraic group $\mathbf{G} \subset \mathrm{GL}(n, \mathbb{C})$ defined over $\mathbb{Q}$, for any $g \in \mathrm{GL}(n, \mathbb{Q}), g \mathbf{G} g^{-1}$ gives another $\mathbb{Q}$-linear algebraic group isomorphic to $\mathbf{G}$, and their $\mathbb{Q}$ - and $\mathbb{R}$-loci are isomorphic. On the other hand, $g \mathbf{G} g^{-1}(\mathbb{Z})$ and $\mathbf{G}(\mathbb{Z})$ are not isomorphic in general. 
Remark 4.2. A more general definition of arithmetic subgroups of the real Lie group $G=\mathbf{G}(\mathbb{R})$ is as follows. A subgroup $\Gamma \subset G$, which is not necessarily contained in $\mathbf{G}(\mathbb{Q})$, is called an arithmetic subgroup if it is commensurable with $\mathbf{G}(\mathbb{Z})$.

The following fact is clear from the definition.

Proposition 4.3. Let $G=\mathbf{G}(\mathbb{R})$ be the real locus of a linear algebraic group $\mathbf{G}$ as above. If $\Gamma$ is an arithmetic subgroup of $G$ according to Definition 4.1 (or according to the more general one in the above remark), then it is a discrete subgroup of $G$.

If $G$ is a reductive Lie group, then $\Gamma \backslash X$ is usually called an arithmetic locally symmetric space.

It should be emphasized that for a Lie group $G$, its arithmetic subgroups depend on the $\mathbb{Q}$-structure of $G$, i.e., on the existence of a $\mathbb{Q}$-linear algebraic group $\mathbf{G}$ whose real locus is equal to $G$. Different $\mathbb{Q}$-structures usually give rise to non-commensurable arithmetic subgroups. For example, $\operatorname{SL}(2, \mathbb{R})$ admits arithmetic subgroups $\Gamma$, for example, $\operatorname{SL}(2, \mathbb{Z})$, such that $\Gamma \backslash \operatorname{SL}(2, \mathbb{R})$ is noncompact, and other arithmetic subgroups $\Gamma^{\prime}$ such that $\Gamma^{\prime} \backslash \mathrm{SL}(2, \mathbb{R})$ is compact.

Remark 4.4. One good example to illustrate the notion of $\mathbb{Q}$-structure is to consider lattices $\Lambda$ of $\mathbb{R}^{n}$ and $\mathbb{Q}$-structures on $\mathbb{R}^{n}$. Each lattice $\Lambda$ defines a $\mathbb{Q}$-structure on $\mathbb{R}^{n}$, i.e., a $\mathbb{Q}$-linear subspace of dimension $n$. Let $v_{1}, \cdots, v_{n}$ be a basis of $\Lambda$. Then $\mathbb{Q} v_{1}+\cdots+\mathbb{Q} v_{n}$ defines a $\mathbb{Q}$-linear subspace $V$ of $\mathbb{R}^{n}$ such that $V \otimes_{\mathbb{Q}} \mathbb{R}=\mathbb{R}^{n}$. Two lattices $\Lambda_{1}$ and $\Lambda_{2}$ define the same $\mathbb{Q}$-structure if and only if $\Lambda_{1} \cap \Lambda_{2}$ is also a lattice.

Remark 4.5. Another definition of arithmetic groups is as follows. It looks more general at first sight, but turns out to be the same by using the functor of restriction of scalars (see [348] for example). Let $k$ be a number field, i.e., a field that is a finite extension of $\mathbb{Q}$. Let $\mathcal{O}_{k}$ be its ring of integers. Suppose that $\mathbf{G} \subset \mathrm{GL}(n, \mathbb{C})$ is a linear algebraic group defined over $k$. Then any subgroup $\Gamma$ of $\mathbf{G}(k)$ commensurable with $\mathbf{G}\left(\mathcal{O}_{k}\right)$ is called an arithmetic subgroup of G. To realize $\Gamma$ as a discrete subgroup of a real Lie group, we need to use the product of $\mathbf{G}\left(k_{\nu}\right)$, where $\nu$ runs over all real and complex embeddings, or Archimedean places of $k$. Embedding into any one of the factors will not give a discrete subgroup in general. The arithmetic subgroups defined in this more general case are also commensurable with "integral" elements.

Remark 4.6. Given any Lie group $H$ with finitely many connected components, it is in general not true that $H$ is the real locus of a $\mathbb{Q}$-linear algebraic group G. For example, any Lie group that is not linear, i.e., that cannot be embedded into $\mathrm{GL}(n, \mathbb{R})$ will provide such an example. Alternatively, suppose 
that $\mathbf{G}$ is a $\mathbb{Q}$-simple linear algebraic group such that its real locus $G$ can be written as a product $G=G_{1} \times G_{2}$ such that $G_{1}$ is noncompact and $G_{2}$ is compact and has positive dimension. Take $H=G_{1}$. Then $H$ is often not the real locus of a $\mathbb{Q}$-linear algebraic group. We can also take $H$ to be the product of $G$ with a compact Lie group that is not linear. For such a Lie group $H$ that differs from the real locus $G=\mathbf{G}(\mathbb{R})$ of a linear algebraic group $\mathbf{G}$ by compact Lie groups, arithmetic subgroups are defined as follows. A discrete subgroup $\Gamma_{H}$ of $H$ is called an arithmetic subgroup if there exists a $\mathbb{Q}$-linear algebraic group $\mathbf{G}$ and a Lie group homomorphism $\varphi: G \rightarrow H$ with compact kernel and compact cokernel and an arithmetic subgroup $\Gamma_{G} \subset G$ such that $\varphi\left(\Gamma_{G}\right)$ is commensurable with $\Gamma_{H}$. For convenience, we call such a $\mathbb{Q}$-linear algebraic group $\mathbf{G}$ and a Lie group homomorphism $\varphi: G \rightarrow H$ a $\mathbb{Q}$-structure on $H$. In general, different $\mathbb{Q}$-structures on $H$ give rise to non-commensurable classes of arithmetic groups. For example, the discussions in Remark 4.4 about $\mathbb{Q}$-structures and lattices in $\mathbb{R}^{n}$ illustrate this point.

A natural question concerns the size of arithmetic subgroups $\Gamma$ relative to the ambient Lie groups $G$. For this purpose, we introduce some definitions.

Definition 4.7. A discrete subgroup $\Gamma$ of a Lie group $G$ with finitely many connected components is called a lattice (or a lattice subgroup) if with respect to any left invariant Haar measure on $G$, the volume of $\Gamma \backslash G$ is finite.

If $\Gamma$ is a lattice of $G$, then the locally homogeneous space $\Gamma \backslash X$ with respect to any invariant metric has finite area, where $X=G / K$ as above.

Definition 4.8. A discrete subgroup or lattice $\Gamma$ of a Lie group $G$ is called a cocompact (or uniform) lattice if the quotient $\Gamma \backslash G$ is compact.

The arithmetic subgroup $\mathbb{Z}$ is a cocompact lattice of $\mathbb{R}$. We note that in order to view $\mathbb{C}$ as a linear algebraic group, we identify it with the unipotent linear algebraic group of upper triangular $2 \times 2$ matrices with 1 s along the diagonal. Then its real locus is $\mathbb{R}$ and $\mathbb{Z}$ is an arithmetic subgroup. More generally, every lattice of $\mathbb{R}^{n}$ is cocompact.

The arithmetic subgroup $\{ \pm 1\}$ of $G L(1, \mathbb{R})=\mathbb{R}-\{0\}$ is not a lattice. The arithmetic subgroup $\mathrm{GL}(2, \mathbb{Z})$ is not a lattice of $\mathrm{GL}(2, \mathbb{R})$ either.

The following results hold (see [360]).

Proposition 4.9. If $G$ is a nilpotent Lie group, then every arithmetic subgroup $\Gamma$ of $G$ is a uniform lattice.

For the semisimple case, the situation is more complicated and hence more interesting. 
Proposition 4.10. If $G$ is a semisimple Lie group, then every arithmetic subgroup $\Gamma$ of $G$ is a lattice.

This is an important consequence of the reduction theory for arithmetic groups discussed below. We note that $\mathrm{GL}(n, \mathbb{Z})$ is not a lattice of $\operatorname{GL}(n, \mathbb{R})$, but $\mathrm{SL}(n, \mathbb{Z})$ is a lattice in $\mathrm{SL}(n, \mathbb{R})$.

The basic question of when an arithmetic subgroup of a semisimple Lie group is a uniform lattice is answered by the following result (see [57] [197] for references).

Proposition 4.11. Assume that $\mathbf{G}$ is a semisimiple linear algebraic group. Then an arithmetic subgroup $\Gamma$ of the real locus $G=\mathbf{G}(\mathbb{R})$ is a uniform lattice of $G$ if and only if $\Gamma$ does not contain any nontrivial unipotent element, which is equivalent to the condition that the $\mathbb{Q}$-rank of $\mathbf{G}$ is equal to 0.

For example, $\mathrm{SL}(2, \mathbb{Z})$ contains many unipotent elements such as $\left(\begin{array}{ll}1 & b \\ 0 & 1\end{array}\right)$, where $b \in \mathbb{Z}$, and hence $\mathrm{SL}(2, \mathbb{Z})$ is a non-uniform arithmetic subgroup of $\mathrm{SL}(2, \mathbb{R})$. Similarly, $\mathrm{SL}(n, \mathbb{Z})$ is a non-uniform arithmetic subgroup of $\mathrm{SL}(n, \mathbb{R})$. Though it is not easy to see it explicitly, $\mathrm{SL}(n, \mathbb{R})$ admits uniform arithmetic subgroups with respect to different $\mathbb{Q}$-structures on $\mathrm{SL}(n, \mathbb{R})$ (or $\mathrm{SL}(n, \mathbb{C})$ ).

In fact, we have the following result of Borel [58].

Proposition 4.12. Every connected semisimple Lie group $G$ contains uniform arithmetic subgroups with respect to suitable $\mathbb{Q}$-structures on $G$.

The basic idea of Proposition 4.12 is to make use of $\mathbb{Q}$-bases of the Lie algebra $\mathfrak{g}$ of $G$, i.e., bases such that the structure constants are rational numbers, to construct a form of $\mathfrak{g}$ over a totally real number field $E$ of degree strictly greater than 1 such that under any non-identity embedding of $E$ into $\mathbb{R}, \mathfrak{g}$ is a compact form of the complex Lie algebra $\mathfrak{g} \otimes \mathbb{C}$. Then the compactness criterion in Corollary 4.36 below shows that the arithmetic subgroups defined with respect to the number field $E$ are uniform. See [58, p. 116 and Proposition 3.8] for more detail.

It is perhaps worthwhile to point out that $G$ also admits different $\mathbb{Q}$ structures which admit non-uniform arithmetic subgroups. They are easier to see for classical groups such as $\operatorname{SL}(n, \mathbb{C})$ and $\operatorname{Sp}(2 n, \mathbb{C})$ etc. In general, they can be constructed by the Chevalley basis of the Lie algebra $\mathfrak{g}$, or rather from the arithmetic subgroups of the Chevalley group associated with the Lie algebra $\mathfrak{g}$.

For example, consider the two quadratic forms $Q_{1}\left(x_{1}, \cdots, x_{n}\right)=x_{1}^{2}+\cdots+$ $x_{n-1}^{2}-x_{n}^{2}$ and $Q_{2}\left(x_{1}, \cdots, x_{n}\right)=x_{1}^{2}+\cdots+x_{n-1}^{2}-a x_{n}^{2}$, where $a$ is a positive integer such that $Q_{2}\left(x_{1}, \cdots, x_{n}\right)=0$ has no nontrivial integral solution. They define two $\mathbb{Q}$-linear algebraic groups $\mathbf{G}_{1}=O\left(Q_{1}\right)$ and $\mathbf{G}_{2}=O\left(Q_{2}\right)$. The 
quadratic forms are not isomorphic over $\mathbb{Q}$, but $\mathbf{G}_{1}(\mathbb{R}) \cong \mathbf{G}_{2}(\mathbb{R})$. Let $G=$ $\mathbf{G}_{1}(\mathbb{R}) \cong \mathbf{G}_{2}(\mathbb{R})$. Arithmetic subgroups of $G$ with respect to the $\mathbb{Q}$-structure from $\mathbf{G}_{1}$ are not uniform discrete subgroups, but arithmetic subgroups of $G$ with respect to the $\mathbb{Q}$-structure from $\mathbf{G}_{2}$ are uniform discrete subgroups.

\subsection{Generalizations of arithmetic groups: non-arithmetic lattices}

Arithmetic subgroups of Lie groups are natural and provide a large class of lattice subgroups. On the other hand, the class of lattices is strictly larger than the class of arithmetic subgroups of semisimple Lie groups.

Recall that a Fuchsian group is said to be of the first kind if its limit set is equal to the whole boundary $\mathbf{H}^{2}(\infty)$. Otherwise it is said to be of the second kind. A lattice of $\operatorname{SL}(2, \mathbb{R})$ or $\operatorname{PSL}(2, \mathbb{R})$ is a Fuchsian group of the first kind. For a finitely generated Fuchsian group, the converse is also true. On the other hand, most Fuchsian groups of the first kind are not arithmetic subgroups for the reason that there are uncountably many Fuchsian groups of the first kind, but only countably many arithmetic subgroups of $\mathrm{SL}(2, \mathbb{R})$. In some sense, Teichmüller theory was created to study these non-arithmetic Fuchsian groups. This adds another link between the two classes of groups in the title of this chapter.

Though there is abundant supply of non-arithmetic Fuchsian groups, it is not obvious how to construct them explicitly. One important class consists of Hecke triangle groups. In fact, most of the Hecke triangle groups are not arithmetic groups.

Recall that for every integer $q \geq 3$, there is a Hecke triangle subgroup $\Gamma_{q}$ of $\mathrm{SL}(2, \mathbb{R})$ generated by $S_{q}=\left(\begin{array}{cc}1 & 2 \cos \pi / q \\ 0 & 1\end{array}\right)$ and $T=\left(\begin{array}{cc}0 & -1 \\ 1 & 0\end{array}\right)$. Except for $q=3,4,6, \Gamma$ is not an arithmetic subgroup, i.e., not commensurable with $\mathrm{SL}(2, \mathbb{Z})$. (See for example, [400] [178] [241]). For relations between Hecke triangle subgroups and Teichmüller theory, see [174].

The isometry group $\mathrm{SO}(n, 1)$ of the real hyperbolic space $\mathbf{H}^{n}$ of dimension $n$ also contains many non-arithmetic lattices [157].

Non-arithmetic lattices only occur in rank 1 semisimple Lie groups. More precisely, the famous arithmeticity theorem of Margulis (see [278], and [198] and the references there) says that if $G$ is a semisimple Lie group of rank at least two and $\Gamma$ is an irreducible lattice of $G$, then $\Gamma$ is an arithmetic subgroup with respect to a suitable $\mathbb{Q}$-structure on $G$. Among rank 1 semisimple Lie groups, the question of arithmeticity of lattices is open only for $\mathrm{SU}(n, 1)$ when $n$ is at least 4. For a survey of some geometric constructions of lattices in $\mathrm{SU}(3,1)$, see [341], and for some constructions of lattices, in $\mathrm{SO}(n, 1)$, for example by reflections, see [410]. 
Lattices of semisimple Lie groups share many properties of arithmetic groups listed in $\S 2.1$. In fact, all the properties listed there hold for them. The basic reason is that an analogue of the reduction theory for arithmetic groups holds for lattices of rank 1 semisimple Lie groups [145] and hence the structure at infinity of associated locally symmetric spaces can be understood.

Remark 4.13. Let $G$ be a semisimple Lie group, $X=G / K$ be the associated symmetric space with an invariant Riemannian metric, and $\Gamma \subset G$ be a lattice. Then $\Gamma \backslash X$ is a locally symmetric space of finite volume. The spectral theory of the Laplace operator of $\Gamma \backslash X$, in particular the question of existence of square integrable eigenfunctions, depends on whether $\Gamma$ is arithmetic or not. This is an instance where whether a lattice $\Gamma$ is arithmetic or not makes a big difference. See [178] [241] and the references there for the Phillips-Sarnak conjecture on existence of square integrable eigenfunctions. Unlike the case of arithmetic locally symmetric spaces $\Gamma \backslash X$ whose volumes can be computed in terms of special values of the Riemann zeta function or L-functions, there is no such formula for non-arithmetic locally symmetric spaces.

\subsection{Generalizations of arithmetic groups: $S$-arithmetic subgroups}

Another generalization of arithmetic subgroups consists of $S$-arithmetic subgroups. The reason why it is a natural generalization is the following consideration. Take any set of finitely many elements $\gamma_{1}, \cdots, \gamma_{m} \in \mathrm{GL}(n, \mathbb{Q})$ and let $\Gamma$ be the subgroup $\left\langle\gamma_{1}, \cdots, \gamma_{m}\right\rangle$. If some of the matrix entries of $\gamma_{1}, \cdots, \gamma_{m}$ are not integral, then $\Gamma$ is not a discrete subgroup of $\operatorname{GL}(n, \mathbb{R})$ in general. (Note that $\Gamma$ might be a discrete subgroup of $\operatorname{GL}(n, \mathbb{R})$. For example, any hyperbolic element $\gamma$ of $\mathrm{SL}(2, \mathbb{R})$ generates a discrete cyclic subgroup of $\mathrm{SL}(2, \mathbb{R})$, and this fact is independent of whether $\gamma$ is integral or not.)

As emphasized in the introduction and Section 3, it is important and fruitful to realize such natural groups $\Gamma$ as discrete subgroups of some locally compact topological groups which are similar to Lie groups in some sense. Let $p_{1}, \cdots, p_{k}$ be the set of primes that occur in the denominators of the matrix entries of $\gamma_{1}, \cdots, \gamma_{m}$. Each prime $p_{i}$ gives a completion $\mathbb{Q}_{p_{i}}$ of $\mathbb{Q}$. Note that $\mathbb{R}$ is the completion of $\mathbb{Q}$ corresponding to $\infty$. Let $S=\left\{\infty, p_{1}, \cdots, p_{k}\right\}$ be a finite set of places of $\mathbb{Q}$. (Note that a place of a field is an equivalence class of valuations of the field.)

Define the ring $\mathbb{Z}_{S}$ of $S$-integers to consist of rational numbers whose denominators contain only primes from $p_{1}, \cdots, p_{k}$. It is also denoted by $\mathbb{Z}\left[\frac{1}{p_{1}}, \cdots, \frac{1}{p_{k}}\right]$.

It is clear that $\Gamma=\left\langle\gamma_{1}, \cdots, \gamma_{m}\right\rangle$ is contained in $\operatorname{GL}\left(n, \mathbb{Z}\left[\frac{1}{p_{1}}, \cdots, \frac{1}{p_{k}}\right]\right)$. It

is also clear that under the diagonal embedding, $\operatorname{GL}\left(n, \mathbb{Z}\left[\frac{1}{p_{1}}, \cdots, \frac{1}{p_{k}}\right]\right)$ is a 
discrete subgroup of $\mathrm{GL}(n, \mathbb{R}) \times \mathrm{GL}\left(n, \mathbb{Q}_{p_{1}}\right) \times \cdots \times \mathrm{GL}\left(n, \mathbb{Q}_{p_{k}}\right)$. Therefore, we have realized $\Gamma$ as a discrete subgroup of the locally compact topological group $\operatorname{GL}(n, \mathbb{R}) \times \operatorname{GL}\left(n, \mathbb{Q}_{p_{1}}\right) \times \cdots \times \mathrm{GL}\left(n, \mathbb{Q}_{p_{k}}\right)$.

Given any linear algebraic group $\mathbf{G} \subset \operatorname{GL}(n, \mathbb{C})$ defined over $\mathbb{Q}$, there is a $\operatorname{subgroup} \mathbf{G}\left(\mathbb{Z}\left[\frac{1}{p_{1}}, \cdots, \frac{1}{p_{k}}\right]\right)=\mathbf{G}(\mathbb{Q}) \cap \mathrm{GL}\left(n, \mathbb{Z}\left[\frac{1}{p_{1}}, \cdots, \frac{1}{p_{k}}\right]\right)$ of $\mathbf{G}(\mathbb{Q})$ and $\mathbf{G}(\mathbb{R})$.

Definition 4.14. A subgroup of $\mathbf{G}(\mathbb{Q})$ is called an $S$-arithmetic subgroup if it is commensurable with $\mathbf{G}\left(\mathbb{Z}\left[\frac{1}{p_{1}}, \cdots, \frac{1}{p_{k}}\right]\right)$.

Proposition 4.15. Under the diagonal embedding, every S-arithmetic subgroup of $\mathbf{G}$ is a discrete subgroup of the locally compact topological group $\mathbf{G}(\mathbb{R}) \times \mathbf{G}\left(\mathbb{Q}_{p_{1}}\right) \times \cdots \times \mathbf{G}\left(\mathbb{Q}_{p_{k}}\right)$.

Remark 4.16. The set $S$ of places of $\mathbb{Q}$ is exactly of the right size so that the product $\mathbf{G}(\mathbb{R}) \times \mathbf{G}\left(\mathbb{Q}_{p_{1}}\right) \times \cdots \times \mathbf{G}\left(\mathbb{Q}_{p_{k}}\right)$ contains $S$-arithmetic subgroups $\Gamma$ as discrete subgroups. Clearly adding more places will still preserve the discreteness of the image of the diagonal embedding of $\Gamma$. Note that if $\mathbf{G}$ is a semisimple linear algebraic group, then any $S$-arithmetic subgroup $\Gamma$ is a lattice of $\mathbf{G}(\mathbb{R}) \times \mathbf{G}\left(\mathbb{Q}_{p_{1}}\right) \times \cdots \times \mathbf{G}\left(\mathbb{Q}_{p_{k}}\right)$ with respect to the Haar measure on the product, and hence adding more places will produce an ambient group which is too big in some sense.

Since $\mathbb{Z}\left[\frac{1}{p_{1}}, \cdots, \frac{1}{p_{k}}\right]$ is not a finitely generated abelian group, it is not true that for any $\mathbb{Q}$-linear algebraic group $\mathbf{G}$, its $S$-arithmetic subgroups, in particular, $\mathbf{G}\left(\mathbb{Z}\left[\frac{1}{p_{1}}, \cdots, \frac{1}{p_{k}}\right]\right)$, are finitely generated. Many other properties of arithmetic subgroups listed in $\S 2.1$ do not hold for them.

If $\mathbf{G}$ is semisimple, then $S$-arithmetic subgroups are finitely generated and finitely presented, and all other finiteness properties, duality and many other properties listed in $\S 2.1$ also hold for them.

As emphasized before, the action of arithmetic subgroups on symmetric spaces has played an important role in understanding arithemetic subgroups. For $S$-arithmetic subgroups, symmetric spaces are replaced by products of symmetric spaces and Bruhat-Tits buildings.

Since the natural models of $\underline{E} \Gamma$ of $S$-arithmetic subgroups $\Gamma$ are products of symmetric spaces and Bruhat-Tits buildings and hence are not manifolds, there are no natural Riemannian manifolds associated with $S$-arithmetic subgroups as locally symmetric spaces associated with arithmetic subgroups. There is no analogue of spectral theory of locally symmetric spaces either, though the notion of automorphic representations still makes sense or one can try to combine the usual Laplacian operator for symmetric spaces and the discrete Laplacian for Bruhat-Tits buildings.

Remark 4.17. $\mathbb{Q}_{p}$ is an important example of a local compact field arising from the completion of a global field $\mathbb{Q}$. Another important example of global 
field is the function field of an algebraic curve over a finite field, for example $F_{p}(t)$, where $F_{p}$ is a finite field with $p$ elements, and $t$ is a variable. We can also define linear algebraic groups $\mathbf{G}$ over $F_{p}(t)$ and $S$-arithmetic subgroups for any finite set of places $S$ of the global field $F_{p}(t)$. Unlike the case of $S$ arithmetic subgroups of linear algebraic groups over $\mathbb{Q}, S$-arithmetic subgroups of $\mathbf{G}\left(F_{p}(t)\right)$ usually do not have many finiteness properties. For example, if the rank of $\mathbf{G}$ over $F_{p}(t)$ is positive, then $S$-arithmetic subgroups of $\mathbf{G}\left(F_{p}(t)\right)$ are not virtually torsion-free and do not admit a cofinite $\underline{E} \Gamma$-spaces. In fact, $S$ arithmetic subgroups of $\mathbf{G}\left(F_{p}(t)\right)$ are not even $F P_{\infty}$. On the other hand, if the rank of $\mathbf{G}$ over $F_{p}(t)$ is zero, $S$-arithmetic subgroups of $\mathbf{G}\left(F_{p}(t)\right)$ are virtually torsion-free and admit cofinite model of $E \Gamma$-spaces. Many other properties listed in $\$ 2.1$ hold for them too. See [199] for references.

\subsection{Generalizations of arithmetic groups: Non-lattice discrete subgroups and Patterson-Sullivan theory}

As discussed before, arithmetic subgroups of linear algebraic groups provide natural examples of discrete subgroups of Lie groups that are lattices. On the other hand, there are many examples of discrete subgroups of Lie groups that are not lattices. For example, the subgroup $\Gamma$ of $\operatorname{SL}(2, \mathbb{R})$ generated by $\left(\begin{array}{ll}1 & 1 \\ 0 & 1\end{array}\right)$ is a discrete subgroup but the volume of $\Gamma \backslash \mathrm{SL}(2, \mathbb{R})$ is not finite (or rather the area of the hyperbolic surface $\Gamma \backslash \mathbf{H}^{2}$ is not finite). Recall that a Fuchsian group is a discrete subgroup of $\operatorname{SL}(2, \mathbb{R})$, and it is said to be of the first kind if its limit set $\Lambda(\Gamma)$ in $\mathbf{H}^{2}(\infty)$ is equal to the whole boundary $\mathbf{H}^{2}(\infty)$. Otherwise it is said to be of the second kind. For a finitely generated Fuchsian group, it is of the first kind if and only if it is a lattice subgroup of $\operatorname{SL}(2, \mathbb{R})$. The Fuchsian group $\Gamma$ constructed above is an elementary subgroup since its limit set $\Lambda(\Gamma)$ contains only one point. There are also many non-elementary Fuchsian groups $\Gamma$ of the second kind, i.e., $\Gamma \backslash \mathbf{H}^{2}$ has infinite area.

Recall that a Kleinian group is a discrete subgroup that acts isometrically on the real hyperbolic space $\mathbf{H}^{3}$ of dimension 3, i.e., a discrete subgroup of $\operatorname{PSL}(2, \mathbb{C})$ (or $\operatorname{SL}(2, \mathbb{C})$ for convenience). A Kleinian group is called elementary if its limit set in $\mathbf{H}^{3}(\infty)$ contains at most 2 points. One interesting way to obtain a non-elementary Kleinian group is to take a cocompact Fuchsian group $\Gamma \subset \mathrm{SL}(2, \mathbb{R})$. Then the inclusion $\Gamma \subset \mathrm{SL}(2, \mathbb{C})$ gives a discrete subgroup of $\operatorname{SL}(2, \mathbb{C})$ that it not a lattice, and hence the hyperbolic space $\Gamma \backslash \mathbf{H}^{3}$ has infinite volume. Its limit set in $\partial \mathbf{H}^{3}$ is a circle and hence it is not an elementary group. On the other hand, $\Gamma \backslash \mathbf{H}^{3}$ has finite topology.

In general, for the hyperbolic spaces $\mathbf{H}^{n}$, there is a large class of Kleinian groups that are geometrically finite, for example through combination theorems (see [283]). For a general simple Lie group of rank 1, we can also define 
geometrically finite discrete subgroups (see [86]). For these geometrically finite Kleinian groups, all the finiteness properties and cohomological properties for arithmetic groups listed in $\S 2.1$ hold.

There are also important features that are more interesting for discrete subgroups that are not lattices. One particularly interesting example is the Patterson-Sullivan theory concerning measures supported on the limit sets of Kleinian groups $\Gamma$.

Briefly, the theory says that for any discrete group $\Gamma$ acting on $\mathbf{H}^{n}$, there is a class of measures on the limit set of $\Gamma$ in the boundary at infinity of $\mathbf{H}^{n}$ which is determined by the distribution of points in each $\Gamma$-orbit, and these measures reflect the spectral properties and the ergodic theory of the geodesic flow of the quotient manifold $\Gamma \backslash \mathbf{H}^{n}$. The Hausdorff dimension of the limit set is related to the bottom of the spectrum of $\Gamma \backslash \mathbf{H}^{n}$. The bottom of the spectrum also has a positive eigenfunction. See the original papers [343] [397], and the book [330]. There are some generalizations to higher rank Lie groups and their discrete subgroups. See [5] [358] [259]. At one point, it seemed that one difficulty with the higher rank case was the lack of abundant examples of discrete subgroups that are not lattices and hence not too large, but not too small either. By the recent results in [250] and [87], the Hitchin representations of surface groups and maximal representations of surface groups for semisimple Lie groups of Hermitian type give classes of Zariski dense discrete subgroups of reductive Lie groups of higher rank that are not lattices.

\subsection{Symmetric spaces and actions of arithmetic groups}

Let $G$ be any Lie group with finitely many connected components, and $K \subset G$ be a maximal compact subgroup. Then the homogeneous space $X=G / K$ is diffeomorphic to $\mathbb{R}^{n}$, where $n=\operatorname{dim} X$. Any arithmetic subgroup $\Gamma$ of $G$ acts properly discontinuously on $X$.

If $G$ is a reductive Lie group, for example, $G=\operatorname{GL}(n, \mathbb{R})$, then $X$ with any invariant Riemannian metric is a symmetric space of nonpositive sectional curvature. If $G$ is semisimple, then $X$ is a symmetric space of noncompact type.

Recall that a Riemannian manifold $M$ is called a locally symmetric space if for every point $x \in M$, the locally defined geodesic symmetry that reverses every geodesic passing though $x$ is a local isometry. A Riemannian manifold $X$ is called a symmetric space if it is locally symmetric, and for every point $x \in X$, the local geodesic symmetry extends to a global isometry.

We note that a symmetric space is automatically complete. On the other hand, locally symmetric spaces are not necessarily complete. For example, if $X$ is a symmetric space, then for any point $p \in X$, the complement $X-\{p\}$ is a locally symmetric space. 
It is known that if $X$ is a symmetric space, then the identity component of its isometry group $\operatorname{Isom}^{0}(X)$, denoted by $G$, acts transitively on $X$, hence $X$ can be identified with $G / K$, where $K$ is the stabilizer of any point in $X$. It is also known that we can always replace $G$ by a reductive Lie group. (Note the isometry group of $\mathbb{R}^{n}$ is not reductive and this is the only exception among symmetric spaces.)

It is known that the universal covering of a complete locally symmetric space is a symmetric space. This implies that any complete locally symmetric space can be written in the form $\Gamma \backslash G / K$, where $G$ is a reductive Lie group, $K$ is a proper maximal compact subgroup of $G$, and $\Gamma \subset G$ is a discrete subgroup.

According to the definition, a locally symmetric space should be a smooth manifold. Since many natural arithmetic groups contain torsion elements, the quotient spaces $\Gamma \backslash X$ are often not smooth manifolds, but rather orbifolds. In view of this, whenever $G$ is reductive, for any discrete subgroup $\Gamma \subset G, \Gamma \backslash X$ is usually called a locally symmetric space as well. Of course, the most interesting class of locally symmetric spaces consists of locally symmetric spaces of finite volume.

It is also known that locally symmetric spaces are characterized by the condition that the curvature tensor is parallel, i.e., the covariant derivative of the curvature tensor is zero. This immediately implies that if $M$ is a Riemannian manifold of constant sectional curvature, then it is a locally symmetric space. In particular, hyperbolic manifolds are locally symmetric spaces.

It is also known that a simply connected symmetric space $X$ can be uniquely written as a product $\mathbb{R}^{n} \times X_{1} \times \cdots \times X_{m}$, where each $X_{i}$ is irreducible in the sense whenever $X_{i}$ is not a product of two Riemannian manifolds, or equivalently if $X_{i}$ is written as $G_{i} / K_{i}$, where $G_{i}$ is the identity component of the isometry group of $X_{i}$, then the associated involutive Lie algebra or the pair $\left(\mathfrak{g}_{i}, \mathfrak{k}_{i}\right)$ is irreducible. The factor $\mathbb{R}^{n}$ is called the flat factor of $X$.

We note that in the above decomposition, the assumption that $X$ is simply connected is important. For example, for any lattice $\Lambda \subset \mathbb{R}^{n}$, the quotient $\Lambda \backslash \mathbb{R}^{n}$ is a symmetric space. If $\Lambda$ is irreducible, i.e., there is no isometric splitting $\mathbb{R}^{n}=\mathbb{R}^{n_{1}} \times \mathbb{R}^{n_{2}}$ such that $\Lambda=\left(\Lambda \cap \mathbb{R}^{n_{1}} \times\{0\}\right) \times\left(\Lambda \cap\{0\} \times \mathbb{R}^{n_{2}}\right)$, then $\Lambda \backslash \mathbb{R}^{n}$ is not isometric to a product, though $\mathbb{R}^{n}$ is reducible.

The Euclidean space $\mathbb{R}^{n}$ is a flat symmetric space. A nonflat irreducible symmetric space $X$ has either nonpositive sectional curvature or nonnegative sectional curvature. If the sectional curvature of $X$ is nonpositive, $X$ is called of noncompact type, and otherwise it is called of compact type. The two important examples are the real hyperbolic space $\mathbf{H}^{n}$ and the unit sphere $S^{n}$ in $\mathbb{R}^{n+1}$.

A symmetric space is called of compact type if it is simply connected, does not contain a nontrivial flat factor $\mathbb{R}^{n}$, and if its irreducible factors are of compact type. Symmetric spaces of noncompact type can be defined similarly.

A very important notion concerning the geometry of symmetric spaces is the notion of rank. A flat subspace of dimension $r$ of a symmetric space $X$ is 
an isometric immersion $\mathbb{R}^{r} \rightarrow X$. When $X$ is of compact type, the image is compact, isometric to a flat torus. If $X$ is of noncompact type, then $\mathbb{R}^{r} \rightarrow X$ is an isometric embedding. The maximal dimension of flat subspaces of $X$ is called the rank of $X$. The real hyperbolic space $\mathbf{H}^{n}$ is of rank 1 , and the symmetric space $\mathrm{SL}(n, \mathbb{R}) / \mathrm{SO}(n)$ is of rank $n-1$. The rank is additive in the sense that the rank of the product $X_{1} \times X_{2}$ is the sum of the ranks of $X_{1}$ and $X_{2}$.

If $G=\mathbf{G}(\mathbb{R})$ is the real locus of a linear algebraic group $\mathbf{G}$ defined over $\mathbb{R}$, then the rank of $X$ is equal to the $\mathbb{R}$-rank of $\mathbf{G}$, i.e., the maximal dimension of $\mathbb{R}$-split tori contained in $\mathbf{G}$. In fact, maximal flats in $X$ are orbits of the real locus of such maximal split tori in $\mathbf{G}$.

The volume of a ball of radius $R$ in a symmetric space $X$ of noncompact type grows exponentially in $R$. In fact, let $\mathfrak{g}=\mathfrak{k}+\mathfrak{p}$ be the Cartan decomposition of the Lie algebra $\mathfrak{g}$ of $G$, and $\mathfrak{a} \subset \mathfrak{p}$ be a maximal abelian subalgebra. Then we have a root space decomposition of $\mathfrak{g}$ :

$$
\mathfrak{g}=\mathfrak{g}_{0}+\sum_{\alpha \in \Sigma(\mathfrak{g}, \mathfrak{a})} \mathfrak{g}_{\alpha}
$$

Choose a positive chamber of $\mathfrak{a}$ and hence a set of positive roots $\Sigma^{+}(\mathfrak{g}, \mathfrak{a})$. Let $\rho$ be the half sum of positive roots with multiplicity given by $\operatorname{dim} \mathfrak{g}_{\alpha}$. The Killing form of $\mathfrak{g}$ induces an invariant Riemannian metric on the symmetric space $X=G / K$. Let $x_{0}$ be the basepoint of $X$ corresponding to the identity coset $K \subset G$. Let $B\left(x_{0}, R\right)$ be the ball of radius $R$ with center at $x_{0}$. Then it is well-known that

$$
\lim _{R \rightarrow+\infty} \frac{\log \operatorname{vol}\left(B\left(x_{0}, R\right)\right)}{R}=2\|\rho\| .
$$

More precise information is known [240, Theorem $\mathrm{A}$, and $\S 6]$ : as $R \rightarrow+\infty$,

$$
\operatorname{vol}\left(B\left(x_{0}, R\right)\right) \sim R^{\frac{r-1}{2}} e^{2\|\rho\| R},
$$

where $r$ is the rank of $X$.

For studying topological properties of arithmetic groups, the following result is important.

Proposition 4.18 (Cartan fixed point theorem). Assume that $G$ is semisimple, and $X$ is a symmetric space of noncompact type. Then for any compact subgroup $C$ of $G$, the set of fixed points of $C$ in $X$ is a nonempty totally geodesic submanifold.

Proof. Since $X$ is a simply connected and nonpositively curved Riemannian manifold, every compact subgroup $C$ of $G$ has at least one fixed point in $X$. In fact, for any point $x \in X$, the center of gravity of the orbit $C \cdot x$ exists and is fixed by $C$. Since $C$ acts by isometries on $X$, its set of fixed points is a totally geodesic submanifold. 


\subsection{Fundamental domains and generalizations}

Suppose that a discrete group $\Gamma$ acts properly discontinuously on a topological space $X$. An effective way to understand the quotient $\Gamma \backslash X$ and properties of $\Gamma$ is to find good fundamental domains for the $\Gamma$-action on $X$. For example, suppose that $X$ is a measure space and the $\Gamma$-action preserves the measure. It is naturally expected that the measure descends to a measure on the quotient. It turns out that it can be defined using a measurable fundamental set (see the discussion after Proposition 4.20).

Since there have been many different notions of fundamental sets, fundamental domains and fundamental regions, we will recall several definitions in order to clarify their meanings.

Probably the most obvious definition of a fundamental set for a $\Gamma$-action on $X$ is a subset of $X$ that meets every $\Gamma$-orbit once. Its existence follows from the axiom of choice. In general, we impose some additional structures on fundamental sets so that they can be used to understand the quotient $\Gamma \backslash X$ as a topological space or with another more refined structure. Due to the conventional meaning of fundamental sets in the reduction theory of arithmetic groups, we reserve the name fundamental set for something else in dealing with actions of arithmetic groups.

Since $X$ is a topological space and $\Gamma$ acts by homeomorphisms, a natural notion is that of fundamental domains. Recall that an open subset of $X$ is called a fundamental domain of the $\Gamma$-action on $X$ if the following conditions are satisfied:

(1) The $\Gamma$-translates of the closure $\bar{\Omega}$ cover $X, X=\cup_{\gamma \in \Gamma} \gamma \bar{\Omega}$,

(2) The $\Gamma$-translates of $\Omega$ are disjoint, and hence the map $\Omega \rightarrow \Gamma \backslash X$ is injective.

(3) The boundary $\partial \Omega$ is small in a certain sense, for example, the interior of $\bar{\Omega}$ is equal to $\Omega$. If $X$ is a measure space, it is natural to impose that the boundary $\partial \Omega$ has measure 0 , so that we expect that $\Omega, \bar{\Omega}$ and $\Gamma \backslash X$ have the same total measure.

Since it is sometimes more convenient to describe the closure $\bar{\Omega}$, we will also call $\bar{\Omega}$, or even some subsets between $\Omega$ and $\bar{\Omega}$, a fundamental domain for the $\Gamma$-action.

Remark 4.19. If $X$ is a smooth manifold, and $\Omega$ is the interior of a submanifold with corners, then the conditions in (3) are certainly satisfied. But for general spaces $X$ and $\Gamma$-actions, fundamental domains have more complicated structures. Usually we require the boundary of $\Omega$ to be not too complicated and small in some sense. 
But in general, the existence of such a fundamental domain in $X$ is not clear. As shown below, if $X$ is a Riemannian manifold and $\Gamma$ acts isometrically on $X$, then there always exist such fundamental domains (Proposition 4.22).

If $X$ is a measure space and the $\Gamma$-action is measure preserving, then it is more natural to require that fundamental sets be measurable subsets, for example, Borel sets.

Proposition 4.20. Let $X$ be a second countable topological space, and let $\mu$ be a measure on $X$ which is preserved by the $\Gamma$-action. Then there exists a Borel subset $F$ of $X$ that meets every $\Gamma$-orbit exactly once.

When $X$ is taken to be a topological group $G$, in [390], if a subset $F$ of $G$ satisfies the conditions:

(1) $\Gamma F=G$,

(2) $F$ meets every $\Gamma$-orbit exactly once, i.e., for two different elements $\gamma_{1}, \gamma_{2} \in$ $\Gamma, \gamma_{1} F \cap \gamma_{2} F=\emptyset$,

(3) $F$ is a Borel set,

then $F$ is called a fundamental set of the subgroup $\Gamma$. If $G$ is second countable, then such fundamental sets were constructed in [390, Lemma 2]. The same proof works in the above more general situation.

Once we have constructed such a measurable fundamental set $F$, we can define a measure on $\Gamma \backslash X$ as follows. Let $\pi: X \rightarrow \Gamma \backslash X$ be the projection. Then a subset $S \subset \Gamma \backslash X$ is defined to be measurable if $\pi^{-1}(S) \cap F$ is measurable, and we define

$$
\mu(S)=\mu\left(\pi^{-1}(S) \cap F\right) .
$$

It can be shown that this definition of the measure on $\Gamma \backslash X$ is independent of the choice of $F$.

It is often convenient and important to impose some finiteness conditions on fundamental domains. One such condition is local finiteness: for any compact subset $C \subset X$, the set $\{\gamma \in \Gamma \mid \gamma \bar{\Omega} \cap C \neq \emptyset\}$ is finite, i.e., any compact subset $C$ is covered by only finitely many translates of $\bar{\Omega}$. In [390], fundamental sets satisfying this local finiteness are called normal fundamental sets.

The $\Gamma$-action on $X$ induces an equivalence relation on $X$, and it induces an equivalence relation on the closure $\bar{\Omega}$. Denote the quotient by $\bar{\Omega} / \sim$. Denote the projection map $X \longrightarrow \Gamma \backslash X$ by $\pi$. Its restriction to $\bar{\Omega}$ defines a map $\bar{\Omega} / \sim \longrightarrow \Gamma \backslash X$, also denoted by $\pi$.

Proposition 4.21. Assume that $\Omega$ is a locally finite fundamental domain for the $\Gamma$-action on $X$. Then the map $\pi: \bar{\Omega} / \sim \longrightarrow \Gamma \backslash X$ is a homeomorphism.

See [29, Theorem 9.2.4] for a proof. This proposition says that up to homeomorphism, $\Gamma \backslash X$ can be obtained from the closure $\bar{\Omega}$ by identifying suitable 
points on the boundary. This is one instance where a fundamental domain can be used to understand the quotient $\Gamma \backslash X$ as a topological space, i.e., by identifying some points on the boundary of $\Omega$. The best example to illustrate this result is to consider the action $\Gamma=\mathbb{Z}^{2}$ on $\mathbb{R}^{2}$ by translation. Then the open unit square is a fundamental domain, and the quotient $\mathbb{Z}^{2} \backslash \mathbb{R}^{2}$ is obtained by identifying points on the boundary intervals of the unit square to get a torus. Another good example is to take the standard fundamental domain for the $\mathrm{SL}(2, \mathbb{Z})$-action on the upper half plane $\mathbf{H}^{2}$. Identifying the sides, we can show that $\mathrm{SL}(2, \mathbb{Z}) \backslash \mathbf{H}^{2}$ is homeomorphic to $\mathbb{C}$.

Another important finiteness condition is the global finiteness condition: $\{\gamma \in \Gamma \mid \gamma \bar{\Omega} \cap \bar{\Omega} \neq \emptyset\}$ is finite, i.e, each translate of $\bar{\Omega}$ meets only finitely many other translates by elements of $\Gamma$, and the overlap on the boundary of these $\Gamma$-translates is uniformly bounded. The importance of a fundamental domain satisfying the global finiteness condition is that its existence implies $\Gamma$ is finitely generated (see Proposition 4.39 below, and [390] [29, Theorem 9.2.7] or [348]). This probably explains why it is not obvious that fundamental domains satisfying global or local finiteness conditions should exist for a general proper action of a discrete group.

Rough (or coarse) fundamental domains.

It is often difficult to find or construct fundamental domains. A subset $\mathcal{R}$ of $X$ is called a rough (or coarse) fundamental domain for the $\Gamma$-action on $X$ if the following conditions are satisfied:

(1) The $\Gamma$-translates of $\overline{\mathcal{R}}$ cover $X$, i.e., $\overline{\mathcal{R}}$ meets every $\Gamma$-orbit.

(2) $\mathcal{R}$ meets every $\Gamma$-orbit at most finitely many times.

In this case, we usually do not impose conditions on the boundary of $\mathcal{R}$, though many examples in applications do have small boundaries in some sense, for example, we often take $\mathcal{R}$ to be an open subset, and the interior of the closure $\overline{\mathcal{R}}$ is equal to $\mathcal{R}$.

It is often easier to construct and describe rough fundamental domains than fundamental domains, and their structures at infinity are simpler in general. Picking out a fundamental domain inside a rough fundamental domain might be complicated. When a symmetric space $X=G / K$ is not a hyperbolic space, the action of arithmetic subgroups of $G$ on $X$ provides such examples. But for some applications, rough fundamental domains satisfying suitable conditions are sufficient.

From the above definitions, it is clear that a fundamental domain is a rough fundamental domain.

Usually there are some finiteness conditions imposed on rough fundamental domains as well. The local finiteness is satisfied by many known fundamental domains. But we often impose the stronger global finiteness condition requiring that the subset $\{\gamma \in \Gamma \mid \gamma \overline{\mathcal{R}} \cap \overline{\mathcal{R}} \neq \emptyset\}$ is finite, i.e., each translate of $\overline{\mathcal{R}}$ meets 
only finitely many other translates, which implies that the map $\overline{\mathcal{R}} \rightarrow \Gamma \backslash X$ is uniformly finite-to-one. This condition is important in combinatorial properties of $\Gamma$ and its existence implies that $\Gamma$ is finitely generated (See Proposition 4.39).

Rough fundamental domains constructed in the reduction theory of arithmetic groups acting on symmetric spaces satisfy such a global finiteness condition. The global finiteness condition is usually called the Siegel finiteness condition, and the rough fundamental domains are usually called fundamental sets in [57] and in literature on arithmetic groups and automorphic forms. We should emphasize that this is not the fundamental set defined at the beginning of this subsection and in other places such as [29] and [390].

\section{Dirichlet fundamental domains.}

If $X$ is a proper and complete metric space and $\Gamma$ acts isometrically and properly discontinuously, then a convenient way to obtain a fundamental domain is to take the Dirichlet fundamental domain.

Suppose that there exist points in $X$ that are not fixed by any nontrivial element of $\Gamma$. For any basepoint $x_{0} \in X$ not fixed by any nontrivial element of $\Gamma$, define

$$
\bar{D}\left(x_{0}, \Gamma\right)=\left\{x \in X \mid d\left(x, x_{0}\right) \leq d\left(\gamma x, x_{0}\right) \text { for all } \gamma \in \Gamma\right\} .
$$

Assume that $X$ is locally compact. Then every $\Gamma$-orbit meets $\bar{D}\left(x_{0}, \Gamma\right)$ at least once. One way to see this is as follows: in each $\Gamma$-orbit, pick the set of points of minimal distance from $x_{0}$. Since $\Gamma$ acts properly discontinuously on $X$ and $X$ is a proper metric space, such points exist. The union of such points of minimal distance to $x_{0}$ is equal to $\bar{D}\left(x_{0}, \Gamma\right)$.

Replacing the non-strict inequalities by strict inequalities, we obtain a domain

$$
D\left(x_{0}, \Gamma\right)=\left\{x \in X \mid d\left(x, x_{0}\right)<d\left(\gamma x, x_{0}\right) \text { for all } \gamma \in \Gamma\right\} .
$$

This is usually called the Dirichlet domain of $\Gamma$ with center at $x_{0}$.

It is natural to guess that the closure of $D\left(x_{0}, \Gamma\right)$ is equal to $\bar{D}\left(x_{0}, \Gamma\right)$ (or the interior of $\bar{D}\left(x_{0}, \Gamma\right)$ is equal to $\left.D\left(x_{0}, \Gamma\right)\right)$ and is a fundamental domain for the $\Gamma$-action. But this is not true for general metric spaces. The counterexample in [328] explores the following non-intuitive fact: there exists a metric space $(X, d)$ such that for two different points $p_{1}, p_{2}$, the bisector $\left\{x \in X \mid d\left(x, p_{1}\right)=\right.$ $\left.d\left(x, p_{2}\right)\right\}$ contains open subsets of $X$. For example, take $X=\mathbb{R}^{2}$ with the $L^{1}$ metric,

$$
d\left((x, y),\left(x^{\prime}, y^{\prime}\right)\right)=\left|x-x^{\prime}\right|+\left|y-y^{\prime}\right|,
$$

and the points $p_{1}=(-1,-1), p_{2}=(1,1)$. Then the bisector contains both the second and fourth quadrants of the plane $\mathbb{R}^{2}$. 
Proposition 4.22. Assume that $X$ is a complete Riemannian manifold or a Euclidean simplicial complex (i.e., its metric restricts to the standard Euclidean metric on each simplex) and is complete. Then the following results hold:

(1) The bisector of every pair of different points is a subset of $X$ of codimension 1.

(2) The closure of $D\left(x_{0}, \Gamma\right)$ is equal to $\bar{D}\left(x_{0}, \Gamma\right)$.

(3) $D\left(x_{0}, \Gamma\right)$ is a locally finite fundamental domain for the $\Gamma$-action on $X$.

In particular, the $\Gamma$-action admits a fundamental domain in the sense defined above.

(1) can be proved by contradiction and the fact that any minimizing geodesic segment connecting two points is smooth, and (2) follows from (1). (3) follows from the proof of a similar result in [29].

Under the above condition, $D\left(x_{0}, \Gamma\right)$ is called the Dirichlet fundamental domain of the $\Gamma$-action with center $x_{0}$. Sometimes, we also call its closure $\bar{D}\left(x_{0}, \Gamma\right)$ a Dirichlet fundamental domain for the $\Gamma$-action as well.

Recall that the property that a simply connected complete Riemannian manifold $X$ has no conjugate point means that every pair of distinct points of $X$ are joined by a unique geodesic segment up to parametrization. This condition is satisfied if $X$ is a Hadamard manifold, i.e., a simply connected complete Riemannian manifold of nonpositive sectional curvature. If $X$ is a simply connected complete Riemannian manifold without conjugate points, more structure of the boundary of the Dirichlet fundamental domain is known. See [117].

\subsection{Fundamental domains for Fuchsian groups and applications to compactification}

Though the Dirichlet fundamental domain for any $\Gamma$-action on $X$ is canonically defined once the center $x_{0}$ is fixed, it is usually useful only for special spaces $X$. For example, when $X$ is the Euclidean space $\mathbb{R}^{n}$, Dirichlet introduced this notion for lattices $\Lambda \subset \mathbb{R}^{n}$ in 1850. It is closely related to the more general Voronoi cells. Later Poincaré generalized the notion of Dirichlet fundamental domains to discrete isometric actions on hyperbolic spaces.

When $X$ is the hyperbolic plane $\mathbf{H}^{2}$ and $\Gamma$ is finitely generated, every Dirichlet fundamental domain $D\left(x_{0}, \Gamma\right)$ is bounded by finitely many geodesics. In particular, $D\left(x_{0}, \Gamma\right)$ satisfies both the local and global finiteness properties mentioned in the previous subsection.

Dirichlet fundamental domains have played an important role in the study of Fuchsian groups $\Gamma$. For example, assume that $\Gamma$ is a lattice. Then it is known 
that any Dirichlet fundamental domain $D\left(x_{0}, \Gamma\right)$ is bounded by finitely many geodesic sides and hence $\Gamma$ is finitely generated [391]. The Poincaré upper half plane $\mathbf{H}^{2}$ admits a natural compactification by adding the boundary circle $\mathbf{H}^{2}(\infty)=\mathbb{R} \cup\{\infty\}$. The limit points of $D\left(x_{0}, \Gamma\right)$ in the boundary circle are called cusp points of $D\left(x_{0}, \Gamma\right)$. If $\Gamma$ is a non-uniform lattice, or a Fuchsian group of the first kind, then $D\left(x_{0}, \Gamma\right)$ has finitely many cusp points at infinity and they correspond to parabolic subgroups of $\Gamma$ defined below (or $\Gamma$-rational parabolic subgroups of $\operatorname{SL}(2, \mathbb{R})$ ). (Note that the cusps of the quotient $\Gamma \backslash \mathbf{H}^{2}$ correspond to $\Gamma$-conjugacy classes of parabolic subgroups of $\Gamma$, but some cusp points of $D\left(x_{0}, \Gamma\right)$ may be projected to the same cusp of $\Gamma \backslash \mathbf{H}^{2}$.)

We note that the $\Gamma$-action on $\mathbf{H}^{2}$ extends to the compactification $\mathbf{H}^{2} \cup$ $\mathbf{H}^{2}(\infty)$. We call a point in $\mathbf{H}^{2}(\infty)$ a $\Gamma$-rational boundary (or cusp) point if it is $\Gamma$-equivalent to a cusp of $D\left(x_{0}, \Gamma\right)$.

Define a subgroup of $\Gamma$ to be a parabolic subgroup if it is the stabilizer of a $\Gamma$-cusp point. Then it can be shown that each parabolic subgroup consists of only parabolic elements, and every parabolic element is contained in some parabolic subgroup of $\Gamma$. Since $D\left(x_{0}, \Gamma\right)$ has only finitely many cusp points, it follows that $\Gamma$ contains finitely many conjugacy classes of parabolic subgroups, and parabolic elements of $\Gamma$ are conjugate to elements that fix some cusps of $D\left(x_{0}, \Gamma\right)$.

The above notion of parabolic subgroups of $\Gamma$ is from the theory of Fuchsian groups. According to the general definition from the theory of Lie groups and algebraic groups, a closed subgroup $P$ of $\mathrm{SL}(2, \mathbb{R})$ is called a parabolic subgroup if and only if the quotient $P \backslash \mathrm{SL}(2, \mathbb{R})$ is compact. It can be proved that a subgroup of $\operatorname{SL}(2, \mathbb{R})$ is a parabolic subgroup if and only if it fixes a boundary point in $\mathbf{H}^{2}(\infty)$. We call a parabolic subgroup of $\operatorname{SL}(2, \mathbb{R}) \Gamma$-rational if it fixes a $\Gamma$-cusp point. Then the following result holds and clarifies the relation between two definitions of parabolic subgroups.

Proposition 4.23. For any $\Gamma$-rational parabolic subgroup $P$ of $\operatorname{SL}(2, \mathbb{R})$, the intersection $P \cap \Gamma$ is a parabolic subgroup of $\Gamma$, and every parabolic subgroup of $\Gamma$ is of this form.

Another characterizaion of $\Gamma$-parabolic subgroups is the following one.

Proposition 4.24. A parabolic subgroup $P$ of $\mathrm{SL}(2, \mathbb{R})$ is $\Gamma$-rational if and only if the intersection $P \cap \Gamma$ is a lattice of the unipotent radical $N_{P}$ of $P$, which is equivalent in this case to the condition that $P \cap \Gamma$ is an infinite subgroup.

When $P$ is the parabolic subgroup consisting of upper triangular matrices, then $N_{P}$ is the subgroup consisting of upper triangular matrices with $1 \mathrm{~s}$ on the diagonal. One consequence of this result is the following.

Proposition 4.25. There is a one-to-one correspondence between the set of conjugacy classes of $\Gamma$-rational parabolic subgroups of $\mathrm{SL}(2, \mathbb{R})$ (or equivalently 
the set of conjugacy classes of parabolic subgroups of $\Gamma$ ) and the set of ends of $\Gamma \backslash \mathbf{H}^{2}$.

These results on relations between $\Gamma$-parabolic subgroups and $\Gamma$-cusp points can be used to construct compactifications of $\Gamma \backslash \mathbf{H}^{2}$. For example, by adding all $\Gamma$-cusps to $\mathbf{H}^{2}$, we get an enlarged space that lies between $\mathbf{H}^{2}$ and $\mathbf{H}^{2} \cup \mathbf{H}^{2}(\infty)$. Naturally, it has the subspace topology induced from the compactification of $\mathbf{H}^{2}$. By strengthening this induced subset topology so that for every cusp point, it has a neighborhood basis, each of which is stabilized by the corresponding parabolic subgroup of $\Gamma$, we obtain a partial compactification $\overline{\mathbf{H}}^{2} S$. The strengthened topology is called the Satake topology.

It is perhaps helpful to point out that the induced subspace topology does not contain any neighborhood basis of a cusp point that is stable under the stabilizer of the cusp. In fact, if we start with any neighborhood $U$ of the cusp in the compactification $\mathbf{H}^{2} \cup \mathbf{H}^{2}(\infty)$, then for $\gamma$ in the stabilizer of the cusp, the translates $\gamma U$ cover the compactification $\mathbf{H}^{2} \cup \mathbf{H}^{2}(\infty)$, and the translates $\gamma U \cap \mathbf{H}^{2}$ cover the whole space $\mathbf{H}^{2}$.

Using the Satake topology, it can be proved that $\Gamma$ acts continuously on the partial compactification $\overline{\mathbf{H}}^{2} S$ with a compact, Hausdorff quotient $\Gamma \backslash \overline{\mathbf{H}}^{2} S$.

The compactification $\Gamma \backslash \overline{\mathbf{H}}^{2}$ is obtained from $\Gamma \backslash \mathbf{H}^{2}$ by adding one point to every end (or cusp neighborhood). This is the simplest example of Satake compactifications of locally symmetric spaces and also of the Baily-Borel compactification of Hermitian locally symmetric spaces. See $\S 4.17$ for the general case.

The same procedure can be applied to construct the Borel-Serre compactification of $\Gamma \backslash \mathbf{H}^{2}$. In the partial compactification $\overline{\mathbf{H}}^{2}$, blow up every cusp point to $\mathbb{R}$, which is equal to $N_{P}$, where $P$ is the corresponding $\Gamma$-rational parabolic subgroup and $N_{P}$ is the unipotent radical of $P$. The resulting space is the Borel-Serre partial compactification $\overline{\mathbf{H}}^{2}$. . It is a real analytic manifold with boundary and $\Gamma$ acts on it real analytically and properly. The quotient $\Gamma \backslash \overline{\mathbf{H}}^{2}{ }^{B S}$ is a compact manifold with boundary. It is mapped surjectively to the Satake compactification $\Gamma \backslash{\overline{\mathbf{H}^{2}}}^{S}$, and the inverse image of every boundary point of $\Gamma \backslash{\overline{\mathbf{H}^{2}}}^{S}$ is equal to a circle. See $\S 4.18$ for the general case.

The difference between these two compactifications is that $\Gamma \backslash \overline{\mathbf{H}}^{2} S$ admits a complex structure as a compact Riemann surface, while $\Gamma \backslash \overline{\mathbf{H}}^{2}{ }^{B S}$ is a manifold with boundary. Furthermore, the inclusion $\Gamma \backslash \mathbf{H}^{2} \rightarrow \Gamma \backslash \overline{\mathbf{H}}^{2}$ is not a homotopy equivalence since the loops around the cusps are homotopically trivial in $\Gamma \backslash \overline{\mathbf{H}}^{2}$, but the inclusion $\Gamma \backslash \mathbf{H}^{2} \rightarrow \Gamma \backslash \overline{\mathbf{H}}^{2}{ }^{B S}$ is a homotopy equivalence. 
When $\Gamma$ is torsion-free, $\Gamma \backslash \overline{\mathbf{H}}^{2}$ is a finite model of $B \Gamma$-space. If $\Gamma$ contains torsion elements, then the Borel-Serre partial compactification $\overline{\mathbf{H}}^{B S}$ is a cofinite model of $\underline{E} \Gamma$.

For other spaces, for example, symmetric spaces $X=G / K$ that are not real hyperbolic spaces and of higher rank, there is no such nice structure of the Dirichlet fundamental domains as above. For example, $D\left(x_{0}, \Gamma\right)$ is not bounded by totally geodesic hypersurfaces. If $\Gamma \backslash X$ is noncompact, the notion of cusps is not defined to satisfy the above simple and clean relation with $\mathbb{Q}$-parabolic subgroups of $\mathbf{G}$, and the structure near infinity of $D\left(x_{0}, \Gamma\right)$ is often complicated and not adapted to parabolic subgroups of $G$. Therefore, Dirichlet fundamental domains are not suitable for understanding analysis, geometry and compactifications of $\Gamma \backslash X$.

In some sense, the reduction theory of arithmetic subgroups is about finding suitable fundamental domains or rough fundamental domains for actions of arithmetic subgroups $\Gamma$ on symmetric spaces that reflect structures of $\Gamma$ and $G$ as in the case of Dirichlet fundamental domains for actions of Fuchsian groups on $\mathbf{H}^{2}$. It turns out that fundamental sets (or rough fundamental domains) defined in terms of Siegel sets of parabolic subgroups serve such purposes well and hence are used in the reduction theory of arithmetic subgroups [57].

One major application of the reduction theory of arithmetic subgroups is to construct compactifications of $\Gamma \backslash X$ similar to $\Gamma \backslash{\overline{\mathbf{H}^{2}}}^{S}$ and $\Gamma \backslash{\overline{\mathbf{H}^{2}}}^{B S}$. For example, they allow us to pick out "rational boundary points".

In the above discussion, we started with a Fuchsian group and obtained Dirichlet fundamental domains and used them to study the quotient space $\Gamma \backslash \mathbf{H}^{2}$ and parabolic subgroups of $\Gamma$. Dirichlet fundamental domains are also useful in describing combinatorial properties of Fuchsian groups $\Gamma$. In fact, there are elements of $\Gamma$ that pair geodesic sides of $D\left(x_{0}, \Gamma\right)$. These elements generate $\Gamma$ and relations between them can also be read off from their actions on the sides of $D\left(x_{0}, \Gamma\right)$.

An important feature of Fuchsian groups is that we can reverse this process and construct Fuchsian groups from suitable hyperbolic polygons by giving generators and relations. This is called the Poincaré polygon theorem. There is also a higher dimensional generalization which replaces polygons by polyhedra. Probably the best examples are given by the Hecke triangle groups [400]. There is also the Klein combination theorem for Klein groups. The Klein combination theorem also works for groups acting on hyperbolic spaces in higher dimensions. See [29, Theorem 9.8.4] and [283].

It is perhaps worthwhile to point out that there is no analogue of the the Poincaré polyhedron theorem or the Klein combination theorem for other symmetric spaces. 


\subsection{Minkowski reduction theory for $\operatorname{SL}(n, \mathbb{Z})$}

As discussed in the previous subsection, good fundamental domains for Fuchsian groups have played an important role in understanding the structure of Riemann surfaces and their compactifications, and also algebraic structures of Fuchsian groups.

For arithmetic groups, the original motivation for reduction theory was slightly different. It was started by Lagrange and Gauss.

Note that the Poincaré upper half plane $\mathbf{H}^{2}=\mathrm{SL}(2, \mathbb{R}) / \mathrm{SO}(2)$ can be identified with the space of binary positive definite quadratic forms of determinant 1 , since $\operatorname{SL}(2, \mathbb{R})$ also acts transitively on the latter, with the stabilizer of the quadratic form $x^{2}+y^{2}$ equal to $\mathrm{SO}(2)$. The quotient $\mathrm{SL}(2, \mathbb{Z}) \backslash \mathbf{H}^{2}$ can be identified with the equivalence classes of such quadratic forms, where two quadratic forms $Q_{1}(x, y), Q_{2}(x, y)$ are defined to be equivalent if they become equal under a change of variables by an element of $\mathrm{SL}(2, \mathbb{Z})$. Consequently, two equivalent quadratic forms represent the same set of values over the integers.

An important problem is to find "good" representatives in each equivalence class, and the notion of reduced quadratic form was introduced by Lagrange and Gauss. These representatives correspond to points of the usual fundamental domain $\left\{z \in \mathbf{H}^{2}|| z|\geq 1,| \operatorname{Re}(z) \mid \leq \frac{1}{2}\right\}$ for the $\operatorname{SL}(2, \mathbb{Z})$-action on $\mathbf{H}^{2}$.

After that, the problem of finding fundamental domains (or rough fundamental domains) for arithmetic subgroups was called reduction theory.

For $n \geq 3$, a reduction theory for $\Gamma=\operatorname{SL}(n, \mathbb{Z})$ acting on $\operatorname{SL}(n, \mathbb{R}) / \mathrm{SO}(n)$ was developed by Minkowski. Since this motivates directly a generalization for the action of the mapping class group $\operatorname{Mod}_{g}$ on the Teichmüller space $\mathcal{T}_{g}$ which we discuss below in $\S 5.11$, we briefly recall its definition. For the original papers of Minkowski, see [305]. See also the books [392] [402] for more detail.

For various purposes, it will be easier to consider the reduction theory for the action of $\mathrm{SL}(n, \mathbb{Z})$ on $\mathrm{GL}^{+}(n, \mathbb{R}) / \mathrm{SO}(n)$, which can be identified with the space of positive quadratic forms in $n$-variables, denoted by $\mathcal{P}_{n}$. The subspace $\mathrm{SL}(n, \mathbb{R}) / \mathrm{SO}(n)$ is denoted by $S \mathcal{P}_{n}$. For each positive quadratic form $Q\left(x_{1}, \cdots, x_{n}\right)=\sum_{i, j=1}^{n} q_{i j} x_{i} x_{j}$, denote its associated symmetric matrix $\left(y_{i j}\right)$ by $Q$ as well.

Let $e_{1}, \cdots, e_{n}$ be the standard basis of $\mathbb{Z}^{n}$ as above. Define the Minkowski reduction domain by

$$
\begin{aligned}
& \mathcal{D}_{n}^{M}=\left\{Q \in \mathcal{P}_{n} \mid q_{i i} \leq Q(v), \text { for all } v \in \mathbb{Z}^{n}-\{0\}\right. \\
&\text { such that } \left.e_{1}, \cdots, e_{i-1}, v \text { can be extended to a basis of } \mathbb{Z}^{n}\right\} .
\end{aligned}
$$

For each $v \in \mathbb{Z}^{n}$, the condition $q_{i i} \leq Q(v)$ gives a linear equality on the coefficient matrix $\left(q_{i j}\right)$. Therefore, $\mathcal{D}_{n}^{M}$ is a convex subset of $\mathcal{P}_{n}$ or rather of the linear space $\mathcal{S}_{n}$ of symmetric $n \times n$-matrices. In particular, it is topologically

a cell. This is one place where the linear and convexity structures of $\mathcal{P}_{n}$ are crucial, and hence $\mathcal{P}_{n}$ instead of the subspace $\mathrm{SL}(n, \mathbb{R}) / \mathrm{SO}(n)$ is used. 
Proposition 4.26. The Minkowski reduction domain $\mathcal{D}_{n}^{M}$ is a fundamental domain for the action of $\mathrm{SL}(n, \mathbb{Z})$ on $\mathcal{P}_{n}=\mathrm{GL}^{+}(n, \mathbb{R}) / \mathrm{SO}(n)$ : for $\gamma \in$ $\mathrm{SL}(n, \mathbb{Z})$, the translates $\gamma \mathcal{D}_{n}^{M}$ cover the whole space $\mathcal{P}_{n}$, and without overlap in the interior.

To prove this, we need to show that for any $Q \in \mathcal{P}_{n}$, there exists $A \in$ $\mathrm{SL}(n, \mathbb{Z})$ such that the symmetric matrix

$$
Q[A]=A^{t} Q A
$$

is contained in $\mathcal{D}_{n}^{M}$. The idea for finding this matrix $A$ is to find its column vectors $v_{1}, \cdots, v_{n}$ one by one.

For this purpose, we introduced the notion of reduced basis of $\mathbb{Z}^{n}$. An ordered basis $v_{1}, \cdots, v_{n}$ of $\mathbb{Z}^{n}$ is called a reduced basis with respect to the positive quadratic form $Q$ if the following conditions are satisfied:

(1) The first vector $v_{1}$ is a nonzero vector $v$ in $\mathbb{Z}^{n}$ which minimizes the values $Q(v)$ :

$$
Q\left(v_{1}\right)=\min _{v \in \mathbb{Z}^{n}-\{0\}} Q(v) .
$$

Clearly such a vector $v_{1}$ has co-prime coordinates and can be extended to a basis of $\mathbb{Z}^{n}$.

(2) For each $i \geq 2, v_{i}$ is a vector among all vector $v$ such that $v_{1}, \cdots, v_{i-1}, v$ can be extended to a basis of $\mathbb{Z}^{n}$, and $Q\left(v_{i}\right)$ takes the minimum value:

$$
Q\left(v_{i}\right)=\min _{v \in \mathbb{Z}^{n}, v_{1}, \cdots, v_{i-1}, v \text { forms part of a basis of } \mathbb{Z}^{n}} Q(v) .
$$

It is clear that for any positive definite quadratic form $Q$, there exists an associated reduced basis of $\mathbb{Z}^{n}$. On the other hand, there may exist more than one reduced basis.

Given the above definition, a quadratic form $Q \in \mathcal{P}_{n}$ is Minkowski reduced, i.e., $Q \in \mathcal{D}_{n}^{M}$, if and only if the standard basis $e_{1}, \cdots, e_{n}$ of $\mathbb{Z}^{n}$ is a reduced basis.

For any $Q \in \mathcal{P}_{n}$, to construct a matrix $A \in \operatorname{SL}(n, \mathbb{Z})$ such that $Q[A] \in \mathcal{D}_{n}^{M}$, we take a reduced basis $v_{1}, \cdots, v_{n}$ of $\mathbb{Z}^{n}$ with respect to $Q$. Let $A$ be the matrix whose column vectors are $v_{1}, \cdots, v_{n}$. By reversing the sign of one vector if necessary, we can see that $A \in \mathrm{SL}(n, \mathbb{Z})$. Then the standard basis $e_{1}, \cdots, e_{n}$ forms a reduced basis of $Q[A]$. Therefore, $Q[A]$ is Minkowski reduced and contained in $\mathcal{D}_{n}^{M}$

In order to generalize this Minkowski reduction to the action of $\operatorname{Mod}_{g, n}$ on $\mathcal{T}_{g}$, we formulate it in terms of lattices $\Lambda \subset \mathbb{R}^{n}$, or equivalently tori $\mathbb{R}^{n} / \Lambda$. Given a marked lattice $\Lambda \subset \mathbb{R}^{n}$ with an ordered basis $v_{1}, \cdots, v_{n}$, let $A=$ $\left(v_{1}, \cdots, v_{n}\right)$ be the matrix formed from the basis. Then $A^{t} A$ is a positive definite quadratic form. Conversely, any positive definite quadratic form $Q$ 
can be written as $A^{t} A$, where $A$ is uniquely determined up to multiplication by elements of $\mathrm{O}(n)$. If we require $\operatorname{det} A=1$, then $A$ is uniquely determined up to multiplication by elements in $\mathrm{SO}(n)$. Let $\Lambda=A \mathbb{Z}^{n}, v_{1}=A e_{1}, \cdots, v_{n}=A e_{n}$. Then $\Lambda$ together with $v_{1}, \cdots, v_{n}$ is a marked lattice of $\mathbb{R}^{n}$. In terms of the torus (or flat manifold) $\mathbb{R}^{n} / \Lambda$, a marked lattice corresponds to a flat manifold $M=\mathbb{R}^{n} / \Lambda$ together with the choice of an ordered minimal set of generators of the fundamental group $\pi_{1}(M)$.

Given any lattice $\Lambda \subset \mathbb{R}^{n}$ endowed with the standard norm defined by the quadratic form $Q_{0}\left(x_{1}, \cdots, x_{n}\right)=\sum_{i=1}^{n} x_{i}^{2}$, an ordered basis $v_{1}, \cdots, v_{n}$ is called a reduced basis if the following conditions are satisfied:

(1) $v_{1}$ is of shortest length among all nonzero vectors of $\Lambda$. Clearly, such a vector $v_{1}$ is not a nontrivial integral multiple of any vector of $\Lambda$ and hence can be extended to a basis of $\Lambda$.

(2) For every $i \geq 2, v_{i}$ is of shortest norm among all vectors $v \in \Lambda$ such that $v_{1}, \cdots, v_{i-1}, v$ can be extended to a basis of $\Lambda$.

We note that in defining a reduced basis of $\mathbb{Z}^{n}$ with respect to a positive definite quadratic form $Q$, we use the standard lattice $\mathbb{Z}^{n}$ and a general positive quadratic form $Q$. On the other hand, for a reduced basis of a lattice $\Lambda$, we use the standard quadratic form $Q_{0}$. Of course, the two reduced bases correspond to each other under the identification between positive definite quadratic forms and marked lattices defined above.

Naturally, a marked lattice $\left(\Lambda ; v_{1}, \cdots, v_{n}\right)$ is called Minkowski reduced if the ordered basis $v_{1}, \cdots, v_{n}$ is a reduced basis of $\Lambda$.

Summarizing the above discussion, we have the following results:

Proposition 4.27. The Minkowski fundamental domain $\mathcal{D}_{n}^{M}$ for the action of $\mathrm{SL}(n, \mathbb{Z})$ on $\mathcal{P}_{n}=\mathrm{GL}^{+}(n, \mathbb{R}) / \mathrm{SO}(n)$ is characterized by the following equivalent conditions:

(1) A positive quadratic form $Q \in \mathcal{P}_{n}$ is contained in $\mathcal{D}_{n}^{M}$ if and only if the standard basis $e_{1}, \cdots, e_{n}$ is a Minkowski reduced basis of $\mathbb{Z}^{n}$ with respect to $Q$.

(2) A marked lattice $\left(\Lambda ; v_{1}, \cdots, v_{n}\right)$ is contained in $\mathcal{D}_{n}^{M}$ if and only if the basis $v_{1}, \cdots, v_{n}$ is Minkowski reduced.

Proof. (1) follows from the definition of $\mathcal{D}_{n}^{M}$, and (2) follows from the fact that if $\left(\Lambda ; v_{1}, \cdots, v_{n}\right)$ is a marked basis, then the standard basis $e_{1}, \cdots, e_{n}$ of $\mathbb{Z}^{n}$ is reduced with respect to the positive definite quadratic form $Q=A^{t} A$, where $A=\left(v_{1}, \cdots, v_{n}\right)$, if and only if $v_{1}, \cdots, v_{n}$ forms a reduced basis.

Proposition 4.26 only gives the most basic properties of the Minkowski fundamental domain. By definition, the Minkowski reduction domain is defined by infinitely many inequalities: for every $i, q_{i i} \geq Q(v)$, where $v$ is any vector 
$v \in \mathbb{Z}^{n}-\{0\}$ such that $e_{1}, \cdots, e_{i-1}, v$ can be extended to a basis of $\mathbb{Z}^{n}$. Of course, there are in general infinitely many such vectors $v$.

A natural question is whether finitely many inequalities are sufficient. The positive answer is an important result of Minkowski. We list this and other important results in the following proposition.

Proposition 4.28. The Minkowski reduction domain $\mathcal{D}_{n}^{M}$ is defined by finitely many inequalities and is hence a convex polyhedral cone with finitely many faces. The tiling of the space $\mathcal{P}_{n}$ of positive definite quadratic forms by the translates $\gamma \mathcal{D}_{n}^{M}, \gamma \in \mathrm{SL}(n, \mathbb{Z})$, is locally finite and each translate meets only finitely many others; hence $\mathcal{D}_{n}^{M}$ is a fundamental domain satisfying both the locally finite and globally finite conditions. The intersection of $\mathcal{D}_{n}^{M}$ with the subspace $S \mathcal{P}_{n}$ of $\mathcal{P}_{n}$ consisting of quadratic forms of determinant 1 is a cell, and the translates $\gamma\left(\mathcal{D}_{n}^{M} \cap S \mathcal{P}_{n}\right), \gamma \in \mathrm{SL}(n, \mathbb{Z})$, give an equivariant $C W$ complex structure of $\mathrm{SL}(n, \mathbb{R}) / \mathrm{SO}(n)=S \mathcal{P}_{n}$ with respect to $\mathrm{SL}(n, \mathbb{Z})$.

In order to prove this, the fundamental theorems of Minkowski in the geometry of numbers are needed. See [305], [392], [402] for details.

The (first) fundamental theorem of geometry of numbers is the following (see [392, p. 12] [158] [305]):

Proposition 4.29. If a bounded convex domain $K$ of $\mathbb{R}^{n}$ that contains the origin and is symmetric with respect to the origin has volume $\operatorname{vol}(K)>2^{n}$, then $K$ contains at least one zero point of $\mathbb{Z}^{n}$.

An immediate corollary is the following:

Proposition 4.30. Let $\operatorname{vol}\left(B_{1}\right)$ be the volume of the unit ball in $\mathbb{R}^{n}$ with respect to the standard metric. Then for any lattice $\Lambda$ of $\mathbb{R}^{n}$ of covolume 1 , there exists a nonzero vector $v \in \Lambda$ such that

$$
\|v\|^{n} \leq \frac{2^{n}}{\operatorname{vol}\left(B_{1}\right)} .
$$

In particular, for any reduced basis $v_{1}, \cdots, v_{n}$ of $\Lambda$,

$$
\left\|v_{1}\right\|^{n} \leq \frac{2^{n}}{\operatorname{vol}\left(B_{1}\right)} .
$$

The question of whether the norms of vectors in a reduced basis can be uniformly bounded is natural and it has also a positive answer [392, First Finiteness Theorem, p. 99]:

Proposition 4.31. For any lattice $\Lambda \subset \mathbb{R}^{n}$ of covolume 1 and any reduced basis $v_{1}, \cdots, v_{n}$, the norms of the basis vectors satisfy the bounds:

$$
\frac{2^{n}}{\operatorname{vol}\left(B_{1}\right)} \frac{1}{n !} \leq\left\|v_{1}\right\| \cdots\left\|v_{n}\right\| \leq \frac{2^{n}}{\operatorname{vol}\left(B_{1}\right)}\left(\frac{3}{2}\right)^{n(n-1) / 2} .
$$


In some sense, this result says that a reduced basis tends to be as orthogonal as possible. In Propositions 4.30 and 4.31, we could have stated similar results for general lattices of $\mathbb{R}^{n}$ instead of covolume 1 . The point of these results is that the bounds on norms of the vectors in a reduced basis are independent of lattices but only depend on the co-volumes of the lattices.

These results seemed to motivate the existence of pants decompositions of hyperbolic surfaces such that lengths of geodesics in the pants decompositions are bounded by the Bers constants in Proposition 5.25 below.

Remark 4.32. The second finiteness theorem of Minkowski in [392, p. 127] refers to the fact that the Minkowski reduction domain is defined by finitely many inequalities, which was mentioned in Proposition 4.28. Determining these inequalities explicitly is very difficult and has been only carried out for small values of $n$. See [369] [370] for summaries and references.

\subsection{Reduction theory for general arithmetic groups}

In the Minkowski reduction theory, an important role was played by the identification of the space $\mathrm{SL}(n, \mathbb{R}) / \mathrm{SO}(n)$ with the space of positive definite quadratic forms of determinant 1 , and also with the space of marked lattices of $\mathbb{R}^{n}$ of covolume 1 . Such a moduli interpretation of points of the symmetric space $\mathrm{SL}(n, \mathbb{R}) / \mathrm{SO}(n)$ and the locally symmetric space $\mathrm{SL}(n, \mathbb{Z}) \backslash \mathrm{SL}(n, \mathbb{R}) / \mathrm{SO}(n)$ is important in describing points in desired fundamental domains.

For a general symmetric space $X=G / K$ and an arithmetic group $\Gamma$ acting on it, there is no such moduli interpretation and hence there is no such notion of reduced points. As pointed out before, the Dirichlet fundamental domains are not suitable for various questions about $\Gamma \backslash X$.

We recall some general statements on the reduction theory for arithmetic groups as developed by Siegel, Borel \& Harish-Chandra, and Borel [57].

The key notion is that of Langlands decomposition of $\mathbb{Q}$-parabolic subgroups and the induced horospherical decomposition of symmetric spaces.

Fix a basepoint $x_{0}=K \in X=G / K$. For every $\mathbb{Q}$-parabolic subgroup $\mathbf{P}$ of $\mathbf{G}$, its real locus $P=\mathbf{P}(\mathbb{R})$ admits a $\mathbb{Q}$-Langlands decomposition with respect to $x_{0}$,

$$
P=N_{\mathbf{P}} A_{\mathbf{P}} M_{\mathbf{P}} \cong N_{\mathbf{P}} \times A_{\mathbf{P}} \times M_{\mathbf{P}}
$$

where $N_{\mathbf{P}}$ is the unipotent radical of $P, A_{\mathbf{P}}$ is the $\mathbb{Q}$-split component of $P$, $M_{\mathbf{P}}$ is a reductive group, and $A_{\mathbf{P}} M_{\mathbf{P}}$ is the Levi factor of $P$ invariant under the Cartan involution associated with $K$. Though $N_{\mathbf{P}}$ is canonically defined, $A_{\mathbf{P}}$ and $M_{\mathbf{P}}$ depend on the choice of the base point $x_{0}$.

Define the boundary symmetric space $X_{\mathbf{P}}$ associated with $P$ by

$$
X_{\mathbf{P}}=M_{\mathbf{P}} /\left(M_{\mathbf{P}} \cap K\right) .
$$


Then the Langlands decomposition of $P$ induces the horospherical decomposition of $X$ with respect to $P$ :

$$
X \cong N_{\mathbf{P}} \times A_{\mathbf{P}} \times X_{\mathbf{P}} .
$$

When $X=\mathrm{SL}(2, \mathbb{R}) / \mathrm{SO}(2) \cong \mathbf{H}^{2}$ and $P$ is the subgroup of upper triangular matrices, the horospherical decomposition corresponds to the $x, y$ coordinates of the upper half plane $\mathbf{H}^{2}$.

Let $\mathfrak{n}_{P}$ be the Lie algebra of $N_{\mathbf{P}}$, and $\mathfrak{a}_{\mathbf{P}}$ the Lie algebra of $A_{\mathbf{P}}$. The set of roots of the action of $\mathfrak{a}_{\mathbf{P}}$ on $\mathfrak{n}_{P}$ is denoted by $\Phi\left(A_{\mathbf{P}}, P\right)$. Then the subset

$$
\mathfrak{a}_{\mathbf{P}}^{+}=\left\{H \in \mathfrak{a}_{P} \mid \alpha(H)>0, \alpha \in \Phi\left(A_{\mathbf{P}}, P\right)\right\}
$$

is called the positive chamber of $\mathfrak{a}_{\mathbf{P}}$ determined by $P$. Similarly,

$$
A_{\mathbf{P}}^{+}=\exp \mathfrak{a}_{\mathbf{P}}^{+}
$$

is called the positive chamber of $A_{\mathbf{P}}$. For any $t>0$, define

$$
A_{\mathbf{P}, t}=\left\{a \in A_{\mathbf{P}} \mid e^{\alpha(\log a)}>t\right\} .
$$

This is a shift of the positive chamber $A_{\mathbf{P}}^{+}$.

Definition 4.33. For any bounded subsets $U \subset N_{\mathbf{P}}$ and $V \subset X_{\mathbf{P}}$, the subset of $X$ corresponding to $U \times A_{\mathbf{P}, t} \times V$ under the horopsherical decomposition in Equation (2) is called a Siegel set associated with $\mathbf{P}$ and denoted by $\mathcal{S}_{\mathbf{P}, t}$.

The basic result in the reduction theory of arithmetic groups is the following. See [57] for a proof and more details.

Proposition 4.34. Let $\mathbf{G}$ be a linear semisimple algebraic group defined over $\mathbb{Q}$, and $\Gamma$ an arithmetic subgroup of $\mathbf{G}(\mathbb{Q})$. Then there are only finitely many $\Gamma$ conjugacy classes of $\mathbb{Q}$-parabolic subgroups of $\mathbf{G}$. Let $\mathbf{P}_{1}, \cdots, \mathbf{P}_{k}$ be representatives of these conjugacy classes. Then there are Siegel sets $\mathcal{S}_{\mathbf{P}_{1}, t_{1}}, \cdots, \mathcal{S}_{\mathbf{P}_{k}, t_{k}}$ such that their union $\mathcal{S}=\mathcal{S}_{\mathbf{P}_{1}, t_{1}} \cup \cdots \cup \mathcal{S}_{\mathbf{P}_{k}, t_{k}}$ is a fundamental set for $\Gamma$ in the following sense:

(1) $\cup_{\gamma \in \Gamma} \Gamma \mathcal{S}=X$.

(2) For any $g \in \mathbf{G}(\mathbb{Q})$, the set $\{\gamma \in \Gamma \mid \gamma \mathcal{S} \cap g \mathcal{S} \neq \emptyset\}$ is finite.

The finiteness condition in (2) is called the Siegel finiteness condition and is a key result in the reduction theory for arithmetic groups.

This basic result has many consequences and applications, which will be explained in later sections. We point out some immediate ones in this subsection. The first is the following.

Corollary 4.35. Under the above assumption, the locally symmetric space $\Gamma \backslash X$ has finite volume. 
The basic reason is that a Siegel set $\mathcal{S}_{\mathbf{P}, t}$ is a product $U \times A_{\mathbf{P}, t} \times V$, and the invariant metric of $X$ has a simple expression in horospherical coordinates. (See [59] or [60] for example.) Hence it it can easily be shown that $\mathcal{S}_{\mathbf{P}, t}$ has finite volume.

For example, when $\Gamma=\mathrm{SL}(2, \mathbb{Z})$ and $\mathbf{P}$ is the subgroup of upper triangular matrices in $\operatorname{SL}(2, \mathbb{R})$, then $\mathbf{P}$ is a $\mathbb{Q}$-parabolic subgroup of $\mathrm{SL}(2, \mathbb{C})$, and a Siegel set associated with $\mathbf{P}$ is a vertical strip $\left\{x+i y \mid a_{1} \leq x \leq a_{2}, y>b\right\}$ for some $a_{1}, a_{2} \in \mathbb{R}, b>0$. Clearly such a region has finite hyperbolic area.

Since any Siegel set corresponding to the improper $\mathbb{Q}$-parabolic subgroup $\mathbf{G}$ is bounded and the existence of proper $\mathbb{Q}$-parabolic subgroups of $\mathbf{G}$ is equivalent to the positivity of the $\mathbb{Q}$-rank of $\mathbf{G}$, we obtain the following consequence, which was a conjecture of Godement and proved independently by Borel \& Harish-Chandra, and Mostow \& Tamagama.

Corollary 4.36. The locally symmetric space $\Gamma \backslash X$ is compact if and only if the $\mathbb{Q}$-rank of $\Gamma$ is equal to 0 , which is also equivalent to the fact that $\Gamma$ does not contain any nontrivial unipotent element.

Recall that the $\mathbb{Q}$-Tits building $\Delta_{\mathbb{Q}}(\mathbf{G})$ of $\mathbf{G}$ is an infinite simplicial complex whose simplices are parametrized by proper $\mathbb{Q}$-parabolic subgroups of $\mathbf{G}$ satisfying the following conditions:

(1) For every $\mathbb{Q}$-parabolic subgroup $\mathbf{P}$, denote its corresponding simplex by $\sigma_{\mathbf{P}}$. Then $\sigma_{\mathbf{P}}$ is of dimension 0 if and only if $\mathbf{P}$ is a maximal $\mathbb{Q}$-parabolic subgroup of $\mathbf{G}$.

(2) For every pair of $\mathbb{Q}$-parabolic subgroups $\mathbf{P}_{1}, \mathbf{P}_{2}, \sigma_{\mathbf{P}_{1}}$ is a face of $\sigma_{\mathbf{P}_{2}}$ if and only if $\mathbf{P}_{1}$ contains $\mathbf{P}_{2}$. In particular, the vertices of any simplex $\sigma_{\mathbf{P}}$ correspond to maximal $\mathbb{Q}$-parabolic subgroups that contain $\mathbf{P}$, and the intersection of these maximal $\mathbb{Q}$-parabolic subgroups is equal to $\mathbf{P}$.

Since $\mathbf{G}(\mathbb{Q})$ and hence $\Gamma$ act on the set of $\mathbb{Q}$-parabolic subgroups by conjugation, they also act on the Tits building $\Delta_{\mathbb{Q}}(\mathbf{G})$ by simplicial homeomorphisms. Another corollary of the reduction theory is the following.

Corollary 4.37. The quotient $\Gamma \backslash \Delta_{\mathbb{Q}}(\mathbf{G})$ is a finite simplicial complex.

The theory of linear algebraic groups implies that $\Delta_{\mathbb{Q}}(\mathbf{G})$ satisfies the axioms for Tits buildings. In particular, any two simplices are contained in an apartment, which is a finite simplicial complex and whose underlying space is the unit sphere in $\mathfrak{a}_{\mathbf{P}}$ for a minimal $\mathbb{Q}$-parabolic subgroup $\mathbf{P}$ of $\mathbf{G}$. It follows that the Tits building $\Delta_{\mathbb{Q}}(\mathbf{G})$ is connected if and only if the $\mathbb{Q}$-rank is at least 2. Combining this with the reduction theory in Proposition 4.34 (or using the Borel-Serre compactification of $\Gamma \backslash X$ defined later), we can prove the following result [204]. 
Proposition 4.38. The locally symmetric space $\Gamma \backslash X$ is connected at infinity, i.e., has one end, if and only if the $\mathbb{Q}$-rank of $\Gamma \backslash X$ is at least 2. When the $\mathbb{Q}$-rank is equal to 1 , the ends of $\Gamma \backslash X$ are in one-to-one correspondence with the $\Gamma$-conjugacy classes of $\mathbb{Q}$-parabolic subgroups.

\subsection{Precise reduction theory for arithmetic groups}

Though the reduction theory in Proposition 4.34 suffices for many applications, it is an interesting and important problem to get fundamental domains, instead of rough fundamental domains (or fundamental sets in the sense of [57]), that can be conveniently described, for example, in terms of horopsherical decompositions with respect to $\mathbb{Q}$-parabolic subgroups or as special subsets of Siegel sets.

Another natural question is whether there is a generalization of Minkowski reduction for general arithmetic subgroups.

In this subsection, we summarize cases of arithmetic groups for which more precise descriptions of and results on fundamental sets or fundamental domains are available and discuss approaches to obtain them.

Linear symmetric spaces

For the symmetric space $X=\mathrm{GL}^{+}(n, \mathbb{R}) / \mathrm{SO}(n)$ and the arithmetic group $\operatorname{SL}(n, \mathbb{Z})$, there is another reduction theory developed by Voronoi using perfect quadratic forms. (See [298] for a summary and references. See also [14] and [15, Chapter 1]).

The symmetric space $\mathrm{GL}^{+}(n, \mathbb{R}) / \mathrm{SO}(n)$ is special in that it is the selfadjoint homogeneous cone of positive definite quadratic forms in the vector space of all symmetric bilinear forms, and the symmetric space $\operatorname{SL}(n, \mathbb{R}) / \mathrm{SO}(n)$ is a homothety section of the cone. The collection of perfect quadratic forms induces an $\mathrm{SL}(n, \mathbb{Z})$-equivariant polyhedral cone decomposition of the cone $\mathrm{GL}^{+}(n, \mathbb{R}) / \mathrm{SO}(n)$, which is different from the equivariant decomposition arising from the translates of the Minkowski reduction domains.

In general, a symmetric space $X=G / K$ is called a linear symmetric space if it is a self-adjoint homogeneous cone or a homethety section of such a cone. For such a linear symmetric space $X$ and an arithmetic subgroup $\Gamma \subset G, X$ admits a $\Gamma$-equivariant decomposition into simplicial cones such that there are only finitely many orbits of simplices. This implies that $\Gamma$ admits a fundamental domain that is a finite union of simplicial cones. Such simplicial decompositions are essential for toroidal compactifications of Hermitian locally symmetric spaces. See [15, Chapter 1] and [14].

There are also generalizations of Minkowski reduction theory to other groups, for example, $\mathrm{SL}\left(n, \mathcal{O}_{k}\right)$, where $k$ is a number field and $\mathcal{O}_{k}$ is the ring of integers of $k$. See [418] [419] [183] [247, Chap I, §4]. 
For $\mathrm{GL}^{+}(n, \mathbb{R}) / \mathrm{SO}(n)$, there is also the Venkov reduction. The idea is similar to the Dirichlet fundamental domain. Pick a positive definite matrix $Q \in \mathrm{GL}^{+}(n, \mathbb{R}) / \mathrm{SO}(n)$. Then the inner product with this matrix $Q$ defines a positive function on $\mathrm{GL}^{+}(n, \mathbb{R}) / \mathrm{SO}(n)$ :

$$
d_{Q}(A)=\operatorname{Tr}(A Q), \quad A \in \mathrm{GL}^{+}(n, \mathbb{R}) / \mathrm{SO}(n) .
$$

For every $\mathrm{SL}(n, \mathbb{Z})$-orbit in $\mathrm{GL}^{+}(n, \mathbb{R}) / \mathrm{SO}(n)$, consider the points where $d_{Q}$ takes minimum values. Then the union of such points gives the Venkov reduction domain. It is a convex polyhedron bounded by finitely many faces. See [369] [370].

A generalization of this reduction theory to general linear symmetric space is given in [243].

Symplectic groups

The Siegel upper half space

$\mathfrak{h}_{g}=\{X+i Y \mid X, Y$ real $g \times g$ symmetric matrices, $Y>0\} \cong \mathrm{Sp}(2 g, \mathbb{R}) / \mathrm{U}(g)$

is not a linear symmetric space. But for $\Gamma=\operatorname{Sp}(2 g, \mathbb{Z})$, a fundamental domain was explicitly determined by Siegel by making use of the reduction theory for $\mathrm{SL}(n, \mathbb{Z})$. The proof is similar in some sense to that used in identifying the classical fundamental domain for $\mathrm{SL}(2, \mathbb{Z})$. See [273] for details.

For any finite index subgroup $\Gamma$ of $\operatorname{Sp}(2 g, \mathbb{Z})$, a finite union of suitable translates of the fundamental domain for $\operatorname{Sp}(2 g, \mathbb{Z})$ gives a fundamental domain for $\Gamma$. For any other arithmetic subgroup $\Gamma$ of $\operatorname{Sp}(2 g, \mathbb{Q})$, a conjugate of $\Gamma$ by an element of $\operatorname{Sp}(2 g, \mathbb{Q})$ is contained in $\operatorname{Sp}(2 g, \mathbb{Z})$, and we can also obtain an explicit fundamental domain for $\Gamma$ by taking a finite union of suitable translates of the fundamental domain for $\operatorname{Sp}(2 g, \mathbb{Z})$.

As in the case of arithmetic subgroups of $\operatorname{SL}(2, \mathbb{Q})$, it is desirable to obtain a connected fundamental domain instead of a finite union of domains.

Fundamental domains in complex hyperbolic spaces

As mentioned before, Dirichlet fundamental domains for Fuchsian groups acting on the Poincaré half plane have good properties and are useful in studying algebraic structures of Fuchsian groups. For other rank one symmetric spaces, Dirichlet fundamental domains are more complicated. For example, see [148] for Dirichlet fundamental domains in complex hyperbolic spaces. Many results on explicit fundamental domains have been obtained, see the papers [121], [342], [138], [139] [341].

Equivariant tilings of symmetric spaces

The Minkowski and Voronoi reduction theories induce equivariant cell decompositions of the symmetric spaces $\mathrm{GL}^{+}(n, \mathbb{R}) / \mathrm{SO}(n)$ and $\mathrm{SL}(n, \mathbb{R}) / \mathrm{SO}(n)$. 
For general symmetric spaces $X=G / K$ and arithmetic groups acting on them, coarser equivariant tilings (or decompositions) are known (see [371] for precise statements of the results and references). Such an equivariant decomposition is important for the Arthur-Selberg trace formula but does not give rise to a well-defined fundamental domain. The reason is that each tile admits an infinite stabilizer in $\Gamma$, and finding a fundamental domain of such a stabilizer in each tile is not obvious but it might be less difficult than finding a fundamental domain for $\Gamma$ since it might be reduced to lower dimensional cases.

On the other hand, by picking a fundamental domain for each tile, we can get a fundamental domain for $\Gamma$ which is a union of pieces parametrized by representatives of $\Gamma$-conjugacy classes of $\mathbb{Q}$-parabolic subgroups. The piece corresponding to the improper $\mathbb{Q}$-parabolic subgroup $\mathbf{G}$ is bounded, and each piece for a proper $\mathbb{Q}$-parabolic subgroup $\mathbf{P}$ is contained in a Siegel set of $\mathbf{P}$. Though not canonically defined, such a fundamental domain for $\Gamma$ is useful for many applications. This reduction theory is usually called precise reduction theory. (See [61] for example.)

In the classical reduction theory described in Proposition 4.34, Siegel sets of non-minimal $\mathbb{Q}$-parabolic subgroups are not really needed. In fact, the union of suitable Siegel sets associated with representatives of $\Gamma$-conjugacy classes of minimal $\mathbb{Q}$-parabolic subgroups of $\mathbf{G}$ gives a fundamental set of $\Gamma$. On the other hand, in constructing a fundamental domain from the above equivariant tiling, we do need all $\mathbb{Q}$-parabolic subgroups of $\mathbf{G}$, including the improper parabolic subgroup $\mathbf{G}$.

When the $\mathbb{Q}$-rank of $\mathbf{G}$ is equal to 1 , the fundamental domain constructed in this way is related to the one constructed in the next paragraph, though the point of view is slightly different and the latter is more intrinsic in some sense.

\section{Intrinsic fundamental domains for $\mathbb{Q}$-rank 1 arithmetic subgroups}

If the $\mathbb{Q}$-rank of $\mathbf{G}$ is equal to 1 , then we can get a fundamental domain of $\Gamma$ by using the height functions on $X$ associated with $\mathbb{Q}$-parabolic subgroups.

To explain the idea, we interpret the Dirichlet fundamental domain $D\left(x_{0}, \Gamma\right)$ in a slightly different way. By assumption, the base-point $x_{0}$ is not fixed by any nontrivial element of $\Gamma$. For each point $\gamma x_{0}$ in the orbit $\Gamma x_{0}$, define a function on $X$ :

$$
d_{\gamma x_{0}}(x)=d\left(x, \gamma x_{0}\right) .
$$

Using this function, for each point $\gamma x_{0}$, we define a region

$$
\Omega\left(\gamma x_{0}\right)=\left\{x \in X \mid d_{\gamma x_{0}}(x) \leq d_{y}(x), \text { for all } y \in \Gamma x_{0}\right\} .
$$

Clearly, $\Omega\left(\gamma x_{0}\right)=\gamma \Omega\left(x_{0}\right)$. Then these subsets give a $\Gamma$-equivariant decomposition of $X$, and $\Omega\left(x_{0}\right)$ is the Dirichlet domain $\bar{D}\left(x_{0}, \Gamma\right)$. Since no nontrivial 
element of $\Gamma$ fixes $x_{0}$ or any point of the orbit $\Gamma x_{0}, \Gamma$ acts simply transitively on these subsets.

Suppose that there is a family of functions $f_{i}, i \in I$, on $X$ that is stable under $\Gamma$, i.e., for any $\gamma \in \Gamma$ and $f_{i}, f_{i}(\gamma \cdot x)$ is equal to $f_{j}(x)$ for some $j \in I$. There are two cases.

Case 1. Assume that for every $x \in X, \inf _{i \in I} f_{i}(x)$ is realized. For each function $f_{i}$, define a region

$$
\tilde{\Omega}_{i}=\left\{x \in X \mid f_{i}(x) \leq f_{j}(x), j \in I\right\} .
$$

Case 2. Assume that for every $x \in X$, $\sup _{i \in I} f_{i}(x)$ is realized, then for each function $f_{i}$, define a region

$$
\tilde{\Omega}_{i}=\left\{x \in X \mid f_{i}(x) \geq f_{j}(x), j \in I\right\} .
$$

These subsets $\tilde{\Omega}_{i}, i \in I$, form a family which is stable under $\Gamma$. On the other hand, unlike the previous case, each of these subsets is not yet a fundamental domain of $\Gamma$.

To find a fundamental domain for $\Gamma$, denote the stabilizer of $f_{i}$ in $\Gamma$ by $\Gamma_{i}$; then $\Gamma_{i}$ acts on $\tilde{\Omega}_{i}$. Let $\Omega_{i}$ be a fundamental domain of $\Gamma_{i}$ in $\tilde{\Omega}_{i}$.

Assume that there are only finitely many $\Gamma$-orbits in $\left\{f_{i} \mid i \in I\right\}$. Let $f_{i_{1}}, \cdots, f_{i_{k}}$ be a set of representatives. Then it can be shown that the union $\Omega_{i_{1}} \cup \cdots \cup \Omega_{i_{k}}$ is a fundamental domain for the $\Gamma$-action on $X$.

If we take $f_{i}$ to be the distance function $d_{\gamma x_{0}}$ above such that $x_{0}$ is not fixed by any nontrivial element of $\Gamma$, then the stabilizer of each function $f_{i}$ is trivial, and this construction specializes to the previous case of Dirichlet fundamental domains. Note that in this case, there is only one $\Gamma$-orbit in the collection of functions $d_{\gamma x_{0}}$.

If the $\mathbb{Q}$-rank of $\mathbf{G}$ is equal to 1 , take $\mathcal{P}$ to be the set of proper $\mathbb{Q}$-parabolic subgroups $\mathbf{P}$ of $\mathbf{G}$. For each $\mathbb{Q}$-parabolic subgroup $\mathbf{P}$, let $\alpha \in \Phi\left(A_{\mathbf{P}}, P\right)$ be the unique short root. Choose a $\mathbb{Q}$-Langlands decomposition $P=N_{\mathbf{P}} A_{\mathbf{P}} M_{\mathbf{P}}$, and hence an associated horopherical decomposition of $X$ :

$$
X \cong N_{\mathbf{P}} \times A_{\mathbf{P}} \times X_{\mathbf{P}}, \quad x \mapsto\left(n_{\mathbf{P}}(x), a_{\mathbf{P}}(x), z_{\mathbf{P}}(x)\right) .
$$

Define a height function on $X$ associated with the parabolic subgroup $\mathbf{P}$ by

$$
h_{\mathbf{P}}(x)=\alpha\left(\log a_{\mathbf{P}}\right) .
$$

Note that the Langlands decomposition and horospherical decomposition of $P$ depend on the choice of a basepoint in $X$ (See Equation 2). A different choice of the basepoint will lead to a shift of the height function $h_{\mathbf{P}}$. It turns out that there are choices such that the family of height functions $h_{\mathbf{P}}$ is stable under $\Gamma$. The basic idea is as follows. By the reduction theory (Proposition 4.34), there are only finitely many $\Gamma$-conjugacy classes of $\mathbb{Q}$-parabolic subgroups of G. Fix some representatives of these conjugacy classes and choose arbitrary basepoints for them. Then there are choices of basepoints, or equivalently 
height functions, for all other parabolic subgroups such that the family of height functions $h_{\mathbf{P}}$ is stable under $\Gamma$ (see [428]).

For each height function $h_{\mathbf{P}}(x)$, its stabilizer is equal to $\Gamma \cap P$ which is a uniform lattice in $N_{\mathbf{P}} M_{\mathbf{P}}$. (Recall that $P=N_{\mathbf{P}} A_{\mathbf{P}} M_{\mathbf{P}}$ is the $\mathbb{Q}$-Langlands decomposition of $P$.) Since fundamental domains for the $\Gamma \cap P$-action on $N_{\mathbf{P}} \times X_{\mathbf{P}}$ and $X$ can be described relatively easily in terms of the horospherical decomposition of $X$ with respect to $\mathbf{P}$, the above approach can be used to obtain fundamental domains for $\Gamma$ when the $\mathbb{Q}$-rank of $\mathbf{G}$ is equal to 1.

For each $\mathbf{P} \in \mathcal{P}$ and for every $x \in X$, by the reduction theory, $\sup _{\mathbf{P} \in \mathcal{P}} h_{\mathbf{P}}(x)$ is realized. Then we define a domain $\tilde{\Omega}_{\mathbf{P}}$ for each $\mathbf{P}$ as in the $\mathbb{Q}$-rank 1 case above by

$$
\tilde{\Omega}_{\mathbf{P}}=\left\{x \in X \mid h_{\mathbf{P}}(x) \geq h_{\mathbf{P}^{\prime}}(x), \mathbf{P}^{\prime} \in \mathbf{P}\right\} .
$$

In the horospherical decomposition $X=N_{\mathbf{P}} \times A_{\mathbf{P}} \times X_{\mathbf{P}} \cong N_{\mathbf{P}} \times X_{\mathbf{P}} \times A_{\mathbf{P}}$, the stabilizer of $h_{\mathbf{P}}$ in $\Gamma$ is equal to $\Gamma \cap P=\Gamma \cap N_{\mathbf{P}} M_{\mathbf{P}}$. It acts cocompactly on $N_{\mathbf{P}} \times X_{\mathbf{P}}$ and leaves the component $A_{\mathbf{P}}$ fixed. Let $\Omega_{\Gamma \cap N_{\mathbf{P}} M_{\mathbf{P}}}$ be a compact fundamental domain in $N_{\mathbf{P}} \times X_{\mathbf{P}}$ for the stabilizer. Define

$$
\Omega_{\mathbf{P}}=\tilde{\Omega}_{\mathbf{P}} \cap\left(\Omega_{\Gamma \cap N_{\mathbf{P}} M_{\mathbf{P}}} \times A_{\mathbf{P}}\right) .
$$

Let $\mathbf{P}_{1}, \cdots, \mathbf{P}_{m}$ be a set of representatives of $\Gamma$-conjugacy classes of $\mathbb{Q}$-parabolic subgroups. Then the union $\Omega_{\mathbf{P}_{1}} \cup \cdots \cup \Omega_{\mathbf{P}_{m}}$ is a fundamental domain for the $\Gamma$-action on $X$. It can be shown that each of the domains $\Omega_{\mathbf{P}_{1}}, \cdots, \Omega_{\mathbf{P}_{m}}$ is contractible. Hence the topology of the fundamental domain is relatively simple.

The Hilbert modular groups are some of the most important examples of $\mathbb{Q}$-rank 1 arithmetic groups. There has been a lot of work on their fundamental domains. See [393, Chap. III, §2] [97] [98] [408, p. 8-11].

\section{Intrinsic fundamental domain for higher $\mathbb{Q}$-rank arithmetic subgroups}

A natural problem is to obtain a generalization of the Minkowski reduction theory to a general arithmetic subgroup $\Gamma$ by picking out points of $X$ that are minimal (or rather maximal) with respect to a family of height functions.

Given the result discussed above for the $\mathbb{Q}$-rank 1 case, it is natural to define height functions $h_{\mathbf{P}}$ for all maximal $\mathbb{Q}$-parabolic subgroups $\mathbf{P}$ of $\mathbf{G}$ and use them to define a reduced domain analogous to the Minkowski reduction domain. Let $\mathcal{P}_{\max }$ be the set of all maximal $\mathbb{Q}$-parabolic subgroups. For every $\mathbf{P} \in \mathcal{P}_{\max }$, we can also define a domain $\tilde{\Omega}_{\mathbf{P}}$ as above. But the stabilizer of $h_{\mathbf{P}}$ in $\Gamma$ is $\Gamma \cap P=\Gamma \cap N_{\mathbf{P}} M_{\mathbf{P}}$, which does not act cocompactly on $N_{\mathbf{P}} \times X_{\mathbf{P}}$ and involves a non-compact locally symmetric space $\Gamma_{M_{\mathbf{P}}} \backslash X_{\mathbf{P}}$. On the other hand, if we do this by induction and find $\Gamma_{M_{\mathbf{P}}}$-fundamental domains in $X_{\mathbf{P}}$ and hence fundamental domains of $\Gamma \cap N_{\mathbf{P}} M_{\mathbf{P}}$, then we can follow the steps for the $\mathbb{Q}$-rank 1 case and define $\Omega_{\Gamma \cap N_{\mathbf{P}} M_{\mathbf{P}}}$ and $\Omega_{\mathbf{P}}$ etc. 
Here is another approach that avoids inductive difficulties. In some sense, it is a generalization of the Minkowski reduction theory. The idea is as follows. For every $x \in X$, define an ordered set of maximal parabolic subgroups $\mathbf{P}_{1}, \cdots, \mathbf{P}_{r}$ such that $\mathbf{P}_{1} \cap \cdots \cap \mathbf{P}_{r}$ is a minimal $\mathbb{Q}$-parabolic subgroup of $\mathbf{G}$, where $r$ is the $\mathbb{Q}$-rank of $\mathbf{G}$. The important point is that the stabilizer in $\Gamma$ of the ordered set of height functions $h_{\mathbf{P}_{1}}, \cdots, h_{\mathbf{P}_{r}}$ is equal to $\Gamma \cap P$, where $\mathbf{P}=\mathbf{P}_{1} \cap \cdots \cap \mathbf{P}_{r}$. Since $\mathbf{P}$ is a minimal $\mathbb{Q}$-parabolic subgroup, $\Gamma \cap P=\Gamma \cap N_{\mathbf{P}} M_{\mathbf{P}}$ acts cocompactly on $N_{\mathbf{P}} \times X_{\mathbf{P}}$ and it is easier to find its fundamental domains in $N_{\mathbf{P}} \times X_{\mathbf{P}}$.

For every point $x$, choose $\mathbf{P}_{1} \in \mathcal{P}_{\max }$ such that

$$
h_{\mathbf{P}_{1}}(x) \geq h_{\mathbf{P}}(x)
$$

for all $\mathbf{P} \in \mathcal{P}_{\max }$. Consider all maximal parabolic subgroups $\mathbf{P}$ such that $\mathbf{P}_{1} \cap \mathbf{P}$ is a $\mathbb{Q}$-parabolic subgroup of $\mathbf{G}$ and pick one, denoted by $\mathbf{P}_{2}$, such that $h_{\mathbf{P}_{2}}(x)$ has a maximum value. Suppose that $\mathbf{P}_{1}, \cdots, \mathbf{P}_{i}$ have been picked and $\mathbf{P}_{1} \cap \cdots \cap \mathbf{P}_{i}$ is a non-minimal parabolic subgroup. Consider all maximal $\mathbb{Q}$-parabolic subgroups $\mathbf{P}$ such that $\mathbf{P}_{1} \cap \cdots \cap \mathbf{P}_{i} \cap \mathbf{P}$ is a $\mathbb{Q}$-parabolic subgroup of $\mathbf{G}$ and pick one $\mathbf{P}_{i+1}$ such that $h_{\mathbf{P}_{i+1}}(x)$ takes a maximum value.

The ordered sequence $\mathbf{P}_{1}, \cdots, \mathbf{P}_{r}$ is called a reduced sequence of maximal $\mathbb{Q}$-parabolic subgroups for the point $x$. In the above procedure, the choices of $\mathbf{P}_{1}, \cdots, \mathbf{P}_{r}$ are not unique for points $x$ when several height functions take the maximum value. On the other hand, for a generic point $x$, there is a unique maximum height function, and the ordered groups $\mathbf{P}_{1}, \cdots, \mathbf{P}_{r}$ are unique for $x$.

For every ordered sequence of $\mathbf{P}_{1}, \cdots, \mathbf{P}_{r}$ as above such that $\mathbf{P}_{1} \cap \cdots \cap \mathbf{P}_{r}$ is a minimal $\mathbb{Q}$-parabolic subgroup, we define a region

$$
\tilde{\Omega}_{\mathbf{P}_{1}, \cdots, \mathbf{P}_{r}}=\left\{x \in X \mid \mathbf{P}_{1}, \cdots, \mathbf{P}_{r} \text { is the reduced sequence for } x\right\} .
$$

Let $\mathbf{P}=\mathbf{P}_{1} \cap \cdots \cap \mathbf{P}_{r}$. Let $\Omega_{\Gamma, \mathbf{P}}$ be a fundamental domain for $\Gamma \cap N_{\mathbf{P}} M_{\mathbf{P}}$ acting on $N_{\mathbf{P}} \times X_{\mathbf{P}}$. Define

$$
\Omega_{\mathbf{P}_{1}, \cdots, \mathbf{P}_{r}}=\tilde{\Omega}_{\mathbf{P}_{1}, \cdots, \mathbf{P}_{r}} \cap \Omega_{\Gamma, \mathbf{P}}
$$

By the reduction theory, there are only finitely many conjugacy classes of such ordered $r$-tuples $\mathbf{P}_{1}, \cdots, \mathbf{P}_{r}$. Pick and fix representatives of these classes. Then the union of their domains $\Omega_{\mathbf{P}_{1}, \cdots, \mathbf{P}_{r}}$ is a fundamental domain for the $\Gamma$-action on $X$. It can also be shown that each domain $\Omega_{\mathbf{P}_{1}, \cdots, \mathbf{P}_{r}}$ is contractible, by deforming along the orbits of the geodesic action of $A_{\mathbf{P}}$ on $X$, where $\mathbf{P}=\mathbf{P}_{1} \cap \cdots \cap \mathbf{P}_{r}$ as above. 


\subsection{Combinatorial properties of arithmetic groups: finite presentation and bounded generation}

An immediate application of the reduction theory (Proposition 4.34), in particular, the Siegel finiteness, is the finite generation of arithmetic groups.

This follows from the following general result.

Lemma 4.39. If $X$ is a connected topological space and a $\Gamma$-action on $X$ admits a rough open (or closed) fundamental domain $\Omega$, then the set $S=\{\gamma \in$ $\gamma \Omega \cap \Omega \neq \emptyset\}$ generates $\Gamma$. If this set $S$ is finite, i.e., if $\Omega$ satisfies the global finiteness condition, then $\Gamma$ is finitely generated.

The idea of the proof is that if the subgroup $\Gamma^{\prime}$ generated by these elements in $S$ is not equal to $\Gamma$, then the two unions of translates of $\Omega, \cup_{\gamma \in \Gamma^{\prime}} \gamma \Omega$ and $\cup_{\gamma \in \Gamma-\Gamma^{\prime}} \gamma \Omega$, give a disjoint decomposition of $X$ into two open subsets. This contradicts the assumption that $X$ is connected. See [390], [29] and [348, Lemma 4.9, p. 196].

Since we can take Siegel sets that are open to construct a rough fundamental domain for arithmetic groups in Proposition 4.34, the Siegel finiteness condition implies the following result.

Corollary 4.40. Arithmetic subgroups are finitely generated.

For special arithmetic subgroups such as $\mathrm{SL}(n, \mathbb{Z})$, more explicit generators are also known. See [386] and [395] for references.

To prove finite presentation of arithmetic groups, we need another general fact.

Proposition 4.41. Assume $X$ is a connected and simply connected locally path connected topological space (for example a simply connected manifold or a simply connected locally finite $C W$-complex), and that some $\Gamma$-action on $X$ admits a rough open fundamental domain $\Omega$ that contains only finitely many connected components. If the set $S=\{\gamma \in \gamma \Omega \cap \Omega \neq \emptyset\}$ is finite, then $\Gamma$ is finitely presented. In fact, relations between generators are given by local ones in the following sense: given any three elements $\gamma_{1}, \gamma_{2}, \gamma_{3} \in S$, the relation $\gamma_{1} \gamma_{2}=\gamma_{3}$ holds if and only if $\gamma_{1} \gamma_{2}$ and $\gamma_{3}$ induce the same action on $\Omega$, and these are all the relations needed to present $\Gamma$.

See [348, p. 196-198] for a proof. Since the symmetric space $X=G / K$ is simply connected and we can pick Siegel sets to be open and connected, we obtain the following result.

Corollary 4.42. Every arithmetic subgroup is finitely presented. 
There is also a related important notion of bounded generation. A group is called boundedly generated if there is a finite generating set $S=\left\{\gamma_{1}, \cdots, \gamma_{k}\right\}$ such that every element $\gamma$ is of the form $\gamma_{1}^{m_{1}} \cdots \gamma_{k}^{m_{k}}$, where $m_{1}, \cdots, m_{k} \in \mathbb{Z}$.

The arithmetic group $\mathrm{SL}(n, \mathbb{Z}), n \geq 3$, and more general integral subgroups of Chevalley groups of higher rank are boundedly generated. See [401].

On the other hand, if the $\mathbb{R}$-rank of $\mathbf{G}$ is equal to 1 , then $\Gamma$ is not boundedly generated. See [141]. We note that under the $\mathbb{R}$-rank 1 assumption, if $\Gamma$ is a uniform arithmetic subgroup, then $\Gamma$ is a hyperbolic group. It is also known that any non-elementary hyperbolic group is not boundedly generated.

Bounded generation is closely related to the congruence subgroup problem. For example, a special case of a theorem states that if $\mathbf{G} \subset G L(n, \mathbb{C})$ is an absolutely simple simply connected algebraic group over the rational number field $\mathbb{Q}$ and if normal subgroups of $\mathbf{G}(\mathbb{Q})$ have the standard description, ${ }^{14}$ then bounded generation of $\mathbf{G}(\mathbb{Z})$ implies that the congruence subgroup problem for $\mathbf{G}(\mathbb{Z})$ has a positive solution, i.e., the congruence subgroup kernel is finite. See $[354, \S 6][265$, Theorem D] [349] for the general result. See also [326] for a survey and more references in $[197$, p. 76$]$.

\subsection{Subgroups and overgroups}

Given any group $\Gamma$, a natural problem is to understand its subgroups. Two natural classes of groups are finite subgroups and subgroups of finite index.

Another immediate corollary of the reduction theory for arithmetic groups is the following finiteness result.

Proposition 4.43. Let $\Gamma$ be an arithmetic subgroup as in the previous subsection. Then there are only finitely many conjugacy classes of finite subgroups of $\Gamma$.

Proof. Since every finite subgroup of $\Gamma$ fixes a point in $X$, it has a conjugate that fixes a point in a fundamental set, which is the union of finitely many Siegel sets (Proposition 4.34). By the Siegel finiteness property, the fundamental set meets only finitely many translates of itself, and it follows that there are only finitely many conjugacy classes of finite subgroups.

For comparison, there are infinitely many subgroups of finite index of $\Gamma$, and also infinitely many finite quotient subgroups. A lot of work has been done on counting of subgroups of finite index. The following result holds.

\footnotetext{
${ }^{14}$ For a semisimple simply connected algebraic group $\mathbf{G}$ defined over $\mathbb{Q}$, we say that normal subgroups of $\mathbf{G}(\mathbb{Q})$ have the standard description if there exists a finite set $S$ of places of $\mathbb{Q}$ such that any Zariski-dense normal subgroup of $\mathbf{G}(\mathbb{Q})$ is open in $\mathbf{G}(\mathbb{Q})$ in the $S$-adic topology [348, p. 537].
} 
Proposition 4.44. Every arithmetic subgroup $\Gamma$ is residually finite, i.e., for every nontrivial element $\gamma \in \Gamma$, there exists a homomorphism to a finite group $F, \varphi: \Gamma \rightarrow F$, such that $\varphi(\gamma) \neq e$. In particular, $\Gamma$ contains infinitely many subgroups of infinite index. For every fixed $n \in \mathbb{N}$, there are only finitely many subgroups of index at most $n$.

To make the notion of residual finitess of $\Gamma$ quantitive, for every nontrivial element $\gamma \in \Gamma$, consider all finite quotients of $\Gamma$ such that the image of $\gamma$ in them are nontrivial. Then the minimal cardinality of such finite quotients as a function of the word length of $\gamma$ with respect to any fixed word metric on $\Gamma$ gives an invariant of the residual finiteness property of $\Gamma$. See [63] for the precise definition and some results for arithmetic subgroups of Chevalley groups.

Congruence subgroups provide a large number of subgroups of finite index. Specifically, for any positive integer $N$, the kernel of the homomorphism

$$
\mathrm{GL}(n, \mathbb{Z}) \rightarrow \mathrm{GL}(n, \mathbb{Z} / N \mathbb{Z})
$$

is clearly an arithmetic subgroup and called a principal congruence subgroup of level $N$ of $\mathrm{GL}(n, \mathbb{Z})$. Any arithmetic subgroup of $\mathrm{GL}(n, \mathbb{Z})$ containing a principal congruence subgroup is called a congruence subgroup. Congruence subgroups of $\mathbb{Q}$-linear algebraic groups $\mathbf{G}$ can also be defined similarly.

It is known that for any finitely generated group, there are only finitely many subgroups of any fixed index. The growth of the number of subgroups of index at most $n$ (or equal to $n$ ) has been actively studied. See the book [268] for an introduction and summary. See also [253] [17] for related questions on growth of finite dimensional representations of arithmetic groups.

Since arithmetic groups are linear, as a consequence of a famous Selberg Lemma [383], we have the following result.

Proposition 4.45. Every arithmetic subgroup $\Gamma$ is virtually torsion-free, i.e., it admits torsion-free subgroups of finite index.

In $[57, \S 16]$, a notion of neat arithmetic subgroups was introduced and it was proved there that every neat arithmetic subgroup is torsion-free and every arithmetic subgroup admits neat subgroups of finite index. One important difference is that many groups induced from neat arithmetic groups are also neat and hence torsion-free.

After discussing subgroups, a natural question is about groups that contain an arithmetic subgroup $\Gamma$, or overgroups of $\Gamma$. The following result holds [227].

Proposition 4.46. Assume that $\mathbf{G}$ is a semisimple linear algebraic group, then every arithmetic subgroup $\Gamma$ is contained in only finitely many discrete subgroups of $G$, in particular, in finitely many arithmetic subgroups of $\mathbf{G}(\mathbb{Q})$. 
This result is related to uniform lower bounds for the volume of locally symmetric spaces $\Gamma \backslash X$ for every fixed symmetric space $X$. It is also related to maximal arithmetic subgroups. See [36] and the references there.

It is a fact that if a group $\Gamma$ contains a proper subgroup of finite index $\Gamma^{\prime}$, then it also contains a normal subgroup of finite index. In fact, $\cap_{\gamma \in \Gamma} \gamma \Gamma^{\prime} \gamma^{-1}$ is clearly a normal subgroup of $\Gamma$ and contained in $\Gamma^{\prime}$. To show that it is also of finite index, consider the action of $\Gamma^{\prime}$ on the finite coset $\Gamma / \Gamma^{\prime}$. The kernel of this action, or of the homomorphism $\Gamma^{\prime} \rightarrow \operatorname{Sym}\left(\Gamma / \Gamma^{\prime}\right)$, is equal to the above intersection and hence is of finite index.

The famous normal subgroup theorem of Margulis [278] states.

Proposition 4.47. Assume that $\Gamma$ is an irreducible arithmetic subgroup of a semisimple linear algebraic group $\mathbf{G}$ of $\mathbb{R}$-rank at least 2. Then every normal subgroup of $\Gamma$ is either finite or of finite index.

This result says roughly that such an irreducible higher rank lattice is, as an abstract group, an almost simple group.

The rank 1 assumption is necessary. See [110].

\subsection{Borel density theorem}

As mentioned in the introduction, the realization of an arithmetic subgroup $\Gamma$ as a discrete subgroup of the Lie group $G=\mathbf{G}(\mathbb{R})$ is important for many questions about $\Gamma$. A natural problem is to understand relations between $\Gamma$ and $G$. If $G$ is semisimple, then $\Gamma$ is a lattice in $G$, i.e., the quotient $\Gamma \backslash G$ has finite volume with respect to any Haar measure of $G$. If $\Gamma$ is a cocompact lattice, then $\Gamma$ with any word metric is quasi-isometric to $G$. The former statement means that in terms of measure theory, $\Gamma$ is not too small, and the second means that in terms of large scale geometry, $\Gamma$ is not too small.

Since $G$ is the real locus of an algebraic group, it also admits the Zariski topology. Since the Zariski topology is much coarser than the regular topology of $G$, it is naturally expected that $\Gamma$ might not be a discrete subgroup in the Zariski topology. The Borel density theorem shows that this is indeed true.

Proposition 4.48. Assume that $\mathbf{G}$ is a connected semisimple linear algebraic group over $\mathbb{Q}$, and $G=\mathbf{G}(\mathbb{R})$ has no compact factor. Then any arithmetic subgroup $\Gamma \subset \mathbf{G}(\mathbb{Q})$ is Zariski dense in $G$.

One corollary of this result is the following result, which also shows one way how the Borel density can be used.

Corollary 4.49. Under the assumption of the above proposition, the normalizer of $\Gamma$ in $G$ is a discrete subgroup and hence contains $\Gamma$ as a subgroup of finite index. 
Proof. Let $N(\Gamma)$ be the normalizer of $\Gamma$ in $G$, and $M$ be the closure of $N(\Gamma)$ in the regular topology. Then $M$ is the real locus of an algebraic subgroup of G. The identity component $M^{0}$ of $M$ centralizes elements of $\Gamma$, and the Borel density theorem imply that it also centralizes $G$. Since $G$ is semisimple, $M^{0}$ consists of the identity element. Hence $M=N(\Gamma)$ is a discrete subgroup.

The Borel density theorem has many applications in rigidity theory of discrete subgroups of Lie groups. One basic reason is that in dealing with actions that are algebraic, the Borel density theorem allows one to pass from an arithmetic subgroup to the whole algebraic group, as the proof of the above corollary shows. See [432] for applications in rigidity properties of lattices.

There are also some results on discrete subgroups of $G$ that are Zariski dense but not lattices. See [353] [38].

\subsection{The Tits alternative and exponential growth}

Besides finite subgroups and finite index subgroups, it is also a natural question to understand other subgroups. The famous Tits alternative is the following result [407, Corollary 1].

Proposition 4.50. Every finitely generated linear group either contains a non-abelian free subgroup or a solvable subgroup of finite index.

A special case of another result in [407, p. 250] is the following.

Proposition 4.51. If $\mathbf{G}$ is a semisimple linear algebraic group defined over $\mathbb{Q}$ and if a subgroup $\Gamma \subset \mathbf{G}(\mathbb{Q})$ is Zariski dense, then $\Gamma$ contains a free nonabelian subgroup.

As a corollary of this and the Borel Density Theorem 4.48, we obtain the exponential growth of arithmetic groups.

Proposition 4.52. Assume that $\mathbf{G}$ is a semisimple linear algebraic group defined over $\mathbb{Q}$, and $\Gamma \subset \mathbf{G}(\mathbb{Q})$ is an arithmetic group, then $\Gamma$ grows exponentially.

We recall that for any finitely generated group $\Gamma$, there is a word metric $d_{S}$ associated with every finite generating set $S$. For any $R$, let $B(R, e)=\{\gamma \in$ $\left.\Gamma \mid d_{S}(\gamma, e) \leq R\right\}$ be the ball of radius $R$ with center $e$, and let $|B(R, e)|$ be the number of elements in the ball. We say that $\Gamma$ grows exponentially if $|B(R, e)|$ grows exponentially in $R$. Polynomial growth can be defined similarly. Though the word metric $d_{S}$ and $|B(R, e)|$ depends on the choice of the generating set $S$, the growth type of $\Gamma$ does not depend on the choice of $S$. The growth 
type often reflects algebraic properties of the group. For example, a famous theorem of Gromov says that a finitely generated group is virtually nilpotent if and only if it has polynomial growth.

For any finitely generated subgroup $\Gamma^{\prime} \subset \Gamma$, if a generating set $S^{\prime}$ is contained in a generating set $S$ of $\Gamma$, then it is clear from the definition that the restriction of the word metric $d_{S}$ to $\Gamma^{\prime}$ is bounded from above by $d_{S^{\prime}}$. This implies that if $\Gamma^{\prime}$ has exponential growth, then $\Gamma$ has at least exponential growth.

Proof of Proposition 4.52.

It can be checked easily that a non-abelian free group has exponential growth. The Borel density Theorem and Proposition 4.51 implies that the arithmetic subgroup $\Gamma$ has at least exponential growth. By some general results [106, p. 181, Remark 53 (iii)], it grows exponentially.

Remark 4.53. Another way to show that the arithmetic subgroup $\Gamma$ in Proposition 4.52 has at most exponential growth is to use the growth of the symmetric space $X$. We note that if $\Gamma$ is torsion-free and identified with an orbit $\Gamma x$ in $X$, then the induced distance on $\Gamma x$ from the invariant metric on $X$ is bounded from above by a multiple of the word metric on $\Gamma$. Since the volume of balls in $X$ grows exponentially, it follows that $\Gamma x$ with the induced metric grows exponentially and hence $\Gamma$ also grows exponentially.

\subsection{Ends of groups and locally symmetric spaces}

If $\Gamma$ is a cocompact subgroup of $G$, i.e., if the quotient $\Gamma \backslash G$ (or equivalently $\Gamma \backslash X$, where $X=G / K)$ is compact, then the number of ends of the group $\Gamma$ is the same as the number of ends of $X$, which is equal to 1 . On the other hand, if $\Gamma$ is not a cocompact subgroup, then the situation is different.

It is known that every infinite group has either 1, 2 or infinitely many ends. (See [380].) Since a group has two ends if and only if it is infinite and virtually cyclic, an arithmetic subgroup of a semisimple Lie group has either 1 or infinitely many ends.

Proposition 4.54. If $\Gamma$ is an irreducible lattice of a semisimple Lie group $G$ and the rank of the associated symmetric space $X=G / K$ is at least 2, then $\Gamma$ has one end.

Proof. Since $\Gamma$ is irreducible and the rank of $X$ is at least 2, $\Gamma$ has Property T and hence also Serre's Property FA (see [416], and also [279]). If $\Gamma$ has infinitely many ends, then a theorem Stalling [394] implies that $\Gamma$ is an amalgam (i.e., a free product with amalgamation over finite groups) and hence by Bass-Serre theory, $\Gamma$ acts on a tree without a fixed point. This contradicts with Property FA. 
It is clear that the symmetric space $X$ has one end. On the other hand, the number of ends of $\Gamma \backslash X$ depends on the $\mathbb{Q}$-rank. For example, the following result is true.

Proposition 4.55. If the $\mathbb{Q}$-rank of $\mathbf{G}$ (or rather $\Gamma \backslash X, \Gamma$ ) is greater than or equal to 2, then $\Gamma \backslash X$ has one end, i.e., it is connected at infinity. If the $\mathbb{Q}$ rank of $\Gamma$ is equal to one, then the ends of $\Gamma \backslash X$ are parametrized by the set of $\Gamma$-conjugacy classes of $\mathbb{Q}$-parabolic subgroups. By passing to smaller subgroups of finite index, there exist $\Gamma$ such that $\Gamma \backslash X$ has as many ends as desired.

The basic reason for which this proposition is true is that the $\mathbb{Q}$-Tits building $\Delta_{\mathbb{Q}}(\mathbf{G})$ is connected if and only if the $\mathbb{Q}$-rank of $\mathbf{G}$ is greater than or equal to 2. This follows from these two facts: (1) any two simplices in the Tits building are contained in a common apartment, (2) an apartment is connected if and only if the $\mathbb{Q}$-rank is greater than or equal to 2. Given this fact, Proposition 4.55 can be proved roughly as follows. By reduction theory (Proposition 4.34), the neighborhoods of infinity of $\Gamma \backslash X$ are described by Siegel sets. We can choose Siegel sets to be connected. For two $\mathbb{Q}$-parabolic subgroups $\mathbf{P}_{1}, \mathbf{P}_{2}$, the following facts can be proved: If $\mathbf{P}_{1} \subseteq \mathbf{P}_{2}$, then a Siegel set of $\mathbf{P}_{1}$ contains a Siegel set of $\mathbf{P}_{2}$. Suppose that $\mathbf{P}_{1} \cap \mathbf{P}_{2}$ is a $\mathbb{Q}$-parabolic subgroup. Then the intersection of Siegel sets of $\mathbf{P}_{1}$ and $\mathbf{P}_{2}$ is contained in a Siegel set of $\mathbf{P}_{1} \cap \mathbf{P}_{2}$. The above intersection pattern of the Siegel sets and the connectedness of the $\mathbb{Q}$-Tits building $\Delta_{\mathbb{Q}}(\mathbf{G})$ imply that $\Gamma \backslash X$ is connected at infinity. On the other hand, if the $\mathbb{Q}$-rank of $\mathbf{G}$ is equal to 1 , it can be shown that if $\mathbf{P}_{1} \neq \mathbf{P}_{2}$, then sufficiently small Siegel sets of $\mathbf{P}_{1}$ and $\mathbf{P}_{2}$ are disjoint. If $\mathbf{P}_{1}$ and $\mathbf{P}_{2}$ are not conjugate under $\Gamma$, then the image of suitable small Siegel sets of $\mathbf{P}_{1}$ and $\mathbf{P}_{2}$ in $\Gamma \backslash X$ are disjoint but are neighborhoods of the ends of $\Gamma \backslash X$.

The above argument is basically clear and convincing. To make it rigorous, it is easier to use the Borel-Serre compactification of $\Gamma \backslash X$ defined in $\S 4.18$. See [204] for a complete proof of Proposition 4.55.

\subsection{Compactifications and boundaries of symmetric spaces}

As mentioned earlier, if $G$ is semisimple, then $X=G / K$ is a symmetric space of noncompact type and consequently is noncompact. For many applications, it is important to compactify $X$ such that the $G$-action on $X$ extends continuously to the compactification.

There are many different compactifications with different boundary structures that are suitable for various applications. See the books [162] and [61] for a detailed discussion about compactifications of symmetric spaces and references.

We recall several basic facts and use them to motivate the following facts: 
(1) Points at infinity of the symmetric space $X$ are naturally described by parabolic subgroups $P$ of $G$. For example, the Furstenberg boundaries $G / P$ appear in several different compactifications of $X$.

(2) Structures at infinity of $X$ are related to an infinite simplicial complex, the Tits building $\Delta(G)$ of $G$.

We hope that this discussion will help explain similarities between Tits buildings and the curve complex $\mathcal{C}\left(S_{g, n}\right)$ of surfaces $S_{g, n}$ introduced later, which has played a foundational role in the study of mapping class groups and Teichmüller spaces (see [307] [210] summaries of recent results on applications of the curve complexes and references). For applications of Tits buildings in geometry and topology, see [208] and the extensive references there.

It is known that the symmetric space $X$ is simply connected and nonpositively curved, i.e., it is a Hadamard manifold. Therefore, $X$ admits the geodesic compactification $X \cup X(\infty)$, where $X(\infty)$ is the set of equivalence classes of directed geodesics of $X$ and is called the sphere at infinity. Since any parametrization of a geodesic is of constant speed and can be scaled to have unit speed, we assume that geodesics are directed and of unit speed. Recall that two unit speed directed geodesics $\gamma_{1}(t), \gamma_{2}(t)$ are called equivalent if $^{15}$

$$
\limsup _{t \rightarrow+\infty} d\left(\gamma_{1}(t), \gamma_{2}(t)\right)<+\infty
$$

For any basepoint $x_{0} \in X$, let $T_{x_{0}} X$ be the tangent space of $X$ at $x_{0}$. Then $X(\infty)$ can be canonically identified with the unit speed sphere in $T_{x_{0}} X$, since each equivalence class of geodesics contains exactly one unit directed geodesic through $x_{0}$. For each unit vector $v \in T_{x_{0}} X$, denote the corresponding geodesic passing through $x_{0}$ with direction $v$ by $\gamma_{v}$. Then the topology of the compactification $X \cup X(\infty)$ is described as follows: a sequence of points $x_{j} \in X$ going to infinity converges to the equivalence of $\gamma_{v}$ if and only if the direction of the geodesic segment $\overline{x_{0} x_{j}}$ converges to $v$.

It can be shown that this topology does not depend on the choice of the basepoint $x_{0}$ and the natural action of $G$ on geodesics of $X$ and hence on $X(\infty)$ induces a continuous action on the geodesic compactification $X \cup X(\infty)$.

Proposition 4.56. For every boundary point $z \in X(\infty)$, its stabilizer $G_{z}=$ $\{g \in G \mid g z=z\}$ is a proper parabolic subgroup of $G$. Furthermore, every proper parabolic subgroup of $G$ fixes some boundary point in $X(\infty)$; in fact, the subgroup is equal to the stabilizer of some boundary point.

\footnotetext{
${ }^{15}$ The assumption of the unit speed of geodesics is convenient in defining this equivalence relation. Otherwise, we need to use $d\left(\gamma_{1}(t), \gamma_{2}\right)=\inf d\left(\gamma_{1}(t), \gamma_{2}(s) \mid s \in R\right\}$, since for two equivalent geodesics $\gamma_{1}(t), \gamma_{2}(t)$ of different constant speeds, $d\left(\gamma_{1}(t), \gamma_{2}(t)\right) \rightarrow+\infty$ as $t \rightarrow+\infty$.
} 
When $X=\mathrm{SL}(2, \mathbb{R}) / \mathrm{SO}(2)$ is identified with the Poincaré upper half plane $\mathbf{H}^{2}$, then the standard parabolic subgroup $P_{\infty}$ of upper triangular matrices in $\mathrm{SL}(2, \mathbb{R})$ is the stabilizer of the boundary point $i \infty$, and the parabolic subgroup $P_{0}$ of lower triangular matrices in $\mathrm{SL}(2, \mathbb{R})$ is the stabilizer of the boundary point $0 \in \mathbb{R}$.

In this example, $X(\infty)=\mathbf{H}^{2}(\infty)=\mathbb{R} \cup\{i \infty\}=S^{1}$, and $\operatorname{SL}(2, \mathbb{R})$ acts transitively on $X(\infty)$, which can be written as $G / P_{\infty}$.

Proposition 4.57. The group $G$ acts transitively on the sphere at infinity $X(\infty)$ if and only if the rank of $X$ is equal to one. If the rank of $X$ is at least 2, then there are infinitely many $G$-orbits in the boundary $X(\infty)$, and each orbit is of the form $G / P$, where $P$ is a proper parabolic subgroup of $G$.

Probably the simplest example of higher rank symmetric spaces is the product $\mathbf{H}^{2} \times \mathbf{H}^{2}$. A maximal flat subspace of $X=\mathbf{H}^{2} \times \mathbf{H}^{2}$ can be identified with $\mathbb{R}^{2}$, and the decomposition into four coordinate quadrants corresponds to the Weyl chamber decomposition. The set of unit vectors in a positive closed Weyl chamber, say, the first quadrant, is a 1-simplex, and it parametrizes the set of $G=\mathrm{SL}(2, \mathbb{R}) \times \mathrm{SL}(2, \mathbb{R})$-orbits in $X(\infty)$.

Proposition 4.58. For a general symmetric space $X=G / K$, the set of $G$ orbits in $X(\infty)$ is parametrized by the set of unit vectors in a positive closed Weyl chamber of a maximal flat subspace of $X$, which is an $(r-1)$-simplex, where $r$ is the rank of $X$.

The homogeneous spaces $G / P$ in Proposition 4.57 are called Furstenberg boundaries. When $P$ is a minimal parabolic subgroup, $G / P$ is called the maximal Furstenberg boundary and it has played a fundamental role in the rigidity theory of lattices of $G$. See [432] and [279] for details.

For every parabolic subgroup $P$ of $G$, let $\sigma_{P}$ be the set of points of $X(\infty)$ that are fixed by $P$. Let $\sigma_{P}^{0}$ be the set of points of $X(\infty)$ whose stabilizers are exactly equal to $P$.

Proposition 4.59. For every parabolic subgroup $P$, the closure of $\sigma_{P}^{0}$ in $X(\infty)$ is equal to $\sigma_{P}$, and $\sigma_{P}$ is a simplex. Furthermore, $\sigma_{P}^{0}$ is the interior of $\sigma_{P}$ when all its boundary faces are removed. When $P$ runs over all proper parabolic subgroups of $G$, the subsets $\sigma_{P}^{0}$ give a disjoint decomposition of $X(\infty)$. The simplices $\sigma_{P}$ give the geodesic sphere $X(\infty)$ the structure of an infinite simplicial complex, which is a geometric realization of the Tits building $\Delta(G)$.

Recall that the Tits building $\Delta(G)$ is an infinite simplicial complex whose simplices are parametrized by proper parabolic subgroups of $G$ satisfying the following conditions: 
(1) For every parabolic subgroup $P$ of $G$, denote its simplex by $\sigma_{P}$. Then $P_{1} \subset P_{2}$ if and only if $\sigma_{P_{1}}$ contains $\sigma_{P_{2}}$ as a face.

(2) $\Sigma_{P}$ is a 0 -simplex (i.e., a point) if and only if $P$ is a maximal proper parabolic subgroup of $G$.

Since $G$ acts on the set of parabolic subgroups by conjugation, it acts on $\Delta(G)$ simplicially. The quotient $G \backslash \Delta(G)$ can be identified with $\sigma_{P}$ for a minimal parabolic subgroup $P$ of $G$. This result is consistent with Propositions 4.59 and 4.58 .

Besides the geodesic compactification $X \cup X(\infty)$, another important compactification is the maximal Satake compactification $\bar{X}_{\max }^{S}$.

For every real parabolic subgroup $P$, there is an $\mathbb{R}$-Langlands decomposition

$$
P=N_{P} A_{P} M_{P} \cong N_{P} \times A_{P} \times M_{P},
$$

with respect to any basepoint $x_{0}$. The dependence on the basepoint $x_{0}$ is that $A_{P}$ and $M_{P}$ are stable under the Cartan involution of $G$ associated with $K$.

Remark 4.60. When $P$ is the real locus of a $\mathbb{Q}$-parabolic subgroup $\mathbf{P}$, we have introduced a $\mathbb{Q}$-Langlands decomposition of $P$ in $\S 4.9$. The difference between these two decompositions is that in the $\mathbb{Q}$-Langlands decomposition, $A_{\mathbf{P}}$ is a maximal $\mathbb{Q}$-split component of $P$, but in the $\mathbb{R}$-decomposition here, $A_{P}$ is a maximal $\mathbb{R}$-split component of $P$. In general $A_{\mathbf{P}} \subset A_{P}$.

Define a boundary symmetric space $X_{P}$ associated with the real parabolic subgroup $P$ by

$$
X_{P}=M_{P} /\left(M_{P} \cap K\right) .
$$

Unlike the boundary symmetric space $X_{\mathbf{P}}$ for a $\mathbb{Q}$-parabolic subgroup $\mathbf{P}$ (or rather its real locus $P$ defined in $\S 4.9), X_{P}$ is always a symmetric space of noncompact type. On the other hand, $X_{\mathbf{P}}$ is equal to $X_{P}$ times a possible Euclidean factor.

Proposition 4.61. The maximal Satake compactification $\bar{X}_{\max }^{S}$ admits a disjoint decomposition

$$
\bar{X}_{\max }^{S}=X \cup \coprod_{P} X_{P}
$$

It is a compact Hausdorff space on which $G$ acts continuously.

If $X=\mathbf{H}^{2} \times \mathbf{H}^{2}$, then $\bar{X}_{\max }^{S}=\overline{\mathbf{H}^{2}} \times \overline{\mathbf{H}^{2}}$, where $\overline{\mathbf{H}^{2}}=\mathbf{H}^{2} \cup \mathbf{H}^{2}(\infty)$ is the geodesic compactification. Its boundary symmetric spaces consist of either points or $\mathbf{H}^{2}$. 
Proposition 4.62. There are finitely many $G$-orbits in the boundary $\bar{X}_{\max }^{S}-$ $X$, which are parametrized by $G$-conjugacy classes of parabolic subgroups, or standard parabolic subgroups containing a minimal parabolic subgroup $P_{0}$ of $G$. There is a unique closed orbit, which can be identified with the maximal Furstenberg boundary $G / P_{0}$ mentioned in the previous subsection.

\subsection{Baily-Borel compactification of locally symmetric spaces}

Compactifications of locally symmetric spaces are closely related to compactifications of symmetric spaces.

The basic ideas and steps can be seen in compactifications of $\Gamma \backslash \mathbf{H}^{2}$ briefly discussed in $\S 4.7$. When $\Gamma$ is a lattice of $\operatorname{SL}(2, \mathbb{R})$, i.e., a Fuchsian group of the first kind, we picked out $\Gamma$-rational points in the boundary $\mathbf{H}^{2}(\infty)$ and added them to $\mathbf{H}^{2}$ to form a partial compactification with the Satake topology. Then the quotient of this partial compactification by $\Gamma$ gives a compactification of $\Gamma \backslash \mathbf{H}^{2}$.

A natural generalization to compactify arithmetic locally symmetric spaces $\Gamma \backslash X$ initiated by Satake [374] is as follows:

(1) Start with a compactification $\bar{X}$ and decompose its boundary $\bar{X}-X$ into boundary components, which are usually parametrized by some real parabolic subgroups of $G$.

(2) Pick out rational boundary components, which are usually characterized by nonempty intersection with the closure of suitable fundamental sets (or Siegel sets of $\mathbb{Q}$-parabolic subgroups) and hence are associated with $\mathbb{Q}$-parabolic subgroups of $\mathbf{G}$.

(3) Attach the rational boundary components to $X$ to form a partial compactification ${ }_{\mathbb{Q}} \bar{X}^{S}$ of $X$ with a suitable topology, called the Satake topology.

(4) Show that $\Gamma$ acts continuously on the partial compactification $\overline{\mathbb{Q}}^{S}$ with a compact Hausdorff quotient $\Gamma \backslash_{\mathbb{Q}} \bar{X}^{S}$, which is a desired compactification of $\Gamma \backslash X$.

For the maximal Satake compactification $\bar{X}_{\text {max }}^{S}$, its boundary components are boundary symmetric spaces $X_{P}$. The rational boundary components are exactly $X_{P}$ when $P$ is the real locus of $\mathbb{Q}$-parabolic subgroups of $\mathbf{G}$. This procedure leads to the maximal Satake compactification of $\Gamma \backslash X$. In this construction, the reduction theory, in particular, the Siegel finiteness property is used crucially. In other cases, there are complications with Steps 2 and 4. The reason is that once rational boundary components are chosen, it is not obvious whether the extended $\Gamma$-action is continuous and the quotient is Hausdorff. 
A general arithmetic locally symmetric space $\Gamma \backslash X$ admits finitely many non-isomorphic Satake compactifications, and they are partially ordered, where one compactification $\overline{\Gamma \backslash X}^{1}$ is greater than or dominates another compactification $\overline{\Gamma \backslash X}^{2}$ if the identity map on $\Gamma \backslash X$ extends to a continuous map $\overline{\Gamma \backslash X}^{1} \rightarrow \overline{\Gamma \backslash X}^{2}$, which is automatically surjective. Besides the maximal Satake compactification, some minimal Satake compactifications are important for applications as well.

In order to motivate a new construction of the Deligne-Mumford compactification of $\mathcal{M}_{g}$ using the Bers compactification of the Teichmüller space $\mathcal{T}_{g}$ in $\S 5.12$, we outline the topological aspect of the Baily-Borel compactification [20] of Hermitian locally symmetric spaces. The Baily-Borel compactification is a minimal Satake compactification. See [61] for details and references.

Assume that $X=G / K$ is a Hermitian symmetric space of noncompact type, i.e., a symmetric space of noncompact type with a $G$-invariant complex structure. Then by a theorem of Harish-Chandra, $X$ can be embedded into $\mathbb{C}^{n}$ as a bounded symmetric domain, where $n$ is the complex dimension of $X$. The closure $\bar{X}$ of $X$ in $\mathbb{C}^{n}$ is called the Baily-Borel (or Baily-Borel-Satake) compactification.

As a subspace of $\mathbb{C}^{n}$, we can define analytic arc components of the boundary $\partial \bar{X}$. They are Hermitian symmetric spaces of smaller dimension. Unlike the maximal Satake compactification of $X$, they are not of the form $X_{P}$. Instead, they are the Hermitian part of the boundary symmetric spaces $X_{P}$ for maximal parabolic subgroups $P$ of $G$. (Note that the boundary symmetric space $X_{P}$ splits into a product of a Hermitian symmetric space and a linear symmetric space.) In the extended action of $G$ on $\bar{X}$, the stabilizer in $G$ of such a boundary component is equal to a maximal parabolic subgroup $P$.

Then the Baily-Borel compactification of a Hermitian locally symmetric space $\Gamma \backslash X$ can be constructed as follows:

(1) Decompose the boundary of the Baily-Borel compactification $\bar{X} \subset \mathbb{C}^{n}$ into analytic arc components.

(2) A boundary component is called rational if it has nonempty intersection with the closure of a Siegel set of a minimal $\mathbb{Q}$-parabolic subgroup.

(3) Form a partial compactification $\mathbb{Q}^{B B}$ of $X$ by adding the rational boundary components at infinity and impose the Satake topology on it.

(4) Show that the $\Gamma$-action on $X$ extends to a continuous action on ${ }_{\mathbb{Q}} \bar{X}^{B B}$ with a compact Hausdorff quotient.

(5) Show that the topological compactification $\Gamma \backslash_{\mathbb{Q}} \bar{X}^{B B}$ admits the structure of a normal complex space by constructing a sheaf of holomorphic functions on it. 
(6) Show that the normal complex space $\Gamma \backslash_{\mathbb{Q}} \bar{X}^{B B}$ is a normal projective space by embedding it into some $\mathbb{C} P^{n}$ using Poincaré-Eisenstein series.

An important new feature in this case is that the boundary components have an intrinsic interpretation in terms of the analytic structure.

\subsection{Borel-Serre compactification of locally symmetric spaces and cohomological properties of arithmetic groups}

For applications to understand topological properties of $\Gamma$, the Borel-Serre compactification of $\Gamma \backslash X[62]$ is sometimes more useful than the Satake compactifications. For example, for any Satake compactification $\overline{\Gamma \backslash X}^{S}$, the inclusion $\Gamma \backslash X \rightarrow \overline{\Gamma \backslash X}^{S}$ is not a homotopy equivalence. The fundamental group of $\overline{\Gamma \backslash X}^{S}$ is not equal to $\Gamma$. For example, when $\Gamma$ is irreducible and the rank of $X$ is at least 2 , then the fundamental group is finite. It is also trivial in some cases. (See [211] and references there.)

The importance of the Borel-Serre compactifcation is that it preserves the homotopy type of the locally symmetric space $\Gamma \backslash X$. The basic idea is to avoid Step 1 in the procedure of Satake compactifications in $\S 4.17$ since there may not exist a compactification of $X$ whose rational boundary components give rise to the desired partial compactification of $X$.

The procedure of the Borel-Serre compactification, as slightly reformulated in [61], is as follows:

(1) For every $\mathbb{Q}$-parabolic subgroup $\mathbf{P}$, define its boundary component to be $e(\mathbf{P})=N_{\mathbf{P}} \times X_{\mathbf{P}}$.

(2) Attach $e(\mathbf{P})$ to the infinity of $X$ using the horospherical decomposition of $X$ with respect to $\mathbf{P}$ to obtain a partial Borel-Serre compactification ${ }_{\mathbb{Q}} \bar{X}^{B S}$. The topology of the partial compactifcation is naturally determined by such a gluing procedure and the inductive step that if $\mathbf{P}_{1} \subset \mathbf{P}_{2}$, then $e\left(\mathbf{P}_{1}\right)$ is contained in the closure of $e\left(\mathbf{P}_{2}\right)$.

(3) Show that the $\Gamma$-action on $X$ extends to a continuous and proper action on $\overline{\mathbb{Q}}^{B S}$ with a compact quotient, which is the Borel-Serre compactification of $\Gamma \backslash X$ and denoted by $\overline{\Gamma \backslash X}^{B S}$.

In the case of $X=\mathrm{SL}(2, \mathbb{R}) / \mathrm{SO}(2)$, for every $\mathbb{Q}$-parabolic subgroup $\mathbf{P}$, its boundary component $e(\mathbf{P})=N_{\mathbf{P}} \cong \mathbb{R}$, and the partial compactification ${ }_{\mathbb{Q}} \overline{\mathbf{H}}^{B S}$ is obtained by blowing up every rational boundary point (or $\Gamma$-rational point in the sense for Fuchsian groups) into $\mathbb{R}$, and the resulting Borel-Serre compactification of $\Gamma \backslash \mathbf{H}^{2}$ has a boundary circle for every cusp end of $\Gamma \backslash \mathbf{H}^{2}$, as explained earlier.

We would like to point out that the reduction theory in Proposition 4.34 is used crucially in the above construction. For example, the Siegel finiteness 
condition is used to show that the action of $\Gamma$ on $\mathbb{Q}^{B S}$ is proper, and the fact that a union of finitely many Siegel sets form a fundamental set implies that the quotient $\Gamma \backslash_{\mathbb{Q}} \bar{X}^{B S}$ is compact, since the closure of each Siegel set in ${ }_{\mathbb{Q}} \bar{X}^{B S}$ is compact.

We now recall several basic facts about the Borel-Serre compactification and applications.

Proposition 4.63. The partial Borel-Serre compactification $\mathbb{Q}_{\mathbb{X}} \bar{X}^{B S}$ is a real analytic manifold with corners whose interior is equal to $X$. Consequently, it is contractible. The extended $\Gamma$-action on it is real analytic.

Corollary 4.64. If $\Gamma$ is torsion-free, then $\overline{\Gamma \backslash X}^{B S}$ is a compact real analytic manifold with corners and hence gives a finite model of $B \Gamma$, i.e., a model given by a finite $C W$-complex.

For the last statement, we use the general fact that a smooth compact manifold with corners admits a finite triangulation.

For the application to the virtual duality properties of $\Gamma$, we need the following theorem of Solomon-Tits.

Proposition 4.65. Let $r_{\mathbb{Q}}$ be the $\mathbb{Q}$-rank of $\mathbf{G}$. Then the Tits building $\Delta_{\mathbb{Q}}(\mathbf{G})$ is homotopy equivalent to a bouquet of infinitely many spheres $S^{r_{\mathbb{Q}}^{-1}}$ of dimension $r_{\mathbb{Q}}-1$.

Proposition 4.66. The boundary of $\overline{\mathbb{X}}^{B S}$ is homotopy equivalent to the $\mathbb{Q}$ Tits building $\Delta_{\mathbb{Q}}(\mathbf{G})$ and hence is homotopy equivalent to a bouquet of infinitely many spheres $S^{r_{\mathbb{Q}}^{-1}}$.

Recall that a group $\Gamma$ is called a Poincaré duality group of dimension $d$ if for every $\mathbb{Z} \Gamma$-module $A$, there exists an isomorphism

$$
H^{i}(\Gamma, A) \cong H_{d-i}(\Gamma, A),
$$

for all $i$. This is motivated by the Poincaré duality for closed manifolds. In fact, if $\Gamma$ admits a model of $B \Gamma$ by a closed manifold, which is necessarily an aspherical manifold, then $\Gamma$ is a Poincaré duality group.

More generally, a group $\Gamma$ is called a duality group (or a generalized Poincaré duality group) of dimension $d$ if there exists a $\mathbb{Z} \Gamma$-module $D$ such that for every $\mathbb{Z} \Gamma$-module $A$, there exists an isomorphism

$$
H^{i}(\Gamma, A) \cong H_{d-i}(\Gamma, D \otimes A),
$$

for all $i$. In this case, $D$ is called the dualizing module of $\Gamma$, and the cohomological dimension of $\Gamma$ is equal to $d$. See [79, Chap IIIV, $\S 10]$ for the history and various results on duality groups. 
It follows from the general theory that a duality group is torsion-free. A group is called a virtual duality group if it admits a finite index torsion-free subgroup that is a duality group. Virtual Poincaré duality groups can be defined similarly.

Corollary 4.67. Assume that $\Gamma$ is a torsion-free arithmetic subgroup of $\mathbf{G}(\mathbb{Q})$. Then $\Gamma$ is a duality group of dimension $\operatorname{dim} X-r_{\mathbb{Q}}$, and the dualizing module is equal to $H_{r_{\mathbb{Q}}-1}\left(\Delta_{\mathbb{Q}}(\mathbf{G}), \mathbb{Z}\right)$. Consequently, $\Gamma$ is a Poincaré duality group if and only if $r_{\mathbb{Q}}$ is equal to 0, i.e., the quotient $\Gamma \backslash X$ is compact.

The idea of the proof is as follows. Since $\mathbb{Q}^{B S}$ is contractible, it is a model of cofinite $E \Gamma$-space. By the general theory of cohomology of groups, it suffices to show that $H^{i}(\Gamma, \mathbb{Z} \Gamma)=H_{c}^{i}\left({ }_{\mathbb{Q}} \bar{X}^{B S}, \mathbb{Z}\right)$ is not equal to zero in only one degree. Then this degree is the cohomological dimension of $\Gamma$ and this $\mathbb{Z} \Gamma$-module is the dualizing module. By the Poincaré-Lefschetz duality for noncompact manifolds with corners, we have the following equalities:

$$
H_{c}^{i}\left(\mathbb{Q}^{B S}, \mathbb{Z}\right) \cong H_{n-i}\left(\mathbb{Q}^{B S} \bar{X}^{B S}, \partial_{\mathbb{Q}} \bar{X}^{B S}, \mathbb{Z}\right)=H_{n-i-1}\left(\partial_{\mathbb{Q}} \bar{X}^{B S}, \mathbb{Z}\right),
$$

where $n=\operatorname{dim} X$. By the Solomon-Tits Theorem (Proposition 4.65), the last group is zero if and only if $i \neq n-r_{Q}$, and furthermore, if $r_{\mathbb{Q}}$ is positive, then $H_{r_{\mathbb{Q}}-1}\left(\partial_{\mathbb{Q}} \bar{X}^{B S}, \mathbb{Z}\right)$ is an infinitely generated abelian group. This implies that $\Gamma$ is a duality group of dimension $\operatorname{dim} X-r_{\mathbb{Q}}$, and is a Poincaré duality group if and only if $r_{\mathbb{Q}}=0$.

\subsection{The universal spaces $E \Gamma$ and $\underline{E} \Gamma$ via the Borel-Serre partial compactification}

When $\Gamma$ is torsion-free, $\Gamma \backslash_{\mathbb{Q}} \bar{X}^{B S}$ is a finite model of $B \Gamma$ and hence ${ }_{\mathbb{Q}} \bar{X}^{B S}$ is a cofinite $E \Gamma$-space, which is also an $\underline{E} \Gamma$-space.

But most natural arithmetic subgroups such as $\operatorname{SL}(n, \mathbb{Z})$ and $\operatorname{Sp}(n, \mathbb{Z})$ are not torsion-free. As explained in the introduction, a natural question is whether arithmetic groups $\Gamma$ that contain torsion-elements admit cofinite $\underline{E} \Gamma$-spaces.

First we note the following result.

Proposition 4.68. For any arithmetic subgroup $\Gamma$, the symmetric space $X$ is a model of $\underline{E} \Gamma$.

Proof. Since $\Gamma$ acts properly on $X$, we only need to check that for any finite subgroup $F$ of $\Gamma$, the set of fixed points $X^{F}$ is nonempty and contractible. By the Cartan fixed point theorem (Proposition 4.18), $X^{F} \neq \emptyset$. Since $F$ acts by isometries, $X^{F}$ is a totally geodesic submanifold. Since $X$ is 
simply connected and nonpositively curved, $X^{F}$ is also simply connected and nonpositively curved and hence contractible.

If the quotient $\Gamma \backslash X$ is compact, then $X$ is a cofinite $\underline{E} \Gamma$-space. On the other hand, if $\Gamma \backslash X$ is noncompact, then $X$ is not a cofinite $E \Gamma$-space. Since $\Gamma \backslash_{\mathbb{Q}} \bar{X}^{B S}$ is compact, a natural guess is that ${ }_{\mathbb{Q}} \bar{X}^{B S}$ is a cofinite $\underline{E} \Gamma$-space. It is indeed true [206].

Proposition 4.69. For any arithmetic subgroup $\Gamma$, the partial Borel-Serre compactification $\overline{\mathbb{Q}}^{B S}$ is a cofinite $\underline{E} \Gamma$-space.

Since any finite subgroup $F$ of $\Gamma$ has some fixed point in $X$, we only need to show that the fixed point set $\left(\mathbb{Q}^{B S} \bar{X}^{B S}\right.$ is contractible. This can be proved by using the fact that the stabilizers in $\Gamma$ of boundary points of ${ }_{\mathbb{Q}} \bar{X}^{B S}$ are contained in the corresponding parabolic subgroups.

In the above approach, to get a cofinite $\underline{E} \Gamma$-space, we enlarge the space $X$ by adding some boundary points to get a partial compactification ${ }_{\mathbb{Q}} \bar{X}^{B S}$ so that the quotient $\Gamma \backslash_{\mathbb{Q}} \bar{X}^{B S}$ becomes compact. One important requirement on the compactification is that the inclusion $X \hookrightarrow \mathbb{Q}^{B S}$ is a $\Gamma$-equivariant homotopy equivalence.

Another way to overcome the noncompactness is to take a subspace $S$ of $X$ such that

(1) $S$ admits the structure of a $\Gamma$-CW complex such that $\Gamma \backslash S$ is compact.

(2) $S$ is a $\Gamma$-equivariant deformation retract of $X$.

Then it can be checked easily that $S$ is also an $\underline{E} \Gamma$-space and hence is a cofinite model of $E \Gamma$. This is the problem of existence of a good equivariant spine in $X$.

If $X$ is the upper half plane $\mathbf{H}^{2}$ and $\Gamma$ is commensurable with $\operatorname{SL}(2, \mathbb{Z})$, by removing small horodisks around the rational boundary points in a $\Gamma$ equivariant way, we obtain a $\Gamma$-stable truncated subspace $X(\varepsilon)$, which is a submanifold with boundary, where $\varepsilon$ measures the sizes of the horodisks. It can be shown that the above two conditions are satisfied by $X(\varepsilon)$.

It turns out that if we push these horodisks further until they meet and flatten out, the subspace $X(\varepsilon)$ becomes a tree $T$ which is stable under $\Gamma$. (See [384].) What is important is that $\operatorname{dim} T$ is equal to the virtual cohomological dimension of $\Gamma$, which is the optimal dimension.

The above truncation procedure of removing horoballs equivariantly from $\mathbf{H}^{2}$ can be generalized to a general symmetric space $X$ by removing horoballs in $X$ associated with $\mathbb{Q}$-parabolic subgroups which are equivariant with respect to an arithmetic subgroup $\Gamma$. Then the remaining subspace is a manifold with corners and is stable under $\Gamma$. Denote it by $X_{T}$. Then the quotient $\Gamma \backslash X_{T}$ is 
a compact manifold with corners. (It might be worthwhile to point out that if the rank of $X$ is greater than or equal to 2 , then the equivariant horoballs above intersect each other no matter how small the horoballs are.) This is related to $\Gamma$-equivariant tilings of $X$ mentioned before in $\S 4.10$. The central tile in [371] is a cocompact deformation retract of $X$ and is equal to $X_{T}$, which corresponds to $X(\varepsilon)$ in the case of the upper half plane $\mathbf{H}^{2}$ above. This gives another cofinite model of $\underline{E} \Gamma$ different from the one in Proposition 4.69.

As in the case of the upper half plane $\mathbf{H}^{2}$, a natural and important question is whether for a non-uniform arithmetic subgroup $\Gamma$, the symmetric space $X$ admits a $\Gamma$-equivariant deformation retraction $S$ such that $\Gamma \backslash S$ is compact and $\operatorname{dim} S$ is equal to the virtual cohomological dimension of $\Gamma$.

This seems to be a difficult problem and the answer is not known in general. The following is a list of cases where the answer is positive.

(1) When $X$ is a linear symmetric space.

(2) When the $\mathbb{Q}$-rank of $\Gamma \backslash X$ is equal to 1 .

(3) When the $\mathbb{R}$-rank of $X$ is equal to 1 .

(4) When $\Gamma=\operatorname{Sp}(4, \mathbb{Z})$ and $X$ is the Siegel upper space of degree 2 .

See the papers [14] [281] [428] and references there. In a work in progress, we are able to find such an equivariant spine when the $\mathbb{Q}$-rank of $\Gamma \backslash X$ is less than or equal to 2 .

\section{Mapping class groups $\operatorname{Mod}_{g, n}$}

In this section, we introduce some definitions and results about Teichmüller spaces $\mathcal{T}_{g, n}$ and mapping class groups $\operatorname{Mod}_{g, n}$ by emphasizing their similarities to symmetric spaces and arithmetic subgroups. Since some results and proofs are motivated by and related to results for symmetric and locally symmetric spaces in the previous section, they will be rather brief. For a systematic discussion about Teichmüller spaces and mapping class groups, see the first

two volumes of this handbook [334] [335]. See also the book [127] and the survey papers [188] [124] [169].

\subsection{Definitions and examples}

Let $S_{g, n}$ be an oriented surface of genus $g$ with $n$ punctures. When $n=0$, we denote it by $S_{g}$.

A natural procedure to construct new topological spaces and groups from $S_{g, n}$ is to consider the group $\mathrm{Homeo}^{+}\left(S_{g, n}\right)$ of orientation preserving homeomorphisms of $S_{g, n}$. Denote its identity component by $\operatorname{Homeo}^{0}\left(S_{g, n}\right)$. Then this 
identity component $\mathrm{Homeo}^{0}\left(S_{g, n}\right)$ is a normal subgroup of $\mathrm{Homeo}^{+}\left(S_{g, n}\right)$, and the quotient $\mathrm{Homeo}^{+}\left(S_{g, n}\right) / \mathrm{Homeo}^{0}\left(S_{g, n}\right)$ is called the mapping class group of $S_{g, n}$ and denoted by $\operatorname{Mod}_{g, n}$. Since the set of connected components of a topological space has a natural discrete topology, it seems reasonable to give $\operatorname{Mod}_{g, n}$ the discrete topology.

Equivalently, if $\operatorname{Diff}^{+}\left(S_{g, n}\right)$ denotes the group of orientation preserving diffeomorphisms of $S_{g, n}$, its identity component $\operatorname{Diff}^{0}\left(S_{g, n}\right)$ is a normal subgroup of $\operatorname{Diff}^{+}\left(S_{g, n}\right)$. Then the quotient $\operatorname{Diff}^{+}\left(S_{g, n}\right) / \operatorname{Diff}^{0}\left(S_{g, n}\right)$ is also equal to $\operatorname{Mod}_{g, n}$.

When $g=1, n=0$, it can be shown that $\operatorname{Mod}_{g, n} \cong \mathrm{SL}(2, \mathbb{Z})$. For any smooth manifold $M$, we can also define its mapping class group $\operatorname{Mod}(M)=$ $\operatorname{Diff}^{+}(M) / \operatorname{Diff}^{0}(M)$ in a similar way. It can be shown that when $M=\mathbb{R}^{n} / \mathbb{Z}^{n}$, the $n$-dimensional torus, $\operatorname{Mod}(M) \cong \mathrm{SL}(n, \mathbb{Z})$.

\subsection{Teichmüller spaces}

It is known that the surface $S_{g, n}$ admits complex structures so that each puncture admits neighborhoods that are biholomorphic to the punctured disc $D^{\times}=\{z \in \mathbb{C}|0<| z \mid<1\}$.

The moduli space of all such complex structures on $S_{g, n}$ is denoted by $\mathcal{M}_{g, n}$. It was first introduced by Riemann and has been intensively studied since then.

If $2 g-2+n>0$, then by the uniformization theorem for Riemann surfaces, each complex structure on $S_{g, n}$ is biholomorphic to $\Gamma \backslash \mathbf{H}^{2}$, where $\Gamma \subset$ $P \mathrm{SL}(2, \mathbb{R})$ is a torsion-free lattice subgroup (or rather a Fuchsian group of the first kind.) Therefore, for every complex structure on $S_{g, n}$ as above, there exists a unique complete hyperbolic metric on $S_{g, n}$ of finite total area that is conformal to the complex structure. Then $\mathcal{M}_{g, n}$ is also the moduli space of all complete hyperbolic metrics of finite total area of $S_{g, n}$.

If we identify each complex structure on $S_{g, n}$ with a projective curve over $\mathbb{C}$ with $n$ marked points, then $\mathcal{M}_{g, n}$ is also the moduli space of projective curves over $\mathbb{C}$ with $n$ marked points. This is one of the most important moduli spaces in algebraic geometry.

According to a general philosophy, the moduli space of objects with certain structures (smooth structures, complex structures, algebraic structures etc.) should inherit structures similar to those of the objects.

Based on this philosophy, we expect to have and should be able to establish:

(1) As the moduli space of complex structures on $S_{g, n}$, the moduli space $\mathcal{M}_{g, n}$ should admit a complex structure, i.e., it should be a complex space;

(2) as the moduli space of Riemann metrics of constant curvature, $\mathcal{M}_{g, n}$ should admit (Riemannian) metrics; 
(3) as the moduli space of algebraic curves over $\mathbb{C}, \mathcal{M}_{g, n}$ should admit the structure of an algebraic variety, i.e., it should be an algebraic variety.

It turns out that all these statements are true, and hence the moduli space $\mathcal{M}_{g, n}$ is the best example to show the above philosophy. In some sense, the rich structures on $\mathcal{M}_{g, n}$ make it one of the most interesting and important spaces in mathematics.

Remark 5.1. There is a theorem of Wielandt (see [366] for example) saying that for any finite group $G$ with trivial center, its automorphism tower $G \subset \operatorname{Aut}(G) \subset \operatorname{Aut}^{2}(G)=\operatorname{Aut}(\operatorname{Aut}(G)) \subset \cdots$ terminates after finitely many steps. One way to interpret this is to view $\operatorname{Aut}(G)$ as a genuinely new group constructed from $G$. The above result says that this process will terminate after finitely many steps. We can also consider the related outer automorphism groups $\operatorname{Out}(G), \operatorname{Out}^{2}(G), \cdots$. The natural generalization of Wielandt's theorem to infinite groups does not hold in general. On the other hand, for the following three classes of groups related to this chapter: the abelian free groups $\mathbb{Z}^{n}$, the non-abelian free groups $F_{n}$, and the surface groups $\pi_{1}\left(S_{g}\right)$, their automorphism towers do terminate after finitely many steps. Indeed, $\operatorname{Aut}\left(\mathbb{Z}^{n}\right)=\mathrm{GL}(n, \mathbb{Z})$, Out $\left(F_{n}\right)$, Out $\left(\pi_{1}\left(S_{g}\right)\right)=\operatorname{Mod}_{g}$ are rigid. The automorphism groups of $\mathrm{GL}(n, \mathbb{Z})$ and more generally arithmetic groups, $\operatorname{Mod}_{g, n}$ and $\operatorname{Out}\left(F_{n}\right)$ are related to Mostow strong rigidity and have been discussed in the earlier sections.

A natural question is how to construct new spaces starting from some new spaces. As discussed in the previous subsection, one way to construct new spaces is to consider the space of homeomorphisms (or diffeomorphisms). Another natural way is to consider moduli spaces of certain structures on the spaces we started with. If we mimic the automorphism tower and construct the moduli spaces inductively, a natural guess is that this process should terminate after finitely many steps, i.e., the moduli space will eventually become stationary.

This turns out to be true for Riemann surfaces (hyperbolic surfaces, or algebraic curves over $\mathbb{C}$ ), since $\mathcal{M}_{g, n}$ is rigid. If we start with the torus $\mathbb{Z}^{n} \backslash \mathbb{R}^{n}=\left(S^{1}\right)^{n}$ and consider the moduli space of flat metrics of total volume 1 , then we get $\mathrm{SL}(n, \mathbb{Z}) \backslash \mathrm{SL}(n, \mathbb{R}) / \mathrm{SO}(n)$, which is also rigid.

If we consider the moduli spaces of other objects such as $K-3$ surfaces etc, their moduli spaces are given by arithmetic locally symmetric spaces and are often rigid. See the book [185] and [207] for additional references.

In order to study $\mathcal{M}_{g}$ and put a complex structure on it, Teichmüller initiated the systematic study of the Teichmüller space $\mathcal{T}_{g}$ via quasi-conformal maps and hence introduced a metric on it. He also considered the question of complex structures on $\mathcal{T}_{g}$. On reason why it is easier to study the Teichmüller space is that in general it is easier to study moduli spaces of more rigid objects, 
i.e., those that do not admit self-automorphisms, and to put structures of smooth manifolds on the moduli spaces.

Let $S_{g, n}$ be a compact oriented surface of genus $g$ with $n$ points removed. A Riemann surface $\Sigma_{g, n}$ of genus $g$ with $n$ punctures together with a homotopy class of orientation preserving diffeomorphisms $\varphi: \Sigma_{g, n} \rightarrow S_{g, n}$ is called a marked Riemann surface and denoted by $\left(\Sigma_{g, n},[\varphi]_{g, n}\right)$. Two marked Riemann surfaces $\left(\Sigma_{g, n},[\varphi]_{g, n}\right)$ and $\left(\Sigma_{g, n}^{\prime},\left[\varphi^{\prime}\right]_{g, n}\right)$ are defined to be equivalent if there exists a biholomorphic map $h: \Sigma_{g, n} \rightarrow \Sigma_{g, n}^{\prime}$ such that $\varphi^{\prime} \circ h$ is homotopy equivalent to $\varphi$.

Then the Teichmüller space $\mathcal{T}_{g, n}$ is defined to be the set of equivalence classes of marked Riemann surfaces $\left(\Sigma_{g, n},[\varphi]_{g, n}\right)$ :

$$
\mathcal{T}_{g, n}=\left\{\left(\Sigma_{g, n},[\varphi]_{g, n}\right)\right\} / \sim .
$$

Remark 5.2. A marking on a Riemann surface $\Sigma_{g, n}$ above is equivalent to a choice of a set of generators of the fundamental group $\pi_{1}\left(\Sigma_{g, n}\right)$, but not equivalent to a choice of a basis of $H_{1}\left(\Sigma_{g, n}\right)$, which is more common in Hodge theory.

In this chapter, we assume (except if we specify the contrary) that $2 g-2+$ $n>0$. Then each Riemann surface $\Sigma_{g, n}$ admits a unique complete hyperbolic metric of finite area that is conformal to the complex structure. Under this assumption, the Teichmüller space $\mathcal{T}_{g, n}$ can also be defined to be the moduli space of marked complete metrics of finite volume on $S_{g, n}$.

As defined earlier, Diff ${ }^{+}\left(S_{g, n}\right)$ is the group of orientation preserving diffeomorphisms of $S_{g, n}$, and $\operatorname{Diff}^{0}\left(S_{g, n}\right)$ its identity component. Then the quotient group $\operatorname{Diff}^{+}\left(S_{g, n}\right) / \operatorname{Diff}^{0}\left(S_{g, n}\right)$ is the mapping class group $\operatorname{Mod}_{g, n}$.

$\operatorname{Mod}_{g, n}$ acts on $\mathcal{T}_{g, n}$ by changing the markings: for any $\psi \in \operatorname{Diff}^{+}\left(S_{g, n}\right)$ and a marked Riemann surface $\left(\Sigma_{g, n},[\varphi]\right)$,

$$
\psi \cdot\left(\Sigma_{g, n},[\varphi]\right)=\left(\Sigma_{g, n},[\psi \circ \varphi]\right) .
$$

Clearly, the quotient $\operatorname{Mod}_{g, n} \backslash \mathcal{T}_{g, n}$ is equal to the moduli spaces $\mathcal{M}_{g, n}$ of Riemann surfaces $\Sigma_{g, n}$ of genus $g$ with $n$ punctures.

Remark 5.3. The idea of Teichmüller spaces has also been used for other spaces and groups. For example, the outer spaces $X_{n}$ of marked metric graphs are defined in an almost identical way. As the earlier discussions indicated, the similarity between the action of $\operatorname{Out}\left(F_{n}\right)$ on $X_{n}$ with the action of $\operatorname{Mod}_{g, n}$ on $\mathcal{T}_{g, n}$ has inspired a lot of exciting work. See [412] [414] [45] and references there.

For general manifolds, the theory of Teichmüller spaces is often different. See [134]. 


\subsection{Properties of Teichmüller spaces}

In comparison with symmetric spaces of noncompact type, the first basic result is the following.

Proposition 5.4. The Teichmüller space $\mathcal{T}_{g, n}$ is a real analytic manifold diffeomorphic to $\mathbb{R}^{6 g-6+2 n}$, and $\operatorname{Mod}_{g, n}$ acts properly on $\mathcal{T}_{g, n}$.

It was first shown by Fricke that $\mathcal{T}_{g, n}$ has the structure of a real analytic manifold and is homeomorphic to $\mathbb{R}^{6 g-6+2 n}$. An explicit homeomorphism (or rather diffeomorphism) was given by Teichmüller via quasiconformal maps of smallest distortion. Probably the easiest way to see the diffeomorphism is to use the Fenchel-Nielsen coordinates. For details, see [327] [40, p. 269]. See also [365] for a historical summary of Teichmüller spaces.

For the purpose of constructing the Deligne-Mumford compactification of $\mathcal{M}_{g, n}$ using the Bers compactification of $\mathcal{T}_{g, n}$ in this chapter, the following result is important as well.

Proposition 5.5. $\mathcal{T}_{g, n}$ is a complex manifold of dimension $3 g-3+n$ and can be realized as a bounded domain in $\mathbb{C}^{3 g-3-n}$ under the Bers embedding. $\operatorname{Mod}_{g, n}$ acts on $\mathcal{T}_{g, n}$ by biholomorphic maps.

One way to view the complex structure of $\mathcal{T}_{g, n}$ is to take a base Riemann surface $\left(\Sigma_{g, n},[\varphi]\right)$ and consider the complex Banach space $\mathcal{B}$ of all Beltrami differentials on $\Sigma_{g, n}$, and realize $\mathcal{T}_{g, n}$ as a quotient of $\mathcal{B}$. See [327].

The tangent space of $\mathcal{T}_{g, n}$ at $\left(\Sigma_{g, n},[\varphi]\right)$ can be identified with the space of harmonic Beltrami differentials on $\Sigma_{g, n}$, and the cotangent space can be identified with the space of holomorphic quadratic differential forms on $\Sigma_{g, n}$.

For the action of $\operatorname{Mod}_{g, n}$ on $\mathcal{T}_{g, n}$, the following Nielsen realization result [233] [424] is important.

Proposition 5.6. For every finite subgroup $F \subset \operatorname{Mod}_{g, n}$, there exists a Riemann surface $\Sigma_{g, n}$ such that $F$ is contained in $\operatorname{Aut}\left(\Sigma_{g, n}\right)$ and hence the set of fixed point $\mathcal{T}_{g, n}^{F}$ is nonempty.

\subsection{Metrics on Teichmüller spaces}

For the symmetric space $X=G / K$ discussed earlier, there is a $G$-invariant Riemannian metric which is unique up to scaling. This invariant metric enjoys many good properties. It has been used effectively in many contexts and is suitable for different applications.

On the other hand, the Teichmüller space $\mathcal{T}_{g, n}$ admits many different metrics introduced for various applications. They are all natural. In some sense, 
the presence of different metrics on $\mathcal{T}_{g, n}$ makes it a more interesting space. But the lack of homogeneity of these metrics makes it more difficult to understand their properties.

We will mention two such metrics: the Teichmüller metric and the WeilPetersson metric.

Given any two marked Riemann surfaces $p_{1}=\left(\Sigma_{g, n},[\varphi]\right), p_{2}=\left(\Sigma_{g, n}^{\prime},\left[\varphi^{\prime}\right]\right)$, for any quasi-coformal map $f$ in the homotopy class $\left[\left(\varphi^{\prime}\right)^{-1} \circ \varphi\right]$ from $\Sigma_{g, n}$ to $\Sigma_{g, n}^{\prime}$, let $K(f)$ be the dilation of $f$. Then the Teichmüller distance between $p_{1}, p_{2}$ is

$$
d_{T}\left(p_{1}, p_{2}\right)=\inf _{f} \log K(f) .
$$

It is a complete Finsler metric and has the property that any two distinct points are connected by a unique geodesic. But it is not a CAT(0)-metric.

Another important metric is the Weil-Petersson metric on $\mathcal{T}_{g, n}$. At any point $\left(\Sigma_{g, n},[\varphi]\right) \in \mathcal{T}_{g, n}$, the cotangent bundle of $\mathcal{T}_{g, n}$ is equal to the space $Q\left(\Sigma_{g, n}\right)$ of holomorphic quadratic differential forms on $\Sigma_{g}$. Let $d s^{2}$ be the hyperbolic metric of $\Sigma_{g, n}$. Then the inner product on the cotangent bundle is given by: for $\varphi_{1}, \varphi_{2} \in Q\left(\Sigma_{g, n}\right)$,

$$
\left\langle\varphi_{1}, \varphi_{2}\right\rangle=\int_{\Sigma_{g, n}} \varphi_{1} \overline{\varphi_{2}}\left(d s^{2}\right)^{-1} .
$$

It is a Kähler metric but is incomplete. On the other hand, it has the following important property [425] [426].

Proposition 5.7. The Weil-Petersson metric on $\mathcal{T}_{g, n}$ is negatively curved and geodesically convex in the sense that every two points are connected by a unique geodesic.

Though the Weil-Petersson metric is not complete, the result in this proposition is a good replacement. For example, the basic Cartan fixed point theorem (Proposition 4.18) for actions of compact isometry groups on complete Riemannian manifolds of nonpositive curvature holds in this case.

\subsection{Compacfications and boundaries of Teichmüller spaces}

Since $\mathcal{T}_{g, n}$ is noncompact, a natural problem is to construct and study some natural compacfitications.

The following are few compactifications among all compactifications of $\mathcal{T}_{g, n}$ :

(1) The Teichmüller ray compactification of $\mathcal{T}_{g, n}$ obtained by identifying $\mathcal{T}_{g, n}$ with $\mathbb{R}^{6 g-6+2 n}$ using Teichmüller rays from a fixed basepoint and adding the sphere $S^{6 g-6+2 n-1}$ at infinity [235]. 
(2) The Bers compactification of $\mathcal{T}_{g, n}$ obtained by taking the closure of the image of $\mathcal{T}_{g, n}$ under the Bers embedding [327].

(3) The Thurston compactification [403].

(4) The harmonic map compactification [423].

(5) The extremal length compactification [144].

(6) The horofunction compactification with respect to the asymmetric Thurston metric [415].

(7) The real spectrum compactification of $\mathcal{T}_{g}$ [82].

(8) The compactification via actions on $\Lambda$-trees [316].

Among the eight compactifications, the harmonic map compactification, the horofunction compactification and the compactification via actions on $\mathbb{R}$ trees (i.e., when $\lambda=\mathbb{R}$ in [316]) are isomorphic to the Thurston compactification.

It is also known that the action of $\operatorname{Mod}_{g, n}$ on $\mathcal{T}_{g, n}$ extends to a continuous action to the Thurston compactification and hence also to the harmonic map compactification and the horofunction compactification. It also extends continuously to the real spectrum compactification of $\mathcal{T}_{g}$. On the other hand, it does not extend continuously to the Teichmüller ray compactification or the Bers compactification [234] [235].

The continuous extension of the action of $\operatorname{Mod}_{g, n}$ to the Thurston compactification was used crucially in the classification of elements of $\operatorname{Mod}_{g, n}$. The action of $\operatorname{Mod}_{g, n}$ on the Thurston boundary is also important for various rigidity results [165] [166] [236] [237].

It seems that the other compactifications have not been used for similar applications. On the other hand, there is a closely related partial compactification of $\mathcal{T}_{g, n}$, which can be obtained from the Bers compactification. It can be described in several different ways.

Recall that a Riemann surface $\Sigma$ is called stable if its group of biholomorphic automorphisms is finite. This is equivalent to the condition that each connected component of $\Sigma$ has negative Euler characteristic and hence it is also equivalent to the condition that $\Sigma$ admits a complete hyperbolic metric of finite area. (We note that the isometry group of any hyperbolic manifold of finite volume is finite.) For example, a compact Riemann surface $\Sigma_{g}$ of genus $g$ is stable if and only if $g>1$. More generally, $\Sigma_{g, n}$ is stable if and only if $2 g-2+n>0$.

In [1], the augmented Teichmüller spaces $\hat{\mathcal{T}}_{g, n}$ was introduced. As a set, it is the union of $\mathcal{T}_{g, n}$ and the set of stable Riemann surfaces which are obtained from $\Sigma_{g, n}$ by pinching along some simple closed geodesics. These boundary stable Riemann surfaces are also marked in some sense. More specifically, they correspond to the so-called regular $b$-groups in the boundary of the Bers compactification [42] [284] [1]. 
Three equivalent topologies were introduced on $\hat{\mathcal{T}}_{g, n}$ in [1]. They basically correspond to the intuitive idea that a sequence of marked Riemann surfaces $\Sigma_{g, n}$ converges to a boundary stable curve if and only if a collection of disjoint simple closed curves are pinched.

Its relation with the induced subspace topology from the Bers compactification was not clear or discussed in [1]. We will address this later by using the method of Satake compactifications of locally symmetric spaces.

One application of the augmented Teichmüller space $\hat{\mathcal{T}}_{g, n}$ is the following result [1].

Proposition 5.8. The action of $\operatorname{Mod}_{g, n}$ on $\mathcal{T}_{g, n}$ extends to a continuous, but not proper, action on $\hat{\mathcal{T}}_{g, n}$, and the quotient $\operatorname{Mod}_{g, n} \backslash \hat{\mathcal{T}}_{g, n}$ is a compact Hausdorff space. The compact quotient $\operatorname{Mod}_{g, n} \backslash \hat{\mathcal{T}}_{g, n}$ is a compactification of the moduli space $\mathcal{M}_{g, n}$ and equal to the Deligne-Mumford compactification.

As mentioned before, the Weil-Petersson metric is not complete. An important result is the following [291] [425] [427].

Proposition 5.9. The completion of $\mathcal{T}_{g, n}$ with respect to the Weil-Petersson metric is canonically homeomorphic to the augmented Teichmüller space $\hat{\mathcal{T}}_{g, n}$. Furthermore, with the extended distance function, $\hat{\mathcal{T}}_{g, n}$ is a CAT(0)-space.

This result is satisfying since it realizes the augmented Teichmüller space $\hat{\mathcal{T}}_{g, n}$ completely in terms of an intrinsic metric of $\mathcal{T}_{g, n}$.

One consequence is the following realization of the Deligne-Mumford compactification $\overline{\mathcal{M}}_{g, n} D M$.

Corollary 5.10. The completion of $\mathcal{M}_{g, n}$ with respect to the Weil-Petersson metric is equal to the Deligne-Mumford compactification $\overline{\mathcal{M}}_{g, n} D M$.

One consequence of this together with the results on the automorphism group of the curve complex $\mathcal{C}\left(S_{g, n}\right)$ [188] [271] is the following corollary [293] [425].

Corollary 5.11. With a few exceptions, the isometry group of the WeilPetersson metric of $\mathcal{T}_{g, n}$ is equal to $\operatorname{Mod}_{g, n}$.

\subsection{Curve complexes and boundaries of partial compactifications}

The boundary of the augmented Teichmüller space $\hat{\mathcal{T}}_{g, n}$ consists of Teichmüller spaces of Riemann surfaces of lower genus with more punctures and of the same Euler characteristic. 
The inclusion relations between these boundary components of $\hat{\mathcal{T}}_{g, n}$ can be described in terms of the curve complex $\mathcal{C}\left(S_{g, n}\right)$ of the surface $S_{g, n}$. It is an infinite simplicial complex and plays an important role for the Teichmüller space $\mathcal{T}_{g, n}$ as does the spherical Tits building $\Delta_{\mathbb{Q}}(\mathbf{G})$ for the symmetric space $X$.

Specifically, consider the collection of the homtopy classes $[c]$ of all essential simple closed curves in $S_{g, n}$, i.e., simple closed curves that are not trivial or homotopic to a loop around a puncture. They parametrize the vertices of $\mathcal{C}\left(S_{g, n}\right)$. The vertices $\left[c_{1}\right], \cdots,\left[c_{k+1}\right]$ form the vertices of a $k$-simplex if and only if they admit disjoint representatives.

It is known that $\mathcal{C}\left(S_{g, n}\right)$ is an infinite simplicial complex of infinite diameter [188]. It clear that $\operatorname{Mod}_{g, n}$ acts simplicially on $\mathcal{C}\left(S_{g, n}\right)$.

Proposition 5.12. The quotient $\operatorname{Mod}_{g, n} \backslash \mathcal{C}\left(S_{g, n}\right)$ is a finite complex.

Proof. Since a simplex of $\mathcal{C}\left(S_{g, n}\right)$ of maximal dimension corresponds to a maximal collection of disjoint non-homotopic simple closed curves on $S_{g, n}$, i.e., a pants decomposition, and since there are only finitely many homeomorphism classes of pants decompositions, the proposition follows.

An important result due to Harer [169] is the following.

Proposition 5.13. The curve complex $\mathcal{C}\left(S_{g, n}\right)$ is homotopy equivalent to a bouquet of spheres.

This is the analogue of the Solomon-Tits theorem for Tits buildings (Proposition 4.65). From the proof, it was not clear if the bouquet contains at least one sphere, i.e., if $\mathcal{C}\left(S_{g, n}\right)$ has trivial topology. This was answered in [192].

Proposition 5.14. The curve complex $\mathcal{C}\left(S_{g, n}\right)$ is homotopy equivalent to a bouquet of infinitely many spheres. The dimension $d$ of the spheres is $d=2 g-2$ when $n=0$ and $g \geq 2$, and $d=2 g-3+n$ when $g>0$ and $n>0$, and $d=n-4$ when $g=0$ and $n \geq 4$.

Due to the collar theorem, two (or more) simple closed geodesics on a hyperbolic surface $\Sigma_{g, n}$ can be pinched simultaneously if and only if they are disjoint. Then it is easy to imagine that each boundary Teichmüller space of $\hat{\mathcal{T}}_{g, n}$ corresponds to a simplex of $\mathcal{C}\left(S_{g, n}\right)$.

Proposition 5.15. For each simplex $\sigma$ of $\mathcal{C}\left(S_{g, n}\right)$, there is a boundary Teichmüller space $\mathcal{T}_{\sigma}$ of $\hat{\mathcal{T}}_{g, n}$, and for any two simplices $\sigma_{1}, \sigma_{2}$ in $\mathcal{C}\left(S_{g, n}\right), \mathcal{T}_{\sigma_{1}}$ is contained in $\mathcal{T}_{\sigma_{2}}$ as a face if and only if $\sigma_{1}$ contains $\sigma_{2}$ as a face.

Since each boundary Teichmüller space $\mathcal{T}_{\sigma}$ is contractible, we get the following result. 
Corollary 5.16. The boundary of the augmented Teichmüller space $\hat{\mathcal{T}}_{g, n}$ is connected and has the homotopy type of a bouquet of infinitely many spheres.

Remark 5.17. One way to decompose intrinsically the boundary of the augmented Teichmüller space $\hat{\mathcal{T}}_{g, n}$ into boundary Teichmüller spaces is to consider the maximal totally geodesic subspaces when $\hat{\mathcal{T}}_{g, n}$ is considered as the completion of the Weil-Petersson metric and as a CAT(0)-space. For detailed discussion of the CAT(0)-geometry of the augmented Teichmüller space $\hat{\mathcal{T}}_{g, n}$, see [425] [427].

See also [426] [427] for a detailed description of geometry of the boundary Teichmüller spaces in $\hat{\mathcal{T}}_{g, n}$.

\subsection{Universal spaces for proper actions}

As mentioned before, given any discrete group $\Gamma$, a natural problem is to construct good models of universal spaces $\underline{E} \Gamma$ for proper actions of $\Gamma$, in particular, cofinite models of $\underline{E} \Gamma$ spaces.

Proposition 5.18. The Teichmüller space $\mathcal{T}_{g, n}$ is a model of the universal spaces $\underline{E}_{M_{g, n}}$ for proper actions of $\operatorname{Mod}_{g, n}$.

Proof. For any finite subgroup $F \subset \operatorname{Mod}_{g, n}$, by the Nielsen realization result (Proposition 5.6), the set of fixed points $\mathcal{T}_{g, n}^{F}$ is nonempty. To show that it is contractible, take any two points $p, q \in \mathcal{T}_{g, n}^{F}$. Consider the Weil-Petersson geodesic connecting them. Since such a geodesic is unique and $p, q$ are fixed by $F$, the geodesic is also fixed by $F$. This implies that $\mathcal{T}_{g, n}^{F}$ is a totally geodesic subspace of $\mathcal{T}_{g, n}$ and is hence contractible.

Remark 5.19. We can also use the fact that with respect to the Teichmüller metric, every two points are connected by a unique geodesic to prove the second statement in the proof. The negative curvature of the Weil-Petersson metric can also be used to prove nonemptyness of the fixed point set $\mathcal{T}_{g, n}^{F}$.

Since $\operatorname{Mod}_{g, n} \backslash \mathcal{T}_{g, n}$ is noncompact, $\mathcal{T}_{g, n}$ is not a cofinite space of $\operatorname{Mod}_{g, n}$. To overcome this difficulty, a natural method is to construct an analogue of the Borel-Serre partial compactification of symmetric spaces. Such an construction was outlined by Harvey [172] and carried out by Ivanov (see [188] and references). On the other hand, it is not obvious that it satisfies the property that the fixed point set of any finite subgroup on the partial compactifcation is contractible.

Another way is to remove suitable neighborhoods of the infinity of $\mathcal{T}_{g, n}$ so that its quotient by $\operatorname{Mod}_{g, n}$ is compact. 
For any small constant $\varepsilon>0$, define the thick part of $\mathcal{T}_{g, n}$ by

$$
\mathcal{T}_{g, n}(\varepsilon)=\left\{\left(\Sigma_{g, n},[\varphi]\right) \in \mathcal{T}_{g, n} \mid \Sigma_{g, n} \text { has no closed geodesic of length }<\varepsilon\right\} .
$$

Proposition 5.20. For $\varepsilon$ sufficiently small, the thick part $\mathcal{T}_{g, n}(\varepsilon)$ is a real analytic manifold with corners and invariant under $\operatorname{Mod}_{g, n}$, and the quotient $\operatorname{Mod}_{g, n} \backslash \mathcal{T}_{g, n}(\varepsilon)$ is a compact real analytic manifold with corners and hence has the structure of a finite $C W$-complex.

The key statement that $\operatorname{Mod}_{g, n} \backslash \mathcal{T}_{g, n}(\varepsilon)$ is compact follows from the Mumford compactness criterion for subsets of $\mathcal{M}_{g, n}$ [324].

Remark 5.21. The Mahler compactness criterion for subsets of the locally symmetric space $\mathrm{SL}(n, \mathbb{Z}) \backslash \mathrm{GL}^{+}(n, \mathbb{R}) / \mathrm{SO}(n)$, the space of lattices of $\mathbb{R}^{n}$, is a foundational result in the reduction theory of arithmetic groups. The Mumford compactness criterion was motivated by that, and this is another instance of fruitful interaction between two spaces discussed in this chapter.

The expected fact that $\mathcal{T}_{g, n}(\varepsilon)$ is a cofinite model of $\underline{E} \operatorname{Mod}_{g, n}$ was proved in [216].

Proposition 5.22. There exists a $\operatorname{Mod}_{g, n}$-equivariant deformation retraction of $\mathcal{T}_{g, n}$ to the thick part $\mathcal{T}_{g, n}(\varepsilon)$. In particular, for any finite subgroup $F \subset$ $\operatorname{Mod}_{g, n}$, the fixed point set $\mathcal{T}_{g, n}(\varepsilon)^{F}$ is nonempty and contractible, and hence $\mathcal{T}_{g, n}(\varepsilon)$ is a cofinite model of $\underline{\underline{E}} \operatorname{Mod}_{g, n}$.

It is clear that the thick part $\mathcal{T}_{g, n}(\varepsilon)$ is similar to the truncated subspace of the symmetric space $X$ mentioned in $\S 4.19$. Both spaces give cofinite models for universal spaces of proper actions.

\subsection{Cohomological properties of $\operatorname{Mod}_{g, n}$}

We now discuss some consequences of the existence of a cofinite $\underline{E} \operatorname{Mod}_{g, n}$-space in the previous subsection.

Proposition 5.23. $\operatorname{Mod}_{g, n}$ is of type WFL, which means that for any torsionfree subgroup $\Gamma^{\prime}$ of finite index there is a free resolution of $\mathbb{Z}$ over $\mathbb{Z} \Gamma^{\prime}$ of finite length, and $\operatorname{Mod}_{g, n}$ is also of type $F P_{\infty}$. In particular, in every degree $i$, $H_{i}\left(\operatorname{Mod}_{g, n}, \mathbb{Z}\right)$ and $H^{i}\left(\operatorname{Mod}_{g, n}, \mathbb{Z}\right)$ are finitely generated.

Determining the cohomology groups $H^{i}\left(\operatorname{Mod}_{g, n}, \mathbb{Z}\right)$ is important and complicated. The stable cohomology groups with rational coefficients $H^{i}\left(\operatorname{Mod}_{g, n}, \mathbb{Q}\right)$ can be computed (see [169] [188] [276] [275]). 
Another result is the following [169] [192].

Proposition 5.24. $\operatorname{Mod}_{g, n}$ is a virtual duality group and its dualizing module is equal to $H_{i}\left(\mathcal{C}\left(S_{g, n}\right), \mathbb{Z}\right)$, where $i$ is the only positive degree in which the homology of the curve complex $\mathcal{C}\left(S_{g, n}\right)$ is not equal to 0 . On the other hand, $\operatorname{Mod}_{g, n}$ is not a virtual Poincaré duality group.

The similarities with duality results for arithmetic subgroups in Proposition 4.67 are striking. The proof of these results are also similar, by using the fact that the boundary of $\mathcal{T}_{g, n}(\varepsilon)$ has the same homotopy type as the curve complex $\mathcal{C}\left(S_{g, n}\right)$ and the analogue of the Solomon-Tits theorem. This is one instance showing similar roles played by the Tits building $\Delta_{\mathbb{Q}}(\mathbf{G})$ and the curve complex $\mathcal{C}\left(S_{g, n}\right)$. For more results showing similarities between the Tits building $\Delta_{\mathbb{Q}}(\mathbf{G})$ and the curve complex $\mathcal{C}\left(S_{g, n}\right)$ and references, see [210].

\subsection{Pants decompositions and Bers constant}

An important technique in studying hyperbolic metrics on surfaces $S_{g, n}$ is to understand pants decompositions. The reason is that hyperbolic surfaces can be built up from the basic pieces, pairs of pants. For example, the FenchelNielsen coordinates can be defined for every pants decomposition.

Recall that for any hyperbolic surface $\Sigma_{g, n}$, a pants decomposition consists of a collection of simple closed geodesics $c_{1}, \cdots, c_{3 g-3+n}$ such that every connected component of the complement in $S_{g, n}$ is biholomorphic to the unit disk with two disjoint smaller disks removed, i.e., a pair of pants.

Pants decompositions are not unique. Since every essential simple closed curve in $\Sigma_{g, n}$, i.e., a curve not homotopic to a point or a loop around a puncture, contains a unique simple closed geodesic in its homotopy class, any collection of disjoint essential simple closed curves $\sigma_{1}, \cdots, \sigma_{3 g-3+n}$ in $\Sigma_{g, n}$ that are pairwise nonhomotopic induces a pants decomposition. Then any element $\phi \in \operatorname{Diff}^{+}\left(\Sigma_{g, n}\right), \phi\left(\sigma_{1}\right), \cdots, \phi\left(\sigma_{3 g-3+n}\right)$ also induce a pants decomposition, which is in general different from the previous one.

By the above argument, we can see that any collection of disjoint essential simple closed curves $\sigma_{1}, \cdots, \sigma_{3 g-3+n}$ in $S_{g, n}$ that are pairwise nonhomotopic induce a pants decomposition for every marked Riemann surface $\left(\Sigma_{g, n},[\varphi]\right)$ in $\mathcal{T}_{g, n}$.

A natural question is, for a given hyperbolic surface $\Sigma_{g, n}$, what kind of pants decompositions are optimal in some sense.

This is answered by the following basic result (see [90, Chap 5]).

Proposition 5.25. There exists a constant $\delta=\delta(g, n)$ such that every hyperbolic surface $\Sigma_{g, n}$ admits a pants decomposition such that the lengths of the geodesics in the pants decomposition are bounded from above by $\delta$. 
The constant $\delta(g, n)$ in the proposition is called a Bers constant. The minimal value it can take is not known. It is not clear how many pants decompositions satisfying the above conditions exist.

\subsection{Fundamental domains and rough fundamental domains}

Motivated by the reduction theory for arithmetic groups and its applications to understanding the structure of arithmetic groups and associated locally symmetric spaces, a natural and important problem for the action of $\operatorname{Mod}_{g, n}$ on $\mathcal{T}_{g, n}$ is to find fundamental domains and rough fundamental domains that reflect properties of $\operatorname{Mod}_{g, n}$ and $\mathcal{T}_{g, n}$. Besides their importance in understanding structures of mapping class groups, finding good fundamental domains is an interesting problem in itself.

The earlier discussion about the reduction theory of arithmetic subgroups indicates that it is not easy to construct fundamental domains. For many applications, rough fundamental domains with properties adapted to structures at infinity of Teichmüller spaces might be equally or even more useful than complicated fundamental domains.

In this subsection, we construct rough fundamental domains using the socalled Bers sets by using the pants decompositions that satisfy the conditions in Proposition 5.25. In the next section, we generalize Minkowski reduction to the action of $\operatorname{Mod}_{g}$ on $\mathcal{T}_{g}$ to obtain an intrinsically defined fundamental domain. This is closely related to a folklore open problem on constructing intrinsic $\operatorname{Mod}_{g}$-equivariant cell decompositions of $\mathcal{T}_{g}$ (see Problems 5.30 and 5.31 in the next subsection).

Before we define Bers sets, we summarize some earlier results on fundamental domains, rough fundamental domains for mapping class groups $\operatorname{Mod}_{g, n}$ and related $\operatorname{Mod}_{g, n}$-equivariant tiling of $\mathcal{T}_{g, n}$ :

(1) A fundamental domain for $\operatorname{Mod}_{g}$ in $\mathcal{T}_{g}$ was constructed and defined by finitely many equations involving lengths of (non-seperating) simple closed geodesics in [285] [286]. In some lower genus cases, defining equations are worked out in [287] [288] and [151] [152] more explicitly. The topology of the fundamental domain is not clear; for example, it is not known whether it is a cell.

(2) The Dirichlet fundamental domain for $\operatorname{Mod}_{g, n}$ with respect to the Teichmüller metric of $\mathcal{T}_{g, n}$ was studied in [297]. It is star-shaped with respect to the center. It is intrinsically defined in terms of the geometry of all Riemann surfaces in $\mathcal{T}_{g, n}$ once the center is chosen, but it is not defined in terms of the intrinsic geometry of each Riemann surface alone. It is not clear whether the closure in the augmented Teichmüller space $\hat{\mathcal{T}}_{g, n}$ is a cell. 
(3) A fundamental domain for $\operatorname{Mod}_{g}$ when $g=2$ was given in [229] explicitly in terms of nonlinear polynomials in some special coordinates of $\mathcal{T}_{g}$. The topology of the fundamental domain is not clear. There are also related results in [230].

(4) Rough (or approximate) fundamental domains for $\operatorname{Mod}_{g, n}$ were first introduced in [228] to solve a conjecture of Bers. Later in [90] and also implicitly in [3], different rough fundamental domains for $\operatorname{Mod}_{g, n}$ were introduced using Bers sets and Fenchel-Nielsen coordinates. This is analogous to the reduction theory for arithmetic groups acting on symmetric spaces of noncompact type (see [57] [61]).

(5) Equivariant cell decompositions of $\mathcal{T}_{g, n}$ for pairs $(g, n)$ with $n>0$ for small values $g$ and $n$ or for some subgroups of $\operatorname{Mod}_{g, n}$ were given in [375] [376]. Though equivariant cell decompositions of $\mathcal{T}_{g, n}$ are known in [169] [170] [65] [345] [346] [347] (see the next subsection for more detail), the point of the papers [375] [376] is to use systoles (minimal length of geodesics) to obtain such cell decompositions so that they might be generalized to the case $\mathcal{T}_{g}$.

(6) Generalizing the precise reduction theory of arithmetic groups acting on symmetric spaces of noncompact type [371], an equivariant tiling of $\mathcal{T}_{g}$ was given in [257]. (A tiling of a symmetric space means here an equivariant decomposition of the symmetric space. But each piece could have large stabilizers, and hence it is not an equivariant cell decomposition. See $\S 4.10$ for more detail.) To get an fundamental domain from this tiling, one needs to get a fundamental domain for each tile with respect to the stabilizer in $\operatorname{Mod}_{g}$ of the tile. The central tile is invariant under $\operatorname{Mod}_{g}$ with a compact quotient, and how to get such a fundamental domain of $\operatorname{Mod}_{g}$ for the central tile is not automatic or obvious. Getting a fundamental domain for other tiles depends on the central tile of Teichmüller spaces of smaller dimensions.

(7) In the case of genus 2, an equivariant cell decomposition of $\mathcal{T}_{g}$ was obtained in [367] using Weierstrass points.

Now we define the rough fundamental domains introduced in [228] [3] [90]. For every pants decomposition $\mathcal{P}=\left\{c_{1}, \cdots, c_{3 g-3+n}\right\}$ of $S_{g, n}$, there is a Fenchel-Nielsen coordinate system:

$$
\begin{gathered}
\mathcal{F N}_{\mathcal{P}}: \mathcal{T}_{g, n} \rightarrow \mathbb{R}_{+}^{3 g-3+n} \times \mathbb{R}^{3 g-3+n}, \\
\left(\Sigma_{g, n},[\varphi]\right) \mapsto\left(\ell_{1}, \cdots, \ell_{3 g-3+n}, \theta_{1}, \cdots, \theta_{3 g-3+n}\right),
\end{gathered}
$$

where $\ell_{i}$ is the length of the simple closed geodesic $\gamma_{i}$ in the homotopy class $\varphi^{-1}\left(c_{i}\right)$, and $\theta_{i}$ is the twisting angle along the geodesic $\gamma_{i}$. The twisting angles are not canonically defined and depend on an additional choice, for example, some extra combinatorial data. 
For each pants decomposition $\mathcal{P}$, for any positive constants $b_{1}, b_{2}$, define a Bers region

$$
\mathcal{B}_{\mathcal{P}, b_{1}, b_{2}}=\mathcal{F}_{\mathcal{P}}^{-1}\left(\left(0, b_{1}\right] \times \cdots \times\left(0, b_{1}\right] \times\left[-b_{2}, b_{2}\right] \times \cdots \times\left[-b_{2}, b_{2}\right]\right) .
$$

It is known that up to the action of $\operatorname{Mod}_{g, n}$, there are only finitely many equivalence classes of pants decompositions of $S_{g, n}$. Let $\mathcal{P}_{1}, \cdots, \mathcal{P}_{m}$ be representatives of these equivalence classes of pants decompositions.

Let $\delta=\delta(g, n)$ be the Bers constant in Proposition 5.25. For each $\mathcal{P}_{i}$, let $\mathcal{B}_{i}$ be the Bers region $\mathcal{B}_{\mathcal{P}_{i}, \delta, \pi}$.

Proposition 5.26. The union $\mathcal{B}=\mathcal{B}_{1} \cup \cdots \cup \mathcal{B}_{m}$ is a rough fundamental domain for the $\operatorname{Mod}_{g, n}$-action on $\mathcal{T}_{g, n}$. It satisfies both the local finiteness and the global finiteness conditions.

The fact that the $\operatorname{Mod}_{g, n}$-translates of $\mathcal{B}$ cover $\mathcal{T}_{g, n}$ follows from Proposition 5.25. For the proof that it is a rough fundamental domain satisfying the finiteness conditions, see $[90, \S 6.6]$.

Remark 5.27. As mentioned before, the curve complex $\mathcal{C}\left(S_{g, n}\right)$ is similar to the spherical Tits building $\Delta_{\mathbb{Q}}(\mathbf{G})$, and hence minimal $\mathbb{Q}$-parabolic subgroups of $\mathbf{G}$ correspond to pants decompositions $\mathcal{P}$ of $S_{g, n}$. The discussions above give a concrete example of such a comparison. The Fenchel-Nielsen coordinate system of $\mathcal{T}_{g, n}$ associated with $\mathcal{P}$ is similar to the horospherical decomposition of the symmetric space $X$ associated the minimal $\mathbb{Q}$-parabolic subgroup $\mathbf{P}$. Then the Bers subsets of $\mathcal{T}_{g, n}$ associated with $\mathcal{P}$ correspond to the Siegel subsets of $X$ associated with $\mathbf{P}$ in Definition 4.33. The horospherical decomposition of the symmetric space $X$ associated to non-minimal $\mathbb{Q}$-parabolic subgroups are important in the reduction theory of arithmetic groups and compactifications of the locally symmetric space $\Gamma \backslash X$. Similarly, there is also a generalization of the Fenchel-Nielsen coordinate system of $\mathcal{T}_{g, n}$ for any sub-collection of simple closed curves contained in any pants decomposition $\mathcal{P}$.

Proposition 5.26 is the analogue of the reduction theory for arithmetic groups in Proposition 4.34. As mentioned in the summary earlier in this subsection, an analogue of the reduction theory in [371], i.e., the $\Gamma$-equivariant tiling of $X$ recalled in $\S 4.10$, also holds for $\operatorname{Mod}_{g, n}[257]$.

\subsection{Generalized Minkowski reduction and fundamental domains}

After obtaining rough fundamental domains for the $\operatorname{Mod}_{g, n}$-action on $\mathcal{T}_{g, n}$ in the previous subsection, a natural problem is to construct a fundamental domain for $\operatorname{Mod}_{g, n}$. 
In the case of arithmetic groups $\Gamma$, there are two cases depending on whether the symmetric space $X$ is linear or not. If $X$ is linear, a fundamental domain for the $\Gamma$-action can be described and is given by the union of finitely many polyhedral cones in the associated symmetric cone. In the general case, the situation is more difficult and is not known.

For the action of $\operatorname{Mod}_{g, n}$ on $\mathcal{T}_{g, n}$, if $n>0$, a stronger result than constructing fundamental domains is known. Specifically, there is the following important result.

Proposition 5.28. Assume $n>0$. Then $\mathcal{T}_{g, n}$ admits an intrinsic $\operatorname{Mod}_{g, n^{-}}$ equivariant cell decomposition, and there are only finitely many $\operatorname{Mod}_{g, n}$-orbits of cells.

It is due to many people including Mumford, Thurston, Harer [169], Penner [345], Bowditch-Epstein [65]. This result is similar to the $\Gamma$-equivariant polyhedral cone decompositions of linear symmetric spaces.

An immediate corollary is

Corollary 5.29. The $\operatorname{Mod}_{g, n}$-action on $\mathcal{T}_{g, n}$ admits a fundamental domain consisting of finite cells in the equivariant cell decomposition in Proposition 5.28 .

Proposition 5.28 has several important applications.

(1) A proof of the Witten conjecture on the intersection theory of the moduli space $\mathcal{M}_{g, n}$ by Kontsevich [244]. (See also Chapter 5 of volume II of this Handbook [314] for a survey of Witten's conjecture and its various proofs.)

(2) Evaluation of the Euler characteristic of $\operatorname{Mod}_{g, n}$ by Harer-Zagier [171].

The method of proof of Proposition 5.28 depends crucially on the presence of punctures, i.e., $n>0$. Partially motivated by the above results, a longstanding folklore problem is the following.

Problem 5.30. Construct an intrinsic cell decomposition of $\mathcal{T}_{g}$ such that the following conditions are satisfied:

(1) It is equivariant with respect to $\operatorname{Mod}_{g}$ and there are only finitely many $\operatorname{Mod}_{g}$-equivalence classes of cells. (Naturally some cells are not closed since $\operatorname{Mod}_{g} \backslash \mathcal{T}_{g}$ is noncompact.)

(2) It descends to a finite cell decomposition of $\mathcal{M}_{g}$.

(3) The closure of each cell in the augmented Teichmüller space $\hat{\mathcal{T}}_{g}$ is a closed cell so that the cell decomposition of $\mathcal{M}_{g}$ extends to a finite cell decomposition of the Deligne-Mumford compactification $\overline{\mathcal{M}}_{g}{ }^{D M} \cong \operatorname{Mod}_{g} \backslash \hat{\mathcal{T}}_{g}$. 
It should be emphasized that the key point is that the cell decomposition should be intrinsic, i.e., depending on the intrinsic hyperbolic geometry or complex structure of the marked Riemann surfaces in $\mathcal{T}_{g}$. Otherwise, the existence of such an equivariant cell decomposition follows easily from general facts on the existence of triangulations of analytic spaces and the fact that $\mathcal{M}_{g}$ admits a compactification which is a projective variety (See [202]). The existence of an equivariant cell decompositions of $\mathcal{T}_{g}$ implies the existence of fundamental domains for the action of $\operatorname{Mod}_{g}$ on $\mathcal{T}_{g}$, which consist of a union of finitely many representatives of the cells. As one step towards solving Problem 5.30 , a weaker problem is to find an intrinsic fundamental domain for $\operatorname{Mod}_{g}$ which is the union of finitely many cells such that they have no overlap in the interior, and the closure of each cell in $\hat{\mathcal{T}}_{g}$ is also a cell. Then the $\Gamma$-translates of these cells give an equivariant decomposition of $\mathcal{T}_{g}$ with disjoint interior, and their closures in $\hat{\mathcal{T}}_{g}$ also give an equivariant decomposition of $\hat{\mathcal{T}}_{g}$ into cells. Therefore, a weaker version of Problem 5.30 is the following:

Problem 5.31. Construct a fundamental domain of the Mod ${ }_{g}$-action on $\mathcal{T}_{g}$ which consists of a finite union of cells such that these cells are defined intrinsically and their interiors are disjoint and their closures in the augmented Teichmüller space $\hat{\mathcal{T}}_{g}$ are also cells.

Remark 5.32. For a public statement of Problem 5.30 on equivariant intrinsic cell decompositions of $\mathcal{T}_{g}$ with an extension to the augmented space $\hat{\mathcal{T}}_{g}$, see Problems 1 and 2 by D. Sullivan of the CTQM problem list. In these problems, Sullivan proposed to use Weierstrass points of compact Riemann surfaces to replace the punctures to solve this problem. This list of open problem was created in 2006 at the opening symposium of Center for the Topology and Quantization of Moduli Spaces, University of Aarhus. It is posted as the website http://www.ctqm.au.dk/PL/ . It was also raised at a workshop on the moduli space of curves and is posted at

http://www.aimath.org/WWN/modspacecurves/open-problems/index.html

In this section, we discuss a generalization of Minkowski reduction for the action of $\operatorname{SL}(n, \mathbb{Z})$ on the space of positive definite quadratic forms to the action of $\operatorname{Mod}_{g}$ on $\mathcal{T}_{g}$, and hence give a solution to Problem 5.31. For more details, see [202].

The key concept is the notion of reduced ordered pants decomposition of a marked hyperbolic Riemann surface.

Let $\mathcal{P}=\left\{c_{1}, \cdots, c_{3 g-3}\right\}$ be an ordered collection of simple closed geodesics of a hyperbolic surface $\Sigma_{g}$ such that they form an ordered pants decomposition of $\Sigma_{g}$. It is called a reduced ordered pants decomposition of $\Sigma_{g}$ if the following conditions are satisfied:

(1) The geodesic $c_{1}$ has shortest length among all simple closed geodesics in $\Sigma_{g}$. 
(2) The geodesic $c_{2}$ has shortest length among all simple closed geodesics in $\Sigma_{g}$ that are disjoint from $c_{1}$.

(3) More generally, for any $i \geq 2, c_{i}$ has shortest length among all simple closed geodesics in $\Sigma_{g}$ that are disjoint from $c_{1}, \cdots, c_{i-1}$.

It is clear that such a reduced ordered pants decomposition always exists, but is not necessarily unique. It is not unique if and only if there are more than one simple closed geodesics of minimal length at some stages in the above definition. On the other hand, if $c_{1}$ is a unique simple closed geodesic of shortest length, i.e., a unique systole, and for every $i \geq 2, c_{i}$ is a unique simple closed geodesic of shortest length that is disjoint from $c_{1}, \cdots, c_{i-1}$, then $\left\{c_{1}, \cdots, c_{3 g-3}\right\}$ is a unique reduced ordered pants decomposition. It is clear that a generic hyperbolic surface $\Sigma_{g}$ has a unique reduced ordered pants decomposition.

For any ordered pants decomposition $\mathcal{P}=\left\{c_{1}, \cdots, c_{3 g-3}\right\}$ of $S_{g}$, define a domain $\tilde{\Omega}_{\mathcal{P}}$ of $\mathcal{T}_{g}$ as follows:

$\tilde{\Omega}_{\mathcal{P}}=\left\{\left(\Sigma_{g},[\varphi]\right) \in \mathcal{T}_{g} \mid\left[\varphi^{-1}(\mathcal{P})\right]\right.$ is a reduced ordered pants decomposition of $\left.\Sigma_{g}\right\}$,

where $\left[\varphi^{-1}(\mathcal{P})\right]$ represents the ordered pants decomposition of $\Sigma_{g}$ consisting of the unique geodesics in the homotopy classes $\left[\varphi^{-1}\left(c_{i}\right)\right], i=1, \cdots, 3 g-3$.

The domain $\tilde{\Omega}_{\mathcal{P}}$ is invariant under the stabilizer of $\mathcal{P}$ in $\operatorname{Mod}_{g}$, denoted by $\operatorname{Stab}_{\mathcal{P}}$. The reason is that if $\mathcal{P}$ is a reduced ordered pants decomposition for a marked Riemann surface $\left(\Sigma_{g},[\varphi]\right)$, then for any element $[\psi] \in \operatorname{Mod}_{g}, \psi(\mathcal{P})$ is also a reduced ordered pants decomposition of the new marked Riemann surface $[\psi] \cdot\left(\Sigma_{g},[\varphi]\right)$.

To construct a fundamental domain for the $\operatorname{Mod}_{g}$-action on $\mathcal{T}_{g}$, we need to find fundamental domains of $\operatorname{Stab}_{\mathcal{P}}$ in $\tilde{\Omega}_{\mathcal{P}}$. For this purpose, we need to identify $\operatorname{Stab}_{\mathcal{P}}$. It is clear that $\operatorname{Stab}_{\mathcal{P}}$ contains the subgroup generated by the Dehn twists along curves in $\mathcal{P}$. But it could also contain some half Dehn twists.

To explain this, we call a curve $c_{i} \in \mathcal{P}$ hyper-elliptic if $c_{i}$ separates off a one-holed torus, i.e., one connected component of $\Sigma_{g}-c_{i}$ is biholomorphic to a Riemann surface of genus 1 with a small disk removed.

Lemma 5.33. For every hyper-elliptic curve $c_{i} \in \mathcal{P}$, the half Dehn twist along $c_{i}$ is contained in $\operatorname{Mod}_{g}$ and also in the stabilizer $\operatorname{Stab}_{\mathcal{P}}$.

The idea of the proof is as follows. Each compact Riemann surface of genus 1 with one distinguished point admits an involution that fixes the distinguished point. Remove a small disk around this point that is stable under the involution. Then this involution corresponds to a half Dehn twist along the boundary circle. Let $\Sigma^{\prime}, \Sigma^{\prime \prime}$ be the two connected components of $\Sigma_{g}-c_{i}$. Suppose that $\Sigma^{\prime}$ is a one-holed torus. Then the involution on the pointed 
elliptic curve defines an involution on $\Sigma^{\prime}$. Extend it to $\Sigma^{\prime \prime}$ such that it is the identity map outside a small tubular neighborhood of $c_{i}$, and the extended map on $\Sigma_{g}$ is a half Dehn twist of $c_{i}$.

Given an ordered pants decomposition $\mathcal{P}$, let $\Gamma$ be the subgroup of $\operatorname{Mod}_{g}$ generated by the half Dehn twists on all hyper-elliptic curves in $\mathcal{P}$ and full Dehn twists on the other curves in $\mathcal{P}$. Then we have the following result.

Proposition 5.34. The stabilizer $\mathrm{Stab}_{\mathcal{P}}$ is equal to $\Gamma_{\mathcal{P}}$, and hence the domain $\tilde{\Omega}_{\mathcal{P}}$ is invariant under $\Gamma_{\mathcal{P}}$.

We note that in the definition of a reduced ordered pants decomposition, we have only imposed conditions on the lengths of the geodesics $c_{i}$ in $\left[\varphi^{-1}(\mathcal{P})\right]$. Since the Dehn twists along these geodesics do not change the fact that $\mathcal{P}$ is an ordered reduced pants decomposition and the lengths of these geodesics, it is natural to find conditions on the twisting parameters $\theta_{1}, \cdots, \theta_{3 g-3}$.

For each curve $c_{i} \in \mathcal{P}$, define $m_{i}=\frac{1}{2}$ if $c_{i}$ is hyper-elliptic, and $m_{i}=1$ otherwise. The choice of the value of $m_{i}$ is determined by the minimal Dehn twist along $c_{i}$ that is contained in $\operatorname{Stab}_{\mathcal{P}}$.

Define a subdomain $\Omega_{\mathcal{P}}$ of $\tilde{\Omega}_{\mathcal{P}}$ by

$\Omega_{\mathcal{P}}=\left\{\left(\ell_{1}, \theta_{1} ; \cdots ; \ell_{3 g-3}, \theta_{3 g-3}\right) \in \tilde{\Omega}_{\mathcal{P}} \mid \theta_{i} \in\left[0,2 \pi m_{i}\right], i=1, \cdots, 3 g-3\right\}$.

From the description of the stabilizer $\mathrm{Stab}_{\mathcal{P}}$, it is clear that the subdomain $\Omega_{\mathcal{P}}$ is a fundamental domain of the $\operatorname{Stab}_{\mathcal{P}}$-action on $\tilde{\Omega}_{\mathcal{P}}$.

For any pants decomposition $\mathcal{P}$, the twisting angles $\theta_{i}$ are not uniquely defined and depend on various choices. In [48], some particularly nice ones are chosen so that the length functions associated with simple closed geodesics are convex functions in the associated Fenchel-Nielsen coordinates.

A crucial property is the following result [202, Proposition 5.3].

Proposition 5.35. With respect to a suitable choice of Fenchel-Nielsen coordinates of $\mathcal{T}_{g}$ for each pants decomposition $\mathcal{P}$ in [48], $\Omega_{\mathcal{P}}$ is contractible.

The basic idea is to deform along the anti-stretch lines in the Thurston metric of $\mathcal{T}_{g}$ [404] so that in the deformation process, $\mathcal{P}$ is kept as a reduced ordered pants decomposition and the twisting coordinates remain invariant.

Let $\mathcal{P}_{1}, \cdots, \mathcal{P}_{n_{0}}$ be representatives of $\operatorname{Mod}_{g}$-equivalence classes of pants decompositions of $S_{g}$ as above. Let $\Omega_{\mathcal{P}_{1}}, \cdots, \Omega_{\mathcal{P}_{n_{0}}}$ be the domains associated with them as defined in Equation (4). Define

$$
\Omega=\Omega_{\mathcal{P}_{1}} \cup \cdots \cup \Omega_{\mathcal{P}_{n_{0}}} .
$$

Then one of the main results of [202] is the following, which gives a solution to Problem 5.31. 
Theorem 5.36. The domain $\Omega$ is an intrinsically defined fundamental domain for the Mod $_{g}$-action on $\mathcal{T}_{g}$ satisfying the following properties:

(1) It satisfies both the local finiteness and global finiteness conditions.

(2) Each domain $\Omega_{\mathcal{P}_{i}}$ in $\mathcal{T}_{g}$ is a cell and its closure $\overline{\Omega_{\mathcal{P}_{i}}}$ in $\hat{\mathcal{T}}_{g}$ is also a cell.

By the same argument, a fundamental domain for the action of $\operatorname{Mod}_{g, n}$ on $\mathcal{T}_{g, n}$ can be constructed and enjoys the same properties.

\subsection{Compactifications of moduli spaces and a conjecture of Bers}

Suppose that a discrete group $\Gamma$ acts properly on $X$ with a noncompact quotient $\Gamma \backslash X$. A natural and important problem is to understand relations between compactifications of $X$ and $\Gamma \backslash X$. If $\Gamma$ is infinite, the quotient of a compactification $\bar{X}$ of $X$ by $\Gamma$ is non-Hausdorff in general, since the $\Gamma$-action on the boundary is not proper since any infinite group cannot act properly on a compact space.

This problem has been discussed earlier in the setup of actions of arithmetic groups on symmetric spaces of noncompact type and compactifications of symmetric and locally symmetric spaces. Though this problem was known for a long time for compactification of the upper half plane and its quotients, it was Satake [374] who formulated it for general symmetric spaces and their arithmetic quotients.

In this section, we follow the method of Satake compactifications of locally symmetric spaces to construct the Deligne-Mumford compactification of $\mathcal{M}_{g, n}$ from the Bers compactification of $\mathcal{T}_{g, n}$. We believe that this might be the motivation for a conjecture of Bers [42, Conjecture IV, p. 599].

Near the end of this subsection, we also explain how to apply the same procedure to construct a new compactification of $\mathcal{M}_{g, n}$ whose boundary is equal to $\operatorname{Mod}_{g, n} \backslash \mathcal{C}\left(S_{g, n}\right)$, a finite simplicial complex.

Recall that for every fixed base point $\left(\Sigma_{g, n},[\varphi]\right)$ in $\mathcal{T}_{g, n}$, there is a Bers embedding

$$
i_{B}: \mathcal{T}_{g, n} \hookrightarrow \mathbb{C}^{3 g-3+n} \cong Q\left(\Sigma_{g, n}\right),
$$

where $Q\left(\Sigma_{g, n}\right)$ is the space of holomorphic quadratic differentials on $\Sigma_{g, n}$. It is a holomorphic embedding and the image is a bounded star-shaped domain. The closure $\overline{i_{B}\left(\mathcal{T}_{g, n}\right)}$ is the Bers compactification and it is denoted by ${\overline{\mathcal{T}_{g, n}}}^{B}$.

The geometry of the Bers boundary $\partial{\overline{\mathcal{T}_{g, n}}}^{B}$ is complicated. We can also define analytic arc components in $\partial{\overline{\mathcal{T}_{g}, n}}^{B}$ as for bounded symmetric domains. Though we cannot determine all the analytic arc components, it is known that the boundary $\partial{\overline{\mathcal{T}_{g, n}}}^{B}$ contains some natural complex submanifolds. 
In fact, it is known $[2, \S 5]$ (see also [299, Theorem 1.2, Corollay 1,3]) that every stable Riemann surface of Euler characteristic $2-2 g-n$ appears in the boundary $\partial{\overline{\mathcal{T}_{g, n}}}^{B}$. By [2, Corollary 1, p. 230], we have the following result.

Proposition 5.37. For every marked stable Riemann surface $\Sigma_{0}$ that is contained in the boundary $\partial{\overline{\mathcal{T}_{g, n}}}^{B}$, the Teichmüller space of $\Sigma_{0}$ is contained in the boundary $\partial{\overline{\mathcal{T}_{g, n}}}^{B}$ as well.

In [42, Conjecture IV, p. 599], Bers stated the following conjecture.

Conjecture 5.38. There exists a fundamental domain $\Omega$ for the $\operatorname{Mod}_{g, n^{-}}$ action on $\mathcal{T}_{g, n}$ such that the intersection of the closure of $\Omega$ in $\overline{\mathcal{T}}, n^{B}$ consists of cusps.

The notion of cusp is defined as follows. Let $\Sigma_{g, n}$ be the fixed base Riemann surface that defines the Bers emedding. Write it as a quotient $\Gamma_{\Sigma} \backslash \mathbf{H}^{2}$, where $\Gamma_{\Sigma}$ is a discrete subgroup of $P \mathrm{SL}(2, \mathbb{R})$. Then there is an embedding

$$
\mathcal{T}_{g, n} \rightarrow \operatorname{Hom}\left(\Gamma_{\Sigma}, \operatorname{PSL}(2, \mathbb{C})\right) / \text { conjugation } .
$$

The closure of this embedding can be identified with the Bers embedding $\overline{\mathcal{T}_{g, n}} B$ [299, p. 218]. Under this identification, and according to the definition in [42, p. 571] (see also [42, Theorem 10]), a boundary point in $\partial{\overline{\mathcal{T}_{g, n}}}^{B}$ given by a discrete faithful representation $\varphi: \Gamma_{\Sigma} \rightarrow \operatorname{PSL}(2, \mathbb{C})$ is called a cusp if a hyperbolic element in $\Gamma_{\Sigma}$ is mapped to a parabolic element.

A coarse fundamental domain for the $\operatorname{Mod}_{g, n}$-action on $\mathcal{T}_{g, n}$ was constructed in [228] and is was shown that the intersection of the closure of the rough fundamental domain with the boundary $\partial \overline{\mathcal{T}_{g, n}}$ consists of cusps.

An immediate corollary of [228] and the above discussion of fundamental is the following result.

Proposition 5.39. The fundamental domain $\Omega$ for the $M_{\text {od }}$-action on $\mathcal{T}_{g}$ in Theorem 5.36 satisfies the Bers conjecture. More generally, a similarly defined fundamental domain for the $\operatorname{Mod}_{g, n}$-action on $\mathcal{T}_{g, n}$ also satisfies the Bers conjecture.

Proof. By construction, the fundamental domain $\Omega$ in Theorem 5.36 is contained in the rough fundamental domains of [228], and the proposition follows immediately. Alternatively, we can see directly that for any unbounded sequence of marked hyperbolic surfaces in each domain $\Omega_{\mathcal{P}_{i}}$ in Theorem 5.36, some geodesics in the pants decomposition $\mathcal{P}_{i}$ are pinched, i.e., their lengths go to 0 . This implies that every boundary point in $\bar{\Omega} \cap \partial{\overline{\mathcal{T}_{g}}}^{B}$ is a cusp. 
Denote by $\Omega_{g, n}$ the fundamental domain for the action of $\operatorname{Mod}_{g, n}$ on $\mathcal{T}_{g, n}$. When $n=0$, it is reduced to $\Omega$ in Theorem 5.36.

It is clear that any regular $b$-group, i.e., a stable Riemann surface that appears in the boundary of $\partial{\overline{\mathcal{T}_{g}, n}}^{B}$, is a cusp. But the converse is not true in general. For example, assume $g \geq 3$. We can pinch one separating simple closed geodesic in $\Sigma_{g}$, and deform the connected component of genus at least 2 to a degenerate boundary point in the sense of [42] [1]. Then the corresponding boundary point of $\mathcal{T}_{g}$ is a cusp but not a $b$-regular group.

The following slightly stronger result holds and is important for the discussion of this section.

Proposition 5.40. The intersection of $\overline{\Omega_{g, n}} \cap \partial{\overline{\mathcal{T}_{g, n}}}^{B}$ consists of stable Riemann surfaces, and every marked stable Riemann surface belongs to a translate $\gamma \overline{\Omega_{g, n}}$ for some $\gamma \in \operatorname{Mod}_{g, n}$.

Proof. For simplicity, we discuss the case of $\mathcal{T}_{g}$. For a pants decomposition $\mathcal{P}_{i}$ in Theorem 5.36 and its associated domain $\Omega_{\mathcal{P}_{i}}$, if a sequence of marked Riemann surfaces $\left(\Sigma_{j},\left[\varphi_{j}\right]\right) \in \Omega_{\mathcal{P}_{i}}$ converges to a boundary point in $\overline{\Omega_{\mathcal{P}_{i}}} \cap \partial \overline{\mathcal{T}}_{g}{ }^{B}$, then by passing to a subsequence if necessary, we can assume that a subset of geodesics of $\left(\Sigma_{j},\left[\varphi_{j}\right]\right)$ contained in $\mathcal{P}_{i}$ is pinched, i.e., their lengths go to 0 , and for the other geodesics in $\mathcal{P}_{i}$, their lengths converge to positive numbers, and their Fenchel-Nielsen twisting parameters also converge. Such a sequence $\left(\Sigma_{j},\left[\varphi_{j}\right]\right)$ determines a marked stable Riemann surface $\left(\Sigma_{\infty},\left[\varphi_{\infty}\right]\right)$. By [299, Theorem 1.2, Corollary 1.3], the sequence $\left(\Sigma_{j},\left[\varphi_{j}\right]\right)$ also converges to $\left(\Sigma_{\infty},\left[\varphi_{\infty}\right]\right)$ in the Bers compactification $\overline{\mathcal{T}}_{g}{ }^{B}$. (Note that this is a crucial point. Of course, $\left(\Sigma_{j},\left[\varphi_{j}\right]\right)$ converges to $\left(\Sigma_{\infty},\left[\varphi_{\infty}\right]\right)$ in the augmented Teichmüller space $\widehat{\mathcal{T}}_{g}$ with respect to the three equivalent topologies in [1]. But we need the convergence with respect to the Bers compactification $\overline{\mathcal{T}}_{g}{ }^{B}$.) In the above proof, we have used the fact that the marked stable Riemann surface $\left(\Sigma_{\infty},\left[\varphi_{\infty}\right]\right)$ is contained in the Bers compactification $\overline{\mathcal{T}}_{g}^{B}$. This proves that the limit point of the sequence $\left(\Sigma_{j},\left[\varphi_{j}\right]\right) \in \Omega_{\mathcal{P}_{i}}$ in $\overline{\mathcal{T}}_{g}{ }^{B}$ is a stable Riemann surface, and the first statement is proved.

For the second statement, we note that for any marked stable Riemann surface $\left(\Sigma_{\infty},\left[\varphi_{\infty}\right]\right)$ of Euler characteristic $2-2 g-n$, by opening up the nodes, i.e., pairs of cusps, we obtain marked Riemann surfaces $\left(\Sigma_{j},\left[\varphi_{j}\right]\right)$ in $\mathcal{T}_{g}$. By passing to a suitable subsequence and under the action of some elements of the subgroup of $\operatorname{Mod}_{g}$ generated by the Dehn twists of the opened up geodesics, we can assume that $\left(\Sigma_{j},\left[\varphi_{j}\right]\right)$ is contained in $\gamma \Omega_{\mathcal{P}_{i}}$ for some pants decomposition $\mathcal{P}_{i}$ and $\gamma$ in the stabilizer $\operatorname{Stab}_{\mathcal{P}_{i}}$ of $\mathcal{P}_{i}$ in $\operatorname{Mod}_{g}$. Then the arguments in the previous paragraph show that $\left(\Sigma_{\infty},\left[\varphi_{\infty}\right]\right)$ is the limit of a subsequence of $\left(\Sigma_{j},\left[\varphi_{j}\right]\right)$ in the Bers compactification ${\overline{\mathcal{T}_{g}}}^{B}$, and hence is contained in the closure $\gamma \overline{\Omega_{P_{i}}}$. 
In constructing Satake compactifications of locally symmetric spaces $\Gamma \backslash X$, a boundary point of a Satake compactification of $X$ is called Siegel rational [61, p. 289] [374] if it meets the closure of a Siegel set of a $\mathbb{Q}$-parabolic subgroup.

Recall that we defined Bers sets $\mathcal{B}_{\mathcal{P}, b_{1}, b_{2}}$ in $\S 5.10$. For simplicity, denote it by $\mathcal{B}_{\mathcal{P}}$. Similarly we can introduce the following.

Definition 5.41. A boundary point in $\partial{\overline{\mathcal{T}_{g}, n}}^{B}$ is called rational if it is in the closure of a Bers set $\mathcal{B}_{\mathcal{P}}$ for some pants decomposition $\mathcal{P}$.

Then Proposition 5.40 says that the set of rational boundary points of $\overline{\mathcal{T}}_{g, n}{ }^{B}$ consists of exactly regular $b$-groups. The boundary Teichmüller spaces in Proposition 5.37 consists of rational points and hence can be called rational boundary components.

Now we can apply the method in defining Satake compactifications of locally symmetric spaces in [374] [61, §III. 3] to construct the Deligne-Mumford compactification of $\mathcal{M}_{g, n}$ and also to recover the topologies on the augmented Teichmüller space in [1].

By the above discussions, we have the following result.

Proposition 5.42. The augmented Teichmüller space $\hat{\mathcal{T}}_{g, n}$ mentioned in $\S 5.4$ is equal to the union of $\mathcal{T}_{g, n}$ with all rational boundary points of $\overline{\mathcal{T}}_{g, n} B$.

It is clear that $\operatorname{Mod}_{g, n}$ acts on $\hat{\mathcal{T}}_{g, n}$. For every Bers set $\mathcal{B}_{\mathcal{P}}$, the closure $\overline{\mathcal{B}_{\mathcal{P}}}$ in $\overline{\mathcal{T}}_{g, n}{ }^{B}$ is contained in $\hat{\mathcal{T}}_{g, n}$. Endow $\overline{\mathcal{B}_{\mathcal{P}}}$ with the subspace topology induced from the Bers compactification $\overline{\mathcal{T}}_{g, n} B$.

Proposition 5.43. There is a natural topology on $\hat{\mathcal{T}}_{g, n}$ that is induced from the topology of the Bers compactification $\overline{\mathcal{T}}_{g, n}{ }^{B}$ such that the action of $\operatorname{Mod}_{g, n}$ on $\hat{\mathcal{T}}_{g, n}$ satisfies the following properties:

(1) It induces the topology on $\mathcal{T}_{g, n}$ and the closure of every Bers set $\overline{\mathcal{B}_{\mathcal{P}}}$.

(2) The $\operatorname{Mod}_{g, n}$-action on $\hat{\mathcal{T}}_{g, n}$ is continuous.

(3) For every point $p \in \hat{\mathcal{T}}_{g, n}$, there exists a fundamental system of neighborhoods $\{U\}$ of $p$ such that for $\gamma$ in $\operatorname{Mod}_{g, n}$ that fixes $p, \gamma U=U$, and for the other $\gamma, \gamma U \cap U=\emptyset$.

(4) If $p, p^{\prime} \in \hat{\mathcal{T}}_{g, n}$ are not in one $\operatorname{Mod}_{g, n}$-orbit, then there exist neighborhoods $U$ of $p$ and $U^{\prime}$ of $p^{\prime}$ such that $\operatorname{Mod}_{g, n} U \cap U^{\prime}=\emptyset$.

Furthermore, any topology on $\hat{\mathcal{T}}_{g, n}$ satisfying the above conditions is equal to the natural one defined above. 
The basic idea is that for any boundary point $p$ contained in the closure $\overline{\mathcal{B}_{\mathcal{P}}}$ of a Bers set, take a neighborhood $V$ of $p$ in $\overline{\mathcal{B}_{\mathcal{P}}}$. Then the union of translates of $V$ by elements of the stabilizer of $p$ in $\operatorname{Mod}_{g, n}$ gives a neighborhood in the Satake topology of $\hat{\mathcal{T}}_{g, n}$. This can be seen clearly in the context of $\operatorname{SL}(2, \mathbb{Z})$ acting on $\mathbf{H}^{2}$ where horodisk neighborhoods of rational boundary points give the Satake topology.

The same proof of [374] works by noticing the fact that the induced action of $\operatorname{Mod}_{g, n}$ on each boundary Teichmüller space in ${\overline{\mathcal{T}_{g, n}}}^{B}$ is the action of corresponding mapping class groups and hence is proper.

It should be stressed that this Satake topology on $\hat{\mathcal{T}}_{g, n}$ is definitely different from (i.e., strictly finer than) the subspace topology on $\hat{\mathcal{T}}_{g, n}$ when it is considered as a subspace of $\overline{\mathcal{T}}, n^{B}$.

Remark 5.44. The proof of Proposition 5.40 shows that the topology of $\overline{\mathcal{B}_{\mathcal{P}}}$ is the same as the topologies induced from the three equivalent topologies on $\hat{\mathcal{T}}_{g, n}$ defined in [1]. Therefore, the Satake topology on the augmented Teichmüller space $\hat{\mathcal{T}}_{g, n}$ is equivalent to the three topologies in [1]. One important fact might be that the Satake topology here is defined using the topology of the Bers compactification $\overline{\mathcal{T}}_{g, n} B$. Therefore, we have constructed the augmented Teichmüller space $\hat{\mathcal{T}}_{g, n}$ (both the underlying space and the topology) purely in terms of the Bers compactification. This is in some sense similar to the fact that the Weil-Petersson completion of $\mathcal{T}_{g, n}$ gives an intrinsic construction of $\hat{\mathcal{T}}_{g, n}$ in Proposition 5.9.

An immediate corollary of Proposition 5.43 is the following.

Proposition 5.45. The quotient $\operatorname{Mod}_{g, n} \backslash \hat{\mathcal{T}}_{g, n}$ of $\hat{\mathcal{T}}_{g, n}$ with the Satake topology is a compact Hausdorff space, which is equal to the Deligne-Mumford compactification $\overline{\mathcal{M}}_{g, n} D M$.

Proof. The first statement follows from the properties of the Satake topology. The second statement follows from the construction of the DeligneMumford compactification $\overline{\mathcal{M}}_{g, n}{ }^{D M}$ that it is the moduli space of all stable Riemann surfaces of Euler characteristic $2-2 g-n$.

Remark 5.46. In his papers [40] [41] [42], Bers did not explain his motivations for making Conjecture IV of [42] (see also [40, p. 296] ), i.e., Conjecture 5.38 above. It seems that the above construction of the Deligne-Mumford compactification $\overline{\mathcal{M}}_{g, n}{ }^{D M}$ from the Bers compactification $\overline{\mathcal{T}}_{g, n} B$ following the method of compactifications of locally symmetric spaces should be one of the motivations. In some of his earlier works, Siegel had considered compactifications 
of fundamental domains of special arithmetic groups. Bers might have been motivated by some work of Siegel. The comments in [40, p. 296] might also justify the claim in this remark. On the other hand, it is important to note that compactifications of fundamental domains are related to, but different from, compactifications of locally symmetric spaces.

As discussed earlier in this chapter, besides the Bers compactification, the Teichmüller space $\mathcal{T}_{g, n}$ admits several compactifications. Among them, the Thurston compactification $\overline{\mathcal{T}}_{g, n}$ Th is probably the most interesting. A natural problem is to apply the above procedure to the Thurston compactification ${\overline{\mathcal{T}_{g, n}}}^{T h}$ and to construct the corresponding compactification of $\mathcal{M}_{g, n}$. It turns out to be a new compactification of $\mathcal{M}_{g, n}$ whose boundary is equal to $\operatorname{Mod}_{g, n} \backslash \mathcal{C}\left(S_{g, n}\right)$, a natural finite simplicial complex [205].

As in Definition 5.41, a boundary point of the Thurston compactification $\overline{\mathcal{T}}_{g, n}$ Th is called rational if it is contained in the closure of a Bers set $\mathcal{B}_{\mathcal{P}}$ of a pants decomposition $\mathcal{P}$.

It is known that the curve complex $\mathcal{C}\left(S_{g, n}\right)$ can be canonically embedded into the boundary of ${\overline{\mathcal{T}_{g, n}}}^{T h}$. Then the following result can be proved [198].

Proposition 5.47. For any Bers set $\mathcal{B}_{\mathcal{P}}$, the intersection of the closure of $\mathcal{B}_{\mathcal{P}}$ with the Thurston boundary $\partial \mathcal{B}_{\mathcal{P}}$ is equal to the simplex corresponding to the pants decomposition $\mathcal{P}$.

A corollary is the following result.

Corollary 5.48. The set of rational boundary points of $\overline{\mathcal{T}}_{g, n}$ Th is equal to the curve complex $\mathcal{C}\left(S_{g, n}\right)$.

Consequently, the partial compactification of $\mathcal{T}_{g, n}$ corresponding to the Thurston compactification $\overline{\mathcal{T}}_{g, n}$ Th is equal to $\mathcal{T}_{g, n} \cup \mathcal{C}\left(S_{g, n}\right)$, and the associated compactification of $\mathcal{M}_{g, n}$ is $\mathcal{M}_{g, n} \cup \operatorname{Mod}_{g, n} \backslash \mathcal{C}\left(S_{g, n}\right)$.

This compactification is similar to the Tits compactification of an arithmetic locally symmetric space $\Gamma \backslash X$ in $[215]$, whose boundary is $\Gamma \backslash \Delta_{\mathbb{Q}}(\mathbf{G})$, the quotient by $\Gamma$ of the Tits building $\Delta_{\mathbb{Q}}(\mathbf{G})$. Besides this formal similarity, the construction is also similar.

Remark 5.49. Naturally, we will also get a different compactification of $\mathcal{M}_{g, n}$ from the Teichmüller compactification of $\mathcal{T}_{g, n}$ by the above procedure. It would be interesting to identify this compactification. 


\subsection{Geometric analysis on moduli spaces}

The moduli space $\mathcal{M}_{g, n}$ has been extensively studied from the points of view of algebraic topology, complex geometry, algebraic geometry and mathematical physics etc.

In this section, we would like to raise several questions about $\mathcal{M}_{g, n}$ and emphasize the point of view of geometric analysis. The basic point is that $\mathcal{M}_{g, n}$ is also an important Riemannian orbifold and its geometry and analysis should be studied and better understood. We believe that this is an important direction to be explored.

The spectral theory of arithmetic locally symmetric spaces $\Gamma \backslash X$ has played a fundamental role in the theory of automorphis forms for $\Gamma$. A natural problem is to study the spectral theory of $\mathcal{M}_{g, n}$.

As mentioned before, $\mathcal{T}_{g, m}$ admits several $\operatorname{Mod}_{g, n}$-invariant Riemannian metrics, for example, the Weil-Petersson metric, the Bergman metric, the Ricci metric, the McMullen metric etc. They induce Riemannian metrics on $\mathcal{M}_{g, n}$. Though $\mathcal{M}_{g, n}$ is an orbifold, many concepts and techniques for Riemannian manifolds can be generalized to orbifolds and hence to $\mathcal{M}_{g, n}$. In particular, each Riemannian metric on $\mathcal{M}_{g, n}$ induces a Laplace operator.

Since the Weil-Petersson metric is incomplete, the first question is whether the Laplace operator with domain $C_{0}^{\infty}\left(\mathcal{M}_{g, n}\right)$ is essentially self-adjoint.

The answer seems to be positive. In a joint work in progress with $R$. Mazzeo, W. Müller, and A. Vasy, we expect to prove the following result.

Theorem 5.50. The Laplace operator of $\mathcal{M}_{g, n}$ acting on functions with respect to the Weil-Petersson metric is essentially self-adjoint and hence has a unique self-adjoint extension. Its spectrum is discrete and its counting function satisfies the Weyl law for the counting function of eigenvalues of compact Riemannian manifolds.

For other complete metrics such as the Bergman metric and the Ricci metric, it is known that the Laplace operator is essentially self-adjoint and has a unique self-adjoint extension. Using the asymptotic behaviors of these metrics near the infinity of $\mathcal{M}_{g, n}$, it can be shown that the spectrum of the Laplace operator is not discrete. On the other hand, it is not clear whether the non-discrete part of the spectrum is absolutely continuous, i.e., whether the spectrum measure is absolutely continuous. It is also desirable to understand structures of generalized eigenfunctions.

For Hermitian arithmetic locally symmetric spaces $\Gamma \backslash X$, an important result is the validity of the Zucker conjecture, which says that the $L^{2}$-cohomology group of $\Gamma \backslash X$ is canonically isomorphic to the intersection cohomology group of the Baily-Borel compactification of $\Gamma \backslash X$. The $L^{p}$-cohomology groups of $\Gamma \backslash X$ were also studied in [433]. See [217] in this volume for a more detailed discussion. 
A natural problem is to relate the $L^{2}$-cohomology group of $\mathcal{M}_{g, n}$ to some cohomology groups of compactifications of $\mathcal{M}_{g, n}$.

Probably the most natural and important compactification of $\mathcal{M}_{g, n}$ is the Deligne-Mumford compactification $\overline{\mathcal{M}}_{g, n} D M$. It is a compact orbifold and hence its intersection cohomology group is equal to the usual cohomology group.

Of course, the $L^{p}$-cohomolgy group of a Riemannian manifold (or an orbifold) depends only on the quasi-isometry class of the metric.

The following two results are proved in [217].

Proposition 5.51. For any $p$ with $4 / 3 \leq p<+\infty$, the $L^{p}$-cohomology group of $\mathcal{M}_{g, n}$ with respect to the Weil-Petersson metric is canonically isomorphic to the cohomology group of the Deligne-Mumford compactification ${\overline{\mathcal{M}_{g, n}}}^{D M}$. For $1 \leq p<4 / 3$, the $L^{p}$-cohomology group of $\mathcal{M}_{g, n}$ with respect to the WeilPetersson metric is canonically isomorphic to the cohomology group of $\mathcal{M}_{g, n}$.

The paper [372] proves only the case $\mathcal{M}_{g}$ and $p=2$, and the same proof works for the more general case $\mathcal{M}_{g, n}$ and $p=2$.

Proposition 5.52. With respect to any Riemannian metric that is quasiisometric to the Teichmüller metric, for any $1<p<\infty$, the $L^{p}$-cohomology group of $\mathcal{M}_{g, n}$ is isomorphic to the cohomology group of the Deligne-Mumford compactification $\overline{\mathcal{M}}_{g, n}$ DM .

In the case when the metric is the Bergman metric and $p=2$, this result was proved in [431, Theorem 4].

\section{Interactions between locally symmetric spaces and moduli spaces of Riemann surfaces}

The most basic example of a symmetric space is the upper half plane $\mathbf{H}^{2}=$ $\{x+i y \mid x \in \mathbb{R}, y>0\}$. It admits three important generalizations depending on different interpretations. First, $\mathbf{H}^{2}$ is the moduli space of marked elliptic curves (or Abelian varieties of dimension 1) and the quotient $\operatorname{SL}(2, \mathbb{Z}) \backslash \mathbf{H}^{2}$ is the moduli space of elliptic curves. Second, by writing $\mathbf{H}^{2}=\mathrm{SL}(2, \mathbb{R}) / \mathrm{SO}(2)$, we can identify it with the space of positive definite binary quadratic forms of determinant 1. Third, $\mathbf{H}^{2}$ is the Teichmüller space $\mathcal{T}_{g}$ when $g=1$.

The generalization based on the first interpretation is the Siegel upper half space $\mathfrak{h}_{g}=\{X+i Y \mid X, Y$ are real $g \times g$ matrices, $Y>0\}$. The symplectic group $\operatorname{Sp}(2 g, \mathbb{R})$ acts transitively and holomorphically on $\mathfrak{h}_{g}$, and the stabilizer 
of the point $i I_{g}$ is equal to $\mathrm{U}(g)$, and hence we have the identification:

$$
\mathfrak{h}_{g}=\operatorname{Sp}(2 g, \mathbb{R}) / \mathrm{U}(g) .
$$

It is a Hermitian symmetric space of noncompact type. The Siegel modular group $\operatorname{Sp}(2 g, \mathbb{Z})$ acts properly on $\mathfrak{h}_{g}$ and the quotient $\operatorname{Sp}(2 g, \mathbb{Z}) \backslash \mathfrak{h}_{g}$ is called the Siegel modular variety. It can be identified with the moduli space of principally polarized abelian varieties of dimension $g$ and usually denoted by $\mathcal{A}_{g}$. Clearly, when $g=1, \mathfrak{h}_{g}$ is equal to $\mathbf{H}^{2}$.

The generalization based on the second interpretation is the symmetric space $\mathrm{SL}(n, \mathbb{R}) / \mathrm{SO}(n)$. This space and its quotient $\mathrm{SL}(n, \mathbb{Z}) \backslash \mathrm{SL}(n, \mathbb{R}) / \mathrm{SO}(n)$ have been discussed before.

The generalization based on the third interpretation is the Teichmüller space $\mathcal{T}_{g}$ for $g \geq 2$, and $\operatorname{Mod}_{g}$ corresponds to $\operatorname{SL}(2, \mathbb{Z})$. The quotient space $\operatorname{Mod}_{g} \backslash \mathcal{T}_{g}$ is the moduli space $\mathcal{M}_{g}$. Of course, $\mathcal{T}_{g, n}$ and $\operatorname{Mod}_{g, n}$ are also natural generalizations.

\subsection{The Jacobian map and the Schottky problem}

It turns out that there is an important map between the two generalizations $\mathcal{M}_{g}$ and $\mathcal{A}_{g}$ of the space $\mathrm{SL}(2, \mathbb{Z}) \backslash \mathbf{H}^{2}$ in the previous paragraph, i.e., the Jacobian (or period) map

$$
J: \mathcal{M}_{g} \rightarrow \mathcal{A}_{g}
$$

Clearly, $\mathcal{A}_{g}=\operatorname{Sp}(2 g, \mathbb{Z}) \backslash \mathfrak{h}_{g}$ is an important locally symmetric space. In the previous sections, we were mainly interested in analogies between locally symmetric spaces and the moduli spaces $\mathcal{M}_{g, n}$. This Jacobian map establishes a direct connection between them.

We briefly recall its definition. Let $\Sigma_{g}$ be a compact Riemann surface of genus $g$, and let $A_{i}, B_{i}, i=1, \cdots, g$, be a symplectic basis of $H_{1}\left(\Sigma_{g}, \mathbb{Z}\right)$, i.e., a basis satisfying the conditions: for $i, j=1, \cdots, g$,

$$
A_{i} \cdot A_{j}=0, \quad B_{i} \cdot B_{j}=0, \quad A_{i} \cdot B_{j}=\delta_{i j} .
$$

Associated to this basis is a normalized basis $\left\{\omega_{1}, \ldots, \omega_{g}\right\}$ of the complex vector space $H^{0}\left(\Sigma_{g}, \Omega^{1}\right)$ of holomorphic 1-forms on $\Sigma_{g}$ satisfying the condition $\int_{A_{i}} \omega_{j}=\delta_{i j}$. The corresponding period matrix $\Pi=\left(\Pi_{i j}\right)$ of $\Sigma_{g}$ is the complex $g \times g$ matrix with entries defined by

$$
\Pi_{i j}=\int_{B_{i}} \omega_{j} .
$$

Riemann's bilinear relations $\left[154\right.$, p. 232] imply that $\Pi=\left(\Pi_{i j}\right)$ belongs to the Siegel upper half space $\mathfrak{h}_{g}$.

The choice of a different homology basis $A_{i}, B_{i}$ of $H_{1}\left(\Sigma_{g}, \mathbb{Z}\right)$ yields a new period $\Pi^{\prime}=\gamma \cdot \Pi$ for some $\gamma \in \operatorname{Sp}(2 g, \mathbb{Z})$. We thus have the well-defined period 
map

$$
J: \mathcal{M}_{g} \longrightarrow \mathcal{A}_{g}=\operatorname{Sp}(2 g, \mathbb{Z}) \backslash \mathfrak{h}_{g}
$$

which associates to (the isomorphism class of) a Riemann surface $\Sigma_{g}$ the $\operatorname{Sp}(2 g, \mathbb{Z})$-orbit through the period $\Pi$ above. This is the period map.

To explain the name of Jacobian map, we note that $L=\mathbb{Z}^{g} \oplus \Pi \mathbb{Z}^{g}$ is a lattice in $\mathbb{C}^{g}$ and the Jacobian variety $J\left(\Sigma_{g}\right)$ of the Riemann surface $\Sigma_{g}$ is the torus $L \backslash \mathbb{C}^{g}$, which turns out to be an abelian variety, i.e., it admits the structure of a projective variety. Moreover, the intersection pairing on homology $H_{1}\left(\Sigma_{g}, \mathbb{Z}\right)$ determines a Hermitian bilinear form on $\mathbb{C}^{g}$ with respect to which the torus $\mathbb{C}^{g} / L$ is principally polarized [154, p. 359]. Similarly, different choices of symplectic bases give rise to an isomorphism class of abelian varieties $\mathbb{Z}^{g} \oplus \Pi \mathbb{Z}^{g}$, i.e., its Jacobian variety $J\left(\Sigma_{g}\right)$. This gives the Jacobian map

$$
J: \mathcal{M}_{g} \longrightarrow \mathcal{A}_{g}
$$

Intrinsically, without using the period $\Pi$, the Jacobian variety $J\left(\Sigma_{g}\right)$ is equal to $H_{1}\left(\Sigma_{g}, \mathbb{Z}\right) \backslash\left(H^{0}\left(\Sigma_{g}, \Omega^{1}\right)\right)^{*}$, and the inclusion of $H_{1}\left(\Sigma_{g}, \mathbb{Z}\right)$ in the dual space $\left(H^{0}\left(\Sigma_{g}, \Omega^{1}\right)\right)^{*}$ is obtained by integrating 1 -forms along cycles in $H_{1}\left(\Sigma_{g}, \mathbb{Z}\right)$ [154, p. 307].

Remark 6.1. For another way to define the Jacobian map and an application of the Jacobian map to construct 2-forms on the moduli space $\mathcal{M}_{g}$, see [226].

By Torelli's Theorem (see [154, p. 359]), the Jacobian map $J$ is injective. When $g=1, \mathcal{M}_{g}=\mathcal{A}_{g}$, and $J$ is an isomorphism. For $g \geq 2, \operatorname{dim}_{\mathbb{C}} \mathcal{M}_{g}=3 g-3$ and $\operatorname{dim}_{\mathbb{C}} \mathfrak{h}_{g}=\frac{(g+1) g}{2}$. It can be shown that when $g=2,3$, the image $J\left(\mathcal{M}_{g}\right)$ is a Zariski dense subvariety of $\mathcal{A}_{g}$, and when $g \geq 4, J\left(\mathcal{M}_{g}\right)$ is a lower dimensional subvariety of $\mathcal{A}_{g}$.

The classical Schottky problem is to characterize the Jacobian locus $J\left(\mathcal{M}_{g}\right)$ inside the moduli space $\mathcal{A}_{g}$ of all principally polarized abelian varieties.

A lot of work has been devoted on this important problem since 1882 or earlier. Basically there are two approaches:

(1) the analytic one is to find polynomials that "cut out" the locus $J\left(\mathcal{M}_{g}\right)$ inside $\mathcal{A}_{g}$

(2) the geometric approach is to find geometric properties of principally polarized abelian varieties that are satisfied only by Jacobians.

It was finally proved in [388] [389] that a Jacobian variety is characterized by the condition that its Riemann theta function satisfies a nonlinear partial differential equation. See [325] for a history of the Schottky problem and [28] and [107] for more recent surveys of the status of the Schottky problem. 


\subsection{The coarse Schottky problem}

It is difficult to check whether a given abelian variety is a Jacobian variety using the characterization in [388] [389]. In order to construct explicit examples of abelian varieties that are not Jacobian varieties, Buser and Sarnak [91] studied the position of the Jacobian locus $J\left(\mathcal{M}_{g}\right)$ in $\mathcal{A}_{g}$ for large genera $g$ from the point of view of differential geometry. We note that $\mathfrak{h}_{g}$ has an invariant metric as a Riemannian symmetric space, and this metric induces a metric on $\mathcal{A}_{g}$. Buser and Sarnak consider a certain systolic function $m: \mathcal{A}_{g} \rightarrow \mathbb{R}$, i.e., the length of shortest geodesics of the abelian variety with a suitable normalized flat metric, which can be thought of as giving a "distance" to the boundary of $\mathcal{A}_{g}$. Then they prove that

$$
J\left(\mathcal{M}_{g}\right) \subset V_{g}:=\left\{x \in \mathcal{A}_{g} \mid m(x) \leq \frac{3}{\pi} \log (4 g+3)\right\} .
$$

Moreover, as $g \rightarrow+\infty, \operatorname{Vol}\left(V_{g}\right) / \operatorname{Vol}\left(\mathcal{A}_{g}\right)=O\left(g^{-\nu g}\right)$ for any $\nu<1$. The volumes are computed with respect to the volume form on $\mathcal{A}_{g}$ induced from the invariant metric. This means that for large genus $g$ the entire Jacobian locus lies in a "very small" neighborhood $V_{g}$ of the boundary of $\mathcal{A}_{g}$.

Motivated by this work of Buser and Sarnak, B. Farb proposed in [124, Problem 4.11] to study the Schottky problem from the point of view of large scale geometry, called the "Coarse Schottky Problem": How does $J\left(\mathcal{M}_{g}\right)$ look "from far away", or how "dense" is $J\left(\mathcal{M}_{g}\right)$ inside $\mathcal{A}_{g}$ in the sense of coarse geometry?

This question can be made precise by using the concept of an asymptotic cone (or tangent cone at infinity) introduced by Gromov. Recall that a sequence $\left(X_{n}, p_{n}, d_{n}\right)$ of unbounded, pointed metric spaces converges in the sense of Gromov-Hausdorff to a pointed metric space $(X, p, d)$ if for every $r>0$, the Hausdorff-distance between the balls $B_{r}\left(p_{n}\right)$ in $\left(X_{n}, d_{n}\right)$ and the ball $B_{r}(p)$ in $(X, d)$ goes to zero as $n \rightarrow \infty$.

Let $x_{0}$ be an (arbitrary) point of $\mathcal{A}_{g}$. The asymptotic cone of $\mathcal{A}_{g}$ endowed with the locally symmetric metric $d_{\mathcal{A}_{g}}$ is defined as the Gromov-Hausdorfflimit of rescaled pointed spaces:

$$
\mathrm{Cone}_{\infty}\left(\mathcal{A}_{g}\right):=\mathcal{G H}-\lim _{n \rightarrow \infty}\left(\mathcal{A}_{g}, x_{0}, \frac{1}{n} d_{\mathcal{A}_{g}}\right) .
$$

Note that $\mathrm{Cone}_{\infty}\left(\mathcal{A}_{g}\right)$ is independent of the choice of the base point $x_{0}$.

For some spaces asymptotic cones are easy to describe. For example, the asymptotic cone of the Euclidean space $\mathbb{R}^{n}$ is isometric to $\mathbb{R}^{n}$. Similarly, if $C$ is a cone in $\mathbb{R}^{n}$, then Cone ${ }_{\infty}(C)$ is isometric to $C$. The asymptotic cone of the Poincaré half place $\mathbf{H}^{2}$ is more complicated and turns out to be an $\mathbb{R}$-tree, i.e., a tree which branches everywhere. (Note that a usual simplicial tree branches at points that do not have any accumulation points.) For a hyperbolic Riemann surface $\Sigma_{g, n}$ with $n>0$, its $\operatorname{Cone}_{\infty}\left(\Sigma_{g, n}\right)$ is a "cone" over $n$ points, i.e., $n$ 
rays with a common origin. For Siegel's modular variety $\mathcal{A}_{g}$ with respect to the metric induced from the invariant metric of $\mathfrak{h}_{g}, \mathrm{Cone}_{\infty}\left(\mathcal{A}_{g}\right)$ is known to be isometric to a $g$-dimensional metric cone over a simplex, which is equal to $\operatorname{Sp}(2 g, \mathbb{Z}) \backslash \Delta_{\mathbb{Q}}(\operatorname{Sp}(2 g, \mathbb{C}))$ (see [215] for example).

Farb's question can now be stated as follows [124, Problem 4.11]:

Coarse Schottky problem: Describe, as a subset of a g-dimensional Euclidean cone, the subset of $\mathrm{Cone}_{\infty}\left(\mathcal{A}_{g}\right)$ determined by the Jacobian locus $J_{g}\left(\mathcal{M}_{g}\right)$ in $\mathcal{A}_{g}$.

One of the results of [212] gives a solution to the coarse Schottky problem. It asserts that the locus $J\left(\mathcal{M}_{g}\right)$ is coarsely dense.

Theorem 6.2. Let $\mathrm{Cone}_{\infty}\left(\mathcal{A}_{g}\right)$ be the asymptotic cone of Siegel's modular variety. Then the subset of $\mathrm{Cone}_{\infty}\left(\mathcal{A}_{g}\right)$ determined by the Jacobian locus $J\left(\mathcal{M}_{g}\right) \subset \mathcal{A}_{g}$ is equal to the entire Cone ${ }_{\infty}\left(\mathcal{A}_{g}\right)$. More precisely, $J\left(\mathcal{M}_{g}\right)$ is coarsely dense in $\mathcal{A}_{g}$, i.e., there exists a constant $\delta_{g}$ depending only on $g$ such that $\mathcal{A}_{g}$ is contained in a $\delta_{g}$-neighbourhood of $J\left(\mathcal{M}_{g}\right)$.

The basic idea of the proof is to degenerate a general compact Riemann surface $\Sigma_{g}$ to a stable Riemann surface such that each of its component is of genus 1 , and then apply the fact that the Jacobian map $J$ is an isomorphism when $g=1$.

It might be worthwhile to emphasize that in the theorem of [91] mentioned, the genus $g \rightarrow+\infty$, while $g$ is fixed here and hence there is no contradiction between these two seemingly opposite conclusions. The result of [91] implies that the constant $\delta_{g}$ in the above theorem goes to infinity as $g \rightarrow+\infty$. A natural problem is to estimate how fast $\delta_{g}$ goes to infinity.

Remark 6.3. The Jacobian map $J: \mathcal{M}_{g} \rightarrow \mathcal{A}_{g}$ has played an important role in the study of $\mathcal{M}_{g}$. For example, it was used in [242] to show that the moduli space of stable Riemann surfaces, the natural compactification of $\mathcal{M}_{g}$ which is equal to the later Deligne-Mumford compactification, is a projective variety.

For some related results on maps between locally symmetric spaces and $\mathcal{M}_{g}$, see [163].

\section{References}

[1] W. Abikoff, Degenerating families of Riemann surfaces, Ann. of Math. (2) 105 (1977), no. 1, 29-44.

[2] W. Abikoff, On boundaries of Teichmüller spaces and on Kleinian groups. III, Acta Math. 134 (1975), 211-237. 
[3] W. Abikoff, The real analytic theory of Teichmüller space, Lecture Notes in Mathematics, 820. Springer, 1980. vii+144 pp.

[4] S. Adyan, V. Durnev, Algorithmic problems for groups and semigroups, Russian Math. Surveys 55 (2000), no. 2, 207-296.

[5] P. Albuquerque, Patterson-Sullivan theory in higher rank symmetric spaces, Geom. Funct. Anal. 9 (1999) 1-28.

[6] R. Alperin, Locally compact groups acting on trees and property T, Monatsh. Math. 93 (1982), no. 4, 261-265.

[7] J. Amorós, M. Burger, K. Corlette, D. Kotschick, D. Toledo, Fundamental groups of compact Kähler manifolds, Mathematical Surveys and Monographs, 44. American Mathematical Society, Providence, RI, 1996. xii+140 pp.

[8] A. Ancona, Théorie du potentiel sur les graphes et les variétés, in École d'été de Probabilités de Saint-Flour XVIII-1988, pp. 1-112, Lecture Notes in Math., 1427, Springer, Berlin, 1990.

[9] J. Andersen, Mapping Class Groups do not have Kazhdan's Property (T), math.QA/0706.2184v1.

[10] B. Apanasov, Discrete groups in space and uniformization problems. Mathematics and its Applications (Soviet Series), 40. Kluwer Academic Publishers Group, Dordrecht, 1991. xviii+482 pp.

[11] B. Apanasov, Conformal geometry of discrete groups and manifolds. de Gruyter Expositions in Mathematics, 32. Walter de Gruyter \& Co., Berlin, 2000. xiv+523 pp.

[12] J. Arthur, The trace formula for noncompact quotient, Proceedings of the International Congress of Mathematicians, Vol. 1, 2 (Warsaw, 1983), 849-859, PWN, Warsaw, 1984.

[13] G. Arzhantseva, I. Lysenok, A lower bound on the growth of word hyperbolic groups, J. London Math. Soc. (2) 73 (2006), no. 1, 109-125

[14] A. Ash, Deformation retracts with lowest possible dimension of arithmetic quotients of self-adjoint homogeneous cones, Math. Ann. 225 (1977), no. 1, 6976.

[15] A. Ash, D. Mumford, M. Rapoport, Y. Tai, Smooth compactification of locally symmetric varieties, Math. Sci. Press, Brookline, Mass., 1975. iv+335 pp.

[16] J. Athreya, A. Bufetov, A. Eskin, M. Mirzakhani, Lattice Point Asymptotics and Volume Growth on Teichmüller space, arXiv:math/0610715.

[17] N. Avni, Arithmetic groups have rational representation growth, arXiv:0803.1331.

[18] P. Bahls, The isomorphism problem in Coxeter groups, Imperial College Press, London, 2005. xiv+176 pp.

[19] P. Bahls, Automorphisms of Coxeter groups, Trans. Amer. Math. Soc. 358 (2006), no. 4, 1781-1796. 
[20] W. Baily, A. Borel, Compactification of arithmetic quotients of bounded symmetric domains, Ann. of Math. (2) 841966 442-528.

[21] W. Ballmann, Nonpositively curved manifolds of higher rank, Ann. of Math. (2) 122 (1985), no. 3, 597-609.

[22] W. Ballmann, P. Eberlein, Fundamental groups of manifolds of nonpositive curvature, J. Differential Geom. 25 (1987), no. 1, 1-22.

[23] W. Ballmann, M. Gromov, V. Schroeder, Manifolds of nonpositive curvature, Progress in Mathematics, 61. Birkhäuser Boston, Inc., Boston, MA, 1985. vi+263 pp.

[24] A. Bartels, W. Lück, The Borel Conjecture for hyperbolic and CAT(0)-groups, arXiv:0901.0442.

[25] A. Bartels, W. Lück, H. Reich, The $K$-theoretic Farrell-Jones conjecture for hyperbolic groups, Invent. Math. 172 (2008), no. 1, 29-70.

[26] H. Bass, A.Lubotzky, Automorphisms of groups and of schemes of finite type, Israel J. Math. 44 (1983) 1-22.

[27] G. Baumslag, Automorphism groups of residually finite groups, J. London Math. Soc. 38 (1963) 117-118.

[28] A. Beauville, Le problème de Schottky et la conjecture de Novikov, Séminaire Bourbaki 1986/87, Astérisque 152-153 (1988), 101-112.

[29] A. Beardon, The geometry of discrete groups, Graduate Texts in Mathematics, 91. Springer-Verlag, New York, 1983. xii+337 pp.

[30] J. Behrstock, Asymptotic geometry of the mapping class group and Teichmüller space, Geom. Topol. 10 (2006), 1523-1578.

[31] J. Behrstock, C. Drutu, L. Mosher, Thick metric spaces, relative hyperbolicity, and quasi-isometric rigidity, Math. Ann. 344 (2009), no. 3, 543-595.

[32] J. Behrstock, B. Kleiner, Y. Minsky, L. Mosher, Geometry and rigidity of mapping class groups, arXiv:0801.2006.

[33] J. Behrstock, Y. Minsky, Dimension and rank for mapping class groups, Ann. of Math. (2) 167 (2008), no. 3, 1055-1077.

[34] M. Bekka, M. Cowling, P. de la Harpe, Some groups whose reduced $C^{*}$-algebra is simple, Inst. Hautes Études Sci. Publ. Math. No. 80 (1994) 117-134.

[35] B. Bekka, P. de la Harpe, A. Valette, Kazhdan's property (T), New Mathematical Monographs, 11. Cambridge University Press, Cambridge, 2008. xiv+472 pp.

[36] M. Belolipetsky, Counting maximal arithmetic subgroups. With an appendix by Jordan Ellenberg and Akshay Venkatesh. Duke Math. J. 140 (2007), no. 1, $1-33$.

[37] R. Benedetti, C. Petronio, Lectures on hyperbolic geometry. Universitext. Springer-Verlag, Berlin, 1992. xiv+330 pp.

[38] Y. Benoist, Propriétés asymptotiques des groupes linéaires Geom, Funct. Anal. 7 (1997), no. 1, 1-47. 
[39] Y. Benoist, Five lectures on lattices in semisimiple Lie groups, in - em Géométries à courbure négative ou nulle, groupes discrets et rigidités, 117-176, Sémin. Congr., 18, Soc. Math. France, Paris, 2009

[40] L. Bers, Uniformization, moduli, and Kleinian groups, Bull. London Math. Soc. 4 (1972), 257-300.

[41] L. Bers, Finite-dimensional Teichmüller spaces and generalizations, Bull. Amer. Math. Soc. (N.S.) 5 (1981), no. 2, 131-172.

[42] L. Bers, On boundaries of Teichmüller spaces and on Kleinian groups. I, Ann. of Math. (2) 91 (1970), 570-600.

[43] G. Besson, G. Courtois, S. Gallot, Entropies et rigidités des espaces localement symétriques de courbure strictement négative, Geom. Funct. Anal. 5 (1995), no. 5, 731-799.

[44] G. Besson, G. Courtois, S. Gallot, Volume et entropie minimale des espaces localement symétriques, Invent. Math. 103 (1991), no. 2, 417-445.

[45] M. Bestvina, The topology of $\operatorname{Out}\left(F_{n}\right)$, in Proceedings of the International Congress of Mathematicians, Vol. II (Beijing, 2002), 373-384, Higher Ed. Press, Beijing, 2002.

[46] M. Bestvina, N. Brady, Morse theory and finiteness properties of groups, Invent. Math. 129 (1997), no. 3, 445-470.

[47] M. Bestvina, K. Bromberg, K. Fujiwara, The asymptotic dimension of mapping class groups is finite, arXiv:1006.1939.

[48] M. Bestvina, K. Bromberg, K. Fujiwara, J. Souto, Shearing coordinates and convexity of length functions on Teichmueller space, arXiv:0902.0829.

[49] M. Bestvina, M. Feighn, The topology at infinity of $\operatorname{Out}\left(F_{n}\right)$, Invent. Math. 140 (2000), no. 3, 651-692.

[50] M. Bestvina, M. Feighn, Proper actions of lattices on contractible manifolds, Invent. Math. 150 (2002), no. 2, 237-256.

[51] M. Bestvina, M. Feighn, M. Handel, The Tits alternative for $\operatorname{Out}\left(F_{n}\right)$. I. Dynamics of exponentially-growing automorphisms, Ann. of Math. (2) 151 (2000), no. $2,517-623$.

[52] M. Bestvina, K. Fujiwara, A characterization of higher rank symmetric spaces via bounded cohomology, Geom. Funct. Anal. 19 (2009), no. 1, 11-40.

[53] M. Bestvina, G. Mess, The boundary of negatively curved groups, J. Amer. Math. Soc. 4 (1991), no. 3, 469-481.

[54] J. Birman, Braids, links, and mapping class groups, Annals of Mathematics Studies, No. 82. Princeton University Press, Princeton, 1974. ix +228 pp.

[55] J. Birman, A. Lubotzky, J. McCarthy, Abelian and solvable subgroups of the mapping class groups, Duke Math. J. 50 (1983), no. 4, 1107-1120.

[56] F. Bonahon, Low-dimensional geometry. From Euclidean surfaces to hyperbolic knots. Student Mathematical Library, 49. IAS/Park City Mathematical Subseries. American Mathematical Society, Providence, RI; Institute for Advanced 
Study (IAS), Princeton, NJ, 2009. xvi+384

[57] A. Borel, Introduction aux groupes arithmétiques, Hermann, Paris 1969, 125 pp.

[58] A. Borel, Compact Clifford-Klein forms of symmetric spaces, Topology 2 (1963) 111-122.

[59] A. Borel, Stable real cohomology of arithmetic groups, Ann. Sci. École Norm. Sup. (4) 7 (1974), 235-272.

[60] A. Borel, Some metric properties of arithmetic quotients of symmetric spaces and an extension theorem, J. Differential Geometry 6 (1972), 543-560.

[61] A. Borel, L. Ji, Compactifications of symmetric and locally symmetric spaces, Mathematics: Theory \& Applications. Birkhäuser Boston, Inc., Boston, MA, 2006. $\mathrm{xvi}+479 \mathrm{pp}$.

[62] A. Borel, J. P. Serre, Corners and arithmetic groups, Comment. Math. Helv. 48 (1973) 436-491.

[63] K. Bou-Rabee, T. Kaletha. Quantifying residual finiteness of arithmetic groups, arXiv:1008.3579.

[64] B. Bowditch, A course on geometric group theory, MSJ Memoirs, 16. Mathematical Society of Japan, Tokyo, 2006. x+104 pp.

[65] B. Bowditch, D. Epstein, Natural triangulations associated to a surface, Topology 27 (1988) 91-117.

[66] M. Bozejko, T. Januszkiewicz, R. Spatzier, Infinite Coxeter groups do not have Kazhdan's property, J. Operator Theory 19 (1988), no. 1, 63-67.

[67] T. Brendle, B. Farb, Every mapping class group is generated by 6 involutions, J. Algebra 278 (2004), no. 1, 187-198.

[68] M. Bridson, Non-positive curvature and complexity for finitely presented groups, International Congress of Mathematicians. Vol. II, 961-987, Eur. Math. Soc., Zürich, 2006.

[69] M. Bridson, Geometric and combinatorial group theory, in The Princeton companion to mathematics, edited by T. Gower, pp. 431 - 448, Princeton University Press, Princeton, NJ, 2008. xxii+1034 pp.

[70] M. Bridson, Semisimple actions of mapping class groups on CAT(0) spaces. in Geometry of Riemann surfaces, 1-14, London Math. Soc. Lecture Note Ser., 368, Cambridge Univ. Press, Cambridge, 2010

[71] M. Bridson, A. Haefliger, Metric spaces of non-positive curvature, Grundlehren der Mathematischen Wissenschaften, 319. Springer-Verlag, Berlin, 1999. xxii+643 pp.

[72] M. Bridson, P. de la Harpe, Mapping class groups and outer automorphism groups of free groups are $C^{*}$-simple, J. Funct. Anal. 212 (2004) 195-205.

[73] M. Bridson, K. Vogtmann, On the geometry of the automorphism group of a free group, Bull. London Math. Soc. 27 (1995), no. 6, 544-552. 
[74] M. Bridson, K. Vogtmann, Automorphisms of automorphism groups of free groups, J. Algebra 229 (2000), no. 2, 785-792.

[75] M. Bridson, K. Vogtmann, Automorphism groups of free groups, surface groups and free abelian groups, in Problems on mapping class groups and related topics, 301-316, Proc. Sympos. Pure Math., 74, Amer. Math. Soc., Providence, RI, 2006.

[76] J. Brock, The Weil-Petersson visual sphere, Geom. Dedicata 115 (2005), 1-18.

[77] J. Brock, B. Farb, Curvature and rank of Teichmüller space, Amer. J. Math. 128 (2006), no. 1, 1-22.

[78] H. Brown, R. Bülow, J. Neubüser, H. Wondratschek, H. Zassenhaus, Crystallographic groups of four-dimensional space. Wiley Monographs in Crystallography. Wiley-Interscience, New York-Chichester-Brisbane, 1978. xiv+443 pp.

[79] K. Brown, Cohomology of groups, Corrected reprint of the 1982 original. Graduate Texts in Mathematics, 87. Springer-Verlag, New York, 1994. x+306 pp.

[80] K. Brown, Finiteness properties of groups, J. Pure Appl. Algebra 44 (1987), no. 1-3, 45-75.

[81] K. Brown, R. Geoghegan, An infinite-dimensional torsion-free $\mathrm{FP}_{\infty}$ group, Invent. Math. 77 (1984), no. 2, 367-381.

[82] G. Brumfiel, The real spectrum compactification of Teichmüller space, in Geometry of group representations, Proc. AMS-IMS-SIAM Jt. Summer Res. Conf., Boulder/Colo. 1987, Contemp. Math. 74, 51-75 (1988).

[83] M. Bucher-Karlsson, Simplicial volume of locally symmetric spaces covered by $\mathrm{SL}_{3} \mathbb{R} / \mathrm{SO}(3)$, Geom. Dedicata 125 (2007), 203-224.

[84] M. Bucher-Karlsson, The simplicial volume of closed manifolds covered by $\mathbb{H}^{2} \times$ $\mathbb{H}^{2}$. (English summary) J. Topol. 1 (2008), no. 3, 584-602.

[85] I. Bumagina, The Hopf property for subgroups of hyperbolic groups, Geom. Dedicata 106 (2004), 211-230.

[86] U. Bunke, M. Olbrich, Scattering theory for geometrically finite groups, in Geometry, analysis and topology of discrete groups, pp. 40-136, Adv. Lect. Math. (ALM), 6, Int. Press, 2008.

[87] M. Burger, A. Iozzi, Alessandra, A. Wienhard, Surface group representations with maximal Toledo invariant, C. R. Math. Acad. Sci. Paris 336 (2003), no. 5, 387-390.

[88] M. Burger, N. Monod, Bounded cohomology of lattices in higher rank Lie groups, J. Eur. Math. Soc. 1 (1999), 199-235.

[89] K. Burns, R. Spatzier, Manifolds of nonpositive curvature and their buildings, Inst. Hautes Études Sci. Publ. Math. No. 65 (1987), 35-59.

[90] P. Buser, Geometry and spectra of compact Riemann surfaces, Progress in Mathematics, 106. Birkhäuser Boston, Inc., Boston, MA, 1992. xiv+454 pp.

[91] P. Buser, P. Sarnak, On the period matrix of a Riemann surface of large genus (with an appendix by J.H. Conway and N.J.A. Sloane), Invent. math. 117 (1994), $27-56$. 
[92] J. Cassels, An introduction to the geometry of numbers, Corrected reprint of the 1971 edition. Classics in Mathematics. Springer-Verlag, Berlin, 1997. viii+344 pp.

[93] J. Cassels, Rational quadratic forms, London Mathematical Society Monographs, 13. Academic Press, Inc. London-New York, 1978. xvi+413 pp.

[94] L. Charlap, Bieberbach groups and flat manifolds. Universitext. Springer-Verlag, New York, 1986. xiv+242 pp.

[95] R. Charney, J. Crisp, K. Vogtmann, Automorphisms of 2-dimensional rightangled Artin groups, Geom. Topol. 11 (2007), 2227-2264.

[96] I. Chatterji, K. Ruane, Some geometric groups with rapid decay, Geom. Funct. Anal. 15 (2005), no. 2, 311-339.

[97] H. Cohn, On the shape of the fundamental domain of the Hilbert modular group, Proc. Sympos. Pure Math., Vol. VIII, pp. 190-202, Amer. Math. Soc., 1965.

[98] H. Cohn, A numerical survey of the floors of various Hilbert fundamental domains, Math. Comp. 191965 594-605.

[99] M. Cowling, U. Haagerup, Completely bounded multipliers of the Fourier algebra of a simple Lie group of real rank one. Invent. Math. 96 (1989), 507-549.

[100] H. Coxeter, W. Moser, Generators and relations for discrete groups. Fourth edition. Ergebnisse der Mathematik und ihrer Grenzgebiete, 14. Springer-Verlag, Berlin-New York, 1980. ix+169 pp.

[101] M. Culler, J. Morgan, Group actions on R-trees, Proc. London Math. Soc. (3) 55 (1987), no. 3, 571-604.

[102] M. Culler and K. Vogtmann, Moduli of graphs and automorphisms of free groups, Invent. Math. 84 (1986), no. 1, 91-119.

[103] M. Culler and K. Vogtmann, A group-theoretic criterion for Property FA, Proc. Amer. Math. Soc. 124 (1996) 677-683.

[104] G. Daskalopoulos, R. Wentworth, Harmonic maps and Teichmüller theory. Handbook of Teichmüller theory. Vol. I, 33-109, IRMA Lect. Math. Theor. Phys., 11, Eur. Math. Soc., Zürich, 2007.

[105] M. Davis, The geometry and topology of Coxeter groups, London Mathematical Society Monographs Series, 32. Princeton University Press, Princeton, NJ, 2008. $\mathrm{xvi}+584 \mathrm{pp}$.

[106] P. de la Harpe, Topics in geometric group theory, Chicago Lectures in Mathematics. University of Chicago Press, Chicago, IL, 2000. vi+310 pp.

[107] O. Debarre, The Schottky problem: an update, Current topics in complex algebraic geometry, Math. Sci. Res. Inst. Publ. 28, Cambridge, 1995, 57-64.

[108] P.Deligne, G.Mostow, Monodromy of hypergeometric functions and non- lattice integral monodromy, Inst. Hautes Études Sci. Publ. Math. 63 (1986) 5-90.

[109] P. Deligne, D. Mumford, The irreducibility of the space of curves of given genus. Inst. Hautes Études Sci. Publ. Math. No. 361969 75-109. 
[110] T. Delzant, Sous-groupes distingués et quotients des groupes hyperboliques, Duke Math. J. 83 (1996), no. 3, 661-682.

[111] A. Dranishnikov, T. Januszkiewicz, Every Coxeter group acts amenably on a compact space, Topology Proc. 24 (1999), Spring, 135-141.

[112] C. Drutu, M. Kapovich, Lectures on Geometric Group Theory, preprint of a book, 2010, http://www.math.ucdavis.edu/ kapovich/EPR/ggt.pdf.

[113] C. Drutu, S. Mozes, M. Sapir, Divergence in lattices in semisimple Lie groups and graphs of groups, Trans. Amer. Math. Soc. 362 (2010), no. 5, 2451-2505.

[114] M. Duchin, K. Rafi, Divergence of geodesics in Teichmüller space and the mapping class group, Geom. Funct. Anal. 19 (2009), no. 3, 722-742.

[115] J. Duistermaat, V. Guillemin, The spectrum of positive elliptic operators and periodic bicharacteristics, Invent. Math. 29 (1975), no. 1, 39-79.

[116] J. Dymara, T. Januszkiewicz, Cohomology of buildings and their automorphism groups, Invent. Math. 150 (2002), no. 3, 579-627.

[117] P. Ehrlich, H. Im Hof, Dirichlet regions in manifolds without conjugate points, Comment. Math. Helv. 54 (1979), no. 4, 642-658.

[118] J. Elstrodt, F. Grunewald, J. Mennicke, Groups acting on hyperbolic space. Harmonic analysis and number theory. Springer Monographs in Mathematics. Springer-Verlag, Berlin, 1998. xvi+524 pp.

[119] M. Ershov, A. Jaikin-Zapirain, Property (T) for noncommutative universal lattices, Invent. Math. 179 (2010), no. 2, 303-347.

[120] A. Eskin, M. Mirzakhani, Counting closed geodesics in Moduli space, arXiv:0811.2362.

[121] E. Falbel, J. Parker, The geometry of the Eisenstein-Picard modular group, Duke Math. J. 131 (2006), no. 2, 249-289.

[122] B. Farb, The quasi-isometry classification of lattices in semisimple Lie groups, Math. Res. Lett. 4 (1997), no. 5, 705-717.

[123] B. Farb, Some problems on mapping class groups and moduli space, in Problems on mapping class groups and related topics, pp.11-55, Proc. Sympos. Pure Math., 74, Amer. Math. Soc., Providence, RI, 2006.

[124] B. Farb, Problems on mapping class groups and related topics, Proc. Sympos. Pure Math., 74, Amer. Math. Soc., Providence, RI, 2006, x+371 pp.

[125] B. Farb, M. Handel, Commensurations of $\operatorname{Out}\left(\mathrm{F}_{n}\right)$, Publ. Math. Inst. Hautes Études Sci. No. 105 (2007), 1-48.

[126] B. Farb, A. Lubotzky, Y. Minsky, Yair, Rank-1 phenomena for mapping class groups, Duke Math. J. 106 (2001), no. 3, 581-597.

[127] B. Farb, D.Magalit, A Primer on Mapping Class Groups, a preprint of a book, 2009.

[128] B. Farb, H. Masur, Teichmüller geometry of moduli space, I: Distance minimizing rays and the Deligne-Mumford compactification, rarXiv:0803.0141. 
[129] B. Farb, H. Masur, B. Farb and H. Masur - Teichmüller geometry of moduli space, II: $\mathcal{M}(S)$ seen from far away, in In the Tradition of Ahlfors-Bers, $V$, edited by M. Bonk, J. Gilman, H. Masur, Y. Minsky, and M. Wolf, pp. 71-79, Contemporary Mathematics, Volume 510, AMS, 2010.

[130] B. Farb, H. Masur, Geometry of the complex of curves. I. Hyperbolicity, Invent. Math. 138 (1999), no. 1, 103-149.

[131] B. Farb, S. Weinberger, The intrinsic asymmetry and inhomogeneity of Teichmüller space, arXiv:0804.4428, to appear To appear in Duke Math Jour.

[132] F. Farrell, Lectures on surgical methods in rigidity, Published for the Tata Institute of Fundamental Research, Bombay; by Springer-Verlag, Berlin, 1996. iv+98 pp.

[133] F. Farrell, L. Jones, Rigidity for aspherical manifolds with $\pi_{1} \subset \mathrm{GL}_{m}(R)$, Asian J. Math. 2 (1998), no. 2, 215-262.

[134] F. Farrell, P. Ontaneda, The Teichmüller space of pinched negatively curved metrics on a hyperbolic manifold is not contractible, Ann. of Math. (2) 170 (2009), no. 1, 45-65.

[135] W. Fenchel, J. Nielsen, Discontinuous groups of isometries in the hyperbolic plane. Edited and with a preface by Asmus L. Schmidt. Biography of the authors by Bent Fuglede. de Gruyter Studies in Mathematics, 29. Walter de Gruyter \& Co., Berlin, 2003. xxii+364 pp.

[136] S. Ferry, A. Ranicki, J. Rosenberg, Novikov conjectures, index theorems and rigidity, Vol. 1. London Mathematical Society Lecture Note Series, 226. Cambridge University Press, Cambridge, 1995. x+372 pp.

[137] E. Formanek, C. Procesi, The automorphism group of a free group is not linear, J. Algebra 149(2) (1992), 494-499.

[138] G. Francsics, P. Lax, Analysis of a Picard modular group, Proc. Natl. Acad. Sci. USA 103 (2006), no. 30, 11103-11105.

[139] G. Francsics, P. Lax, A semi-explicit fundamental domain for a Picard modular group in complex hyperbolic space, in Geometric analysis of PDE and several complex variables, 211-226, Contemp. Math., 368, Amer. Math. Soc., Providence, RI, 2005.

[140] R. Fricke, F. Klein, Vorlesungen über die Theorie der automorphen Funktionen. Band 1: Die gruppentheoretischen Grundlagen. Band II: Die funktionentheoretischen Ausführungen und die Andwendungen. Bibliotheca Mathematica Teubneriana, Bände 3, 4 Johnson Reprint Corp., New York; B. G. Teubner Verlagsgesellschaft, Stuttg art 1965 Band I: xiv+634 pp.; Band II: xiv+668 pp.

[141] K. Fujiwara, On non-bounded generation of discrete subgroups in rank-1 Lie group, in Geometry, spectral theory, groups, and dynamics, pp. 153-156, Contemp. Math., 387, Amer. Math. Soc., Providence, RI, 2005.

[142] K. Fujiwara, Quasi-homomorphisms on mapping class groups. Handbook of Teichmüller theory. Vol. II, 241-269, IRMA Lect. Math. Theor. Phys., 13, Eur. Math. Soc., Zürich, 2009. 
[143] S. Galatius, Stable homology of automorphism groups of free groups, arXiv:math/0610216.

[144] F. Gardiner, H. Masur, Extremal length geometry of Teichmüller space, Complex Variables Theory Appl. 16 (1991), no. 2-3, 209-237.

[145] H. Garland, M. Raghunathan, Fundamental domains for lattices in (R-)rank 1 semisimple Lie groups, Ann. of Math. (2) 921970 279-326.

[146] I. M. Gel'fand, M. Graev, I. Pyatetskii-Shapiro, Representation theory and automorphic functions, Academic Press, Inc., Boston, MA, 1990. xviii+426 pp.

[147] R. Geoghegan, Topological methods in group theory, Graduate Texts in Mathematics, 243. Springer, New York, 2008. xiv+473 pp.

[148] W. Goldman, Complex hyperbolic geometry, Oxford Mathematical Monographs. Oxford Science Publications. The Clarendon Press, Oxford University Press, New York, 1999. xx+316 pp.

[149] W. Goldman, Trace coordinates on Fricke spaces of some simple hyperbolic surfaces. Handbook of Teichmüller theory. Vol. II, 611-684, IRMA Lect. Math. Theor. Phys., 13, Eur. Math. Soc., Zürich, 2009.

[150] A. Gorodnik, H. Oh, Orbits of discrete subgroups on a symmetric space and the Furstenberg boundary, Duke Math. J. 139 (2007), no. 3, 483-525.

[151] D. Griffiths, The side-pairing elements of Maskit's fundamental domain for the modular group in genus two, Ann. Acad. Sci. Fenn. Math. 26 (2001), no. 1, $3-50$

[152] D. Griffiths, At most 27 length inequalities define Maskit's fundamental domain for the modular group in genus 2, in The Epstein birthday schrift, pp. 167-180, Geom. Topol. Monogr., 1, Geom. Topol. Publ., Coventry, 1998.

[153] D. Griffiths, Length inequalities for systems of geodesic loops on a surface of genus two. I, Bull. London Math. Soc. 28 (1996), no. 5, 505-508.

[154] P. Griffiths, J. Harris, Principles of algebraic geometry, John Wiley \& Sons, Inc., New York, 1994. xiv+813 pp.

[155] M. Gromov, Hyperbolic groups, in Essays in group theory, pp. 75-263, Math. Sci. Res. Inst. Publ., 8, Springer, 1987.

[156] M. Gromov, Asymptotic invariants of infinite groups, in Geometric group theory, Vol. 2 (Sussex, 1991), 1-295, London Math. Soc. Lecture Note Ser., 182, Cambridge Univ. Press, Cambridge, 1993.

[157] M. Gromov, I. Piatetski-Shapiro, Nonarithmetic groups in Lobachevsky spaces, Inst. Hautes Études Sci. Publ. Math. No. 66 (1988), 93-103.

[158] P. Gruber, C. Lekkerkerker, Geometry of numbers, Second edition. NorthHolland Mathematical Library, 37. North-Holland Publishing Co., Amsterdam, 1987. xvi+732 pp.

[159] F. Grunewald, D. Segal, The solubility of certain decision problems in arithmetic and algebra, Bull. Amer. Math. Soc. (N.S.) 1 (1979), no. 6, 915-918. 
[160] F. Grunewald, D. Segal, Some general algorithms. I. Arithmetic groups, Ann. of Math. (2) 112 (1980), no. 3, 531-583.

[161] F. Grunewald, D. Segal, Some general algorithms. II. Nilpotent groups, Ann. of Math. (2) 112 (1980), no. 3, 585-617.

[162] Y. Guivarc'h, L. Ji, J.C. Taylor, Compactifications of symmetric spaces, Progress in Mathematics, 156. Birkhäuser Boston, Inc., Boston, MA, 1998. xiv +284 pp.

[163] R. Hain, Locally symmetric families of curves and Jacobians, in Moduli of curves and abelian varieties, pp. 91-108, Aspects Math., E33, Vieweg, Braunschweig, 1999.

[164] R. Hain, E. Looijenga, Mapping class groups and moduli spaces of curves, in Algebraic geometry -Santa Cruz 1995, pp. 97-142, Proc. Sympos. Pure Math., 62, Part 2, Amer. Math. Soc., Providence, RI, 1997.

[165] U. Hamenstädt, Geometry of the mapping class groups. I. Boundary amenability, Invent. Math. 175 (2009), no. 3, 545-609.

[166] U. Hamenstädt, Geometry of the mapping class groups III: Quasi-isometric rigidity, arXiv:math.GT/0512429, revised version, January 2007.

[167] U. Hamenstädt, Geometric properties of the mapping class group, Problems on mapping class groups and related topics, pp. 215-232, Proc. Sympos. Pure Math., 74, Amer. Math. Soc., Providence, RI, 2006.

[168] G. Harder, A Gauss-Bonnet formula for discrete arithmetically defined groups, Ann. Sci. École Norm. Sup. (4) 4 (1971), 409-455.

[169] J. Harer, The cohomology of the moduli space of curves, in Theory of moduli, pp. 138-221, Lecture Notes in Math., 1337, Springer, 1988.

[170] J. Harer, The virtual cohomological dimension of the mapping class group of an orientable surface, Invent. Math. 84 (1986) 157-176.

[171] J. Harer, D. Zagier, The Euler characteristic of the moduli space of curves, Invent. Math. 85 (1986), no. 3, 457-485.

[172] W. Harvey, Boundary structure of the modular group, in Riemann surfaces and related topics, Proceedings of the 1978 Stony Brook Conference, pp. 245251, Ann. of Math. Stud., 97, Princeton Univ. Press, Princeton, N.J., 1981.

[173] W. Harvey, Geometric structure of surface mapping class groups, in Homological group theory (Proc. Sympos., Durham, 1977), pp. 255-269, London Math. Soc. Lecture Note Ser., 36, Cambridge Univ. Press, Cambridge-New York, 1979.

[174] W. Harvey, Teichmüller spaces, triangle groups and Grothendieck dessins. Handbook of Teichmüller theory. Vol. I, 249-292, IRMA Lect. Math. Theor. Phys., 11, Eur. Math. Soc., Zrich, 2007.

[175] A. Hatcher, K. Vogtmann, Homology stability for outer automorphism groups of free groups, Algebr. Geom. Topol. 4 (2004), 1253-1272.

[176] A. Hatcher, K. Vogtmann, The complex of free factors of a free group. Quart. J. Math. Oxford Ser. (2) 49 (1998), no. 196, 459-468. 
[177] T. Hattori, Asymptotic geometry of arithmetic quotients of symmetric spaces, Math. Z. 222 (1996), no. 2, 247-277.

[178] D. Hejhal, Eigenvalues of the Laplacian for Hecke triangle groups, Mem. Amer. Math. Soc. 97 (1992), no. 469, vi+165 pp.

[179] G. Hemion, On the classification of homeomorphisms of 2-manifolds and the classification of 3-manifolds, Acta Math. 142 (1979), no. 1-2, 123-155.

[180] F. Herrlich, G. Schmithüsen, On the boundary of Teichmüller disks in Teichmüller and in Schottky space, in Handbook of Teichmüller theory, Papadopoulos, A. (ed.), Volume I. Zürich: European Mathematical Society (EMS). IRMA Lectures in Mathematics and Theoretical Physics 11, 293-349 (2007).

[181] R.Holzapfel, Geometry and arithmetic around Euler partial differential equations, Mathematics and its Applications (East European Series), 11. D. Reidel Publishing Co., Dordrecht, 1986. 184 pp.

[182] R.Holzapfel, The ball and some Hilbert problems, Lectures in Mathematics ETH Zürich. Birkhäuser Verlag, Basel, 1995. viii+160 pp.

[183] P. Humbert, Théorie de la réduction des formes quadratiques définies positives dans un corps algébrique K fini, Comment. Math. Helv. 12, (1940) 263-306.

[184] J. Humphreys, Arithmetic groups. Lecture Notes in Mathematics, 789. Springer, Berlin, 1980. vii+158 pp.

[185] B. Hunt, The geometry of some special arithmetic quotients, Lecture Notes in Mathematics, 1637. Springer-Verlag, Berlin, 1996. xiv+332 pp.

[186] Y. Imayoshi, A construction of holomorphic families of Riemann surfaces over the punctured disk with given monodromy, in Handbook of Teichmüller theory, Vol. II, 93-130, IRMA Lect. Math. Theor. Phys., 13, Eur. Math. Soc., Zürich, 2009.

[187] Y. Imayoshi, M. Taniguchi, An introduction to Teichmüller spaces, SpringerVerlag, Tokyo, 1992. xiv+279 pp.

[188] N. Ivanov, Mapping class groups, in Handbook of geometric topology, pp. 523633, North-Holland, Amsterdam, 2002.

[189] N. Ivanov, Automorphism of complexes of curves and of Teichmüller spaces, Internat. Math. Res. Notices 14 (1997) 651-666.

[190] N. Ivanov, Subgroups of Teichmüller modular groups, Translations of Mathematical Monographs, 115. American Mathematical Society, Providence, RI, 1992. xii+127 pp.

[191] N. Ivanov, Fifteen problems about the mapping class groups, in Problems on mapping class groups and related topics, 71-80, Proc. Sympos. Pure Math., 74, Amer. Math. Soc., Providence, RI, 2006.

[192] N. Ivanov, L. Ji, Infinite topology of curve complexes and non-Poincaré duality of Teichmüller modular groups, Enseign. Math. (2) 54 (2008), no. 3-4, 381-395.

[193] N. Ivanov, J. McCarthy, On injective homomorphisms between Teichmüller modular groups. I, Invent. Math. 135 (1999), no. 2, 425-486. 
[194] B. Iversen, Hyperbolic geometry, London Mathematical Society Student Texts, 25. Cambridge University Press, Cambridge, 1992. xiv +298 pp.

[195] L. Ji, Mostow strong rigidity and nonisomorphism for outer automorphism groups of free groups and mapping class groups, in New developments in Lie theory and geometry, pp. 57-81, Contemp. Math., 491, Amer. Math. Soc., 2009.

[196] L. Ji, Steinberg representations and duality properties of arithmetic groups, mapping class groups, and outer automorphism groups of free groups, in Representation theory, pp. 71-98, Contemp. Math., 478, Amer. Math. Soc., 2009.

[197] L. Ji, Arithmetic groups and their generalizations. What, why, and how, AMS/IP Studies in Advanced Mathematics, 43. American Mathematical Society, Providence, RI; International Press, Cambridge, MA, 2008. xviii+259 pp.

[198] L. Ji, A summary of the work of Gregory Margulis, Pure Appl. Math. Q. 4 (2008), no. 1, Special Issue: In honor of Grigory Margulis. Part 2, 1-69.

[199] L. Ji, The integral Novikov conjectures for $S$-arithmetic groups. I, $K$-Theory 38 (2007), no. 1, 35-47.

[200] L. Ji, Asymptotic dimension and the integral $K$-theoretic Novikov conjecture for arithmetic groups, J. Differential Geom. 68 (2004), no. 3, 535-544.

[201] L. Ji, The integral Novikov conjectures for linear groups containing torsion elements, J. Topol. 1 (2008), no. 2, 306-316.

[202] L. Ji, Fundamental domains for mapping class groups and equivariant cell decomposition for Teichmüller spaces via Minkowski reduction, preprint, 2009.

[203] L. Ji, An equivariant spine of Teichmüller space of positive codimension, preprint.

[204] L. Ji, From symmetric spaces to buildings, curve complexes and outer spaces, Inovations in Incidence Geometry, 10 (2009) 33-80.

[205] L. Ji, Arithmetic groups, mapping class groups, related groups, and their associated spaces, in Proceedings of the Fourth International Congress of Mathematicians, AMS/IP Stud. Adv. Math., vol. 48, pp. 127-175, Amer. Math. Soc., Providence, RI, 2010.

[206] L. Ji, Integral Novikov conjectures and arithmetic groups containing torsion elements, Comm. Anal. Geom. 15 (2007), no. 3, 509-533.

[207] L. Ji, Lectures on locally symmetric spaces and arithmetic groups, in Lie groups and automorphic forms, pp. 87-146, AMS/IP Stud. Adv. Math., 37, Amer. Math. Soc., Providence, RI, 2006.

[208] L. Ji, Buildings and their applications in geometry and topology, Asian J. Math. 10 (2006), no. 1, 11-80.

[209] L. Ji, Simplicial volume of moduli spaces of Riemann surfaces, preprint 2010.

[210] L. Ji, Curve complexes versus Tits buildings: structures and applications, preprint, 2010.

[211] L. Ji, V. Kumar Murty, L. Saper, J. Scherk, The congruence subgroup kernel and the fundamental group of the reductive Borel-Serre compactification, 
preprint, 2010.

[212] L. Ji, E. Leuzinger, The asymptotic Schottky problem, Geom. Funct. Anal. 19 (2010) 1693-1712.

[213] L. Ji, Z. Lin, Finite generation properties of cohomology rings for infinite groups, preprint, 2010.

[214] L. Ji, K. Liu, X. Sun, S.T. Yau, in preparation.

[215] L. Ji, R. MacPherson, Geometry of compactifications of locally symmetric spaces, Ann. Inst. Fourier (Grenoble) 52 (2002), no. 2, 457-559.

[216] L. Ji, S. Wolpert, A cofinite universal space for proper actions for mappingclass groups, in In the Tradition of Ahlfors-Bers, V, edited by M. Bonk, J. Gilman, H. Masur, Y. Minsky, and M. Wolf, pp. 151-163, Contemporary Mathematics, Volume 510, AMS, 2010.

[217] L. Ji, S. Zucker, On $L^{p}$-cohomology and the geometry of metrics of moduli space of curves, in this volume, 2010.

[218] L. Ji, M. Zworski, Scattering matrices and scattering geodesics of locally symmetric spaces, Ann. Sci. École Norm. Sup. (4) 34 (2001), no. 3, 441-469.

[219] P. Jolissaint, Rapidly decreasing functions in reduced $C^{*}$-algebras of groups, Trans. Amer. Math. Soc. 317 (1990), no. 1, 167-196.

[220] I. Kapovich, M. Lustig, Intersection form, laminations and currents on free groups. Geom. Funct. Anal. 19 (2010), no. 5, 1426-1467.

[221] I. Kapovich, M. Lustig, Geometric intersection number and analogues of the curve complex for free groups. Geom. Topol. 13 (2009), no. 3, 1805-1833.

[222] I. Kapovich, N. Benakli, Boundaries of hyperbolic groups, in Combinatorial and geometric group theory, pp. 39-93, Contemp. Math., 296, Amer. Math. Soc., 2002.

[223] M. Kapovich, Hyperbolic manifolds and discrete groups. Reprint of the 2001 edition. Modern Birkhäuser Classics. Birkhäuser Boston, Inc., Boston, MA, 2009. xxviii+467 pp.

[224] M. Kapovich, J. Millson, On representation varieties of Artin groups, projective arrangements and the fundamental groups of smooth complex algebraic varieties. Inst. Hautes Études Sci. Publ. Math. No. 88 (1998), 5-95 (1999).

[225] S. Katok, Fuchsian groups. Chicago Lectures in Mathematics. University of Chicago Press, Chicago, IL, 1992. x+175 pp.

[226] N. Kawazumi, Canonical 2-forms on the moduli space of Riemann surfaces, in Handbook of Teichmüller theory, Vol. II, 217-237, IRMA Lect. Math. Theor. Phys., 13, Eur. Math. Soc., Zürich, 2009.

[227] D. Kazdan, G. Margulis, A proof of Selberg's hypothesis, Mat. Sb. (N.S.) 75 (117) 1968 163-168.

[228] L. Keen, A rough fundamental domain for Teichmüller spaces. Bull. Amer. Math. Soc. 83 (1977), no. 6, 1199-1226. 
[229] L. Keen, A modular group and Riemann surfaces of genus 2, Math. Z. 142 (1975), 205-219.

[230] L. Keen, On fundamental domains and the Teichmüller modular group, in Contributions to analysis, pp. 185-194. Academic Press, New York, 1974.

[231] L. Keen, N. Lakic, Hyperbolic geometry from a local viewpoint, London Mathematical Society Student Texts, 68. Cambridge University Press, Cambridge, 2007. $\mathrm{x}+271 \mathrm{pp}$.

[232] R. Kent, C. Leininger, Subgroups of mapping class groups from the geometrical viewpoint, In In the tradition of Ahlfors-Bers, IV, 119-141, Contemp. Math., 432, Amer. Math. Soc., Providence, RI, 2007.

[233] S. Kerckhoff, The Nielsen realization problem, Ann. of Math. (2) 117 (1983), no. 2, 235-265.

[234] S. Kerckhoff, The asymptotic geometry of Teichmüller space, . Topology 19 (1980), no. 1, 23-41.

[235] S. Kerckhoff, W. Thurston, Non-continuity of the action of the modular group at Bers' boundary of Teichmüller space, Invent. Math. 100 (1990), 25-48.

[236] Y. Kida, The mapping class group from the viewpoint of measure equivalence theory, Mem. Amer. Math. Soc. 196 (2008), no. 916, viii+190 pp.

[237] Y. Kida, Introduction to measurable rigidity of mapping class groups, in Handbook of Teichmüller theory, Vol. II, 297-367, IRMA Lect. Math. Theor. Phys., 13, Eur. Math. Soc., Zürich, 2009.

[238] Y. Kida, Measure equivalence rigidity of the mapping class group. Ann. of Math. (2) 171 (2010), no. 3, 1851-1901.

[239] D. Kleinbock, G. Margulis, Logarithm laws for flows on homogeneous spaces, Invent. Math. 138 (1999), no. 3, 451-494.

[240] G. Knieper, On the asymptotic geometry of nonpositively curved manifolds, Geom. Funct. Anal. 7 (1997), no. 4, 755-782.

[241] M. Knopp, On the cuspidal spectrum of the arithmetic Hecke groups, Math. Comp. 61 (1993), no. 203, 269-275.

[242] F. Knudsen, D. Mumford, The projectivity of the moduli space of stable curves. I. Preliminaries on "det" and "Div". Math. Scand. 39 (1976), no. 1, 19-55.

[243] M. Koecher, Beiträge zu einer Reduktionstheorie in Positivitätsbereichen. I, Math. Ann. 1411960 384-432.

[244] M. Kontsevich, Intersection theory on the moduli space of curves and the matrix Airy function, Comm. Math. Phys. 147 (1992), no. 1, 1-23.

[245] M. Kontsevich, Formal (non)commutative symplectic geometry. - em The Gel'fand Mathematical Seminars, 1990-1992, 173-187, Birkhäuser Boston, Boston, MA, 1993.

[246] D. Krammer, The conjugacy problem for Coxeter groups, Groups Geom. Dyn. 3 (2009) 71-171. 
[247] A. Krieg, Modular forms on half-spaces of quaternions, Lecture Notes in Mathematics, 1143. Springer-Verlag, Berlin, 1985. xiii+203 pp.

[248] S. Krstić, K. Vogtmann, Equivariant outer space and automorphisms of freeby-finite groups, Comment. Math. Helv. 68 (1993), no. 2, 216-262.

[249] S. Krushkal, B. Apanasov, N. Gusevskii, Kleinian groups and uniformization in examples and problems. Translations of Mathematical Monographs, 62. American Mathematical Society, Providence, RI, 1986. viii+198 pp.

[250] F. Labourie, Cross ratios, Anosov representations and the energy functional on Teichmüller space, Ann. Sci. Éc. Norm. Supér. (4) 41 (2008), no. 3, 437-469.

[251] J. Lafont, B. Schmidt, Simplicial volume of closed locally symmetric spaces of non-compact type, Acta Math. 197 (2006), no. 1, 129-143.

[252] R. Langlands, On the functional equations satisfied by Eisenstein series, Lecture Notes in Mathematics, Vol. 544. Springer-Verlag, Berlin-New York, 1976. $\mathrm{v}+337$ pp.

[253] M. Larsen, A. Lubotzky, Representation growth of linear groups. J. Eur. Math. Soc. (JEMS) 10 (2008), no. 2, 351-390.

[254] B. Leeb, A characterization of irreducible symmetric spaces and Euclidean buildings of higher rank by their asymptotic geometry. Bonner Mathematische Schriften, 326. Universität Bonn, Mathematisches Institut, Bonn, 2000. ii+42 pp.

[255] J. Lehner, Discontinuous groups and automorphic functions. Mathematical Surveys, No. VIII American Mathematical Society, Providence, R.I. 1964 xi+425 pp.

[256] E. Leuzinger, Tits geometry, arithmetic groups, and the proof of a conjecture of Siegel, J. Lie Theory 14 (2004) 317-338.

[257] E. Leuzinger, Reduction theory for mapping class groups and applications to moduli spaces, arXiv:0801.1589.

[258] G. Levitt, Automorphisms of hyperbolic groups and graphs of groups, Geom. Dedicata 114 (2005), 49-70.

[259] G. Link, Ergodicity of generalised Patterson-Sullivan measures in higher rank symmetric spaces, Math. Z. 254 (2006), no. 3, 611-625.

[260] K. Liu, X. Sun, S. T. Yau, Canonical metrics on the moduli space of Riemann surfaces. I, J. Differential Geom. 68 (2004), no. 3, 571-637.

[261] K. Liu, X. Sun, S. T. Yau, New results on the geometry of the moduli space of Riemann surfaces, Sci. China Ser. A 51 (2008), no. 4, 632-651.

[262] C. Löh, R. Sauer, Simplicial volume of Hilbert modular varieties, Comment. Math. Helv. 84 (2009), no. 3, 457-470.

[263] C. Löh, R. Sauer, Degree theorems and Lipschitz simplicial volume for nonpositively curved manifolds of finite volume, J. Topol. 2 (2009), no. 1, 193-225.

[264] E. Looijenga, $L^{2}$-cohomology of locally symmetric varieties, Compositio Math. 67 (1988), no. 1, 3-20. 
[265] A. Lubotzky, Subgroup growth and congruence subgroups, Invent. Math. 119 (1995), no. 2, 267-295.

[266] A. Lubotzky, Discrete groups, expanding graphs and invariant measures. With an appendix by Jonathan D. Rogawski. Reprint of the 1994 edition. Modern Birkhäuser Classics. Birkhäuser Verlag, Basel, 2010. iii+192 pp.

[267] A. Lubotzky, S. Mozes, M. Raghunathan, The word and Riemannian metrics on lattices of semisimple groups, Inst. Hautes Études Sci. Publ. Math. No. 91 (2000), 5-53 (2001).

[268] A. Lubotzky, D. Segal, Subgroup growth, Progress in Mathematics, 212. Birkhäuser Verlag, Basel, 2003. xxii+453 pp.

[269] W. Lück, Survey on geometric group theory, Münster J. Math. 1 (2008), 73-108.

[270] W. Lück, $L^{2}$-invariants: theory and applications to geometry and K-theory. A Series of Modern Surveys in Mathematics, 44. Springer-Verlag, Berlin, 2002. $\mathrm{xvi}+595 \mathrm{pp}$.

[271] F. Luo, Automorphisms of the complex of curves, Topology 39 (2000), no. 2, $283-298$.

[272] R. Lyndon, P.Schupp, Combinatorial group theory, Reprint of the 1977 edition. Classics in Mathematics. Springer-Verlag, 2001. xiv+339 pp.

[273] H. Maass, Siegel's modular forms and Dirichlet series, Dedicated to the last great representative of a passing epoch. Carl Ludwig Siegel on the occasion of his seventy-fifth birthday. Lecture Notes in Mathematics, Vol. 216. Springer-Verlag, Berlin-New York, 1971. v+328 pp.

[274] C. Maclachlan, A. Reid, The arithmetic of hyperbolic 3-manifolds. Graduate Texts in Mathematics, 219. Springer-Verlag, New York, 2003. xiv+463 pp.

[275] I. Madsen, Moduli spaces from a topological viewpoint, in International Congress of Mathematicians, Vol. I, 385-411, Eur. Math. Soc., Zürich, 2007.

[276] I. Madsen, M. Weiss, The stable moduli space of Riemann surfaces: Mumford's conjecture, Ann. of Math. (2) 165 (2007), no. 3, 843-941.

[277] A. Marden, Outer circles. An introduction to hyperbolic 3-manifolds. Cambridge University Press, Cambridge, 2007. xviii+427 pp.

[278] G. Margulis, Discrete subgroups of semisimple Lie groups, Ergebnisse der Mathematik und ihrer Grenzgebiete (3), 17. Springer-Verlag, Berlin, 1991. $\mathrm{x}+388$ pp.

[279] G. A. Margulis, On the decomposition of discrete subgroups into amalgams, Selected translations. Selecta Math. Soviet. 1 (1981), no. 2, 197-213.

[280] G. A. Margulis, G. A. Soifer, Maximal subgroups of infinite index in finitely generated linear groups. J. Algebra 69 (1981), no. 1, 1-23.

[281] R. MacPherson, M. McConnell, Explicit reduction theory for Siegel modular threefolds, Invent. Math. 111 (1993), no. 3, 575-625. 
[282] W. Magnus, Noneuclidean tesselations and their groups. Pure and Applied Mathematics, Vol. 61. Academic Press [A subsidiary of Harcourt Brace Jovanovich, Publishers], New York-London, 1974. xiv+207 pp.

[283] B. Maskit, Kleinian groups, Grundlehren der Mathematischen Wissenschaften, 287. Springer-Verlag, Berlin, 1988. xiv+326 pp.

[284] B. Maskit, On boundaries of Teiehmüller spaces and on Kleinian groups, II. Ann. of Math., 91 (1970), 607-639.

[285] B. Maskit, A picture of moduli space, Invent. Math. 126 (1996) 341-390.

[286] B. Maskit, On the fundamental domain for the Teichmúller modular group, J. Anal. Math. 75 (1998) 155-172.

[287] B. Maskit, Parameters for Fuchsian groups. II. Topological type $(1,1)$, Ann. Acad. Sci. Fenn. Ser. A I Math. 14 (1989), no. 2, 265-275.

[288] B. Maskit, Parameters for Fuchsian groups. I. Signature (0,4), in Holomorphic functions and moduli, Vol. II (Berkeley, CA, 1986), 251-265, Math. Sci. Res. Inst. Publ., 11, Springer, New York, 1988.

[289] H. Masur, Logarithmic law for geodesics in moduli space, in Mapping class groups and moduli spaces of Riemann surfaces, 229-245, Contemp. Math., 150, Amer. Math. Soc., Providence, RI, 1993.

[290] H. Masur, Geometry of Teichmüller space with the Teichmüller metric, to appear in Surveys in Differential Geometry, vol 14, 2010.

[291] H. Masur, Extension of the Weil-Petersson metric to the boundary of Teichmüller space, Duke Math. J. 43 (1976), no. 3, 623-635.

[292] H. Masur, M. Wolf, Teichmüller space is not Gromov hyperbolic, Ann. Acad. Sci. Fenn. Ser. A I Math. 20 (1995), no. 2, 259-267.

[293] H. Masur, M. Wolf, The Weil-Petersson isometry group, Geom. Dedicata 93 (2002), 177-190.

[294] K. Matsuzaki, M. Taniguchi, Hyperbolic manifolds and Kleinian groups. Oxford Mathematical Monographs. Oxford Science Publications. The Clarendon Press, Oxford University Press, New York, 1998. x+253 pp.

[295] J. McCarthy, "Tits-alternative" for subgroups of surface mapping class groups, Trans. Amer. Math. Soc. 291 (1985), no. 2, 583-612.

[296] J. McCarthy, A. Papadopoulos, The visual sphere of Teichmüller space and a theorem of Masur-Wolf, Ann. Acad. Sci. Fenn. Math. 24 (1999), no. 1, 147-154.

[297] J. McCarthy, A. Papadopoulos, Fundamental domains in Teichmüller space, Ann. Acad. Sci. Fenn. Math. 21 (1996), no. 1, 151-166.

[298] M. McConnell, Symmetric spaces and arithmetic groups, in Voronoi's impact on modern science, Book 1, Institute of Mathematics, National Academy of Sciences of Ukraine, pp. 155-185, Institute of Mathematics, Kyiv, 1998.

[299] C. McMullen, The moduli space of Riemann surfaces is Kähler hyperbolic, Ann. of Math. (2) 151 (2000), no. 1, 327-357. 
[300] C. McMullen, Cusps are dense, Ann. of Math. (2) 133 (1991), no. 1, 217-247.

[301] G. McShane, Simple geodesics and a series constant over Teichmüller space. Invent. Math. 132 (1998), no. 3, 607-632.

[302] D. Meintrup, T. Schick, A model for the universal space for proper actions of a hyperbolic group, New York J. Math. 8 (2002), 1-7.

[303] J. Milne, Lectures on Étale Cohomology, http://www.jmilne.org/math/CourseNotes/lec.html.

[304] I. Mineyev, G. Yu, The Baum-Connes conjecture for hyperbolic groups, Invent. Math. 149 (2002), no. 1, 97-122.

[305] H. Minkowski, Ausgewählte Arbeiten zur Zahlentheorie und zur Geometrie. [Selected papers on number theory and geometry] Mit D. Hilberts Gedächtnisrede auf H. Minkowski, Göttingen 1909. [With D. Hilbert's commemorative address in honor of H. Minkowski, Göttingen 1909] Edited and with a foreword and appendices by E. Krätzel and B. Weissbach. With English, French and Russian summaries. Teubner-Archiv zur Mathematik, 12. BSB B. G. Teubner Verlagsgesellschaft, Leipzig, 1989. 261 pp.

[306] Y. Minsky, Quasi-projections in Teichmüller space, J. Reine Angew. Math. 473 (1996), 121-136.

[307] Y. Minsky, Curve complexes, surfaces and 3-manifolds, International Congress of Mathematicians. Vol. II, 1001-1033, Eur. Math. Soc., Zürich, 2006.

[308] M. Mirzakhani, Growth of the number of simple closed geodesics on hyperbolic surfaces, Ann. of Math. (2) 168 (2008), no. 1, 97-125.

[309] M. Mirzakhani, Simple geodesics and Weil-Petersson volumes of moduli spaces of bordered Riemann surfaces. Invent. Math. 167 (2007), no. 1, 179-222.

[310] M. Mirzakhani, Simple geodesics and Weil-Petersson volumes of moduli spaces of bordered Riemann surfaces, Invent. Math. 167 (2007), no. 1, 179-222.

[311] M. Mirzakhani, Weil-Petersson volumes and intersection theory on the moduli space of curves, J. Amer. Math. Soc. 20 (2007), no. 1, 1-23.

[312] H. Miyachi, Teichmüller rays and the Gardiner-Masur boundary of Teichmüller space, Geom. Dedicata 137 (2008), 113-141.

[313] E. Moise, Geometric topology in dimensions 2 and 3, Graduate Texts in Mathematics, Vol. 47. Springer-Verlag, New York-Heidelberg, 1977. x+262 pp.

[314] G. Mondello, Riemann surfaces, ribbon graphs and combinatorial classes, in Handbook of Teichmüller theory, Vol. II, 151-215, IRMA Lect. Math. Theor. Phys., 13, Eur. Math. Soc., Zürich, 2009.

[315] N. Monod, An invitation to bounded cohomology, in International Congress of Mathematicians, Vol. II, 1183-1211, Eur. Math. Soc., Zürich, 2006.

[316] J. Morgan, P. Shalen, Valuations, trees, and degenerations of hyperbolic structures. I. Ann. of Math. (2) 120 (1984), no. 3, 401-476.

[317] S. Morita, Introduction to mapping class groups of surfaces and related groups, in Handbook of Teichmüller theory, Vol. I, 353-386, IRMA Lect. Math. Theor. 
Phys., 11, Eur. Math. Soc., Zürich, 2007.

[318] S. Morita, Structure of the mapping class groups of surfaces: a survey and a prospect, Proceedings of the Kirbyfest (Berkeley, CA, 1998), 349-406, Geom. Topol. Monogr., 2, Geom. Topol. Publ., Coventry, 1999.

[319] D. Morris, Bounded generation of SL $(n, A)$ (after D. Carter, G. Keller, and E. Paige), New York J. Math. 13 (2007) 383-421.

[320] L. Mosher, Mapping class groups are automatic, Ann. of Math. (2) 142 (1995), no. 2, 303-384.

[321] L. Mosher, Geometric survey of subgroups of mapping class groups, in Handbook of Teichmüller theory. Vol. I, 387-410, IRMA Lect. Math. Theor. Phys., 11, Eur. Math. Soc., Zürich, 2007.

[322] G. Mostow, Strong rigidity of locally symmetric spaces, Annals of Mathematics Studies, No. 78. Princeton University Press, 1973. v+195 pp.

[323] G. Moussong, S. Prassidis, Equivariant rigidity theorems, New York J. Math. 10 (2004) 151-167.

[324] D. Mumford, A remark on Mahler's compactness theorem, Proc. Amer. Math. Soc. 28 (1971) 289-294.

[325] D. Mumford, Curves and their Jacobians, The University of Michigan Press, Ann Arbor, Mich., 1975. vi+104 pp.

[326] V. K. Murty, Bounded and finite generation of arithmetic groups, in Number theory, pp. 249-261, CMS Conf. Proc., 15, Amer. Math. Soc., 1995.

[327] S. Nag, The complex analytic theory of Teichmüller spaces, Canadian Mathematical Society Series of Monographs and Advanced Texts. A Wiley-Interscience Publication. John Wiley \& Sons, Inc., New York, 1988. xiv+427 pp.

[328] M. Nathanson, On the fundamental domain of a discrete group, Proc. Amer. Math. Soc. 41 (1973), 629-630.

[329] A. Nevo, The spectral theory of amenable actions and invariants of discrete groups, Geom. Dedicata 100 (2003), 187-218.

[330] P. Nicholls, The ergodic theory of discrete groups, London Mathematical Society Lecture Note Series, 143. Cambridge University Press, Cambridge, 1989. $\mathrm{xii}+221 \mathrm{pp}$.

[331] V. Nikulin, I. Shafarevich, Geometries and groups. Universitext. Springer Series in Soviet Mathematics. Springer-Verlag, Berlin, 1987. viii+251 pp.

[332] K. Ohshika, Discrete groups, Translations of Mathematical Monographs, 207. Iwanami Series in Modern Mathematics. American Mathematical Society, Providence, RI, 2002. $\mathrm{x}+193 \mathrm{pp}$.

[333] N. Ozawa, Weak amenability of hyperbolic groups, Groups Geom. Dyn. 2 (2008), no. 2, 271-280.

[334] A. Papadopoulos, Handbook of Teichmüller theory, Vol. I. IRMA Lectures in Mathematics and Theoretical Physics, 11. European Mathematical Society (EMS), Zürich, 2007. viii+794 pp. 
[335] A. Papadopoulos, Handbook of Teichmüller theory, Vol. II. IRMA Lectures in Mathematics and Theoretical Physics, 13. European Mathematical Society (EMS), Zürich, 2009. x+874 pp.

[336] A. Papadopoulos, Introduction to Teichmüller theory, old and new, un Handbook of Teichmüller theory, Vol. I, 1-30, IRMA Lect. Math. Theor. Phys., 11, Eur. Math. Soc., Zürich, 2007.

[337] A. Papadopoulos, Metric spaces, convexity and nonpositive curvature, IRMA Lectures in Mathematics and Theoretical Physics, 6. European Mathematical Society (EMS), Zürich, 2005. xii+287 pp.

[338] A. Papadopoulos, G. Théret, On Teichmüller's metric and Thurston's asymmetric metric on Teichmüller space, in Handbook of Teichmüller theory, Vol. I, pp. 111-204, IRMA Lect. Math. Theor. Phys., 11, Eur. Math. Soc., Zürich, 2007.

[339] L. Paris, Braid groups and Artin groups, Handbook of Teichmüller theory, Vol. II, pp. 389-451, IRMA Lect. Math. Theor. Phys., 13, Eur. Math. Soc., Zürich, 2009 .

[340] L. Paris, D. Rolfsen, Geometric subgroups of mapping class groups, J. Reine Angew. Math. 521 (2000), 47-83.

[341] J. Parker, Complex hyperbolic lattices, in Discrete groups and geometric structures, 1-42, Contemp. Math., 501, Amer. Math. Soc., Providence, RI, 2009.

[342] J. Parker, Cone metrics on the sphere and Livné's lattices, Acta Math. 196 (2006), no. 1, 1-64.

[343] S. J. Patterson, The limit set of a fuchsian group, Acta mathematica 136 (1976) 241-273.

[344] F. Paulin, Sur les automorphismes de groupes libres et de groupes de surface, Version préliminaire, Séminaire Bourbaki 1023 (Juin 2010).

[345] R. Penner, The decorated Teichmüller space of puctured surfaces, Communications in Mathematical Physics 113 (1987) 299-339.

[346] R. Penner, Cell decomposition and compactification of Riemann's moduli space in decorated Teichmüller theory, Woods Hole mathematics, pp. 263-301, Ser. Knots Everything, 34, World Sci. Publ., Hackensack, NJ, 2004.

[347] R. Penner, Perturbative series and the moduli space of Riemann surfaces, Journal of Differential Geometry 27 (1988) 35-53.

[348] V. Platonov, A. Rapinchuk, Algebraic groups and number theory, Pure and Applied Mathematics, 139. Academic Press, Inc., Boston, MA, 1994. xii+614 pp.

[349] V. Platonov, A. Rapinchuk, Abstract properties of $S$-arithmetic groups and the congruence problem, Russian Acad. Sci. Izv. Math. 40 (1993), no. 3, 455-476

[350] S. Popa, Deformation and rigidity for group actions and von Neumann algebras. International Congress of Mathematicians. Vol. I, 445-477, Eur. Math. Soc., Zürich, 2007. 
[351] G. Prasad, Discrete subgroups isomorphic to lattices in semisimple Lie groups, Amer. J. Math. 98 (1976), no. 1, 241-261.

[352] G. Prasad, Volumes of $S$-arithmetic quotients of semi-simple groups, Inst. Hautes Études Sci. Publ. Math. No. 69 (1989), 91-117.

[353] G. Prasad, A. Rapinchuk, Zariski-dense subgroups and transcendental number theory, Math. Res. Lett. 12 (2005), no. 2-3, 239-249.

[354] G. Prasad, A. Rapinchuk, Developments on the congruence subgroup problem after the work of Bass, Milnor and Serre, preprint, preprint, 2009.

[355] S. Prassidis, B. Spieler, Rigidity of Coxeter groups, Trans. Amer. Math. Soc. 352 (2000), no. 6, 2619-2642.

[356] M. Puschnigg, The Kadison-Kaplansky conjecture for word-hyperbolic groups, Invent. Math. 149 (2002), no. 1, 153-194.

[357] D. Quillen, The spectrum of an equivariant cohomology ring. II. - em Ann. of Math. (2) 94 (1971), 573-602.

[358] J. F. Quint, Mesures de Patterson-Sullivan en rang supérieur, Geom. Funct. Anal. 12 (2002), no. 4, 776-809.

[359] K. Rafi, A combinatorial model for the Teichmüller metric, Geom. Funct. Anal. 17 (2007), no. 3, 936-959.

[360] M. Raghunathan, Discrete subgroups of Lie groups, Springer-Verlag, New YorkHeidelberg, 1972. ix +227 pp.

[361] M. Raghunathan, The congruence subgroup problem, Proceedings of the Hyderabad Conference on Algebraic Groups (Hyderabad, 1989), pp. 465-494, Manoj Prakashan, Madras, 1991.

[362] M. Raghunathan, The congruence subgroup problem, Proc. Indian Acad. Sci. Math. Sci. 114 (2004), no. 4, 299-308.

[363] J. Ratcliffe, Foundations of hyperbolic manifolds. Second edition. Graduate Texts in Mathematics, 149. Springer, New York, 2006. xii+779 pp.

[364] D. B. McReynolds, The congruence subgroup problem for braid groups: Thurston's proof, arXiv:0901.4663.

[365] H. E. Rauch, A transcendental view of the space of algebraic Riemann surfaces, Bull. Amer. Math. Soc. 71 (1965) 1-39.

[366] D. Robinson, A course in the theory of groups, Second edition. Graduate Texts in Mathematics, 80. Springer-Verlag, New York, 1996. xviii+499 pp.

[367] A. Rodado, Weierstrass points and canonical cell decompositions of the moduli and Teichmüller Spaces of Riemann surfaces of genus two, PhD thesis, Mathematics and Statistics, The university of Melbourne, 2007.

[368] J. Roe, Hyperbolic groups have finite asymptotic dimension, Proc. Amer. Math. Soc. 133 (2005), no. 9, 2489-2490.

[369] S. Ryshkov, The theory of hermite-Minkowski reduction of positive definite quadratic forms, Journal of Mathematical Sciences, 6 (1976) 651-671. 
[370] S. Ryshkov, Geometry of positive quadratic forms, Proceedings of the International Congress of Mathematicians (Vancouver, B.C., 1974), Vol. 1, pp. 501-506. Canad. Math. Congress, Montreal, Que., 1975.

[371] L. Saper, Tilings and finite energy retractions of locally symmetric spaces, Comment. Math. Helv. 72 (1997), no. 2, 167-202.

[372] L. Saper, $L^{2}$-cohomology of the Weil-Petersson metric, in Mapping class groups and moduli spaces of Riemann surfaces, pp. 345-360, Contemp. Math., 150, Amer. Math. Soc., Providence, RI, 1993.

[373] L. Saper, M. Stern, $L_{2}$-cohomology of arithmetic varieties, Ann. of Math. (2) 132 (1990), no. 1, 1-69.

[374] I. Satake, On compactifications of the quotient spaces for arithmetically defined discontinuous groups, Ann. of Math. (2) 721960 555-580.

[375] P. Schmutz Schaller, A systolic geometric cell decomposition for the space of once-holed Riemann surfaces of genus 2, Topology 40 (2001), no. 5, 1017-1049.

[376] P. Schmutz Schaller, A cell decomposition of Teichmüller space based on geodesic length functions, Geom. Funct. Anal. 11 (2001), no. 1, 142-174.

[377] P. Schmutz Schaller, Systoles and topological Morse functions for Riemann surfaces, J. Differential Geom. 52 (1999), no. 3, 407-452.

[378] P. Schmutz Schaller, Geometry of Riemann surfaces based on closed geodesics, Bull. Amer. Math. Soc. (N.S.) 35 (1998), no. 3, 193-214.

[379] J. Schwermer, Geometric cycles, arithmetic groups and their cohomology, Bull. Amer. Math. Soc. 47 (2010), 187-279.

[380] P. Scott, T. Wall, Topological methods in group theory, in Homological group theory, pp. 137-203, London Math. Soc. Lecture Note Ser., 36, Cambridge Univ. Press, Cambridge-New York, 1979.

[381] Z. Sela, Endomorphisms of hyperbolic groups. I. The Hopf property, Topology 38 (1999), no. 2, 301-321.

[382] Z. Sela, The isomorphism problem for hyperbolic groups. I, Ann. of Math. (2) 141 (1995) 217-283.

[383] A. Selberg, Discontinuous groups and harmonic analysis, 1963 Proc. Internat. Congr. Mathematicians (Stockholm, 1962), pp. 177-189 Inst. Mittag-Leffler, Djursholm.

[384] J. P. Serre, Cohomologie des groupes discrets, Prospects in mathematics, pp. 77-169. Ann. of Math. Studies, No. 70, Princeton Univ. Press, 1971.

[385] J. P. Serre, Arithmetic groups, in Homological group theory (Proc. Sympos., Durham, 1977), pp. 105-136, London Math. Soc. Lecture Note Ser., 36, Cambridge Univ. Press, Cambridge-New York, 1979.

[386] R. Sharma, T. Venkataramana, Generations for arithmetic groups. Geom. Dedicata 114 (2005), 103-146.

[387] G. Shimura, Introduction to the arithmetic theory of automorphic functions. Kano Memorial Lectures, No. 1. Publications of the Mathematical Society of 
Japan, No. 11. Iwanami Shoten, Publishers, Tokyo; Princeton University Press, Princeton, N.J., 1971. xiv+267 pp.

[388] T. Shiota, Characterization of Jacobian varieties in terms of soliton equations, Invent. Math. 83 (1986), no. 2, 333-382.

[389] T. Shiota, The KP equation and the Schottky problem, Sugaku Expositions 3 (1990), no. 2, 183-211.

[390] C. L. Siegel, Discontinuous groups, Ann. of Math. (2) 44, (1943). 674-689.

[391] C. L. Siegel, Topics in complex function theory. Vol. I. Elliptic functions and uniformization theory, Reprint of the 1969 edition. Wiley Classics Library. A Wiley-Interscience Publication. John Wiley \& Sons, Inc., New York, 1988. xii+186 pp.

[392] C. L. Siegel, Lectures on the geometry of numbers, Springer-Verlag, Berlin, 1989. $\mathrm{x}+160$ pp.

[393] C. L. Siegel, Lectures on advanced analytic number theory, Notes by S. Raghavan. Tata Institute of Fundamental Research Lectures on Mathematics, No. 23 Tata Institute of Fundamental Research, Bombay 1965 iii+331+iii pp.

[394] J. Stallings, On torsion-free groups with infinitely many ends, Ann. of Math. (2) $881968312-334$.

[395] C. Soulé, An introduction to arithmetic groups, in Frontiers in number theory, physics, and geometry, II, pp. 247-276, Springer, Berlin, 2007.

[396] D. Sullivan, Disjoint spheres, approximation by imaginary quadratic numbers, and the logarithm law for geodesics, Acta Math. 149 (1982) 215-237.

[397] D. Sullivan, The density at infinity of a discrete group of hyperbolic motions, Inst. Hautes Études Sci. Publ. Math. 50 (1979) 171-202.

[398] B. Sury, The congruence subgroup problem: an elementary approach aimed at applications, Text and Readings in Mathematics Series, Number 24, Hindustan Book Agency, 2003, xvi+301 pp.

[399] F. Taherkhani, The Kazhdan property of the mapping class group of closed surfaces and the first cohomology group of its cofinite subgroups, Experiment. Math. 9 (2000), no. 2, 261-274.

[400] K. Takeuchi, Arithmetic triangle groups, J. Math. Soc. Japan 29 (1977), no. 1, 91-106.

[401] O. Tavgen, Bounded generation of Chevalley groups over rings of algebraic $S$-integers, Math USSR Izvestiya 36 (1991) 101-128.

[402] A. Terras, Harmonic analysis and symmetric spaces and applications. II, Springer-Verlag, Berlin, 1988. xii+385 pp.

[403] A. Fathi, F. Laudenbach, V. Poénaru, Travaux de Thurston sur les surfaces, Soc. Math. France, Paris, 1979 Astérisque No. 66-67 (1991). Société Mathmatique de France, Paris, 1991. pp. 1-286.

[404] W. Thurston, Minimal stretch maps between hyperbolic surfaces, arXiv:math/9801039. 
[405] W. Thurston, On the geometry and dynamics of diffeomorphisms of surfaces, Bull. Amer. Math. Soc. (N.S.) 19 (1988), no. 2, 417-431.

[406] W. Thurston, Three-dimensional geometry and topology. Vol. 1. Edited by Silvio Levy. Princeton Mathematical Series, 35. Princeton University Press, Princeton, NJ, 1997. x+311 pp.

[407] J. Tits, Free subgroups in linear groups, J. Algebra 201972 250-270.

[408] G. van der Geer, Hilbert modular surfaces, Ergebnisse der Mathematik und ihrer Grenzgebiete (3), 16. Springer-Verlag, Berlin, 1988. x+291 pp.

[409] M. Vignéras, Arithmétique des algèbres de quaternions, Lecture Notes in Mathematics, 800. Springer, Berlin, 1980. vii+169 pp.

[410] É. Vinberg, O. Shvartsman, Discrete groups of motions of spaces of constant curvature, in Geometry, II, 139-248, Encyclopaedia Math. Sci., 29, Springer, Berlin, 1993.

[411] E. Vinberg, V. Gorbatsevich, O. Shvartsman, Discrete subgroups of Lie groups, in Lie groups and Lie algebras, II, 1-123, 217-223, Encyclopaedia Math. Sci., 21, Springer, Berlin, 2000.

[412] K. Vogtmann, Automorphisms of free groups and outer space, Geom. Dedicata 94 (2002), 1-31.

[413] K. Vogtmann, End invariants of the group of outer automorphisms of a free group, Topology 34 (1995) 533-545.

[414] K. Vogtmann, The cohomology of automorphism groups of free groups, International Congress of Mathematicians. Vol. II, 1101-1117, Eur. Math. Soc., Zürich, 2006.

[415] C. Walsh, The horoboundary and isometry group of Thurston's Lipschitz metric, arXiv:1006.2158v1.

[416] Y. Watatani, Property T of Kazhdan implies Property FA of Serre, Math. Japon. 27 (1982), no. 1, 97-103.

[417] B. Wehrfritz, Infinite linear groups. An account of the group-theoretic properties of infinite groups of matrices, Springer-Verlag, New York-Heidelberg, 1973. xiv +229 pp.

[418] H. Weyl, The theory of reduction for arithmetical equivalence, Trans. Am. Math. Soc. 48 (1940) 126-164;

[419] H. Weyl, Theory of reduction for arithmetical equivalence. II, Trans. Amer. Math. Soc. 51 (1942) 203-231.

[420] T. White, Fixed points of finite groups of free group automorphisms, Proc. Amer. Math. Soc. 118 (1993), no. 3, 681-688.

[421] W. Woess, Random walks on infinite graphs and groups, Cambridge Tracts in Mathematics, 138. Cambridge University Press, Cambridge, 2000. xii+334 pp.

[422] J. Wolf, Spaces of constant curvature. Fifth edition. Publish or Perish, Inc., Houston, TX, 1984. xviii+412 pp. 
[423] M. Wolf, The Teichmüller theory of harmonic maps, J. Differential Geom. 29 (1989) 449-479.

[424] S. Wolpert, Geodesic length functions and the Nielsen problem, J. Differential Geom. 25 (1987), no. 2, 275-296.

[425] S. Wolpert, Geometry of the Weil-Petersson completion of Teichmüller space, in Surveys in differential geometry, Vol. VIII, 357-393, Surv. Differ. Geom., VIII, Int. Press, Somerville, MA, 2003.

[426] S. Wolpert, The Weil-Petersson metric geometry, in Handbook of Teichmüller theory, Vol. II, 47-64, IRMA Lect. Math. Theor. Phys., 13, Eur. Math. Soc., Zürich, 2009.

[427] S. Wolpert, Families of Riemann Surfaces and Weil-Petersson Geometry, CBMS Regional Conference Series in Mathematics, 2010.

[428] D. Yasaki, On the existence of spines for $\mathbb{Q}$-rank 1 groups, Selecta Math. (N.S.) 12 (2006), no. 3-4, 541-564.

[429] M. Yoshida, Hypergeometric functions, my love. Modular interpretations of configuration spaces. Aspects of Mathematics, E32. Friedr. Vieweg \& Sohn, Braunschweig, 1997. xvi+292 pp.

[430] M. Yoshida, Fuchsian differential equations. With special emphasis on the Gauss-Schwarz theory. Aspects of Mathematics, E11. Friedr. Vieweg \& Sohn, Braunschweig, 1987. xiv+215 pp.

[431] S. K. Yeung, Bergman metric on Teichmüller spaces and moduli spaces of curves, in Recent progress on some problems in several complex variables and partial differential equations, pp. 203-217, Contemp. Math., 400, Amer. Math. Soc., Providence, RI, 2006.

[432] R. Zimmer, Ergodic theory and semisimple groups, Monographs in Mathematics, 81. Birkhuser Verlag, Basel, 1984. x+209 pp.

[433] S. Zucker, On the reductive Borel-Serre compactification: $L^{p}$-cohomology of arithmetic groups (for large p), Amer. J. Math. 123 (2001), no. 5, 951-984. 